U.S. DEPARTMENT OF THE INTERIOR GEOLOGICAL SURVEY

Network-Day Tape Software Users Guide

\author{
by \\ Madeleine Zirbes \\ Ray Buland
}

Open-File Report 81-666

Denver, Colorado

1981 


\title{
Network-Day Tape Software Users Guide
}

\author{
Madeleine Zirbes \\ Ray Buland
}

\section{ABSTRACT}

The Global Digital Seismograph Network (GDSN) data is now being collected into a new network-day tape format. This users guide describes the programs developed for reading network-day tapes and manipulating the data. Please keep in mind that the software described is not intended to be a general purpose analysis package. It is intended to provide adequate access to GDSN data in as machine-independent a manner as possible. Some modifications will probably have to be made to the software to use it at your site. Notes on installation are provided. 


\section{INTRODUCTION}

Software has been developed to read, extract, and manipulate data from network-day tapest. Two programs and several user callable subroutines have been developed for this purpose. The program ndtsum writes a summary of the data collected on a network-day tape. The program retrv locates selected stationcomponent-time intervals from a network-day tape and writes this information to disk files. The user callable subroutines provide a mechanism for reading and interpreting the data converted by retrv in application programs.

\section{Programs}

\subsection{Ndtsum}

Ndtsum produces a literal dump of all data logs on a networkday tape, and a list of the start and end times of each interval of data recorded for each instrument at a station. The data logs contain calibration information, station parameters, timing corrections and comments on data quality for every station included on the tape. Short-period and intermediate-period data records are written only when signals are detected, whereas long-period data are recorded continuously. Thus the list of start and end times provides a consistency check on the binary data.

\subsubsection{Ndtsum Input}

Ndtsum reads from the standard input device (FORTRAN logical unit 5) to obtain the tape drive unit number on which the networkday tape is mounted. (No prompt is given to the user.) The format for the tape drive number is I1.

\subsubsection{Ndtsum Output}

All output from ndtsum is directed to the standard output device (FORTRAN logical unit 6). If an error occurs, a message is printed informing the user where the problem was, before exiting the program.

Listed below is an example of the output produced by ndtsum. Although this is not a complete listing, it does include an example of all the different types of logs written on a network-day tape.

+ Hoffman, John P., 1980, The Global Digital Seismograph Network-Day Tape, U.S. Ceological Survey Open-File Report 80-289, 37 p. 
GLOBAL DIGITAL SEISMOGRAPH NETWORK-DAY TAPE LOG

TAPE FILE: 1

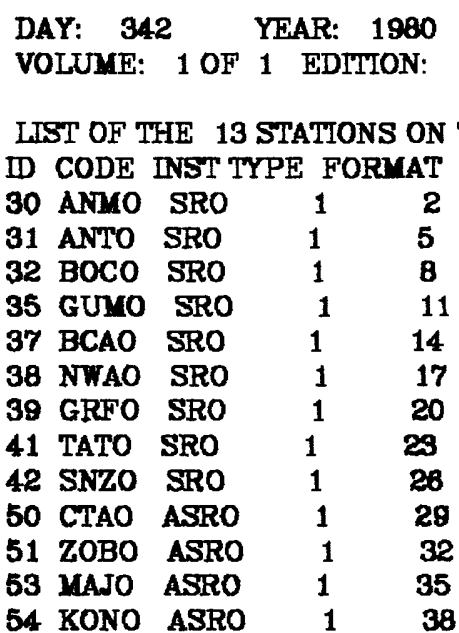

STATION LOG:ALBUQUERQUE, NEW MEXICO TAPE FILE: 2

DAY: 342 YEAR: 1980 DATE: TDEC80 NUMBER OF STATIONS ON THIS LOG: 1

STATION LIST: ID CODE INST TYPE LATTTUDE LONGITUDE ELEVATION(M) SO ANMO SRO 34.9462N 106.4568W 1740.0M

DATA FHES: 2

FILE NO ID DESCRIPTION

3 SO LONG PERIOD Z,N,E

4 SO SHORT PERIOD Z

TINE CORRECTIONS: 1

YEAR DAY TDE CORRECTTON(SEC)

$19803420000-00.001 /-$

DATA OUTAGES OF MORE THAN ONE HOUR: 0

FROM: DAY TTME TO: DAY TME

CONMENTS:

NONE.

DATA LOG:FOR 3 CHANNEIS LONG PERIOD Z,N,E RBCORDTNG MODE: CONTINUOUS, MULTIPLEXED

TAPE FIE: 3

SAYPLE RATE: 1.0/SEC SAMPLE INTERVAL: 1.000 SEC

CHANNEL SEQUENCE: $1=$ VERTICAI, $2=$ NORTH, $3=$ EAST

CALIBRATION DATA

CHANNEL YEAR DAY TIE AMPLITUDE AVE CAL VALUE FREQUENCY (COUNTS/MTCROI ETER) (COUNTS/MTCRONETER) (HZ)

$1 \quad 1880342000 \quad 4.5409 \mathrm{E}+03$

$1880343000 \quad 4.4482 \mathrm{E}+03$

$1980342000 \quad 4.8276 \mathrm{~F}+03$

$1980343000 \quad 4.8682 E+03$

$1880342000 \quad 5.5281 \mathrm{E}+03$

4. $4745 E+03$

$4.0095 \mathrm{E}-02$

$4.4745 \mathrm{E}+03$

4.845.25 +03

$3.9996 \mathrm{E}-02$

$4.8452 E+03 \quad 4.00065-02$

$5.5055 \mathrm{E}+03 \quad 4.00922-02$

$1880 \$ 43000 \quad 5.4907 \mathrm{E}+03 \quad 5.5055 \mathrm{E}+03 \quad 4.0013 \mathrm{E}-02$ 
THE COMPIEX TRANSFER FUNCTTONS T ARE CALCULATED BY:

$T(S)=A 0^{* D S} *(S-Z 01) *(S-Z 02) * \ldots *(S-Z M) /((S-P 01) *(S-P 02) * \ldots(S-P M))$

WHERE: $\mathbf{S}=\mathrm{J} * \mathbf{W}, \mathbf{W}=A N G U L A R$ FREQUENCY, AO IS SCAIAAR, M IS THE NUMBER OF COMPLEX ZEROS, N IS THE NUMBER OF COMPIES POLES, THE Z'S AND THE P'S ARE THE COMPLEX ZEROES AND POLFS OF THE SYSTEM, AND DS IS THE APPROPRIATE DIGITAL SENSITTVTYY (COUNTS/MICROMETER) IN THE TABLE ABOVE

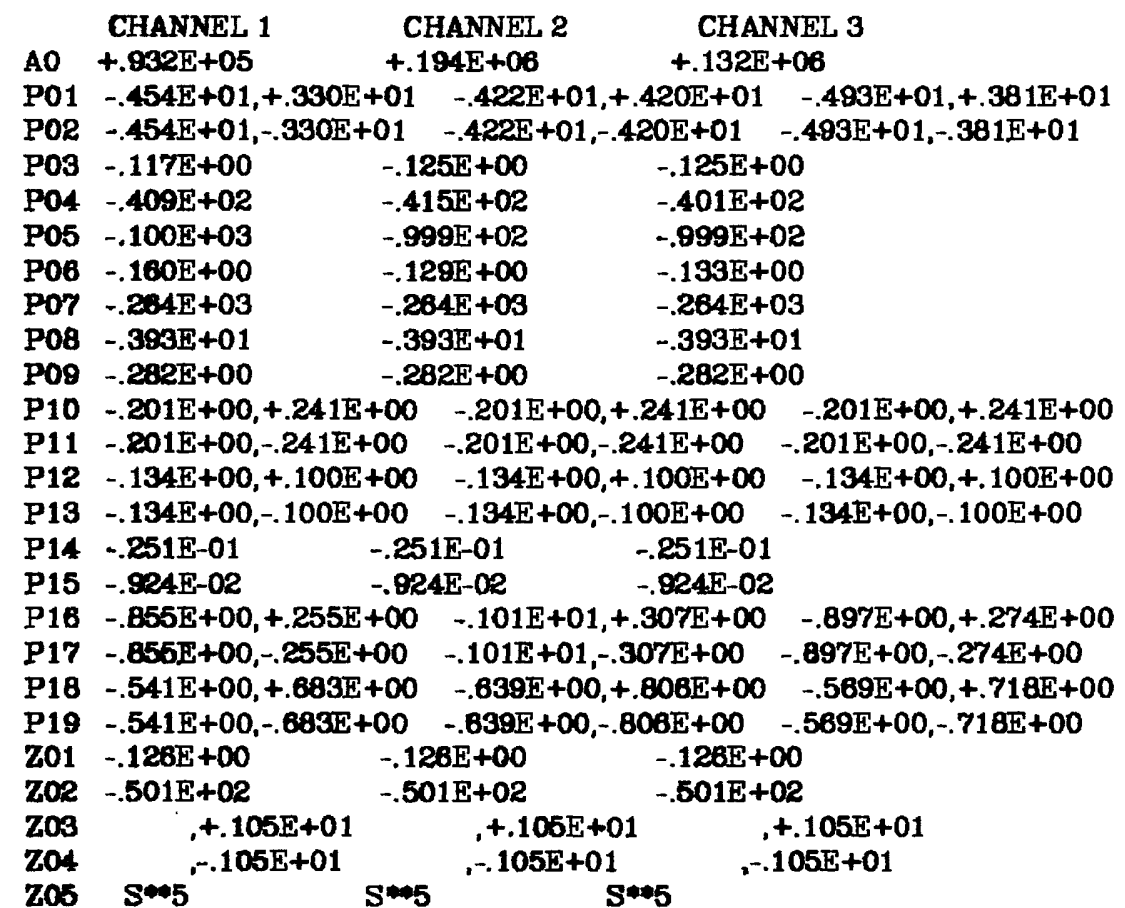

RETATTVE RESPONSE TO EARTH DISPIACEYENT

CHANNEL 1

CHANNEL 2

MEASURED-JUL, 1979
MEASURED-JUL, 1879

PERIOD AMPITIUDE PHASE PERIOD AMPLIIUDE PHASE PERIOD AMPLITUDE PHASE

(SEC) (DEG) (SEC) (DEG) (SEC) (DEG)

$1022+.165 \mathrm{E}-043021011+.195 \mathrm{E}-043921006+.199 \mathrm{E}-04391$

$516+.342 E-03351514+.383 E-02 \quad 352502+.412 E-03 \quad 349$

$268+.509 \mathrm{E}-0 \mathrm{Q} 296258+.554 \mathrm{E}-02297251+.599 \mathrm{E}-02293$

$98.0+.110 \mathrm{E}+00 \quad 190 \quad 99.2+.118 \mathrm{E}+00 \quad 188 \quad 100.4+.112 \mathrm{E}+00188$

$78.4+.206 \mathrm{E}+\infty \quad 167 \quad 79.6+.214 \mathrm{E}+00 \quad 167 \quad 79.9+.206 \mathrm{E}+00167$

$58.6+.405 \mathrm{E}+00 \quad 122 \quad 59.6+.429 \mathrm{E}+00 \quad 122 \quad 59.8+.415 \mathrm{E}+00 \quad 122$

$50.1+.573 \mathrm{E}+00 \quad 90 \quad 50.0+.598 \mathrm{E}+00 \quad 80 \quad 48.2+.635 \mathrm{E}+00 \quad 85$

$39.7+.623 \mathrm{E}+00 \quad 42 \quad 38.9+.632 \mathrm{E}+00 \quad 45 \quad 40.0+.634 \mathrm{E}+00 \quad 42$

$30.1+.102 \mathrm{E}+01-24 \quad 30.1+.102 \mathrm{E}+01-20 \quad 30.1+.103 \mathrm{E}+01-25$

$25.0+.100 \mathrm{E}+01-72 \quad 24.9+.100 \mathrm{E}+01-68 \quad 25.0+.100 \mathrm{E}+01-73$

$20.0+.601 \mathrm{E}+00-132 \quad 20.0+.608 \mathrm{E}+00-124 \quad 20.0+.807 \mathrm{E}+00-131$

$14.5+.392 \mathrm{E}+00-21 \theta \quad 14.4+.395 \mathrm{E}+00-207 \quad 15.0+.436 \mathrm{E}+00-209$

$8.0+.883 E-01-319 \quad 9.9+.102 E+\infty 0-298 \quad 10.0+.100 E+00-312$

$7.8+.259 \mathrm{E}-01-378 \quad 7.8+.286 \mathrm{E}-01-354 \quad 8.0+.284 \mathrm{E}-01-364$

START TTEE

YEAR MONTH DAY TIME

1880127 0: $0: 0.580$.
END TIME

YEAR YONTH DAY TTME 
DATA IOG:FOR 1 CHANNEL SHORT PERIOD $\mathrm{Z}$ TAPE FIIE: 4 RECORDING MODE: TRIGGERED

FORMAT TYPE: 1

SAMPIT RATE: 20.0/SEC SAMPIE INTERVAL: 0.050 SEC

CHANNEL SEQUENCE: 1 =VERTICAL

CAIIBRATION DATA

CHANNEL YEAR DAY TTME AMPIITUDE AVE CAL VALUE FREQUENCY

\begin{tabular}{llll} 
& \multicolumn{4}{c}{ (COUNTS/MICROMETER) } & (COUNTS/MICROMETER) \\
1 & 1880342 & $1.9815 \mathrm{E}+06$ & $1.0004 \mathrm{E}+00$ \\
1 & 1830343 & $1.9815 \mathrm{E}+06$ & $1.0004 \mathrm{E}+00$
\end{tabular}

THE COMPLEX TRANSFER FUNCTIONS T ARE CALCUIATED BY:

$T(S)=A 0^{* D S} *(S-Z 01) *(S-Z 0 Z) * \ldots *(S-Z M) /((S-P 01) *(S-P 0 Z) * \ldots(S-P M))$

WHBRE: $3=J \oplus \%, W=A N G U L A R$ FREQUENCY, AO IS SCAIAR, M IS THE NUMBER OF

COMPIEX ZEROS, N IS THE NUMBER OF COMPLES POLES, THE Z'S AND THE P'S

ARE THE COMPLEX ZEROES AND POLES OF THE SYSTEM, AND DS IS THE APPROPRIATE

DIGITAL SENSITIVTTY (COUNTS/MICROMETER) IN THE TABLE ABOVE

CHANNEL 1

ANMO SP TRANSFER FUNCTION

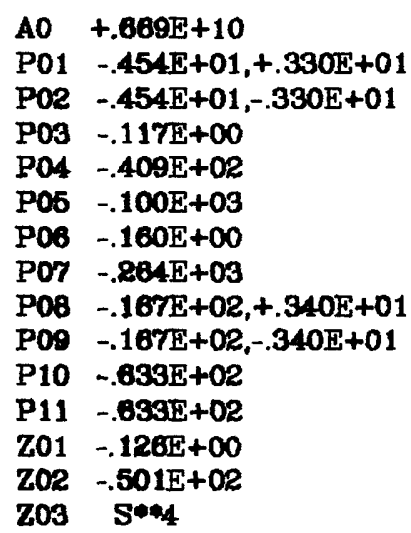

RELATIVE RESPONSE TO EARTH DISPLACEMENT

CHANNEL 1

MEASURED-JUL1979

PERIOD AMPLIUUDE PHASE

(SEC) (DEG)

$10.000+.207 \mathrm{E}-02285$

$5.000+.164 \mathrm{E}-01244$

$2.500+.120 \mathrm{E}+00207$

$2.000+.221 E+00191$

$1.670+.951 \mathrm{E}+00174$

$1.250+.67225+00143$

$1.000+.100 \mathrm{E}+01116$

$.883+.129 \mathrm{E}+0191$

$.687+.161 \mathrm{E}+0163$

$.500+.1898+0125$ 
$.393+.194 \mathrm{E}+01-30$

$.250+.168 E+01-68$

$.200+.1325+01-99$

$.143+.843 E+00-145$

\begin{tabular}{|c|c|c|c|c|c|c|c|}
\hline \multicolumn{4}{|c|}{ START TIME } & \multicolumn{3}{|c|}{ END TDE } & TTME \\
\hline 1980 & 12 & 7 & $0: 27: 55.580$ & 1980 & 12 & 7 & $0: 28: 44.530$ \\
\hline 980 & 12 & 7 & $1: 50: 14.580$ & 1980 & 12 & 7 & 1:59:30.530. \\
\hline 1980 & 12 & 7 & 2:46:30.580. & 1980 & 12 & 7 & 2:48: 8.530. \\
\hline 1880 & 12 & 7 & $2: 59: 38.580$ & 1980 & 12 & 7 & 3: 0:27.530. \\
\hline 980 & 12 & 7 & 4: 7:57.580. & 1980 & 12 & 7 & 4: 8:46.530 \\
\hline 980 & 12 & 7 & $6: 15: 16.580$ & 1980 & 12 & 7 & 5.530. \\
\hline 1980 & 12 & 7 & 6:24: 5.580 & 1980 & 12 & 7 & 4.530. \\
\hline 1880 & 12 & 7 & $8: 48: 54.580$ & 1980 & 12 & 7 & 3.530 . \\
\hline 1980 & 12 & 7 & 9: 3:13.580. & 1980 & 12 & 7 & 9: $5: 40.530$ \\
\hline 1980 & 12 & 7 & $9: 9: 10.580$ & 1980 & 12 & 7 & 9:11:37.530. \\
\hline 1980 & 12 & 7 & 9:45:37.580. & 1980 & 12 & 7 & $9: 47$ \\
\hline 980 & 12 & 7 & 10:42:45.580. & 1980 & 12 & 7 & \\
\hline 1880 & 12 & 7 & $11: 16: 4.580$ & 1980 & 12 & 7 & $11: 16$ \\
\hline 1980 & 12 & 7 & 13:32:53.580. & 1980 & 12 & 7 & $13: 33: 42$ \\
\hline 1980 & 12 & 7 & $14: 47: 12580$ & 1880 & 12 & 7 & $14: 48: 1.530$ \\
\hline 1980 & 12 & 7 & 15: 8: 1.580 & 1980 & 12 & 7 & $15: 10: 28.53$ \\
\hline 1880 & 12 & 7 & 15:30:58.580. & 1880 & 12 & 7 & $15: 3$ \\
\hline 1980 & 12 & 7 & 16:10:47.580. & 1980 & 12 & 7 & 16: 1 \\
\hline 1980 & 12 & 7 & 17:48: 6.580 & 1980 & 12 & 7 & $17: 54: 49.5$ \\
\hline 1980 & 12 & 7 & 19: 3:19.580. & 1880 & 12 & 7 & 19: 4:57.530. \\
\hline 1980 & 12 & 7 & 19:27:27.580. & 1880 & 12 & 7 & $19: 28: 16.530$ \\
\hline 1880 & 12 & 7 & 18:30:16.580. & 1880 & 12 & 7 & 19:91: 5 \\
\hline 1980 & 12 & 7 & $20: 35: 35.580$ & 1980 & 12 & 7 & $38: 24$ \\
\hline 1980 & 12 & 7 & 20:46:54.580. & 1980 & 12 & 7 & $80: 47: 49.5$ \\
\hline 1980 & 12 & 7 & 21: 3:43.580. & 1980 & 12 & 7 & 21: 4:32.53 \\
\hline 1880 & 12 & 7 & 22:10: 2.580. & 1980 & 12 & 7 & $22: 11: 40.530$. \\
\hline 1080 & 12 & 7 & $22: 57: 40.580$ & 1880 & 12 & 7 & 22:59:18.5 \\
\hline 980 & 12 & 8 & 0: 8:18.580. & 1880 & 12 & 8 & 56.53 \\
\hline & 12 & B & 0:30:58.580. & 1880 & 12 & 8 & $0: 31: 45.530$ \\
\hline 1880 & 12 & 8 & 1:34:45.580. & 1980 & 12 & $\boldsymbol{\theta}$ & 1:35:34.530. \\
\hline 1980 & 12 & a & 4:35: 4.580 & 1880 & 12 & 8 & $4: 35: 53.58$ \\
\hline
\end{tabular}

Note the list of start and end times in the above listing. This information is not contained on a $\log$, but is produced by ndtsum to provide a consistency check on the binary data.

\subsection{Retrv}

Retrv locates selected information from a network-day tape and writes this information into binary output files.

\subsubsection{Retrv Input}

Retrv reads from the standard input device (FORTRAN logical unit 5) to obtain the tape drive unit number on which the networkday tape is mounted. The format for the tape drive number is I1. Retrv then continues to read requests from the standard input to determine what information is to be extracted from the network- 
day tape. (No prompt is given to the user to obtain either the tape drive number or the requests.) The requests define station, instrument, and time interval information that the user wants extracted. Each request line specifies the following information:

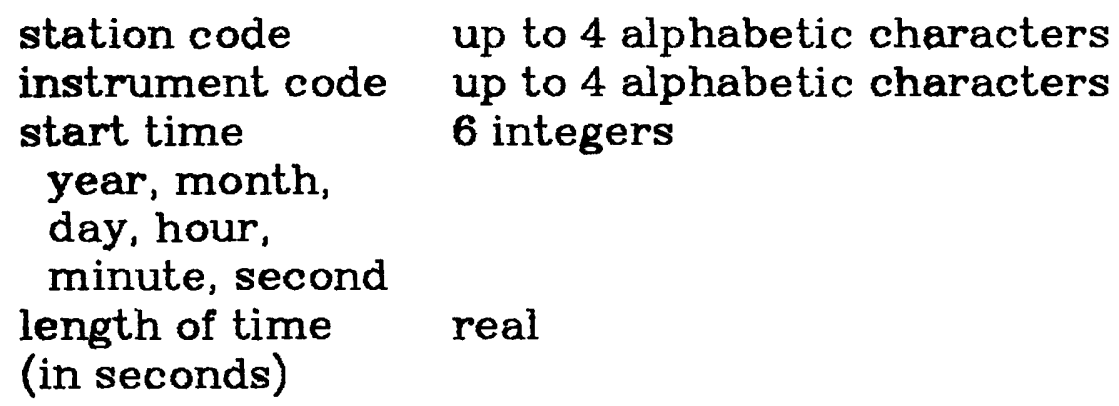

The station code is the four-letter character string (upper case) of the station desired. It can optionally be the character string "ALL", to retrieve the specified instrument and time interval information for all the stations recorded on the tape.

The instrument code is a four-letter character string (upper case) indicating the instrument and orientation of data desired. The first character of the instrument code can be:
S for short-period instrument,
L for long-period instrument, or
I for intermediate-period instrument.

The second, third, and fourth characters of the instrument code specify the orientation of the data wanted and can be:

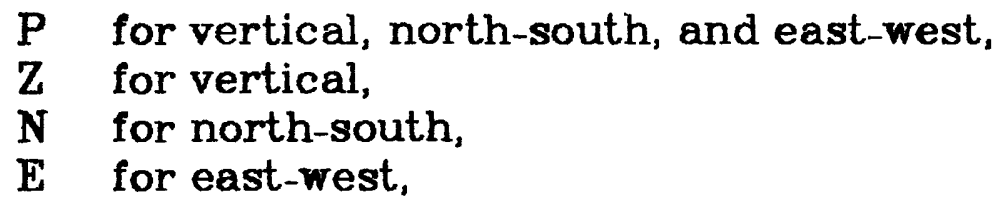

or any combination of the characters $\mathrm{Z}, \mathrm{N}, \mathrm{E}$ if more than one orientation of data is desired. The instrument code can also be the character string "ALL", retrieving each orientation of data for each instrument recorded.

The input is free format with the fields delimited by blanks or commas. Each request cannot be longer than 80 characters. To indicate that there are no more requests, the last request line must be the character string "STOP".

For example, a sample request line might be 
This request line is asking that data from Albuquerque, New Mexico, be extracted from the long-period instrument, vertical, northsouth, and east-west orientations, starting at 16:33:44 on 25 May, 1980 , and ending 3600 seconds later.

The request line

$$
\text { ALL,ALL, 1980,5,25, 19,44,51, } 1800
$$

would retrieve all short-period, long-period, and intermediateperiod data from all the stations recorded on the tape starting at 19:44:51 on 25 May, 1980, for a length of 1800 seconds.

\subsubsection{Retrv Output}

Retrv opens seven output files, one for each type of data that could be retrieved. The output file names and their corresponding data types are:

$\begin{array}{ll}\text { RETRV.SPZ } & \text { short-period vertical data } \\ \text { RETRV.LPZ } & \text { long-period vertical data } \\ \text { RETRV.LPN } & \text { long-period north-south data } \\ \text { RETRV.LPE } & \text { long-period east-west data } \\ \text { RETRV.IPZ } & \text { intermediate-period vertical data } \\ \text { RETRV.IPN } & \text { intermediate-period north-south data } \\ \text { RETRV.IPE } & \text { intermediate-period east-west data }\end{array}$

These binary output files contain two types of records: header records and data records. Header records contain station information. Data records contain the actual data extracted, preceded by the number of data values contained in the data record.

Header and data records are written to the appropriate output files as each request is satisfied. Header records are written to the output files first, followed by one or more data records. This means that an output file may contain several header records, each followed by data records. To indicate that a data record is the last data record for a particular request, a null data record is written having one data value that is equal to $10,000,000,000.0$. To indicate that there are no more records in the output file, a final null header record is written just before the file is closed. A null header record has the station code and instrument code set to "END", the number of poles and zeroes set to one, and all other values set to zero.

A schematic representation of the format of these binary files is: 
INTEGER SMN

C SECONDS

INTEGER SSEC

C MIIJISECONDS

REAL SMS

C

C END TIME OF CONTINUOUS DATA

C

C YEAR

INTEGER LYR

C DAY OF YEAR

INTEGER IDY

C HOUR

INTEGER LHR

C MINUTE

INTEGER IMN

C SECONDS

INTEGER ISEC

C MUIUSECONDS

REAL IMS

C BINARY DATA

INTEGER IDATA(1000)

C NUMBER OF SAMPLES OF LAST DATA READ INTEGER INSAM

C NUMBER OF CHANNEIS OF IAST DATA READ INIEGER LNCHAN

C NEXT EXPECTED TIME FOR DATA INTEGER NYR, NDY, NHR, NMN, NSEC

C CURRENT TTME OF DATA INIEGER TYR, TDY, THR, TMN, TSEC REAL TMS

C LOOP INDEX

INTEGER I

INTEGER IBUFF(80)

C FUNCTION

INTEGER MAGRD

C NUMBER OF FRAMES READ

INTEGER NR

INTEGER KUS, KUT

C MIIJISECONDS

REAL FMS

REAL NMS

C SAMPLING RATE OF LAST DATA READ

REAL LRATE

C NUMBER OF FRAMES OF DATA TO READ

INTEGER NWORDS

C

COMMON /HEADER/ ID, INSTR, YEAR, DOFY, HOUR, MTN, SEC, MSS, NFORM,

C

1 NCHAN, NSAMP. RATE

C

COMMON /START/ SYR, SDY, SHR, SMN, SSEC, SMS

C

COMMON /END/ LYR, LDY, LHR, IMN, ISEC, LMS

DATA NWORDS $/ 1000 /$

C

DATA KUS, KUT /'S", "T"/

C

NETWORK DAY TAPE FORMAT

C EOF EOF

C EOF EOF

c 11 
data record

data record

null data record
header record
data record

null data record

null header record

When retrv has satisfied a request, a statement summarizing the data that was extracted is written to the standard output device. This summary consists of the station code, instrument code, year, month, day, start time of the data, and length of the data extracted, in seconds.

For example, if data was found to satisfy request line (A), the output lines describing what in fact was extracted might be:

$\begin{array}{llllll}\text { ANMO LPZ } & 1980 & 5 & 25 & 16: 33: 44.53 & 3600.000 \\ \text { ANMO IPN } & 1980 & 5 & 25 & 16: 33: 44.53 & 3600.000 \\ \text { ANMO LPE } & 1980 & 5 & 25 & 16: 33: 44.53 & 3600.000\end{array}$

If data was found satisfying request line (B), the output lines describing what was retrieved might be:

\begin{tabular}{|c|c|c|c|c|c|}
\hline ANMO LPZ & 1980 & 5 & 25 & $19: 44: 51.53$ & 1800.000 \\
\hline ANMO IPN & 1980 & 5 & 25 & $19: 44: 51.53$ & 1800.000 \\
\hline ANMO IPE & 1880 & 5 & 25 & $19: 44: 51.53$ & 1800.000 \\
\hline ANMO SPZ & 1980 & 5 & 25 & $19: 44: 51.53$ & 1800.000 \\
\hline ANTO LPZ & 1880 & 5 & 25 & $19: 44: 51.91$ & 00. \\
\hline ANTO IRN & 1880 & 5 & 25 & $10: 44: 51.91$ & 1800.0 \\
\hline ANTO IPE & 1980 & 5 & 25 & $19: 44: 51.91$ & 1800.000 \\
\hline ANTO SPZ & 1980 & 5 & 25 & 20:11: 3.91 & 88.000 \\
\hline BOCO IPZ & 1980 & 5 & 25 & $19: 44: 51.91$ & 1600.000 \\
\hline BOCO IPN & 1980 & 5 & 25 & $19: 44: 51.91$ & 1600.000 \\
\hline BOCO IPE & 1980 & 5 & 25 & $19: 44: 51.91$ & 1800.000 \\
\hline BOCO SPZ & 1980 & 5 & 25 & 19:53:43.91 & 1800.000 \\
\hline CHTO LPZ & 1960 & 5 & 25 & $19: 44: 51.93$ & 1800.000 \\
\hline CHTO LPN & 1980 & 5 & 25 & $19: 44: 51.93$ & 1800.000 \\
\hline CHTO IPE & 1880 & 5 & 25 & $19: 44: 51.83$ & 1800.000 \\
\hline CHTO SPZ & 1860 & 5 & 25 & 20: 4: 9.93 & 147.000 \\
\hline CHTO SPZ & 1980 & 5 & 25 & 20: 8: $\quad .93$ & 49.000 \\
\hline GUMO IPZ & 1980 & 5 & 25 & $18: 44: 51.79$ & 1800.000 \\
\hline cring & 1980 & 5 & 25 & $19: 44: 51.79$ & 1800.000 \\
\hline GU & 1980 & 5 & 25 & $19: 44: 51.79$ & 1800.000 \\
\hline & 1880 & 5 & 25 & $18: 44: 51.39$ & 1800.00 \\
\hline
\end{tabular}




\begin{tabular}{|c|c|c|c|c|c|}
\hline BCAO LPN & 1980 & 5 & 25 & 19:44:51.39 & 1800.000 \\
\hline BCAO IPE & 1980 & 5 & 25 & $19: 44: 51.39$ & 1800.000 \\
\hline BCAO SPZ & 1980 & 5 & 25 & 20: 8:11.39 & 294.000 \\
\hline NWAO I.PZ & 1880 & 5 & 25 & $19: 44: 51.92$ & 1800.000 \\
\hline NWAO LPN & 1980 & 5 & 25 & $19: 44: 51.92$ & 1800.00 \\
\hline NTAO LPE & 1980 & 5 & 25 & $19: 44: 51.92$ & 1800.0 \\
\hline GRFO IPZ & 1980 & 5 & 25 & $19: 44: 51.93$ & 1800.00 \\
\hline GRFO I.PN & 1980 & 5 & 25 & $19: 44: 51.93$ & 1800.00 \\
\hline GRFO LPE & 1880 & 5 & 25 & $19: 44: 51.93$ & 1800.00 \\
\hline GRFO SPZ & 1880 & 5 & 25 & $19: 58: 50.93$ & \\
\hline TATO IPZ & 1980 & 5 & 25 & $19: 44: 51.91$ & 1800.0 \\
\hline TATO IPN & 1980 & 5 & 25 & $18: 44: 51.91$ & 1600.00 \\
\hline TATO LPE & 1980 & 5 & 25 & $19: 44: 51.91$ & 1800.00 \\
\hline SNZO I.PZ & 1980 & 5 & 25 & $19: 44: 51.83$ & 1800.0 \\
\hline SNZO LPN & 1880 & 5 & 25 & $18: 44: 51.93$ & 1600.00 \\
\hline SWZO IRPE & 1980 & 5 & 25 & $19: 44: 51.93$ & 1800.00 \\
\hline CTAO I.PZ & 1980 & 5 & 25 & $19: 44: 51.93$ & 1800.00 \\
\hline CTAO I.PN & 1980 & 5 & 25 & 19:44:51.93 & 1600.00 \\
\hline CTAO LPE & 1980 & 5 & 25 & 19:44:51.93 & 1800.00 \\
\hline ZOBO LPZ & 1980 & 5 & 25 & 19:44:51.84 & 1800.0 \\
\hline ZOBO LPN & 1980 & 5 & 25 & $19: 44: 51.94$ & 1800.0 \\
\hline ZOBO LPE & 1880 & 5 & 25 & $19: 44: 51.94$ & 1800.00 \\
\hline ZOBO SPZ & 1880 & 5 & 25 & 19:55:32.94 & 588.0 \\
\hline KAAO IAPZ & 1980 & 5 & 25 & $19: 44: 51.61$ & 1800.00 \\
\hline KAAO LPN & 1980 & 5 & 25 & 19:44:51.61 & 1800.0 \\
\hline KAAO IAPE & 1880 & 5 & 25 & $19: 44: 51.61$ & 1600.00 \\
\hline MAJO LPZ & 1980 & 5 & 25 & 19:44:51.20 & 1600.0 \\
\hline MAJO LPN & 1980 & 5 & 25 & $19: 44: 51.20$ & 1800.0 \\
\hline MANO LPE & 1980 & 5 & 25 & 19:44:51.20 & 1800.00 \\
\hline MAJO SPZ & 1980 & 5 & 25 & 19:58:30.20 & 294.00 \\
\hline KONO LPZ & 1880 & 5 & 25 & $19: 44: 51.37$ & 1800.0 \\
\hline KONO LPN & 1880 & 5 & 25 & $18: 44: 51.37$ & 1800.0 \\
\hline KONO LPE & 1880 & 5 & 25 & $19: 44: 51.37$ & 1800.0 \\
\hline KONO SPZ & 1880 & 5 & 25 & 19:58: 3.37 & 245.0 \\
\hline
\end{tabular}

The user can specify the list of stations from which he desires information in any order. But if he has requested more than one time interval for a particular station and component, these requests must be in increasing time sequence. Because retrv does not backspace the tape, requests for overlapping data are not handled.

For example, the request lines

ANTO, IP, 1980, 5, 25, 17, 0, 0, 180

ANTO, SP, 1860, 5, 25, 18, 16, 0, 450

ANTO, IP, 1980, 5, 25, 19, 5, 0,780

ANTO, IP, 1980, 5, 25, 19, 13, 0, 1020

STOP

would produce the output:

$\begin{array}{lllllll}\text { ANTO } & \text { IPZ } & 1980 & \mathbf{5} & 25 & 17: 0: .91 & \mathbf{1 8 0 . 0 0 0} \\ \text { ANTO } & \text { IPN } & 1980 & \mathbf{5} & 25 & 17: 0: .91 & \mathbf{1 8 0 . 0 0 0} \\ \text { ANTO } & \text { IPE } & 1980 & \mathbf{5} & 25 & 17: 0: .91 & \mathbf{1 6 0 . 0 0 0} \\ \text { ANTO } & \text { IPZ } & 1980 & \mathbf{5} & 25 & 19: 5: .91 & \mathbf{7 8 0 . 0 0 0} \\ \text { ANTO } & \text { IPN } & 1980 & \mathbf{5} & 25 & 19: 5: .91 & \mathbf{7 8 0 . 0 0 0} \\ \text { ANTO } & \text { LPE } & 1980 & \mathbf{5} & 25 & 19: 5: .91 & \mathbf{7 8 0 . 0 0 0} \\ \text { ANTO } & \text { IPZ } & 1890 & \mathbf{5} & 25 & 19: 15: .91 & 1020.000 \\ \text { ANTO } & \text { IPN } & 1980 & \mathbf{5} & 25 & 19: 15: .91 & 1020.000 \\ \text { ANTO } & \text { IPE } & 1980 & \mathbf{5} & 25 & 19: 15: .91 & 1020.000 \\ \text { ANTO } & \text { SPZ } & 1880 & \mathbf{5} & 25 & 18: 16: .91 & 123.000\end{array}$


Note that although the user's fourth request is for data beginning at 19:13:00, his previous request asks for data to be extracted at 19:05:00 for a length of 13 minutes, leaving the tape positioned after the record containing the start time of 19:13:00. Retrv extracts data as close to the time requested as possible extending for the requested number of seconds. One would think that the result of the fourth request would begin at 19:18:00. However, relry will overlap the third and fourth requests by part of one buffer (the last physical tape record used by the third request is still in memory when the fourth request is initiated). The fourth request actually overlaps the third by three minutes in this case.

Each time a request is satisfied retru checks the list of stations recorded on the tape against the list of stations the user has requested. If there are requests for station data recorded later on the tape, retrv continues reading the tape searching for data to salisfy lhose requesls. If nol, retrv stops. I'his means that only as much of the tape will be read as is necessary.

Mosl compulers do one of lwo lhings when a pre-exisling file is opened for output: the data in the file is overwritten, or new date is appended to the end of the file. In either case, the output files produced as a result of executing retru should be moved elsewhere before running retrv again, to insure against loss. Because retrv has its own convention for end-of-file (the null header record), even if data is appended, the input routines provided will not read past the end of the pre-exisling dala.

\subsubsection{Hetrr Files}

Tile

RETRV.SPZ

RETTRV.LPZ

RETRV.LPN

RFTTRV.T.PF:

RE"l'RV.IF'L

RETRV.IPN

RETRV.IPE

'standard-input'

'standard-output'
Logical Unit Number

1

2

3

4

7

8

9

5

6
Input/Output

Output

Output

Output

Output.

Output.

Output

Oulpul

Input

Output 


\title{
2. Routines
}

\subsection{Iasnlu, Initf, Irdhd, Irddat}

Iasniu, inith, irdhd, and irddal operale on the binary files produced by retrv. Irdhd reads header records. Irddat reads data records. Initfl initializes flags used by irdhd and irddat. Iasnlu associates a logical unit number with a data array index.

The syntax of these routines is:

\author{
CALL INITFL \\ INTEGER IERR, IASNLU, LUN, INDX \\ TFRR = IASNTIU(TIUN, INDX) \\ INTEGER IERR, IRDHD, INDX \\ IERR $=$ IRDHD $($ INDX) \\ INTEGER IERR, IRDDAT, INDX \\ REAL VALU \\ TF.RR = IRDDAT(INDX, VAT,UJ)
}

Irdhd reads the next header record from the logical unit asso ciated with indx. The header record information is stored in common block /head/. This function returns 1 upon a successful read, 0 on end of file, and -1 . for an error. Irdhd will always try to read the next header record, even though intermediate data records may have to be skipped.

On each call, irddat sequentially accesses the next data point of a dala record, returning il in valu. This funclion relurns 1 upon a successful read, 0 on end of data or end of file, and -1 if the next record is not a data record.

Initialization of flags used by irdhd and irddat is done by calling the subroutine initfl. The flags are stored in common block /flags/. Initf must be called before any reference to irdhd or irddat, to insure reading the header and data records successfully.

The function iasnlu associates a logical unit number with an index to the head and data arrays. Iasnlu returns 1 if an index was assigned, -1 if no more buffer space is available. 'The variable ind $x$ is an input to the functions irdhd and irddat.

These routines are designed to read up to three files simultaneously. Nole thal all elemenls in common/head/ have an ouler dimension of three. Information associated with a given logical unit number is buffered in space pointed to by indx. See program sample below for an example. 


\title{
2.2. Resp
}

The function resp returns the complex forward transfer function of the instrument system, at angular frequency omega.

The syntax is:

\author{
COMPLEX RESP, T \\ INTEGER IFL, INDX \\ REAL OMEGA \\ T = RESP(INDX, OMEGA, IFL)
}

The transfer function is the Fourier transform of the system impulse response using the Fourier convention $\int f(t) e^{-i \omega t} d t$. The transfer function describes how the instrument modifies ground motion as follows:

where

$$
U(\omega)=H(\omega) D(\omega)
$$

$D$ is the Fourier transform of the ground displacement,

$\mathrm{H}$ is the Fourier transform of the transfer function,

$U$ is the Fourier transform of the recorded signal, and

$\omega$ is the angular frequency in radians per sec.

The user specifies the desired output units by setting ifl appropriately. If ifl equals 1 , resp returns $H(\omega)$ at $\omega$ in units of counts/micron, assuming the input is ground displacement. If ifl equals 2 , the units are in counts/micron/sec (assuming the input is ground velocity); and if iff equals 3 , the units are in counts/micron/sec 2 (assuming the input is ground acceleration).

The variable ind $x$ is set in a reference to the function iasnlu, to indicate which array index is to be referenced.

\subsection{Common Blocks}

COMMON BLOCK HEAD

C STATION CODE

INTEGER CODE $(4,3)$

C INSTRUMENT CODE

INTEGER CHN $(4,3)$

C STATION LATITUDE

REAL LAT(3)

C STATION LONGITUDE

REAL LON(3)

C STATION ELEVATION

REAL ELEV(3)

C START TIME OF DATA

INTEGER SYEAR(3) 
INTEGER SDOFY(3)

INTEGER SHOUR(3)

INTEGER SMIN(3)

REAL SSECS(3)

C SAMPLE RATE

REAL RATE(3)

C NORMALIZATION CONSTANT

REAL AO(3)

C NUMBER OF POLES

INTEGER NP(3)

C POLES

COMPLEX POLES(20,3)

C NUMBER OF ZEROES

INTEGER NZ(3)

C ZEROES

COMPLEX ZEROES $(10,3)$

COMMON /HEAD/ CODE, CHN, LAT, LON, ELEV,

1 SYEAR, SDOFY, SHOUR, SMIN, SSECS,

1 RATE, AO, NP, POLES, NZ, ZEROES

COMMON BLOCK DATA

C NUMBER OF DATA POINTS IN BUFF

REAL PTS(3)

C DATA BUFFER

REAL BUFF $(500,3)$

COMMON /DATA/ PTS, BUFF

COMMON BLOCK FLAGS

C HEADER RECORD FLAG

INTEGER HFLG(3)

C DATA RECORD FLAG

INTEGER DFLG(3)

C NUMBER OF LOGICAL UNIT NUMBERS ASSIGNED

INTEGER NLUN

C LOGICAL UNIT NUMBERS ASSOCIATED WITH DATA INDEXES INTEGER LLUN(20)

C ASSOCIATES AN INDEX WITH A LOGICAL UNIT NUMBER INTEGER ILUN(3)

C INDEX OF NEXT DATA VALUE TO BE RETURNED

INTEGER NPTS(3)

COMMON /FLAGS / HFLG, DFLG, NLUN, ILUN, ILUN, NPTS 


\section{Installation}

\subsection{Ndtsum}

The program ndtsum produces a literal dump of a network-day tape, with a list of start and end times of the continuous data. The main program, ndtsum.fortran, reads the tape drive unit number from the standard input, and opens the tape drive for reading. It then calls a subroutine that does the work of producing the summary, and finally closes the tape drive. Ndtsum assumes that logical unit number 5 is already open for formatted input, and that logical unit number 6 is open for formatted output.

\subsection{Retrv}

The program retrv locates selected information from a network-day tape and writes this information into binary output files. The main program, retrv.fortran, reads the tape drive unit number and requests input from the standard input device. A subroutine is called to initialize the program variables. Retrv assumes that logical unit 5 is already open for formatted input.

All output files are then opened for unformatted, sequential writing. The tape drive is opened for reading. Retrv assumes that logical unit 6 is already open for formatted output. Control then passes to the subroutine that reads the tape, searching for data to satisfy the requests. When a request is satisfied, this information is written to the appropriate output files, and a statement summarizing the data retrieved is written to the standard output device. On returning to the main program, all output files and the tape drive are closed.

\subsection{Software Additions}

Several routines have not been provided with this package because they are machine dependent. The routines are gbytes, magrd, matt, and mretrn.

\subsubsection{Gbytes}

Gbytes is a general purpose bit-manipulation routine. The syntax of the subroutine gbytes is:

INTEGER IA(1), IB(1)

INTEGER IS, IL, NS, N

CALL GBYTES (IA, IB, IS, IL, NS, N)

Gbytes treats ia as a bit string. In other words, the highest order bit of $i a(1)$ is the first bit in the string and the lowest order 
bit of $i a(i)$ is considered to be contiguous with the highest order bit of $i a(i+1)$. Gbytes ignores word boundaries in $i a$.

$N$ bytes, each $i l$ bits long, are extracted from $i a$, and placed in the low order end of successive words of ib (zero padded to the left). Is bits are skipped before the first byte. Ns bits are skipped between successive bytes. Notice that byte is used here in its most general sense: a contiguous string of one or more bits.

For example, let the contents of the input array $i a$ be

$$
\mathrm{ia}=\mathrm{Z}^{\prime} 123456789 \mathrm{ABCDEF}{ }^{\prime}
$$

where the notation $Z^{\prime}$ '..' means a string of hexadecimal digits, and let the call to gbytes be

$$
\text { call gbytes(ia, ib, 10, 5, 2,7) }
$$

then the values of the output array $i b$ are:

$$
\begin{aligned}
& \mathrm{ib}(1)=\mathrm{Z}^{\prime} 001 \mathrm{~A}^{\prime} \\
& \mathrm{ib}(2)=\mathrm{Z}^{\prime} 0015^{\prime} \\
& \mathrm{ib}(3)=\mathrm{Z}^{\prime} 000 \mathrm{~F}^{\prime} \\
& \mathrm{ib}(4)=\mathrm{Z}^{\prime} 0009^{\prime} \\
& \mathrm{ib}(5)=\mathrm{Z}^{\prime} 0015^{\prime} \\
& \mathrm{ib}(6)=\mathrm{Z}^{\prime} 0013^{\prime} \\
& \mathrm{ib}(7)=\mathrm{Z}^{\prime} 001 D^{\prime}
\end{aligned}
$$

assuming that each element of $i b$ is 16 bits in length.

Gbytes is referenced in the routines filreq, getlin, and head.

Examples of gbytes for CDC, IBM, Honeywell, and some DEC machines are included later in this User's Guide. (See Appendix C).

\subsubsection{Magrd}

Magrd is an input routine for reading the day tape. Each call returns the contents of one physical record from the tape or an end-of-file condition. The syntax of the function magrd is:

INTEGER IERR, MAGRD

INTEGER IA(1)

INTEGER LEN, N

IERR = MAGRD $(L U N, N, I A$, LEN $)$ 
Magrd reads up to $n$ frames ( 8 bit bytes) from logical unit number lun and places them into $i a$. The number of frames actually read is returned in len. Magrd returns 1 on a successful read, 0 on end of file, and -1 if the read was otherwise unsuccessful. If a data record is read, magrd positions the tape after the physical record regardless of its length. If an end of file is detected, magrd positions the tape after the file mark.

Magrd is referenced in filreq, getrqt, and sum.

\subsubsection{Matt}

Matt opens the tape drive (from which the day tape will be read) for reading. The syntax for the subroutine matt is:

INTEGER NDEN, NDR, NRW

CALL MATT (NDR, NDEN, NRW)

Matt opens the logical tape unit (or physical drive) designated by $n d r$. Nden specifies the tape density. Nmw is the read/write flag, 1 for reading, 2 for writing.

The subroutine matt may need to be changed to suit your system. Matt presupposes that the user knows what logical tape unit needs to be opened. A more general usage might be to have the tape label name as an argument, and let matt return the logical tape unit in the variable ndr. In that case, the syntax of matt might be:

CHARACTER $* 6$ TAPENM

INTEGER NDR, NDEN, NRW

CALL MATT (TAPENM, NDR, NDEN, NRW)

Matt is referenced in ndtsum and retrv.

\subsubsection{Yretrn}

Mretrn is used to close the tape drive. The syntax for the subroutine mretrn is:

INTEGER NDR

CALL MRETRN (NDR)

Mretrm closes the logical tape file previously opened by matt.

Mretrm is referenced in ndtsum and retrv. 


\subsection{Additional Changes}

Additional changes may be needed to get the source code to compile and load at your site. The following paragraphs give a few suggestions of where to look for problems that may occur.

To help the user spot the machine dependencies easily in the software, the following comment has been inserted before each statement that may be machine dependent:

C*

MACHINE DEPENDENT

\subsubsection{Close Statements}

The close statement is used to remove the association between a unit number and the file or device it currently represents.

Close statements are referenced in clsout, getdat, and sample.

\subsubsection{Rewind Statements}

A rewind statement is used to set the current position of a file or device to the beginning of the file or device. If your system positions the file at the beginning when the file is opened, this rewind statement is not necessary.

The rewind statement is referenced in sample.

\subsubsection{Save Statements}

The purpose of a save statement is to retain the value of a variable or a common block after the execution of a return statement, and not initialize the variables each time the program is entered. Most implementations of FORTRAN do this automatically.

Save statements are referenced in iasnlu, irddat and irdhd.

\subsubsection{Nwords}

In all the references to magrd, the argument nwords is set equal to 1000 in a data statement. This constant depends on the word length for your machine. If the word length for your machine is nbits long, then

$$
\text { nwords }=(2000 * 8+(\text { nbits }-1)) / \text { nbits }
$$

Nwords is referenced in flreq, getrqt, and sum. 


\subsubsection{Idata}

The integer array idata is used to store one physical record from a network-day tape, 16000 bits of information. It is dimensioned to 1000 , the same as the value of nwords.

Storage for idata is provided by getrqt and sum. Idata is an argument in the routines filreq, getcal, getcpz, getlin, head, prbuf, rdntlg, and stadat, where it is declared as INTEGER IDATA (1), which is a dummy argument declaration.

\subsubsection{Constants}

Certain constants have been arbitrarily defined in the routines. They can be changed, increased or decreased, to suit the system and the users needs.

The size of the data record written on disk is set to 500 . The number 500 is referenced in filreq, and the common blocks /buffer/ and /data/.

The maximum number of user requests allowed is set to 50.50 is referenced in flsbuf, getdat, icpych, istreq, prhead, and common block / reqsts/.

\subsubsection{Colon}

The colon $(:)$ character is used in several routines in a format statement to print out the time of day. A colon may not be available on all machines.

The colon is referenced in getlin, getrqt, ndtprt, and sample.

\subsection{Bromple}

\subsubsection{Semple}

The following FORTRAN main program, sample.fortran, demonstrates the usage of the routines, once data has been extracted from a network-day tape.

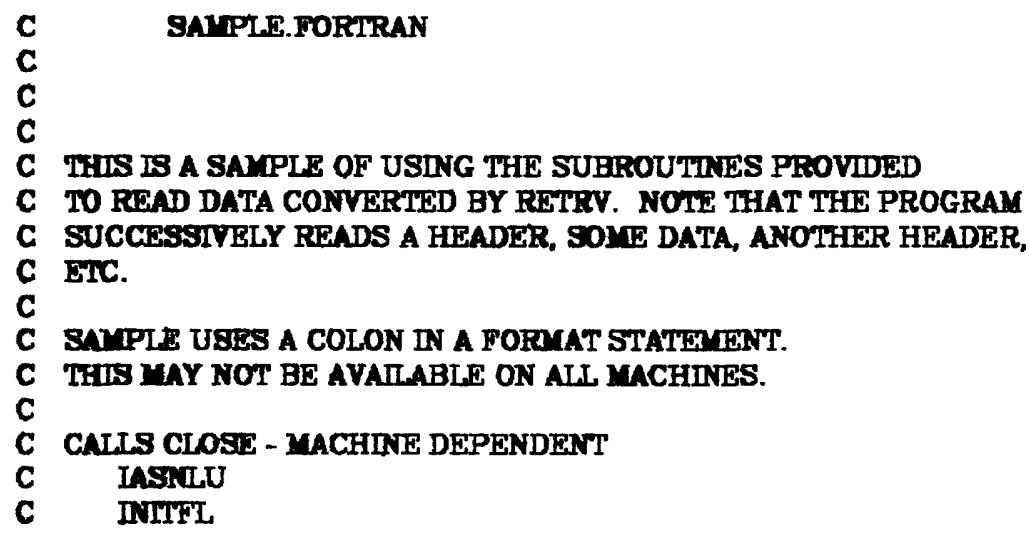




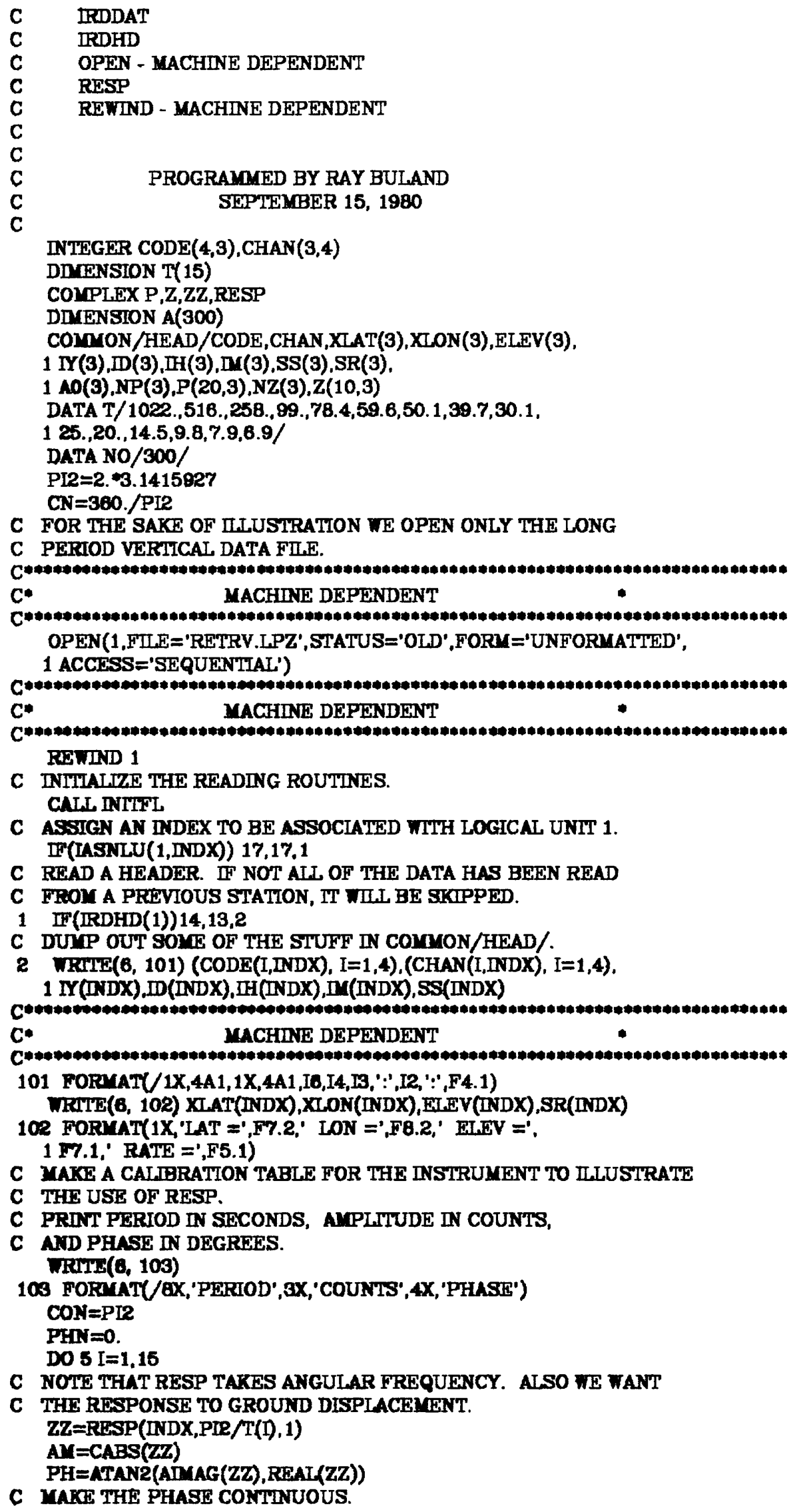


IF(ABS(PH-PHN).IE.4.) GO TO 6

CON $=$ CON-PI2

$6 \mathrm{PHN}=\mathrm{PH}$

$\mathrm{PH}=(\mathrm{PH}+\mathrm{CON}){ }^{*} \mathrm{CN}$

5 VRITE $(6,104)$ T(I),AM,PH

104 FORMAT(5X,3F9.1)

C READ UP TO THE FIRST 300 DATA POINIS AT THIS STATION.

DO $3 I=1$,NO

IF(IRDDAT(INDX,A(I))) 15,4,3

3 CONTINUE

$\mathrm{I}=\mathrm{NO}+1$

$4 I=I-1$

C DUMP THEM OUT.

WRITE $(6,105)$ I, (A(J),J=1,I)

105 FORMAT(/5X,I5/(5X,1P7E11.3))

GO TO 1

C CATCH ERRORS IN THE HEADER.

$14 \operatorname{WRTTE}(6,200)$

200 FORMAT('ERROR IN HEADER.')

GO TO 16

C CATCH ERRORS IN THE DATA.

15 WRITE $(6,201)$

201 FORMAT('ERROR IN DATA.')

GO TO 16

C DATA BUFFERS FULL.

17 WRTTE(6,20R)

202 FORMAT('DATA BUFFERS FULI.') GO TO 16

C ALL DONE.

13 WRTTE (6, 203)

203 FORMAT('ALL DATA READ.')

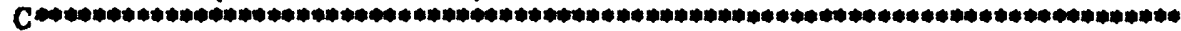

C*

MACHINE DEPENDENT

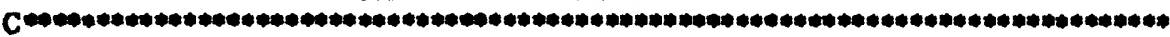

$16 \operatorname{CLOSE}(1)$

STOP

END

Using request line (A) as input to retrv, sample reads the file RETRV.LPZ and produces the output:

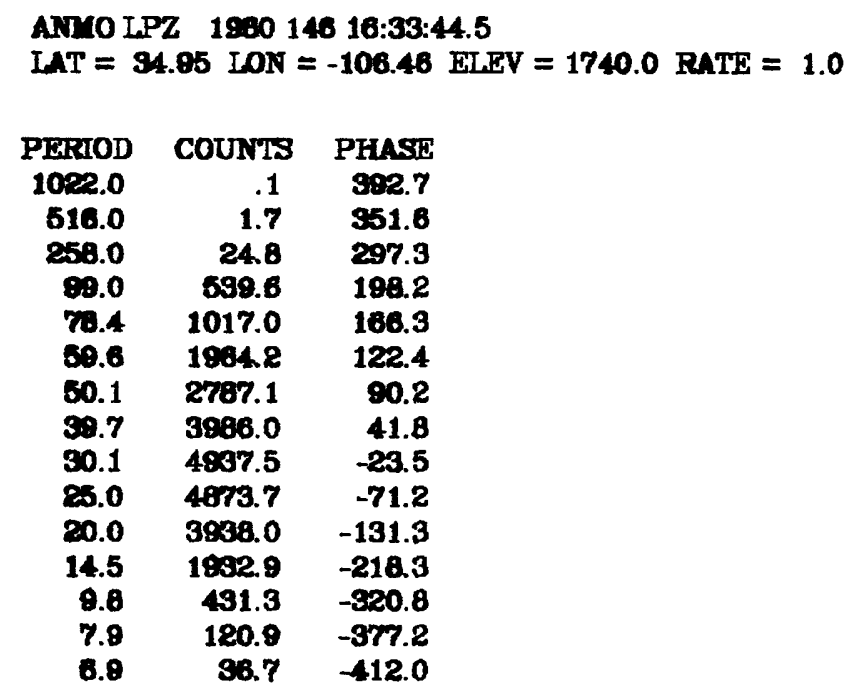


300

\begin{tabular}{|c|c|c|c|c|c|c|}
\hline 6.400E+01 & $1.010 \mathrm{E}+02$ & $1.090 \mathrm{E}+02$ & $1.130 \mathrm{E}+02$ & $1.090 E+02$ & $9.300 \mathrm{E}+01$ & $7.000 \mathrm{E}+01$ \\
\hline $4.100 E+01$ & $1.300 E+01$ & $-1.100 \mathrm{E}+01$ & $-2.900 \mathrm{E}+01$ & $-4.000 \mathrm{E}+01$ & $-4.600 \mathrm{E}+01$ & $-4.900 \mathrm{E}+01$ \\
\hline$-4.300 \mathrm{E}+01$ & $-2.900 \mathrm{E}+01$ & $7.000 \mathrm{E}+00$ & $2.300 \mathrm{E}+01$ & $5.100 \mathrm{E}+01$ & $7.500 \mathrm{E}+01$ & 8. $600 \mathrm{E}+01$ \\
\hline & $9.200 \mathrm{E}+01$ & $7.300 \mathrm{E}+01$ & $6.300 \mathrm{E}+01$ & 5.200E+01 & $4.000 \mathrm{E}+01$ & $2.200 \mathrm{E}+01$ \\
\hline 5.000E+00 & $-1.100 E+01$ & $-2.000 \mathrm{E}+01$ & $-2.400 \mathrm{E}+01$ & $-1.800 E+01$ & $-1.400 E+01$ & $-6.000 E+00$ \\
\hline$-7.000 E+00$ & $-9.000 \mathrm{E}+00$ & $-9.000 \mathrm{E}+00$ & $-4.000 E+\infty 0$ & 4.000E+00 & $1.500 \mathrm{E}+01$ & $2.300 \mathrm{E}+01$ \\
\hline & $2.600 \mathrm{E}+01$ & $2.300 \mathrm{E}+01$ & $2.000 \mathrm{E}+01$ & $1.600 \mathrm{E}+01$ & $1.000 \mathrm{E}+01$ & $\mathrm{OE}+01$ \\
\hline & $2.000 \mathrm{E}+01$ & $3.400 \mathrm{E}+01$ & $4.800 \mathrm{E}+01$ & $5.800 \mathrm{E}+01$ & $6.300 \mathrm{E}+01$ & $E+01$ \\
\hline & $4.100 E+01$ & 2.700E+01 & $1.900 \mathrm{E}+01$ & $00 \mathrm{E}+01$ & $100 \mathrm{E}+01$ & $\mathrm{DE}+00$ \\
\hline & $a E+01$ & $\mathrm{OE}+01$ & $-4.400 E+01$ & $10 E+01$ & $00 E+01$ & $0 E+01$ \\
\hline$O E+01$ & $-5.700 \mathrm{E}+01$ & $-5.600 \mathrm{E}+01$ & $-5.700 \mathrm{E}+01$ & $-6.300 E+01$ & $-8.600 E+01$ & $-6.900 E+01$ \\
\hline $\mathrm{OE}+01$ & $E+01$ & $\mathrm{E}+01$ & $-6.900 \mathrm{E}+01$ & $-7.000 E+01$ & $-6.600 \mathrm{E}+01$ & $\mathrm{OE}+01$ \\
\hline+01 & $\mathrm{OE}+01$ & $\mathrm{E}+01$ & $-3.600 \mathrm{E}+01$ & $0 O E+01$ & $\mathrm{E}+01$ & $E+01$ \\
\hline $0 E+00$ & $2.200 E+01$ & $3.500 \mathrm{E}+01$ & $3.800 \mathrm{E}+01$ & $100 E+01$ & $\mathrm{O} \mathrm{E}+\infty \mathrm{O}$ & $\mathrm{DE}+01$ \\
\hline$-3.500 \mathrm{E}+01$ & $-4.200 \mathrm{E}+01$ & $\mathrm{OE}+01$ & $-3.800 \mathrm{E}+01$ & $\mathrm{OE}+01$ & $\mathrm{OE}+01$ & $E+01$ \\
\hline $4.500 \mathrm{E}+01$ & $-4.500 E+01$ & $\mathrm{OE}+01$ & $-3.800 \mathrm{E}+01$ & $\mathrm{OE}+01$ & $\mathrm{OOE}+01$ & $O E+01$ \\
\hline+01 & $O E+01$ & $00 E+01$ & $-4.800 E+01$ & $O E+01$ & $20 E+01$ & $E+01$ \\
\hline+01 & $\mathrm{OE}+01$ & $\mathrm{E}+01$ & $-4.000 x+\infty 0$ & $O E+01$ & $\mathrm{OE}+01$ & $E+01$ \\
\hline+01 & $D E+01$ & $E+01$ & $\mathrm{OE}+01$ & $0 E+01$ & $\mathrm{OEE}+01$ & $3 \mathrm{E}+01$ \\
\hline$-6.800 \mathrm{E}+01$ & $00 E+01$ & $O E+01$ & $100+01$ & $O E+01$ & $a E+01$ & $\mathrm{E}+01$ \\
\hline$-1.000 \mathrm{E}+01$ & $00 \mathrm{E}+01$ & $O E+01$ & $O E+01$ & $\mathrm{OE}+\mathrm{O}$ & $\mathrm{OE}+01$ & $O E+00$ \\
\hline$-1.600 \mathrm{E}+01$ & $-2.500 \mathrm{E}+01$ & $-3.100 E+01$ & $\mathrm{OE}+01$ & $O E+01$ & $O E+01$ & $\mathrm{DE}+01$ \\
\hline+01 & $O E+01$ & +01 & $O E+0 R$ & $70 \mathrm{E}+02$ & $10 E+02$ & $3.7202+02$ \\
\hline 6.07 & $5 E+03$ & $E+03$ & $2.698 \mathrm{E}+03$ & $48 E+03$ & $32 E+03$ & $3 E+03$ \\
\hline 0.48 & $6 \mathrm{E}+04$ & +04 & $3 E+04$ & $E+0$ & $\mathrm{OE}+\mathrm{CB}$ & $1 \mathrm{E}+03$ \\
\hline$-1.403 \mathrm{~B}+03$ & $-6.344 \mathrm{E}+03$ & $-1.0995+04$ & $-1.480 \mathrm{E}+04$ & $-1.774 \mathrm{E}+04$ & $392+04$ & $86 \mathrm{E}+04$ \\
\hline$-1.839 \mathrm{E}+04$ & $-1.837 \mathrm{E}+04$ & $-1.714 E+04$ & $-1.600 \mathrm{E}+04$ & $-1.53 \mathrm{AE}+04$ & $-1.502 E+04$ & $-1.514 \mathrm{E}+04$ \\
\hline$-1.526 \mathrm{E}+04$ & $192+04$ & $30 E+04$ & $-1.26 \mathrm{PE}+04$ & $-9.936 E+03$ & $-6.264 E+03$ & $-1.813 \mathrm{E}+03$ \\
\hline 2.84 & $2 A E+03$ & $5 E+04$ & $1.502 \mathrm{E}+04$ & 1.7RgE+04 & 1.842E +04 & 1.88EE +04 \\
\hline 1.80 & $25+04$ & $025+04$ & $2.22025+04$ & $2.493 E+04$ & $\mathrm{CPE}+04$ & $4 \mathrm{E}+04$ \\
\hline $3.470 E+04$ & $3.670 \mathrm{E}+04$ & $3.630 \mathrm{E}+04$ & $3.4602+04$ & $3.010 \mathrm{E}+04$ & 2.301E+04 & $1.363 \mathrm{E}+04$ \\
\hline $2.490 E+03$ & $-1.001 E+04$ & $-2.2801 E+04$ & $-3.574 E+04$ & $-4.78: E+04$ & $-6.827 \mathrm{E}+04$ & $-6.707 \mathrm{E}+04$ \\
\hline$-7.88 .65+04$ & -7.84aE +04 & $-8.0905+04$ & -8.12XE +04 & $-7.855 E+04$ & $-7.605 E+04$ & $-7.078 \mathrm{~F}+04$ \\
\hline$-6.4131+04$ & $-5.828 \mathrm{E}+04$ & $-4.746 E+04$ & $-3.80215+04$ & $-2.658 \mathrm{dE}+04$ & $-1.831 E+04$ & $-1.086 \mathrm{E}+04$ \\
\hline$-2.856 \mathrm{~g}+03$ & colon & & & & & $1.431 \mathrm{~F}+04$ \\
\hline $1.916 \mathrm{gg}+04$ & $1.140 \mathrm{E}+04$ & $9.404 E+03$ & $7.432 \mathrm{et}+03$ & $5.516 \mathrm{E}+03$ & $3.864 \mathrm{E}+\infty 3$ & $2.516 \mathrm{E}+03$ \\
\hline $1.5542+03$ & $1.084 \mathrm{E}+03$ & 1.023:5+03 & $1.28020+00$ & $1.600 \mathrm{E}+09$ & $2.458 \mathrm{AE}+03$ & $3.016 \mathrm{E}+03$ \\
\hline $3.378 E+09$ & 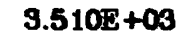 & $3.4545+03$ & $3.270 \mathrm{E}+\infty 3$ & $3.090 \mathrm{E}+03$ & $3.114 \mathrm{E}+03$ & $3.5062+03$ \\
\hline $4.500 \mathrm{~B}+03$ & $5.55 \mathrm{AP}+03$ & $7.2324+03$ & 9.224E+03 & 1.130tet & $1.351 E+04$ & $1.551 \mathrm{E}+04$ \\
\hline 1. resiet+ut & 1.850E +04 & 1. $0285+04$ & $1.8802+04$ & $1.8875+04$ & $1.739 \mathrm{E}+04$ & $1.557 \mathrm{E}+04$ \\
\hline 1.320E+04 & $1.074 E+04$ & B. 13aE+03 & $5.584 \mathrm{E}+03$ & 3.164E+03 & 9.690E + 02 & $-9.690 E+02$ \\
\hline -2.TOLE+03 & $-4.270 E+03$ & $-5.464 \mathrm{~F}+03$ & $-6.212 \mathrm{E}+03$ & $-6.390 \mathrm{D}+03$ & $-5.876 \mathrm{E}+03$ & $-4.612 \mathrm{2E}+03$ \\
\hline$-2.6168+03$ & $-4.600 \mathrm{~L}+01$ & $2.8445+03$ & $5.688 \mathrm{E}+03$ & 8.130E +03 & $8.836 \mathrm{E}+03$ & \\
\hline
\end{tabular}

ALC DATA READ.

\section{Notea}

There are no prompts in the commands ndtsum and retrv to tell the user what information he needs to enter next.

Retru normally reads requests from the standard input device. Another alternative is to insert these requests in a file, and have retru read from this file. To do this, the subroutine getdat.fortran needs to be changed. Note the comments that have been inserted in getdat.fortran to assist you in doing this.

If your machine swaps bytes (eg. PDP11's and VAX's), you will need to add a routine to magrd that exchanges even and odd bytes of the data. 


\section{Table 1. Summary of Routines}

\begin{tabular}{|c|c|c|}
\hline Routine & Description & $\begin{array}{l}\text { Referenced } \\
\text { by }\end{array}$ \\
\hline close & $\begin{array}{l}\text { statement } \\
\text { opens a flle or device }\end{array}$ & $\begin{array}{l}\text { clsout } \\
\text { sample }\end{array}$ \\
\hline clsout & $\begin{array}{l}\text { subroutine } \\
\text { write a null header to each open } \\
\text { output file before closing it }\end{array}$ & retrv \\
\hline daymo & $\begin{array}{l}\text { integer function } \\
\text { converts day of year to month and } \\
\text { day }\end{array}$ & $\begin{array}{l}\text { getrqt } \\
\text { ndtprt }\end{array}$ \\
\hline doy & $\begin{array}{l}\text { integer function } \\
\text { converts month and day to day of } \\
\text { year }\end{array}$ & req \\
\hline dtpfmt & $\begin{array}{l}\text { real function } \\
\text { decodes binary data from Network } \\
\text { Day Tape }\end{array}$ & filreq \\
\hline ffin & $\begin{array}{l}\text { real function } \\
\text { scans character string for } \\
\text { numeric constant }\end{array}$ & $\begin{array}{l}\text { getcal } \\
\text { getcpz } \\
\text { getdat } \\
\text { stadat }\end{array}$ \\
\hline flireq & $\begin{array}{l}\text { subroutine } \\
\text { fills a request }\end{array}$ & getrqt \\
\hline fisbuf & $\begin{array}{l}\text { subroutine } \\
\text { writes data buffers to output files }\end{array}$ & filreq \\
\hline gbytes & $\begin{array}{l}\text { subroutine } \\
\text { general purpose bit manipulation } \\
\text { routine }\end{array}$ & $\begin{array}{l}\text { filreq } \\
\text { getlin } \\
\text { head }\end{array}$ \\
\hline getcal & $\begin{array}{l}\text { integer function } \\
\text { scans data log for calibration } \\
\text { constants }\end{array}$ & getrqt \\
\hline getcpz & $\begin{array}{l}\text { subroutine } \\
\text { scans data log for station } \\
\text { constants, poles, and zeroes }\end{array}$ & getrqt \\
\hline
\end{tabular}


Table 1. Summary of Routines

Routine Description

$\begin{array}{ll}\text { getdat } & \begin{array}{l}\text { subroutine } \\ \text { scans input request file }\end{array} \\ \text { getlin } & \begin{array}{l}\text { subroutine } \\ \text { reads characters from input } \\ \text { buffer, then converts them to } \\ \text { the internal character code of } \\ \text { the host machine }\end{array}\end{array}$

getrqt subroutine

reads Network Day Tape searching

for data that will satisfy a

request

getstr

subroutine

scans a character string for a

blank or comma-delimited substring

head

subroutine

decodes Network Day Tape header information

iasnlu integer function

associates a logical unit number

with an index in the data arrays

icpych subroutine

copies one character string to

another

initbd subroutine

initializes output files, logical

unit numbers, and instrument codes

initfl subroutine

initializes header and data

record flags
Referenced

by

retrv

getcal

getcpz

getrqt

prbuf

rdntlg

stadat

sum

retrv

getcal

getdat

rdntlg

stadat

filreq

getrqt

sum

sample

getdat

retrv

sample 
Table 1. Summary of Routines

Routine Description

irddat integer function

reads next data point of a data record

irdhd

integer function

reads next header record

istreq integer function

compares two character strings

lpyr integer function

determines if year is a leap year

magrd function

reads day tape returning one

physical record

matt subroutine

opens tape drive

mod intrinsic function

returns remainder

mretrn subroutine

closes tape drive

ndtprt subroutine

write out values in common

blocks /start/ and /end/

ndtsum command

dump contents of a Network Day Tape

open

statement

opens a file

opnout subroutine

opens binary output files
Referenced

by

irdhd

sample

sample

getrqt

req

setflg

daymo

doy

stndrd

filreq

getrqt

sum

ndtsum

retrv

lpyr

ndtsum

retrv

sum

opnout

sample

retrv 
Table 1. Summary of Routines

\begin{tabular}{|c|c|c|}
\hline Routine & Description & $\begin{array}{l}\text { Referenced } \\
\text { by }\end{array}$ \\
\hline prbuf & $\begin{array}{l}\text { subroutine } \\
\text { writes out data logs }\end{array}$ & sum \\
\hline prhead & $\begin{array}{l}\text { subroutine } \\
\text { writes out a header record to a } \\
\text { binary output file }\end{array}$ & filreq \\
\hline rddata & $\begin{array}{l}\text { subroutine } \\
\text { reads a data record }\end{array}$ & irddat \\
\hline rdhead & $\begin{array}{l}\text { subroutine } \\
\text { reads a header record }\end{array}$ & irdhd \\
\hline rdntlg & $\begin{array}{l}\text { subroutine } \\
\text { scans a data log }\end{array}$ & getrqt \\
\hline req & $\begin{array}{l}\text { integer function } \\
\text { determines if current record will } \\
\text { fill a request }\end{array}$ & getrqt \\
\hline resp & $\begin{array}{l}\text { complex function } \\
\text { returns the complex forward } \\
\text { transfer function of the } \\
\text { instrument system }\end{array}$ & sample \\
\hline retrv & $\begin{array}{l}\text { command } \\
\text { retrieves selected information } \\
\text { from a Network Day Tape }\end{array}$ & \\
\hline rewind & $\begin{array}{l}\text { statement } \\
\text { positions file at the beginning }\end{array}$ & sample \\
\hline sample & $\begin{array}{l}\text { command } \\
\text { example program }\end{array}$ & \\
\hline save & $\begin{array}{l}\text { statement } \\
\text { retains values of variable after } \\
\text { execution of return statement }\end{array}$ & $\begin{array}{l}\text { iasnlu } \\
\text { irddat } \\
\text { irdhd }\end{array}$ \\
\hline setfig & $\begin{array}{l}\text { subroutine } \\
\text { sets request station flags }\end{array}$ & $\begin{array}{l}\text { getrqt } \\
\text { rdntlg }\end{array}$ \\
\hline
\end{tabular}


Table 1. Summary of Routines

Routine Description

stadat subroutine

scans a data log

stndrd subroutine

checks time values for consistency

sum subroutine

produces summary of a Network

Day Tape

tset subroutine

sets time variables
Referenced

by

getrqt

filreq

sum

ndtsum

filreq

sum 
Table 2. Summary of Common Blocks

Common Block Description

/buffer/

/data/

/end/

/files/

/flags /

/head/

station and start time information

flags that signal what type of record is to be read next from disk

contains three arrays for each

orienation of data

stores binary data read from disk

end time of continuous data

output files and instrument codes

/header/

Network Day Tape header information

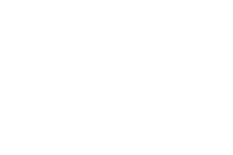

/reqsts/

user request information

Referenced

by

filreq

flsbuf

getrqt

retrv

initfl

irddat

rddata

ndtprt

ndtsum

sum

clsout

getdat

initbd

opnout

retrv

iasnlu

initfl

irddat

irdhd

irdhd

rdhead

resp

filreq

getrqt

head

ndtsum

prhead

req

retrv

sum

tset

filreq getdat getrqt 


\section{Table 2. Summary of Common Blocks}

\section{Common Block Description}

/start/

/sstart/

/stinfo/

req
retrv
setflg

start time of continuous data

start time of request

station information
Referenced

by

ndtprt

ndtsum

sum

filreq getrqt prhead retrv

filreq getrqt prhead retrv 
Table 3. Routines Used by Main Programs

\author{
Main
}

Routine

close

clsout

daymo

doy

dtpfmt

ffin

filreg

flsbuf

gbytes $\uparrow$

getcal

getcpz

getdat

getlin

getrat

getstr

head

iasnlu

icpych

initbd

initf

irddat

irdhd

istreq

\begin{tabular}{|c|c|c|}
\hline Retrv & Ndtsum & Sample \\
\hline$*$ & & $*$ \\
\hline \multicolumn{3}{|l|}{ * } \\
\hline$*$ & * & \\
\hline \multicolumn{3}{|l|}{$*$} \\
\hline \multicolumn{3}{|l|}{$*$} \\
\hline \multicolumn{3}{|l|}{$*$} \\
\hline \multicolumn{3}{|l|}{$*$} \\
\hline \multicolumn{3}{|l|}{$*$} \\
\hline$*$ & $*$ & \\
\hline \multicolumn{3}{|l|}{$*$} \\
\hline \multicolumn{3}{|l|}{$*$} \\
\hline \multicolumn{3}{|l|}{$*$} \\
\hline$*$ & $*$ & \\
\hline \multicolumn{3}{|l|}{$*$} \\
\hline \multicolumn{3}{|l|}{$*$} \\
\hline \multirow[t]{2}{*}{$*$} & $*$ & \\
\hline & & * \\
\hline \multicolumn{3}{|l|}{$*$} \\
\hline \multicolumn{3}{|l|}{$*$} \\
\hline & & $*$ \\
\hline & & $*$ \\
\hline & & * \\
\hline * & & \\
\hline & & \\
\hline
\end{tabular}


Table 3. Routines Used by Main Programs

\begin{tabular}{|c|c|c|c|}
\hline \multirow{2}{*}{ Routine } & \multicolumn{3}{|c|}{ Main } \\
\hline & Retrv & Ndtsum & Sample \\
\hline Ipyr & $*$ & $*$ & \\
\hline magrd + & * & $*$ & \\
\hline matt + & $*$ & $*$ & \\
\hline $\bmod$ & $*$ & $*$ & \\
\hline mretrn $\dagger$ & $*$ & $*$ & \\
\hline ndtprt & & * & \\
\hline open & $*$ & & $*$ \\
\hline opnout & $*$ & & \\
\hline prbuf & & * & \\
\hline prhead & $*$ & & \\
\hline rddata & & & $*$ \\
\hline rdhead & & & $*$ \\
\hline rdntlg & $*$ & & \\
\hline req & $*$ & & \\
\hline resp & & & $*$ \\
\hline rewind & & & * \\
\hline save & & & $*$ \\
\hline setflg & $*$ & & \\
\hline stadat & $*$ & & \\
\hline stndrd & $*$ & $*$ & \\
\hline sum & & $*$ & \\
\hline tset & $*$ & $*$ & \\
\hline
\end{tabular}

$\overline{\text { †ser-supplied routine. }}$ 
INCLUDE FILES

DEFINES.H

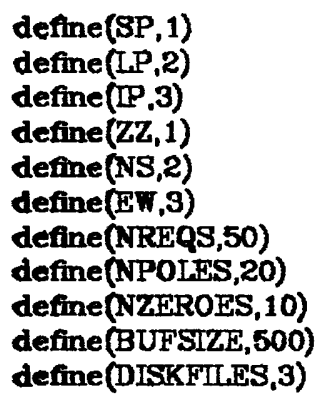

BUFFER.DCL

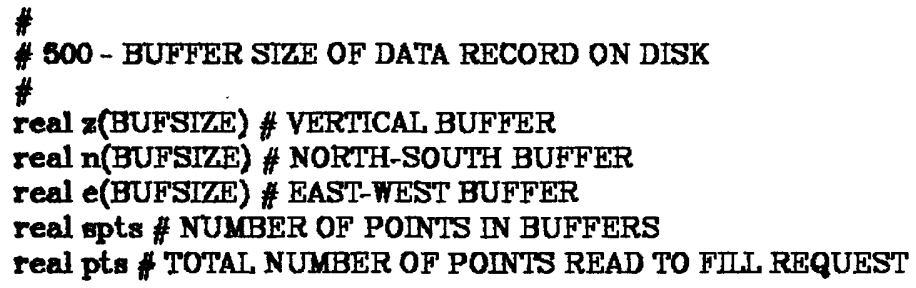

BUFFER.COM

common /buffer/z, n, e, spts, pts

DATA.DCL

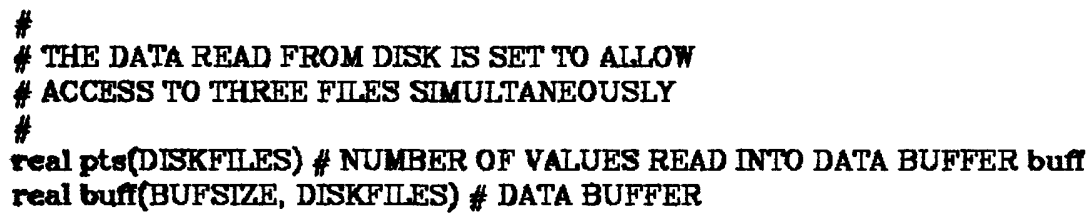

DATA.COY

*

common /data/ pts, buff

FUIES.DCL

\# integer 92 lun(3,3) \# LOGICAL UNIT NUMBER FOR EACH OUTPUT FILE integer 2 chn $(3,3,4)$ \# INSTFRULENT CODE FOR EACH OUTPUT FIl,E 


\section{APPENDIX A}

\section{RATFOR CODE}

The software written to read the Network Day Tapes was written in Ratfor (Rational FORTRAN) $\uparrow$. Ratfor translates easily into FORTRAN. The FORTRAN code produced in Appendix B was generated from this Ratfor code.

† Kernighan, Brian W. and P.J. Plauger, Software Tools, Addison-Wesley, Reading, Mascachusetts, (1978). 
FIIES.COM

\title{
$\#$
}

common /files/lun, chn

FLAGS.DCL

\begin{abstract}
$\#$
integer $\approx$ hffg(DISKFILES) \# HEADER RECORD FLAG

integer 2 dfig(DISKFILES) \# DATA RECORD FIAG

integer 2 nlun \# NUMBER OF LOGICAL UNIT NUMBERS ASSIGNED

integer 2 llun(20) \# LOGICAL UNIT NUMBERS ASSOCIATED WITH DATA INDEXES

integer'? ilun(DISKFILES) \# INDEX ASSOCIATED WITH A LOGICAL UNIT NUMBER

integer*2 npts(DISKFIIES) \# INDEX OF NEXT DATA VALUE TO BE RETURNED
\end{abstract}

FLAGS.COM

\#

common /flags/hfig, dflg, nlun, llun, ilun, npts

HEAD.DCL

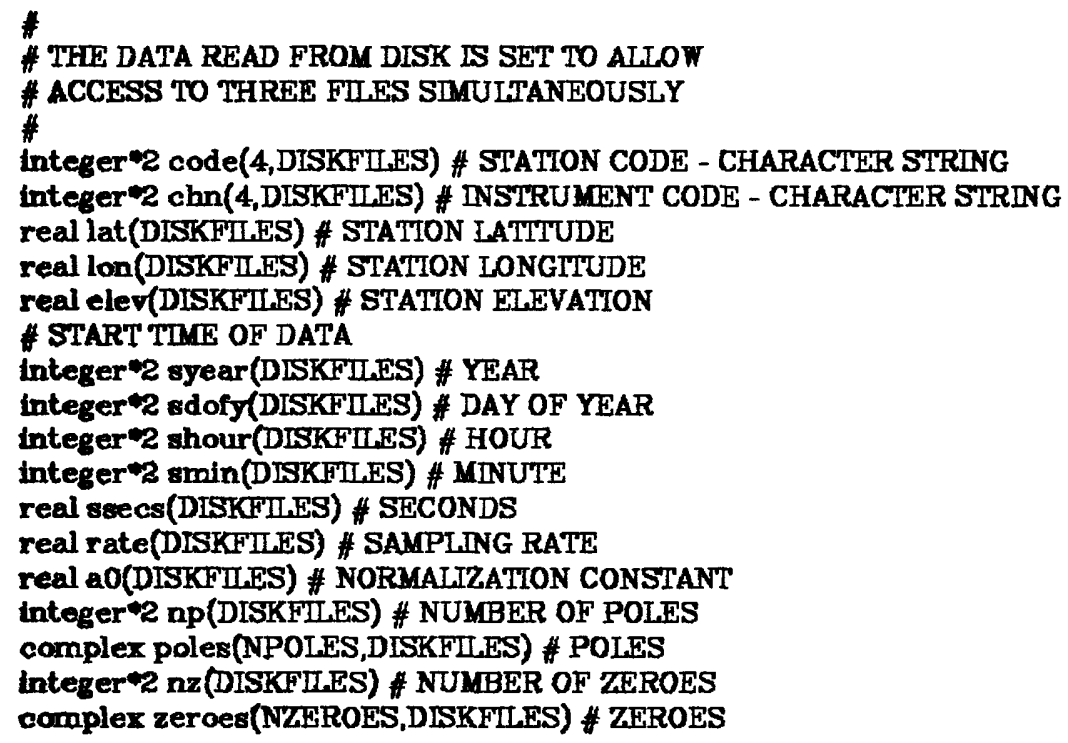

HEAD.COM

\#

common /head/ code, chn, lat, lon, elev, syear, edofy, shour, smin, ssecs, rate, a0, $\mathrm{np}$, poles, $\mathrm{nz}$, zeroes 
HFADER.DCL

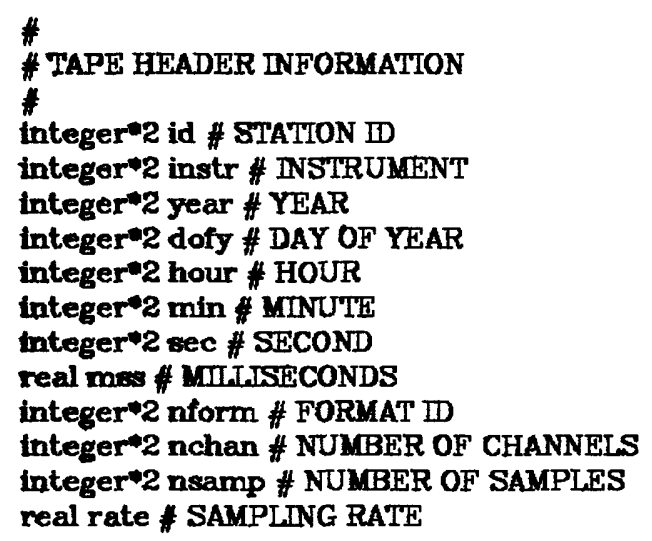

HEADER.COM

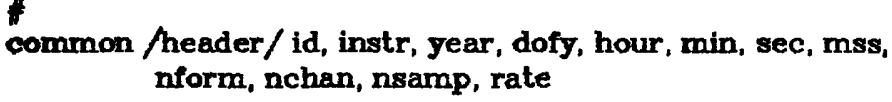

REQUESTS.DCL

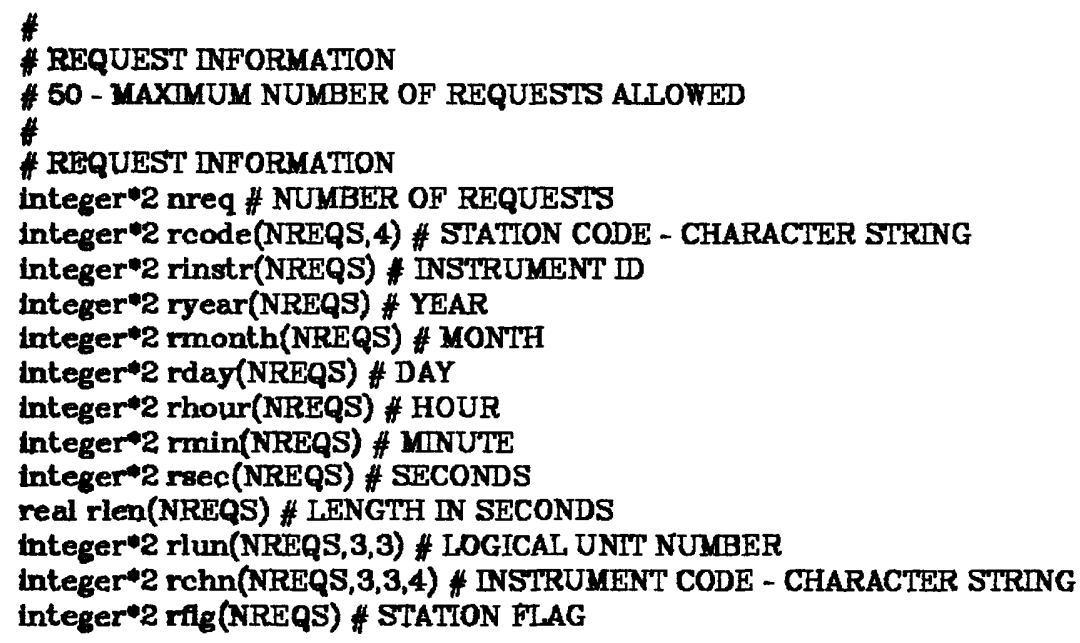

REQUESTS.COM

STAINFO.DCL

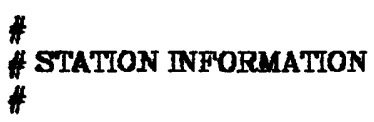


integer $* 2$ code(4) \# STATTON CODE - CHARACTER STRING

integer $2 n p$ \# NUMBER OF POLES, 20 LARGEST NUMBER OF SRO POLES

integer 2 nz \# NUMBER OF ZEROES, 10 IARGEST NUMBER OF SRO ZEROES

integer 2 ichan \# NUMBER OF CHANNEIS

real aO(3) \# CONSTANT

real cal(3) \# CALIBRATION CONSTANT

real elev \# STATTON EILEVATION

real lat \# STATION L.ATTUDE

real lon \# STATTON LONGTTUDE

complex poles(NPOLES,3) \# POLES

complex reroes(NZEROES,3) \# ZEROES

$\#$

* START TIME OF DATA

\#

integer"2 syear \# YEAR

integer" 2 sdofy \# DAY OF YEAR

integer"2 shour \# HOUR

integer 2 amin \# MINUTES

real esecs \# SECONDS

STADNFO.COM

\author{
\# \\ common /stinfo/ code, ichan, np, nz, lat, lon, elev, ao, cal, \\ poles, zeroes \\ common /sstart/syear, sdofy, shour, smin, ssecs
}

TIMES.DCL

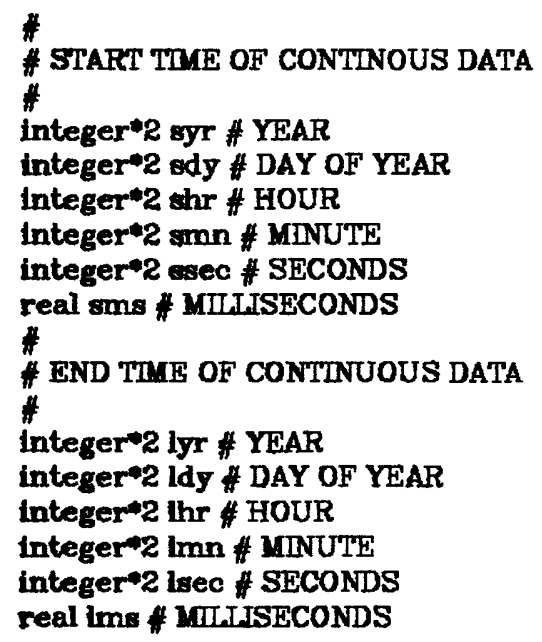

H common /start/ syr, sdy, shr, smn, ssec, sms \# common /end/ lyr, ldy, lhr, lmn, lsec, Ims 


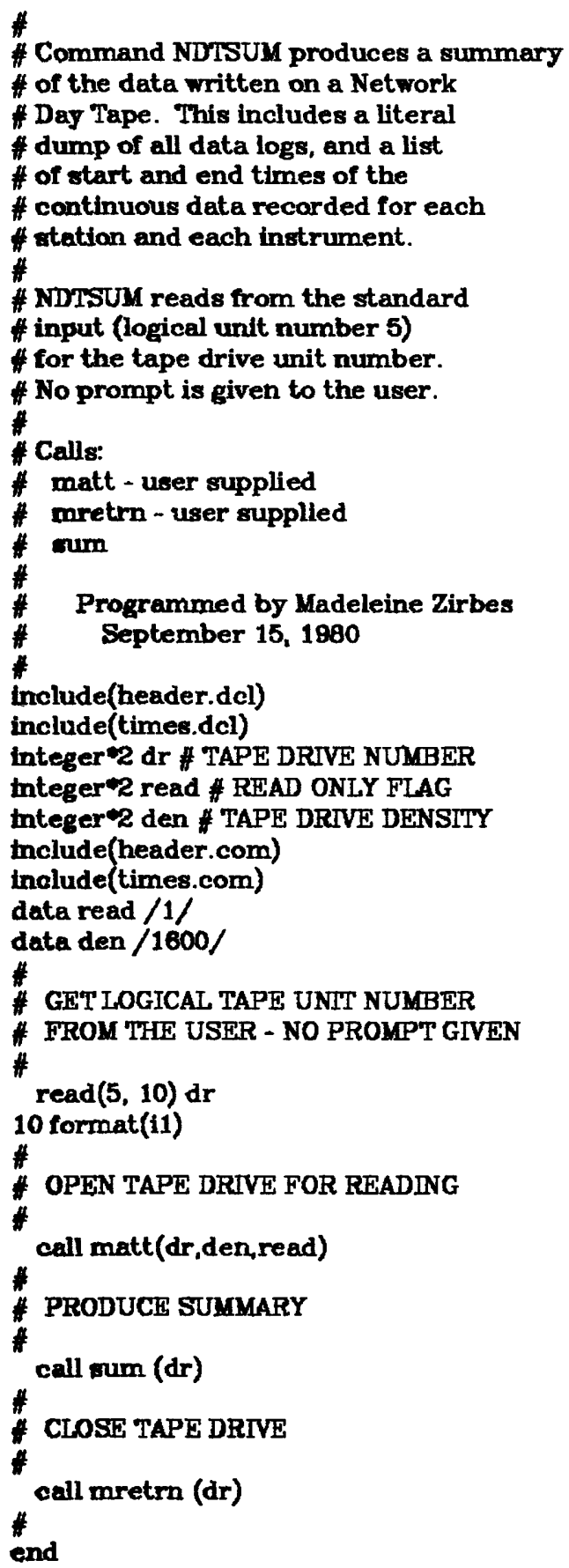


NDSTUM SPECIFIC ROUTINES

SUM.RATFOR

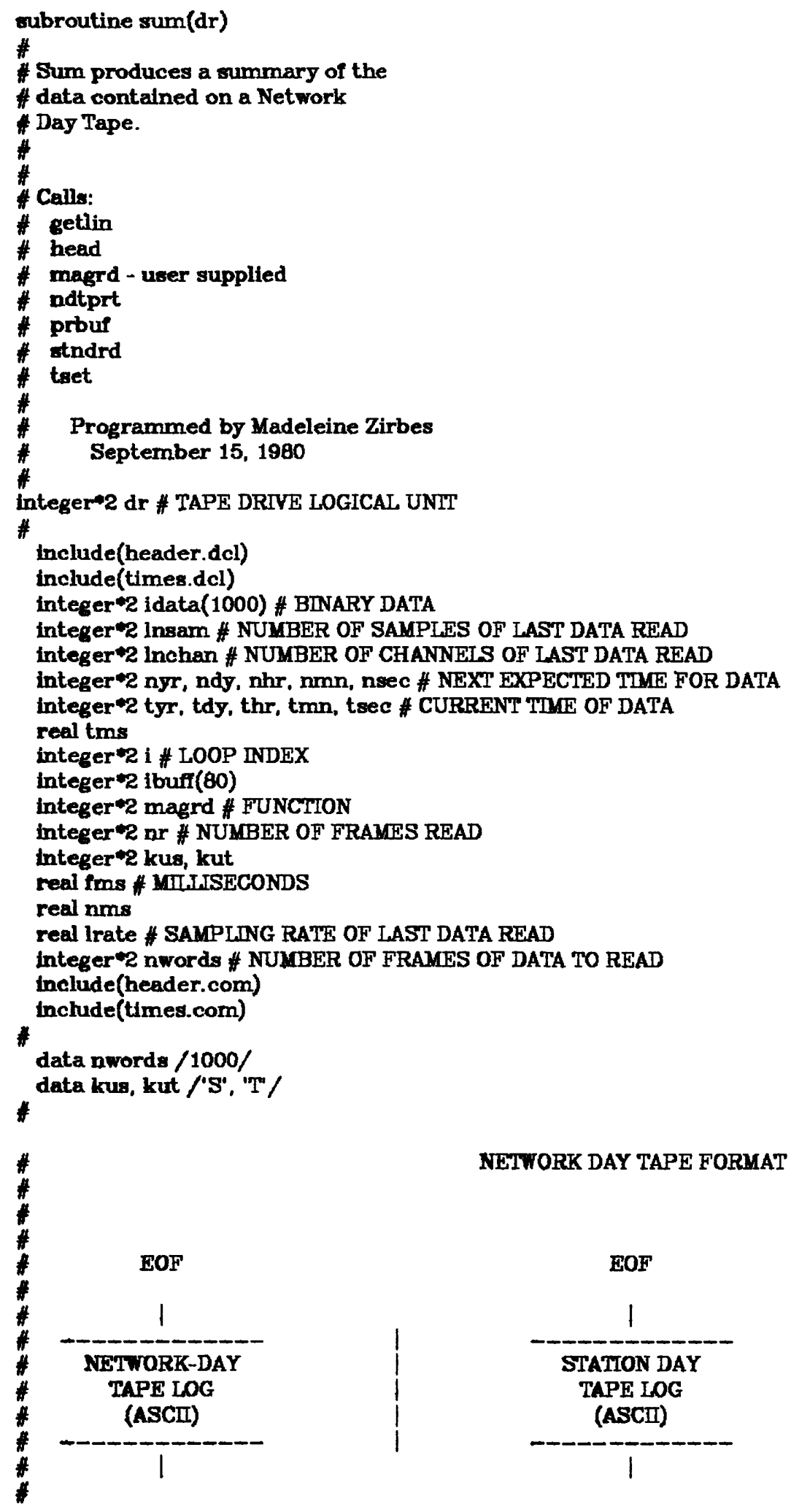

IPP DATA LOG (ASCII) 


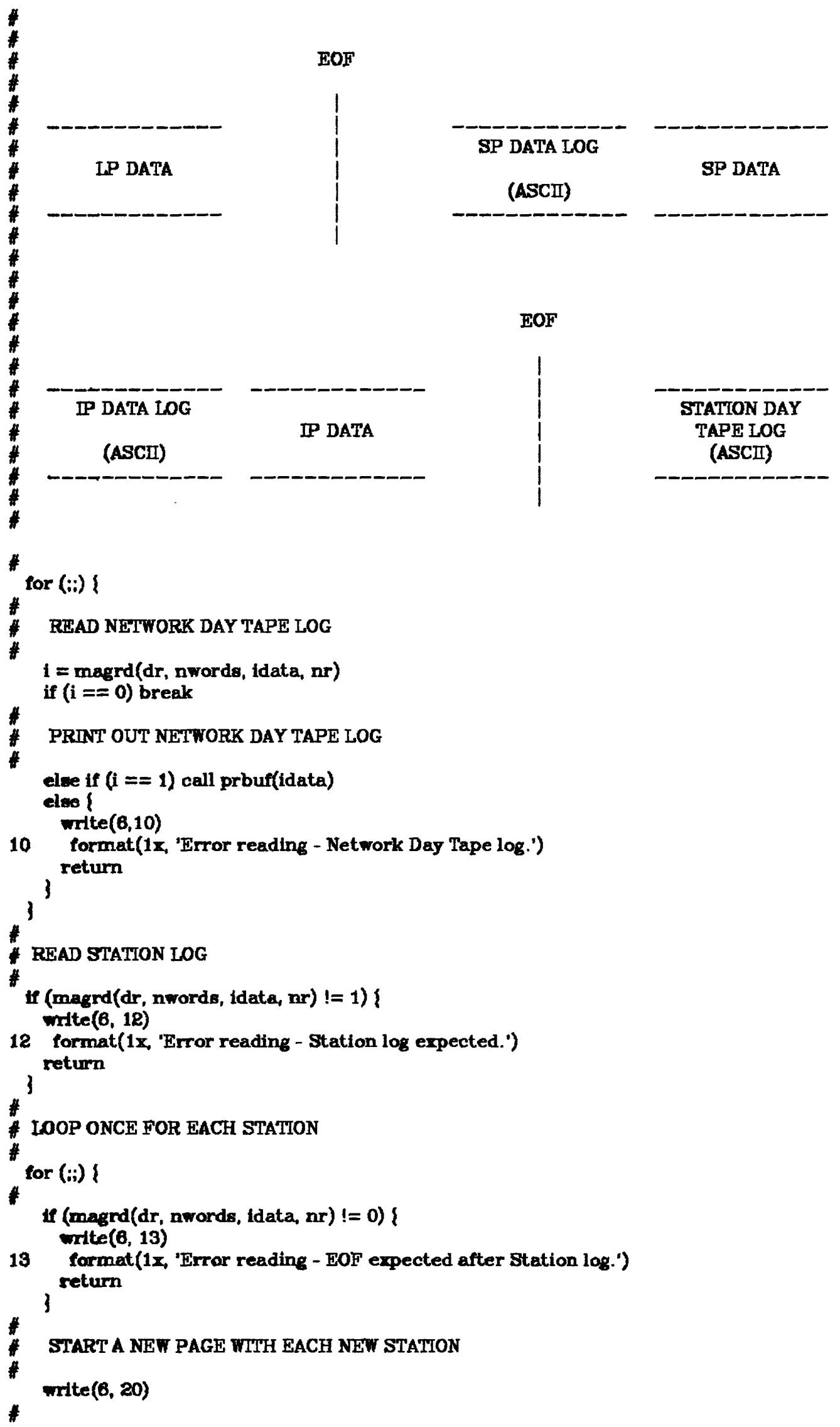




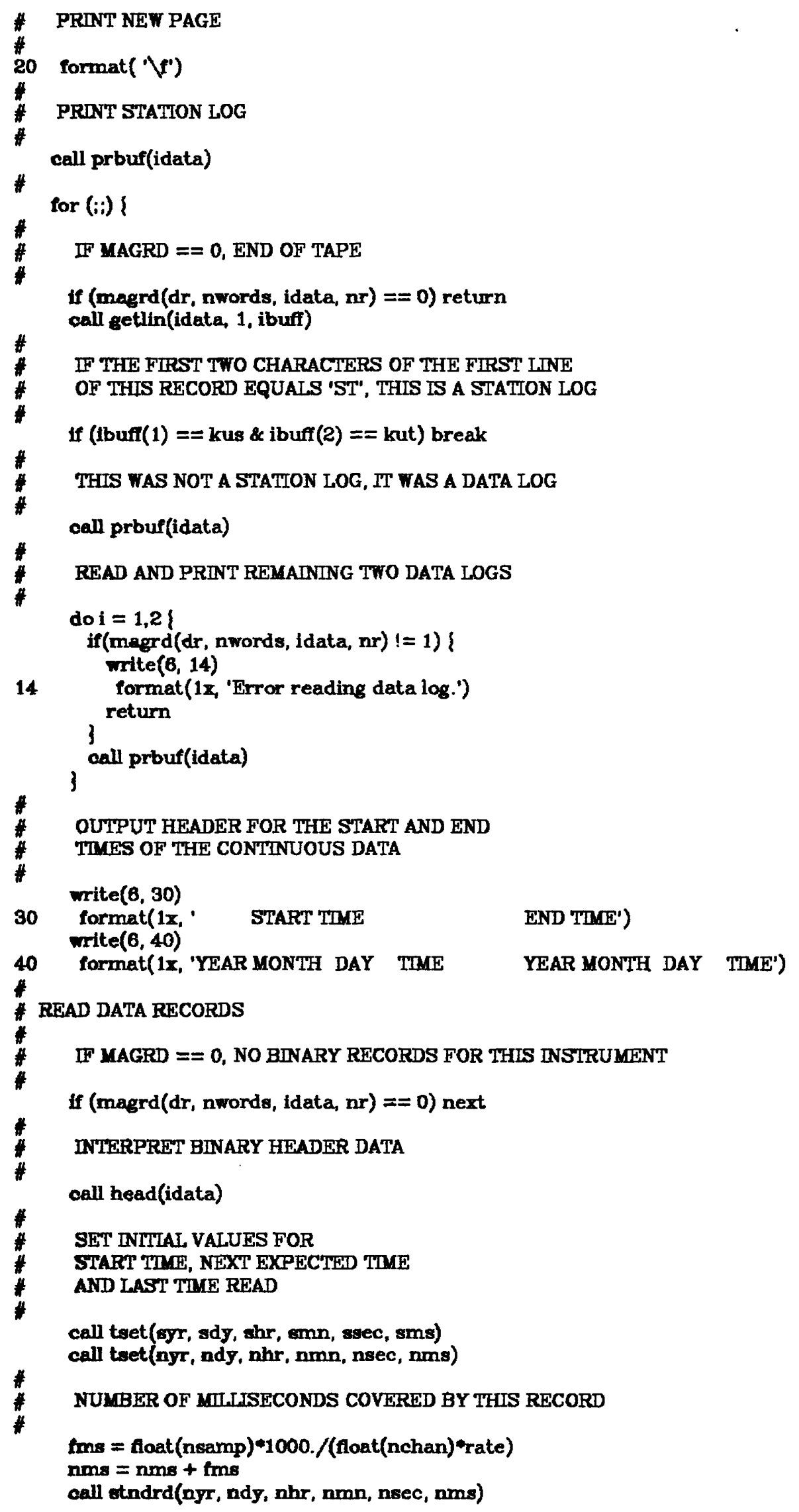


call tset(lyr, Idy, Ihr, Imn, Isec, Ims)

Insam = nsamp

lrate $=$ rate

Inchan = nchan

for(:i) (

IF MAGRD $==0$, END OF FILE

If (magrd(dr, nwords, idata, $n r)==0$ ) break call head(idata)

SET CURRENT TTME

call twet(tyr, tdy, thr, tmn, tsec, tms)

IS CURRENT TME EQUAL TO NEXT EXPECTED TTME?

if $(($ tyr $==n y r) \&($ tdy $==n d y) \&$

(thr = = nhr) \& (tmn = = nmn) \&

$($ tsec $==$ nsec $) \&($ tms $==n m s))\}$

\section{YES}

call tset(nyr, ndy, nhr, nmn, nsec, nms)

$f m s=$ foat $($ nsamp $) * 1000 . /$ (float $($ nchan $){ }^{*}$ rate $)$ \# reintialize values

$n m s=n m s+$ fms

call stndrd(nyr, ndy, nhr, nmn, nsec, nms)

call teet(lyr, Idy, lhr, Imn, lsec, Ims)

Insam $=$ nsamp

Irate $=$ rate

)

Inchan = nchan

$\#$
$\#$
$\#$
$\#$
$\#$
$\#$
$\#$
$\#$
$\#$
$\#$
$\#$
$\#$
$\#$

clse ?

NO

frns $=($ float $($ Insam $/$ Inchan $)-1.0) * 1000 . /$ rate

Ims $=\operatorname{lms}+\mathrm{fms}$

call stndrd(lyr, ldy, lhr, lmn, lsec, Ims)

PRINT OUT STARTING AND ENDING TIMES

OF THE CONTINUOUS DATA

call ndtprt

RETNITIATIZE VALUES

call teet(syr, sdy, shr, smn, ssec, sms)

call tset(nyr, ndy, nhr, nmn, nsec, nms)

fms $=$ foet (nsamp) ${ }^{*} 1000 . /\left(\right.$ float (nchan) ${ }^{\star}$ rate $)$

$\mathrm{nms}=\mathrm{nms}+\mathrm{fms}$

call atndrd(nyr, ndy, nhr, nmn, nsec, nms)

call teet(lyr, ldy, lhr, Imn, lsec, Ims)

insam = nsamp

Irate $=$ rate

3

Inchan $=$ nchan

fms $=(\text { float }(\text { Insam } / \text { Inchan })-1.0)^{* 1000} . /$ Arate

Ims = lms + fms

call stndrd(yyr, Idy, Ihr, Imn, lsec, Ims)

call ndtprt

write $(8,50)$ 
$-41-$

$3_{\text {end }}^{50, \text { format }(/ /)}$ 
PRBUF.RATFOR

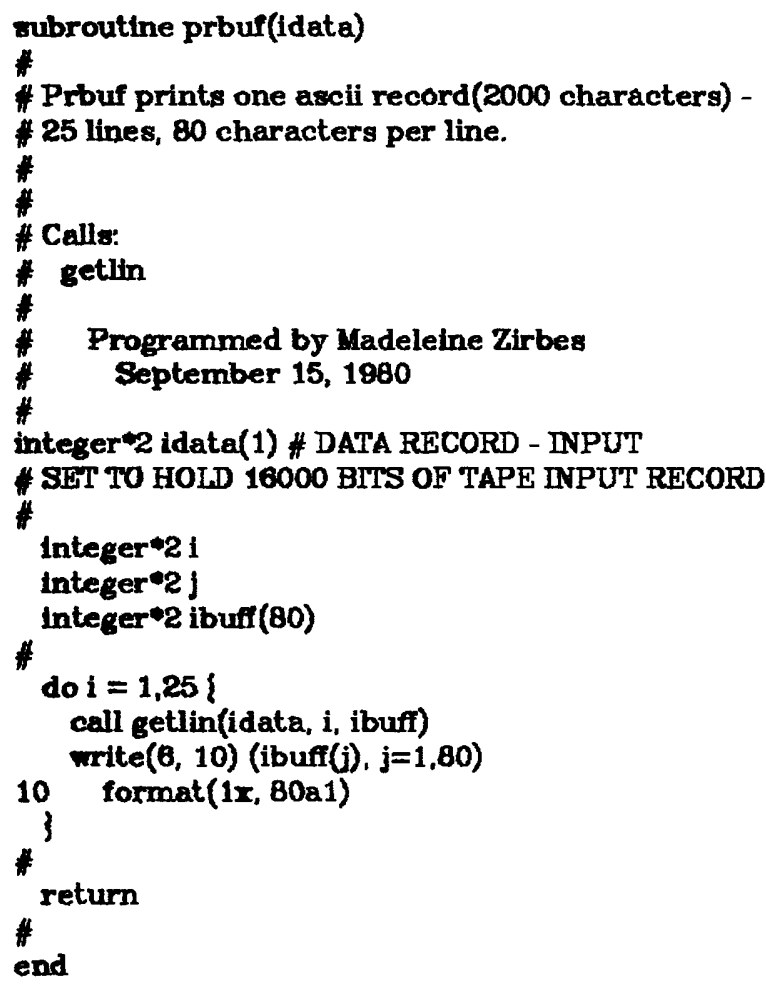




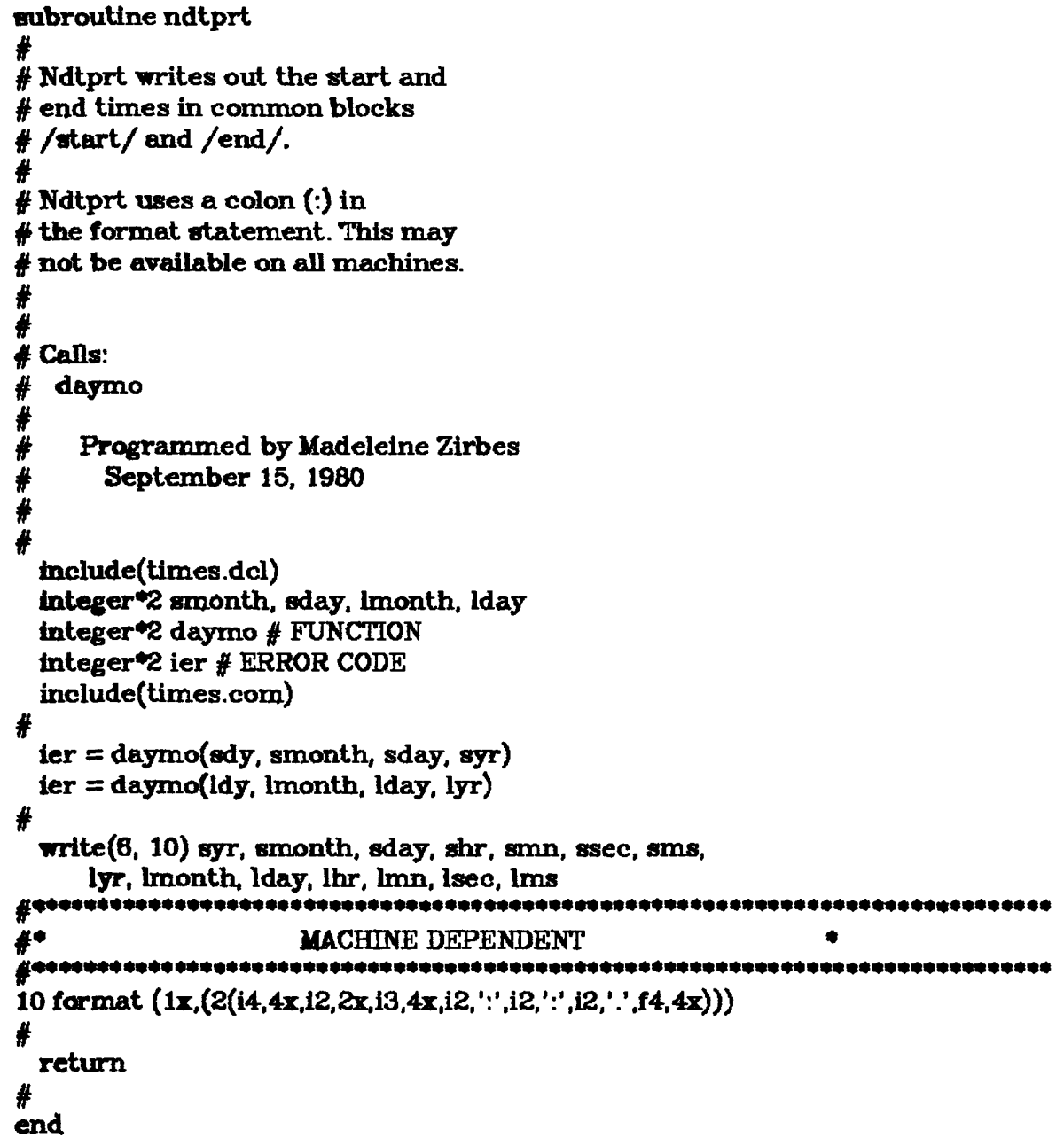




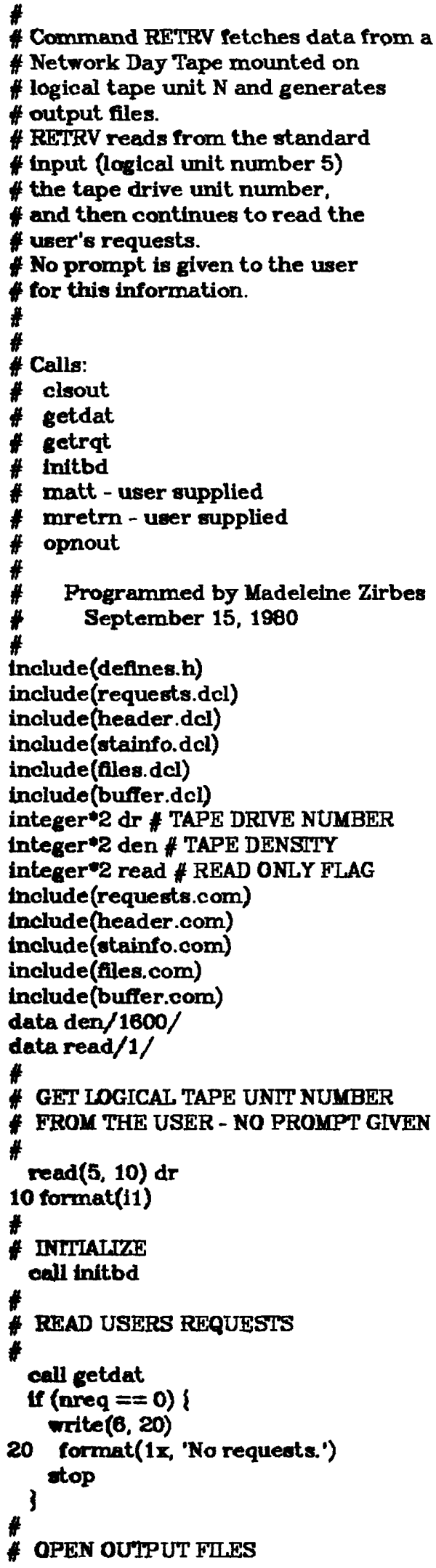




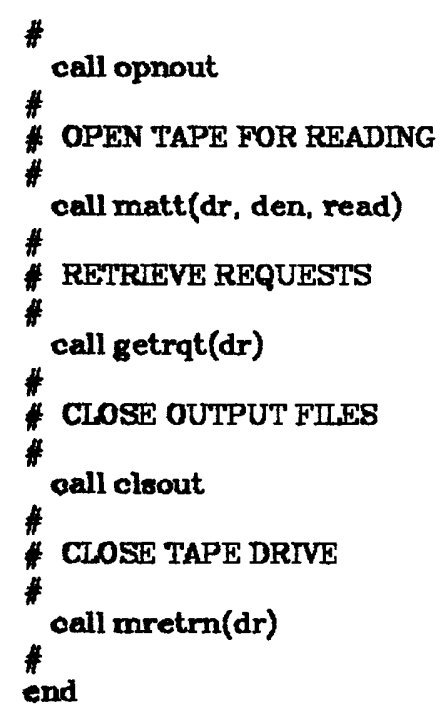


RETRV SPECIFTC ROUTINES

INITBD.RATFOR

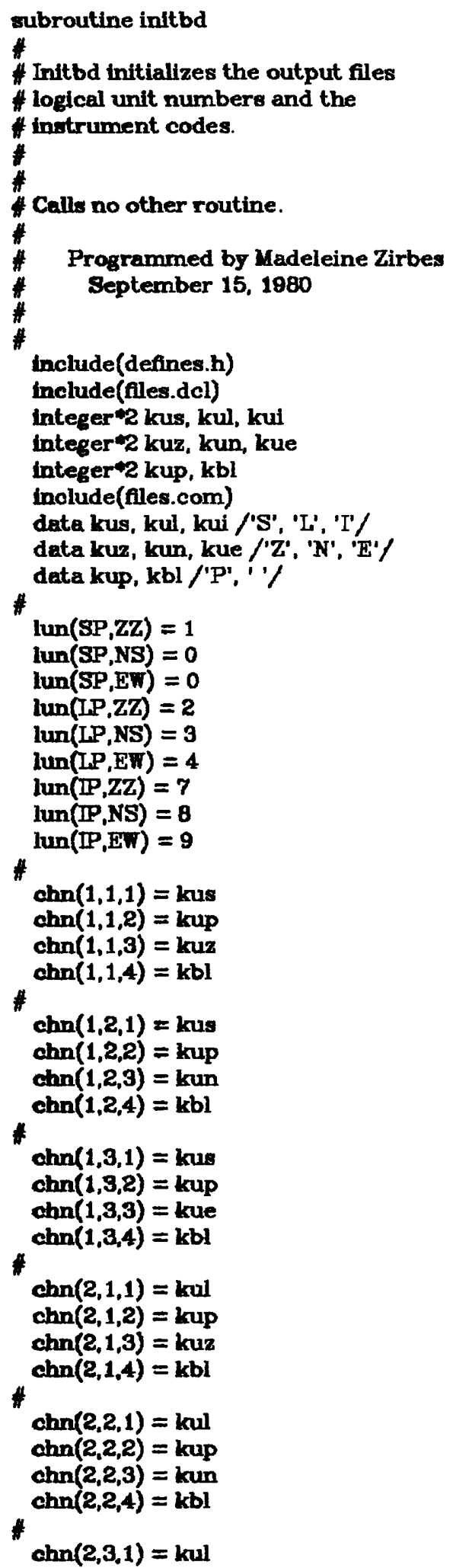




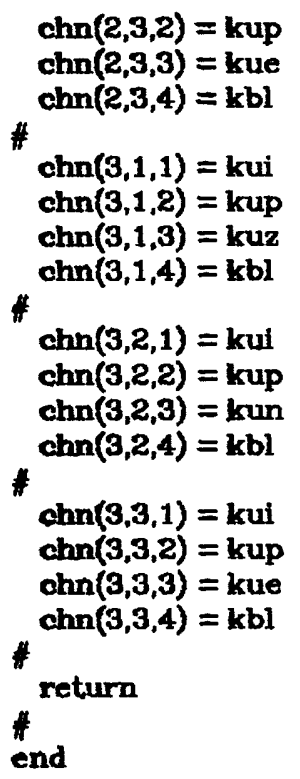


GETDAT.RATFOR

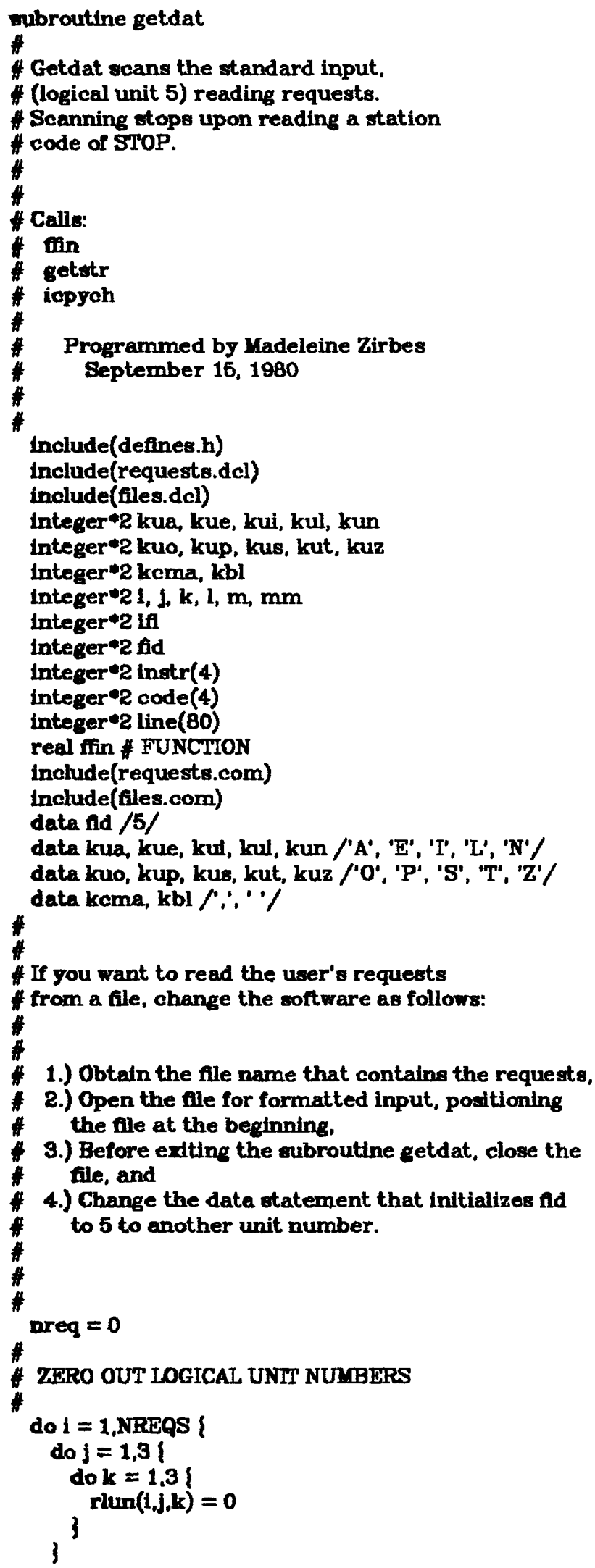




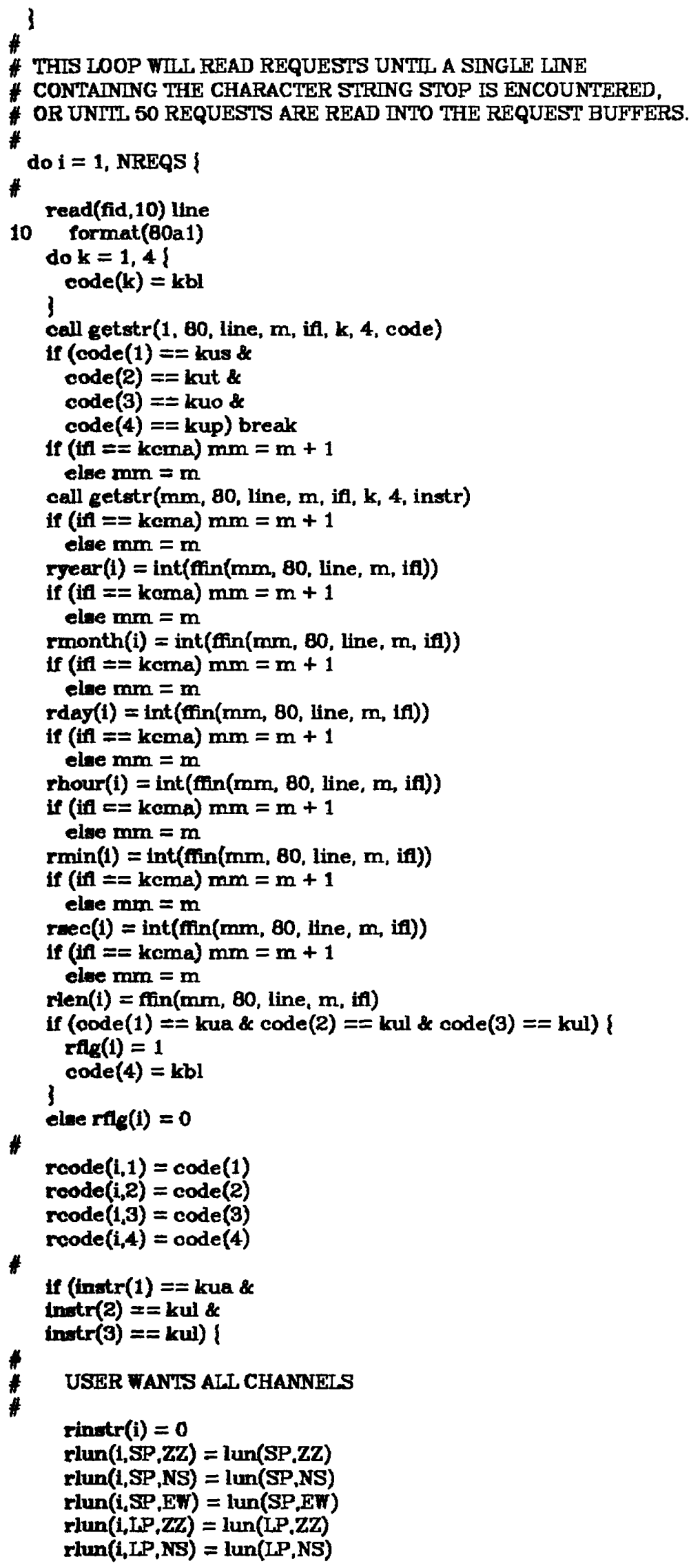




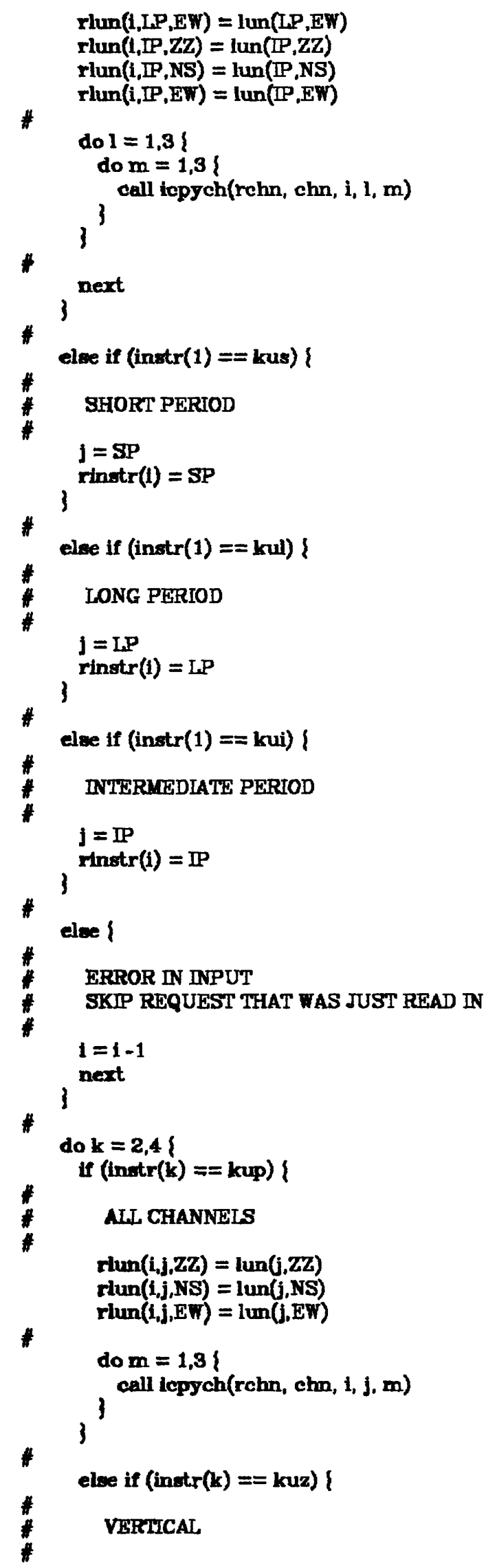




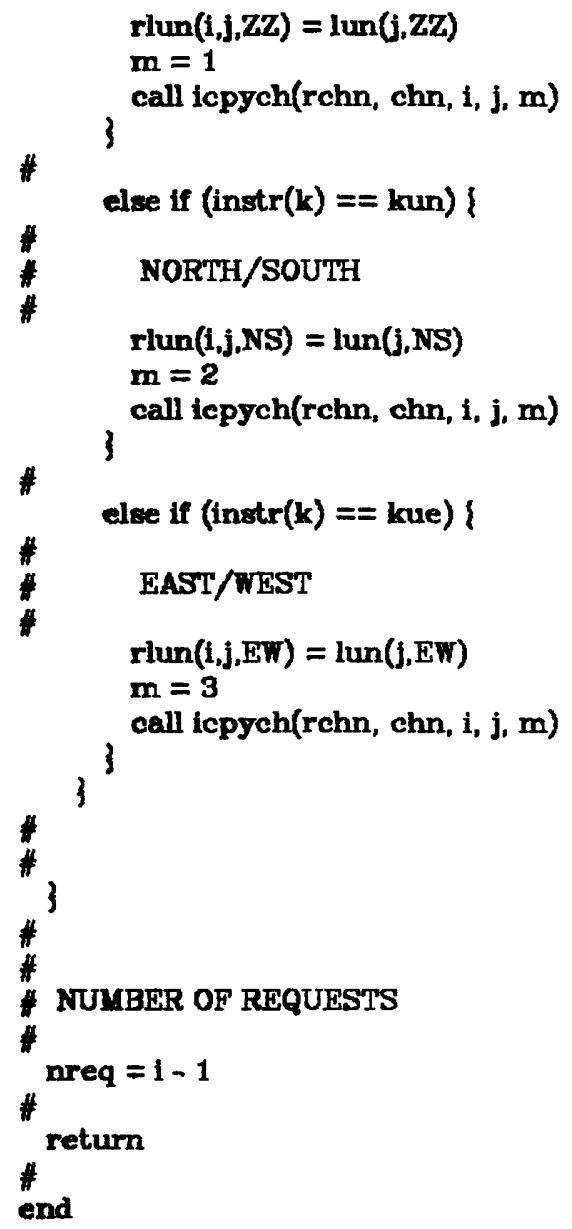




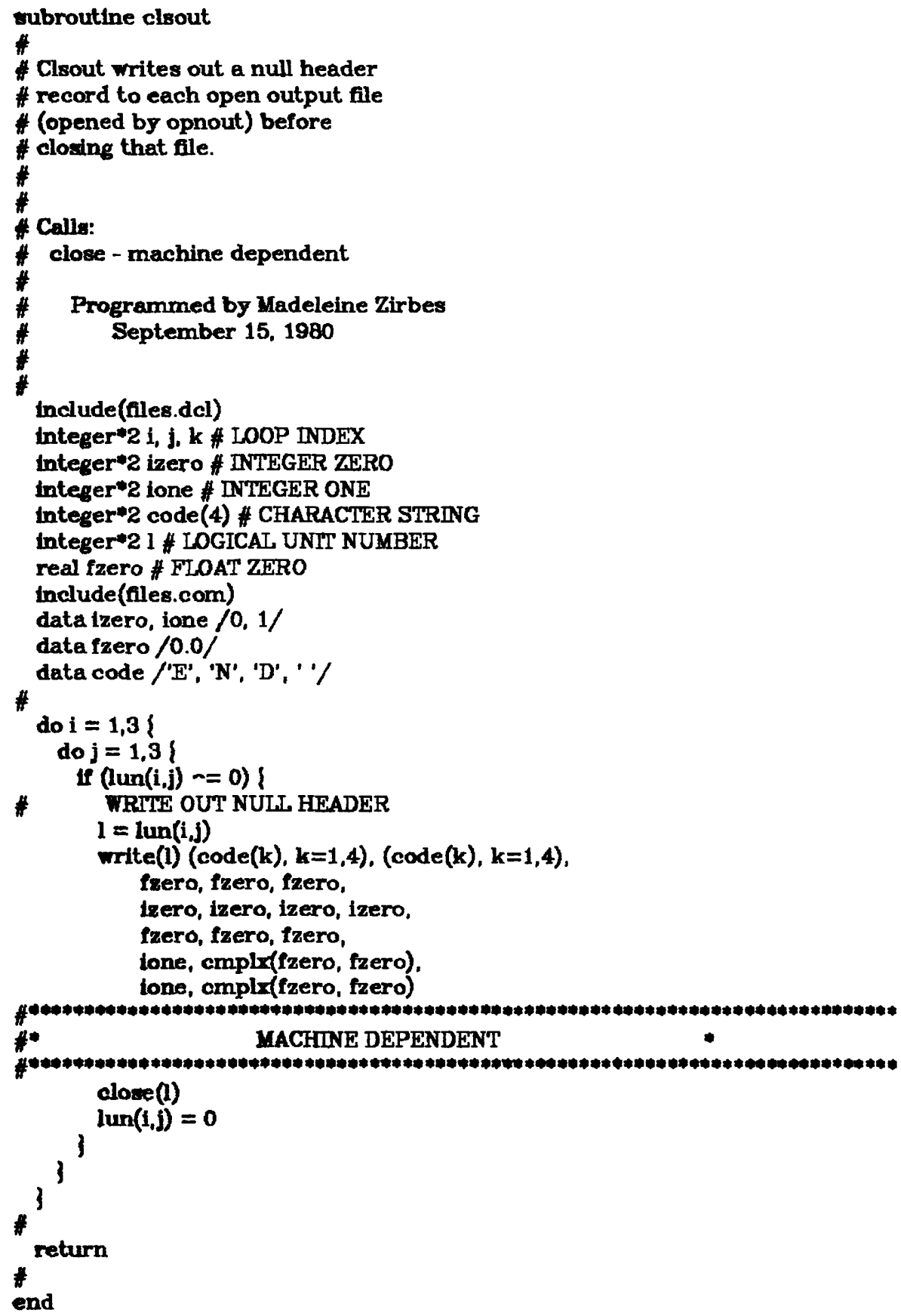


OPNOUT.RATFOR

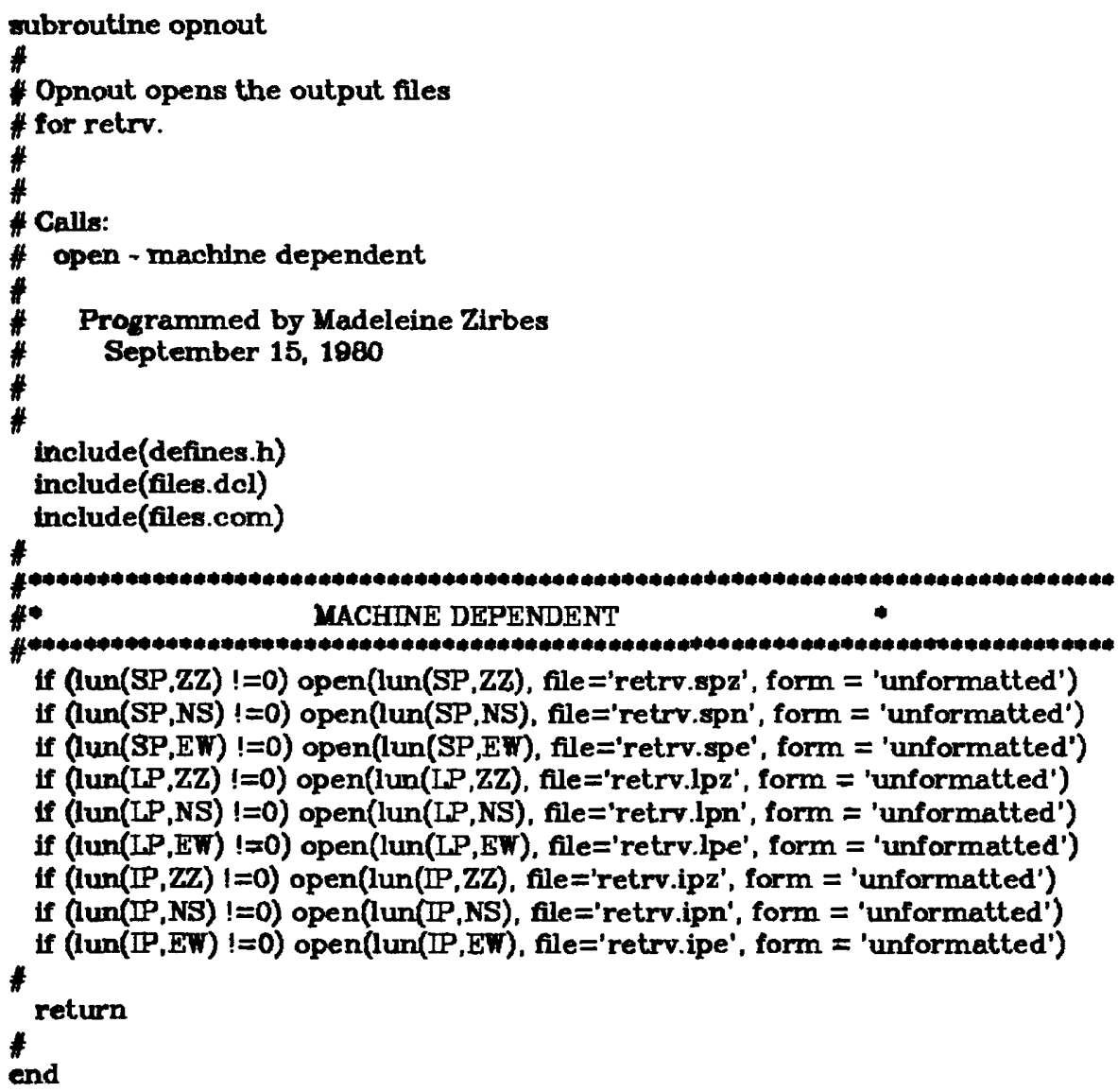




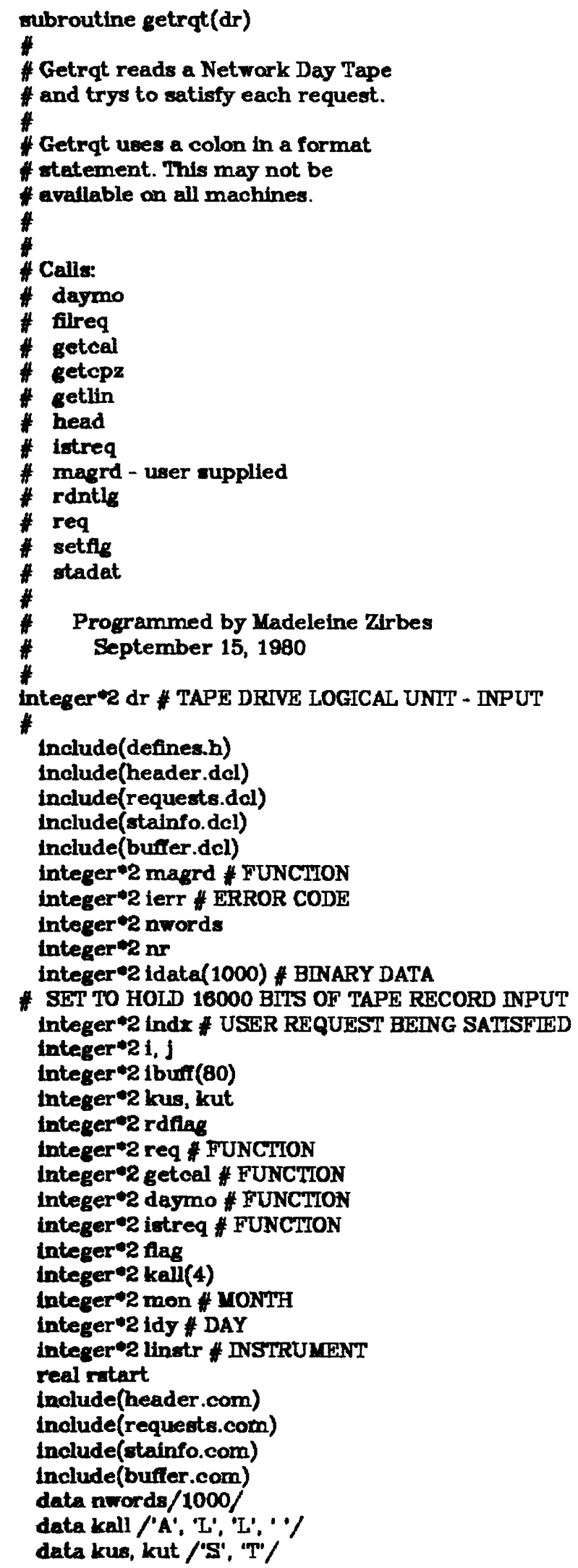


data code $/,, \cdots, \cdot, \%$

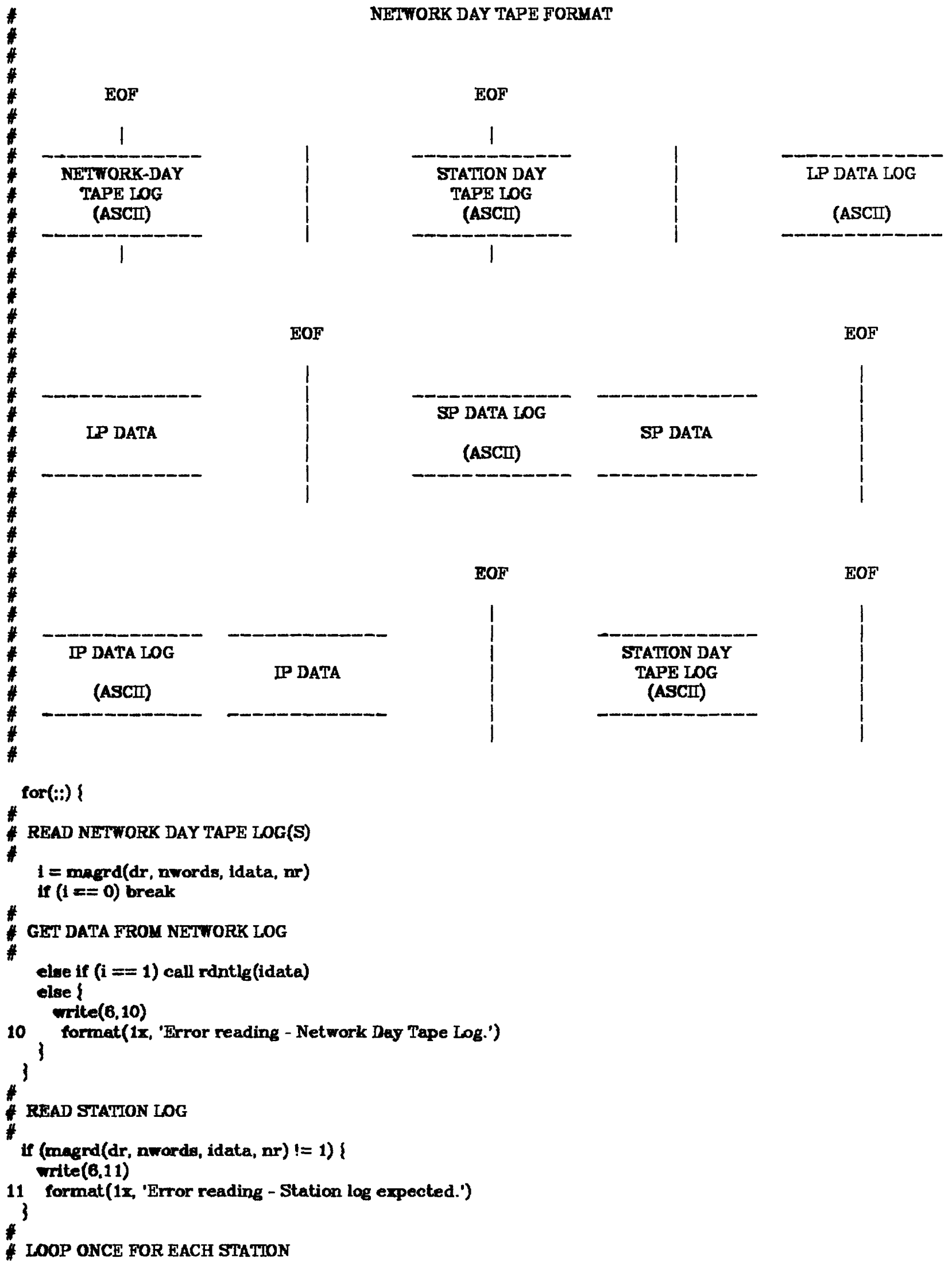




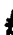
for (ii) !

\#

If (magrd(dr, nwords, idata, $n r) !=0)$ | write $(6,12)$

12 )

format (1x, 'Error reading - EOF expected after station log.')

BEFORE READING NEW STATHON LOG,

CHECK TO SEE IF THERE ARE ANY

MORE REQUESTS TO SATTSFY.

IF NOT, RETURN TO YAIN PROGRAM.

SET REQUEST FIAG TO TRUE FOR ALL REQUESTS THAT

HAVE THE REQUESTED STATTON EQUAL TO 'ALL'

call setifg(kall, 1)

\#

SET REQUEST FIAG TO FAISE FOR ALL REQUESTS THAT

MATCH THE PREVIOUS STATTON CODE. EVEN IF THEY

WERE NOT SATISFIED, NO MORE DATA FOR THAT STATTON

REMAINS ON THE TAPE.

call setfig(code, 0 )

$\#$

ARE THERE ANY OUTSTANDING REQUESTS?

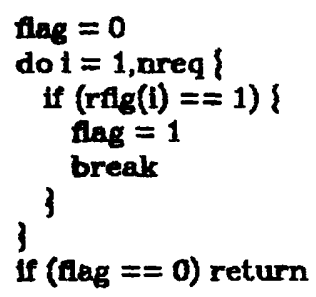

$\#$

GET STATION DATA - IATTTUDE, IONGTTUDE, ELEVATION, INSTRUMENT TYPE

call stadat(Idata, code, lat, lon, elev)

\#

for (:i) $)$

IF MAGRD $==0$, END OF TAPE

if $(\operatorname{magrd}(d r$, nwords, idata, $n r)==0)$ return

IF THE FIRST TWO CHARACTERS OF THE FIRST LINE OF THE BUFFER BQUAIS 'ST', THIS IS A STATION IOG

call getlin(idata, 1 , ibuff)

if (ibuff(1) $==$ kus \& ibuff $(2)==$ kut) break

IF THIS WAS NOT A STATION LOG, IT IS A DATA LOG.

GET DATA FROY FIRST DATA LOG RECORD -

AVE CAIIBRATTON VALUES FOR EACH CHANNEL.

If (Getcal(Idata, Ichan, cal,linstr) $==0)$ \} write $(6,13)$

13 3

format (1x, 'Error reading - calibration')

CHECK TO SEE OF THE USER HAS REQUESTED INFORMATTON TO BE EXTRACTED FROM THIS STATTON AND INSTRUMENT 


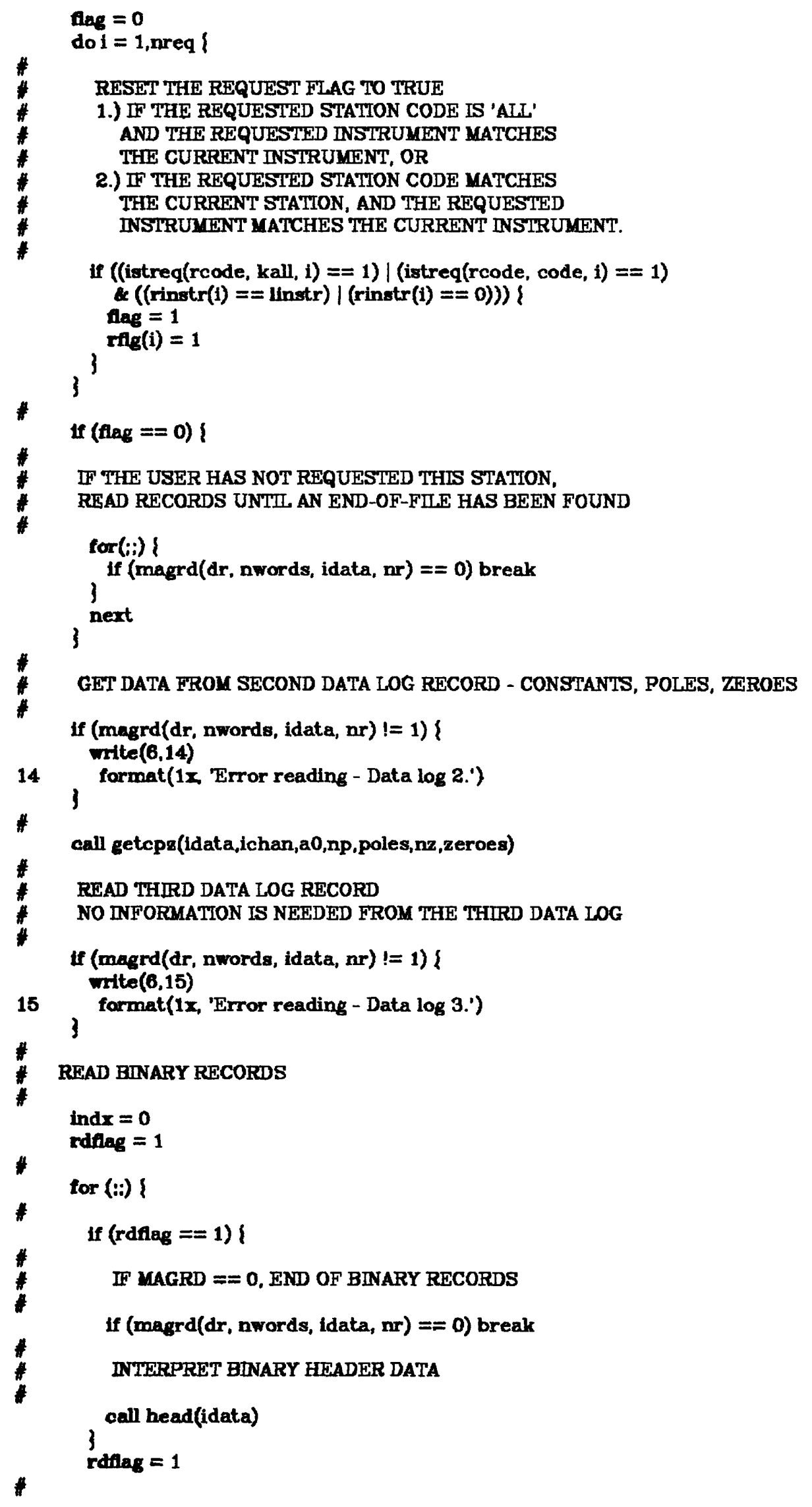




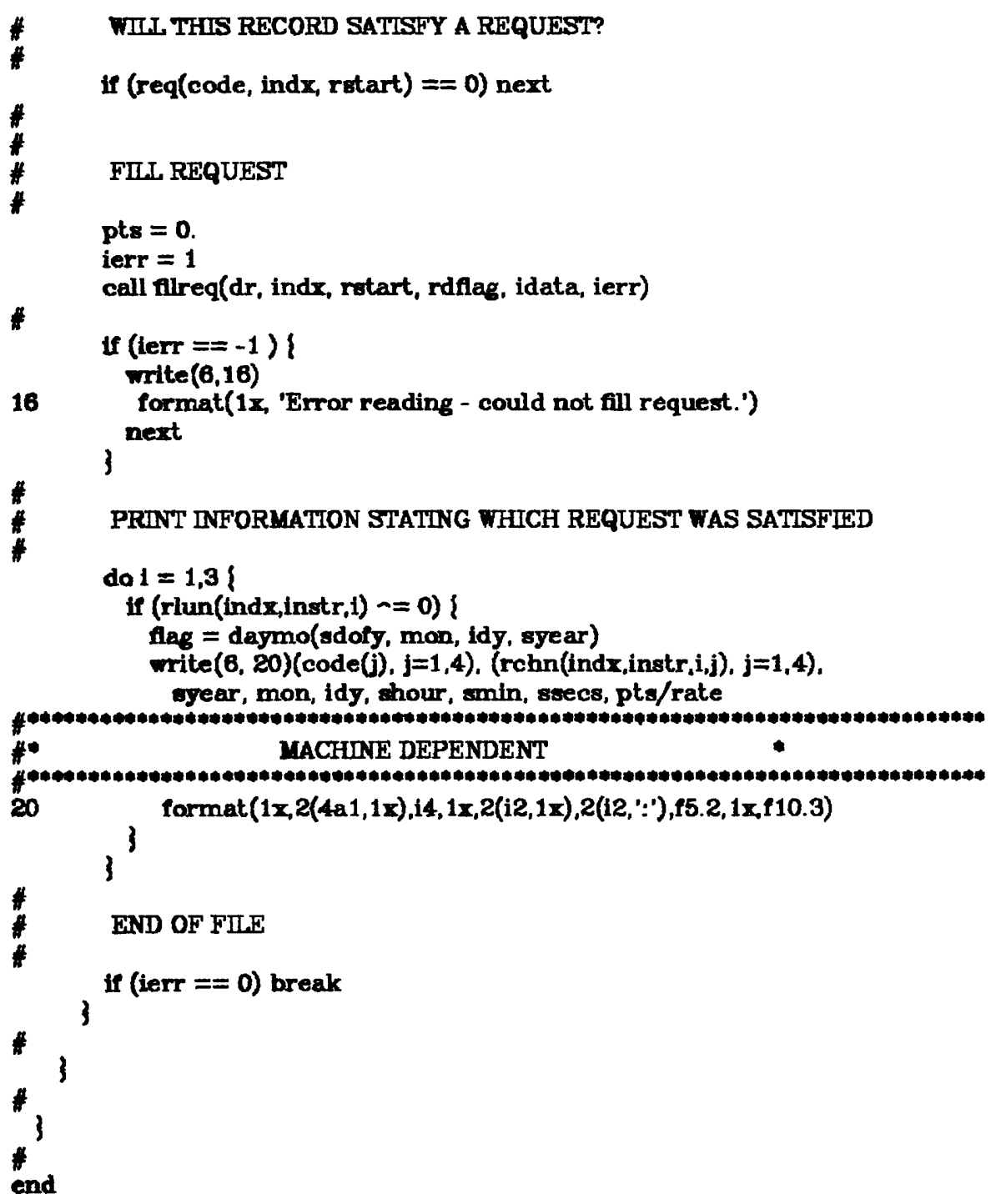


RDNTLG.RATFOR

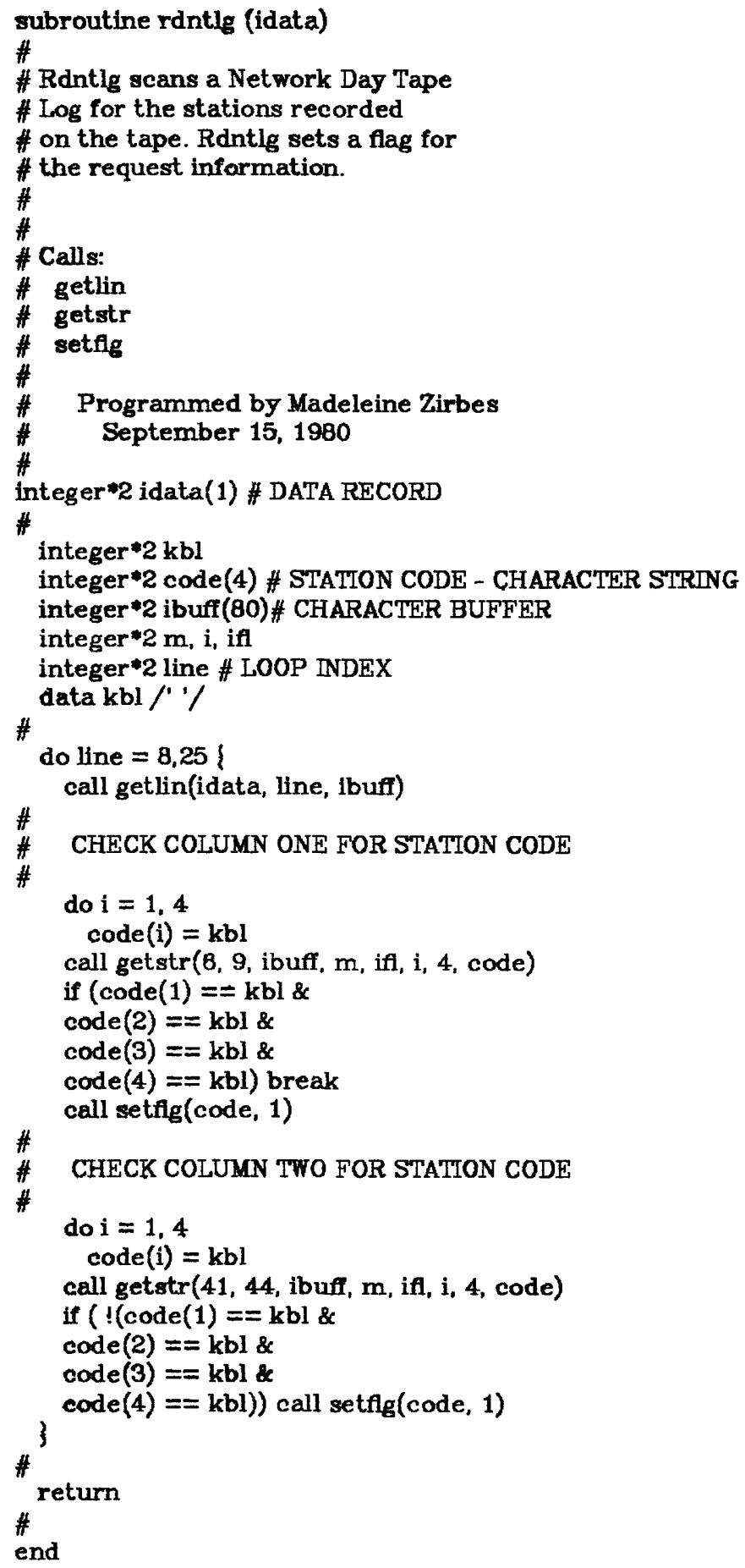




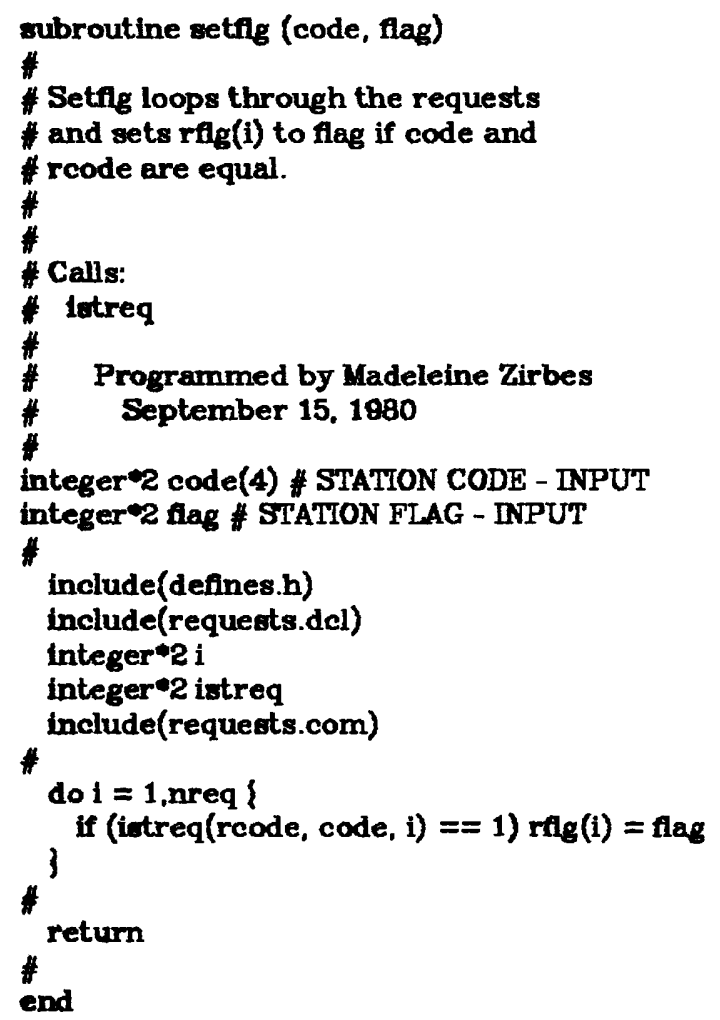




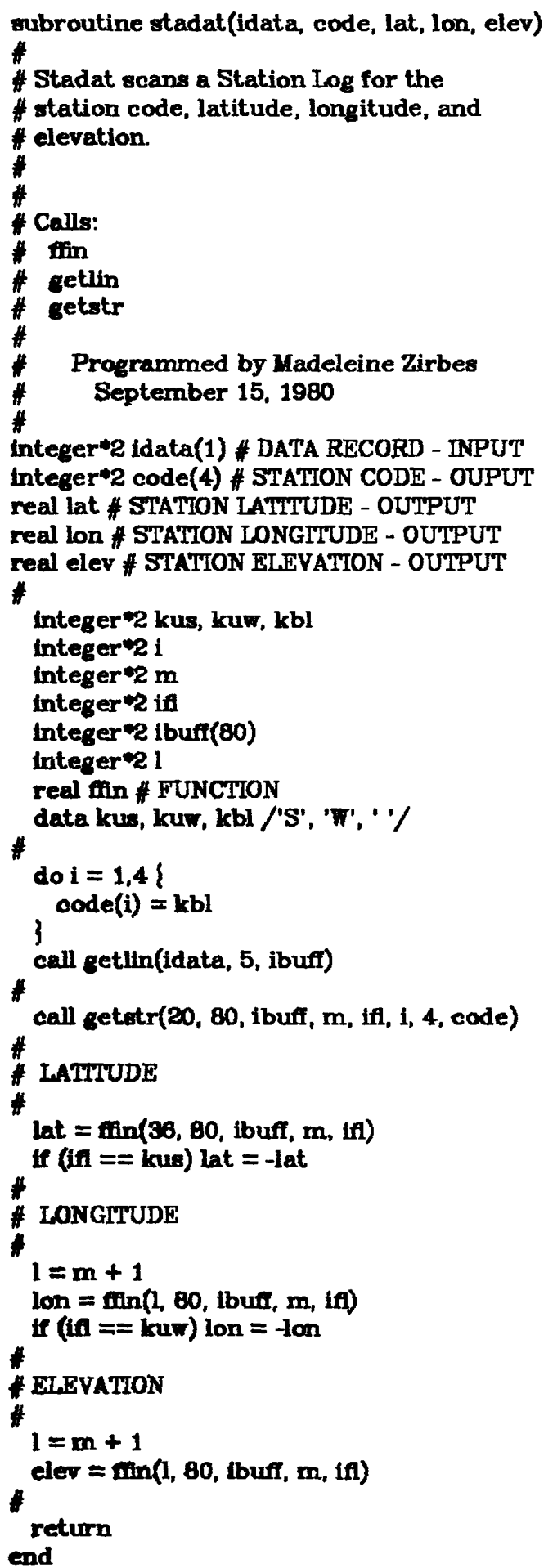




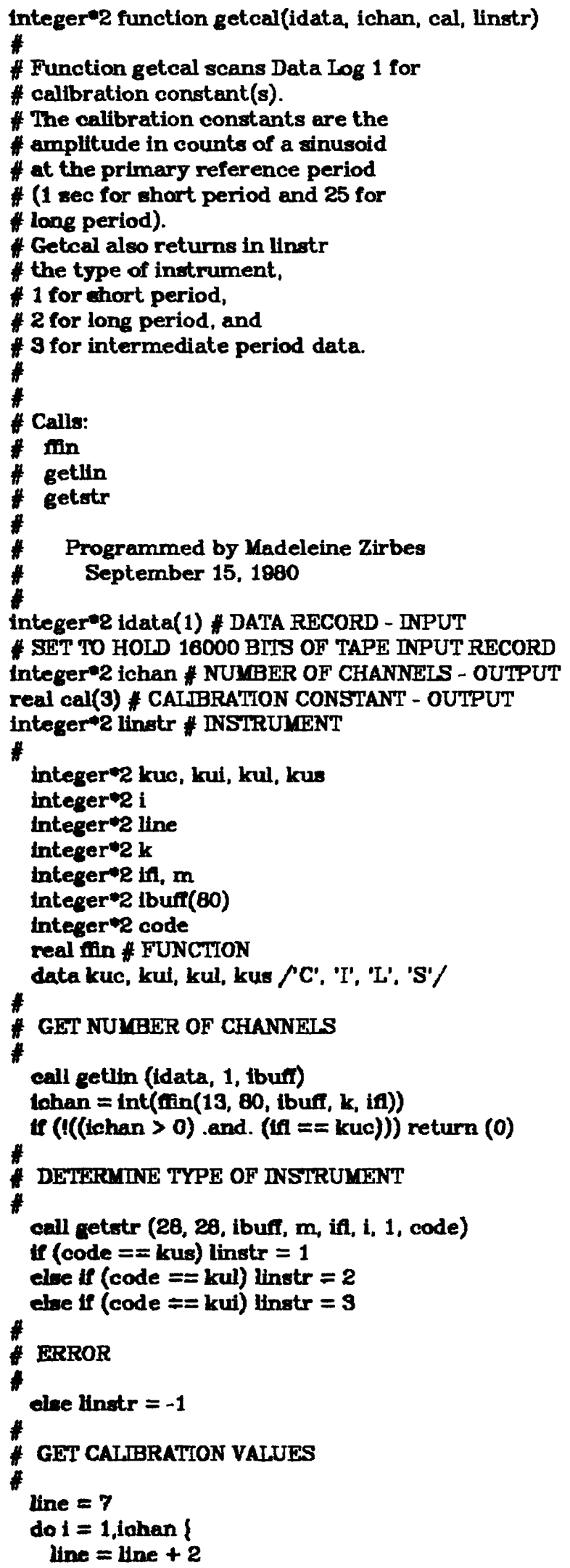


call getlin (idata, line, ibuff)

$\operatorname{cal}(1)=\operatorname{fin}(51,80,1$ buff, $k$, if $)$

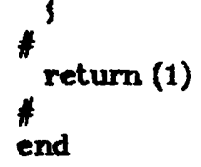

end 


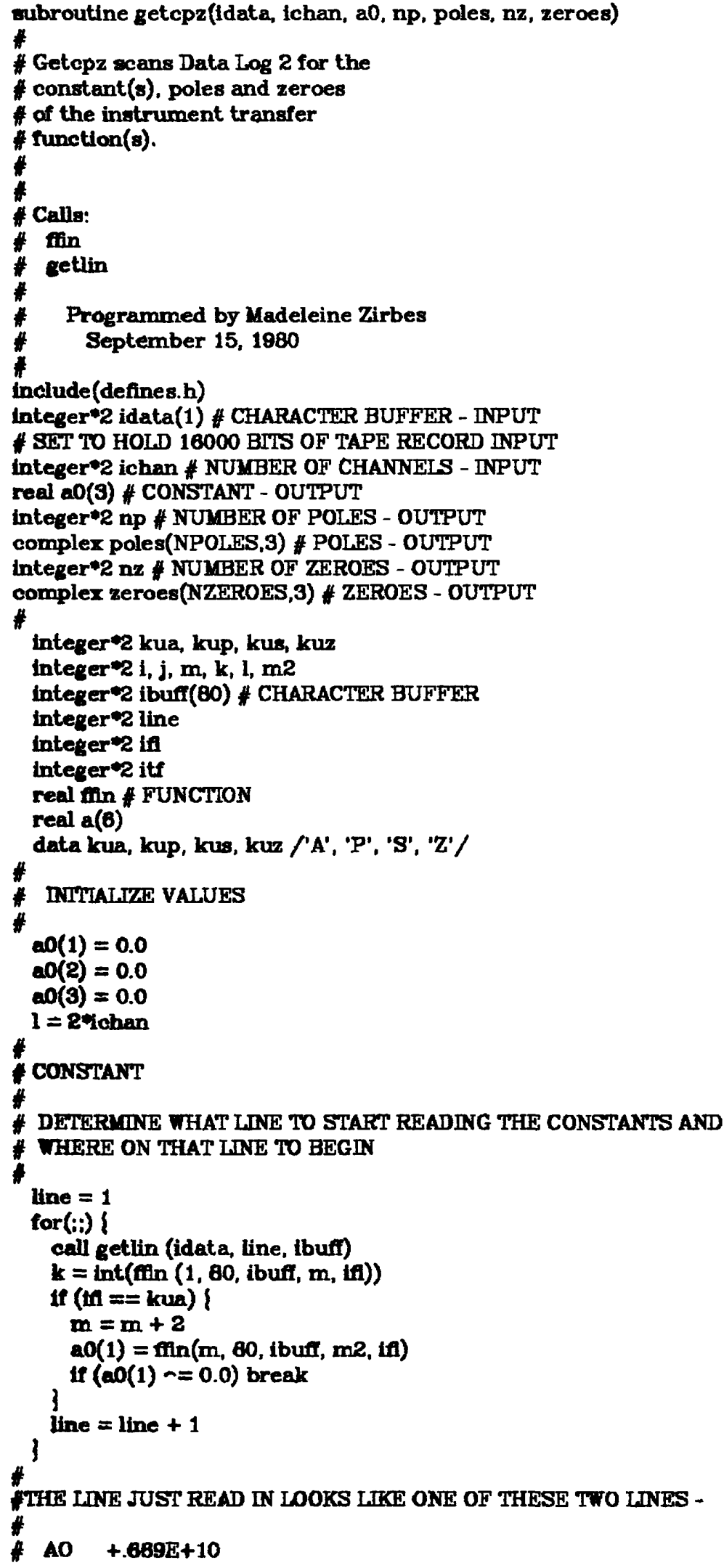




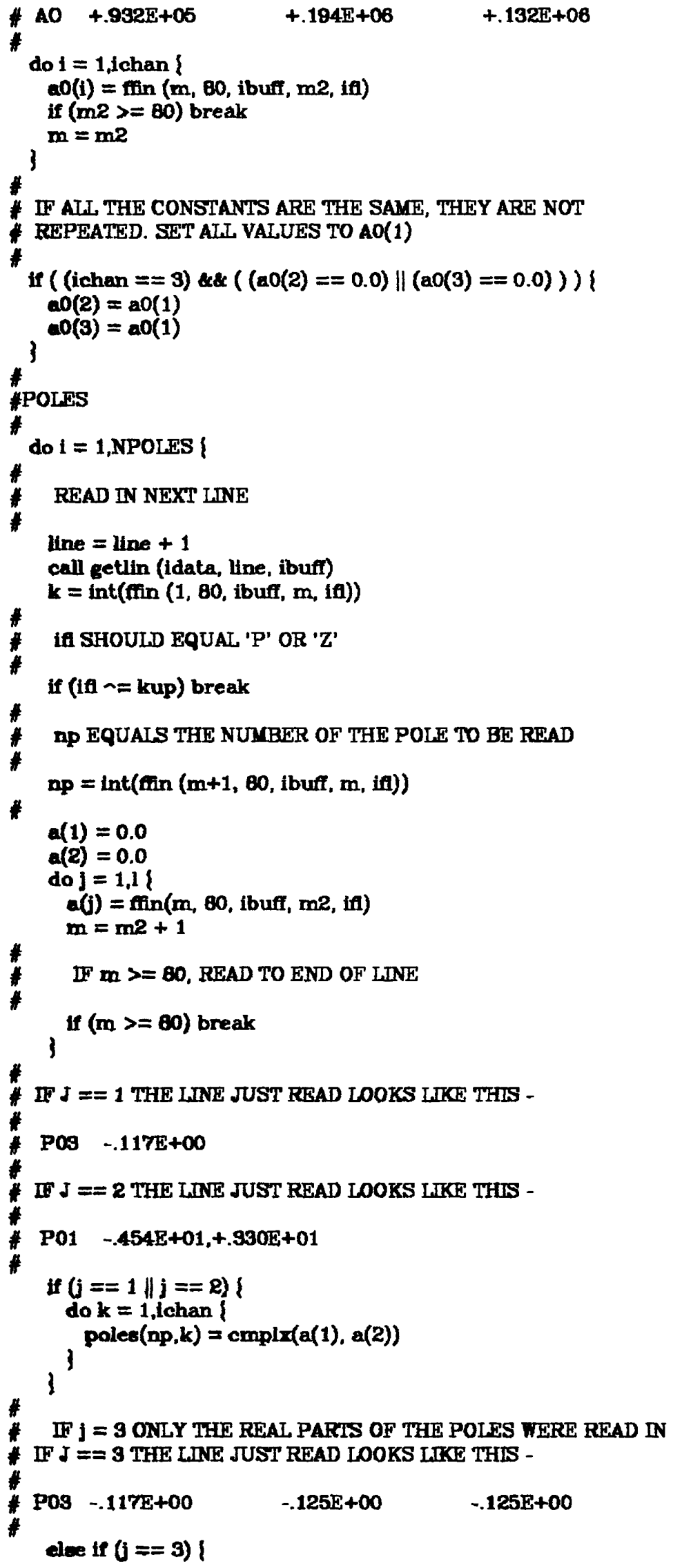




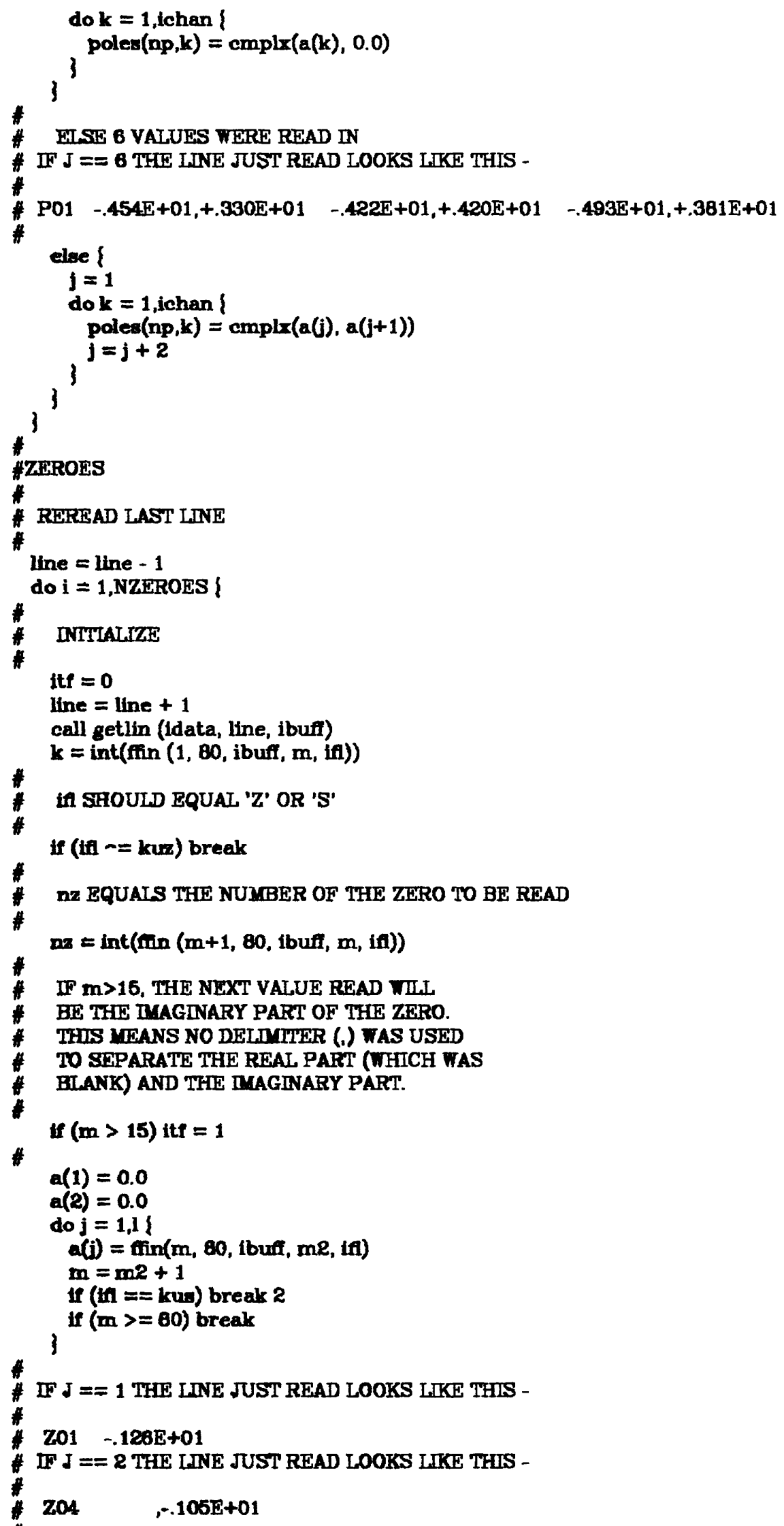




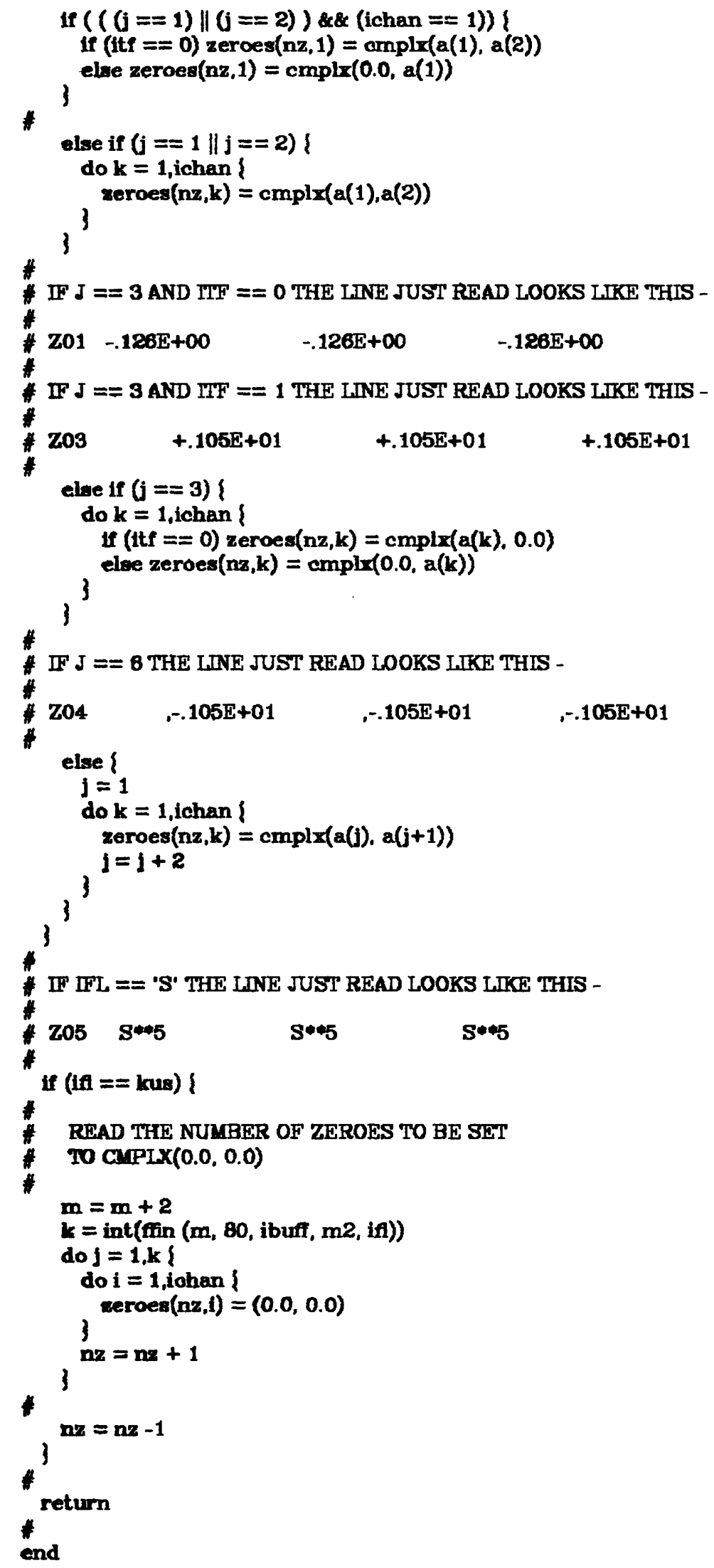


REQ.RATFOR

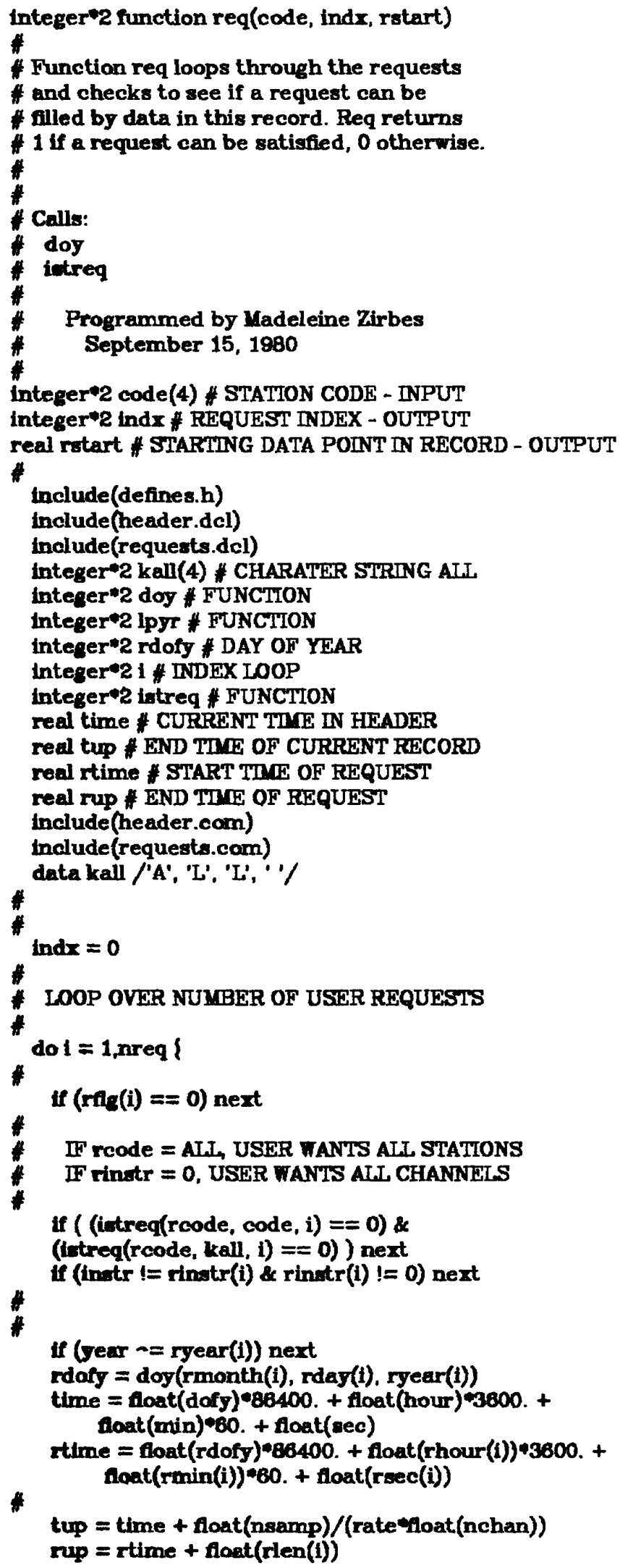




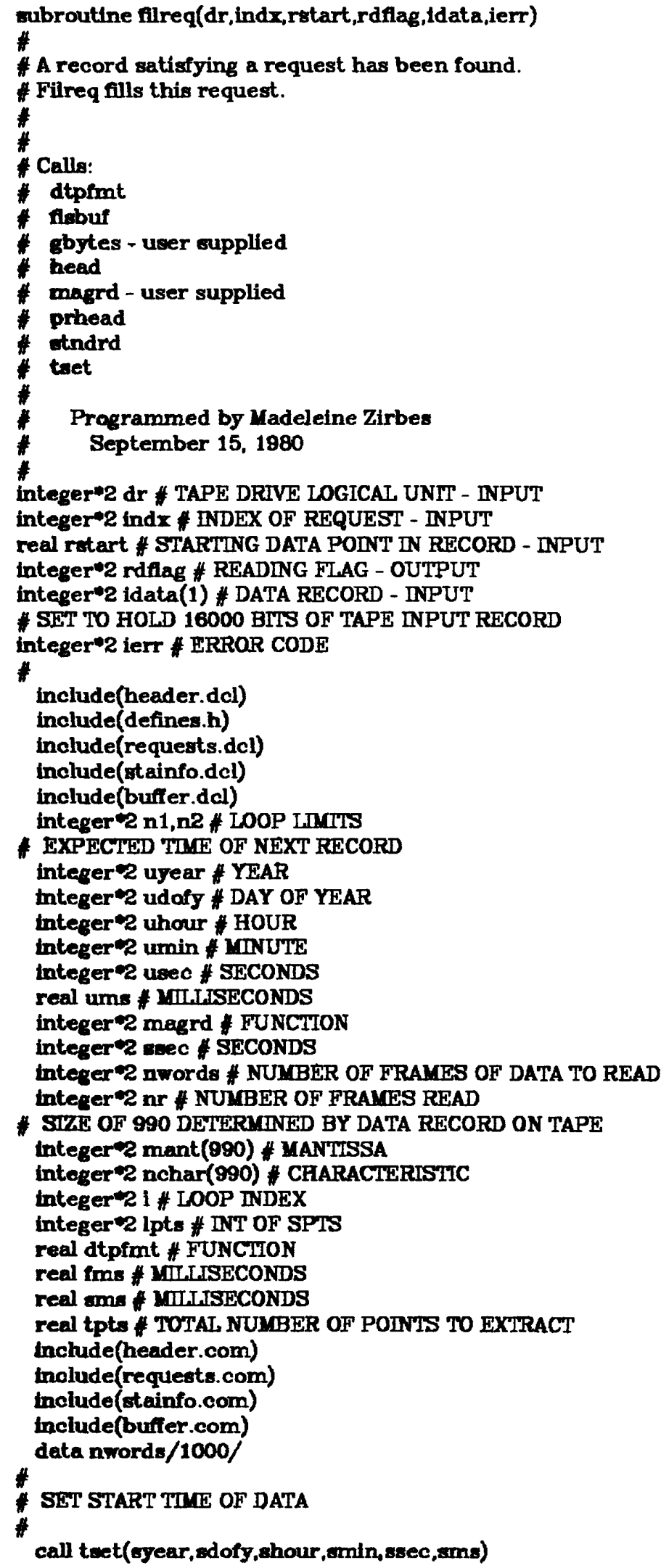




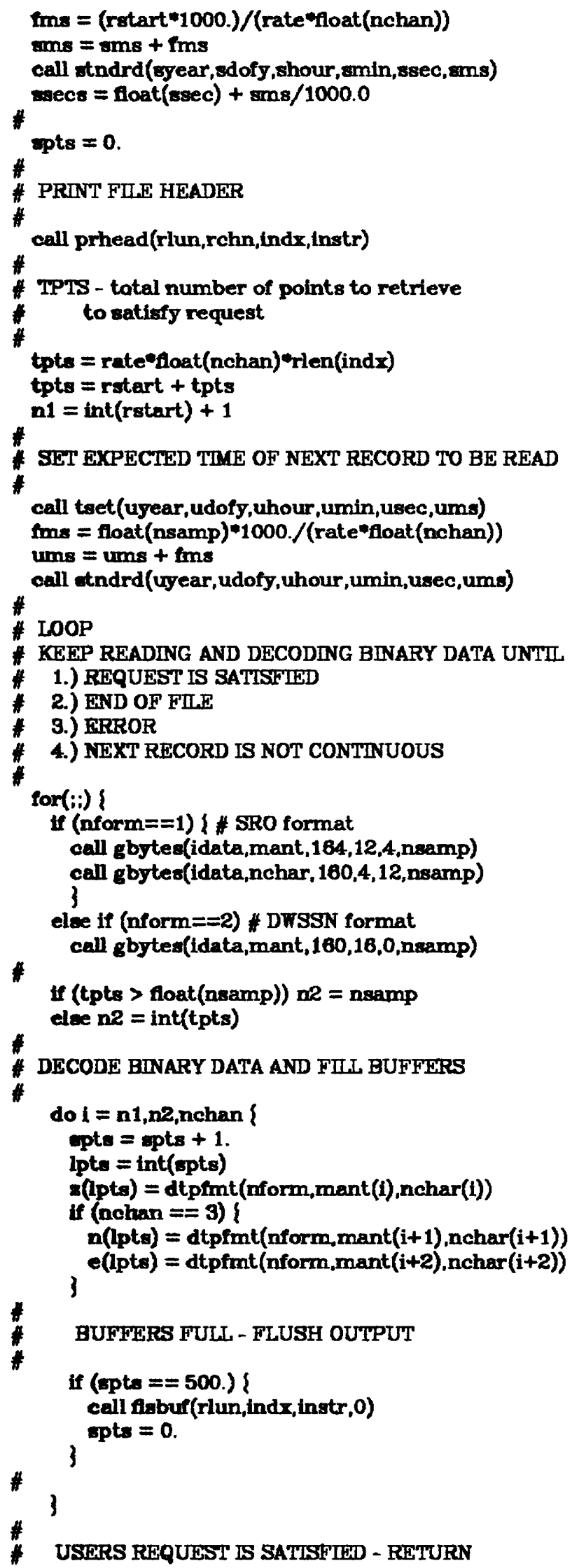




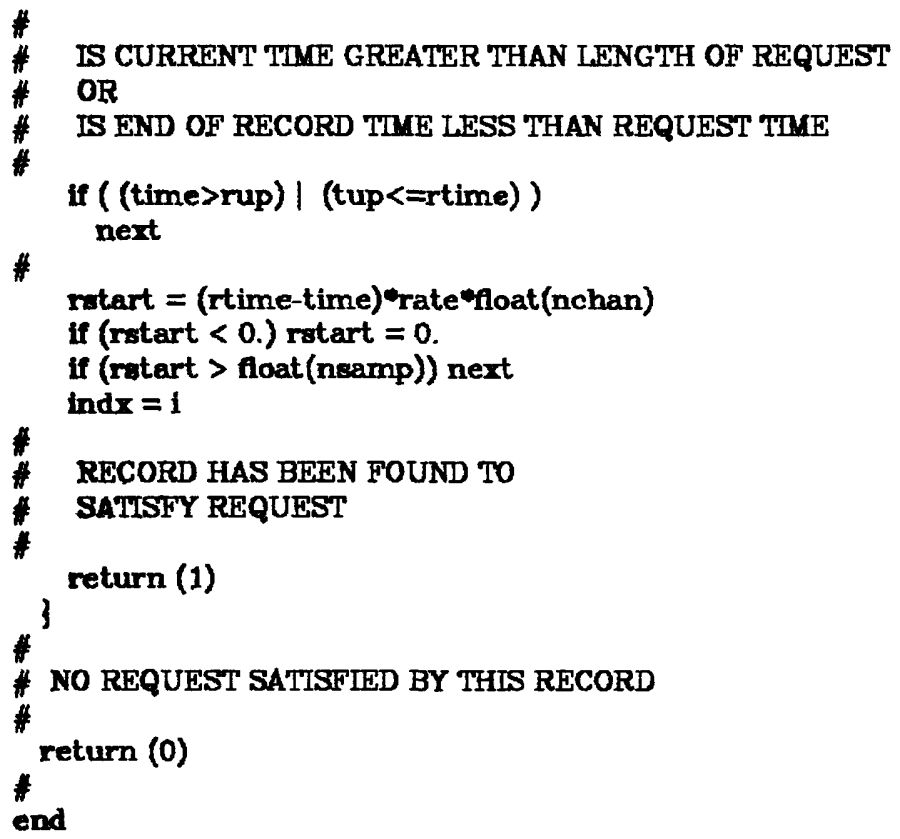




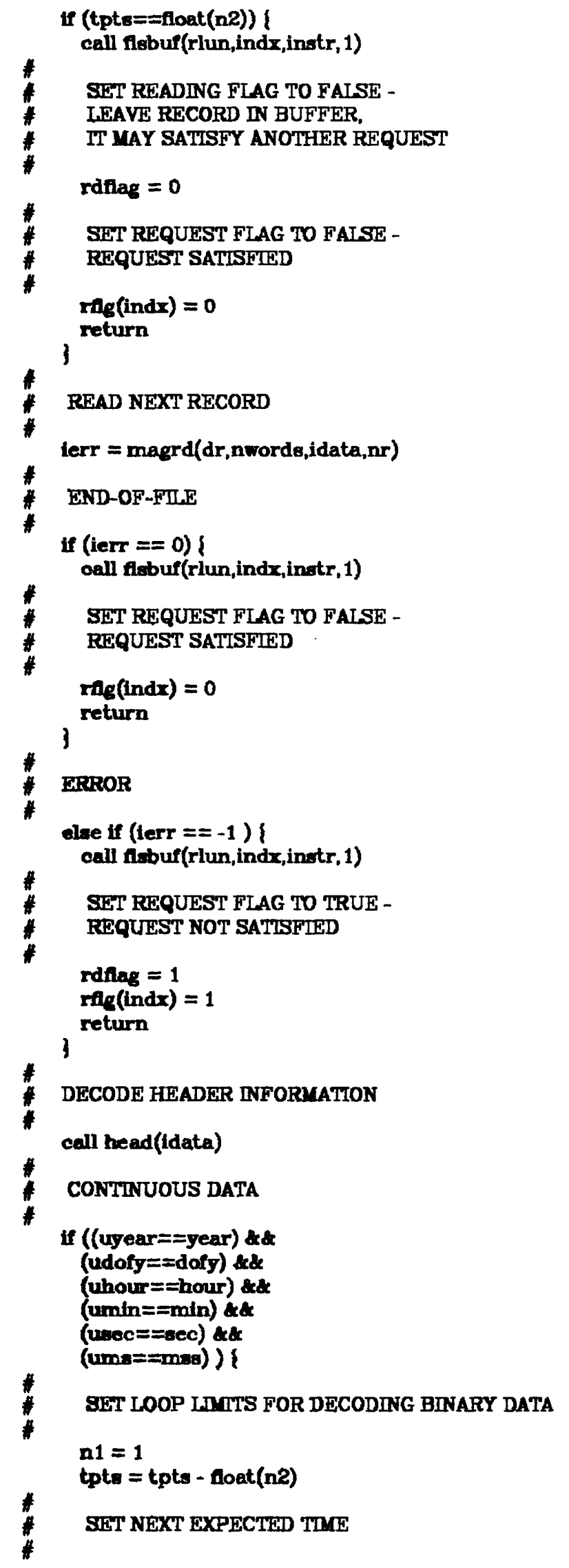




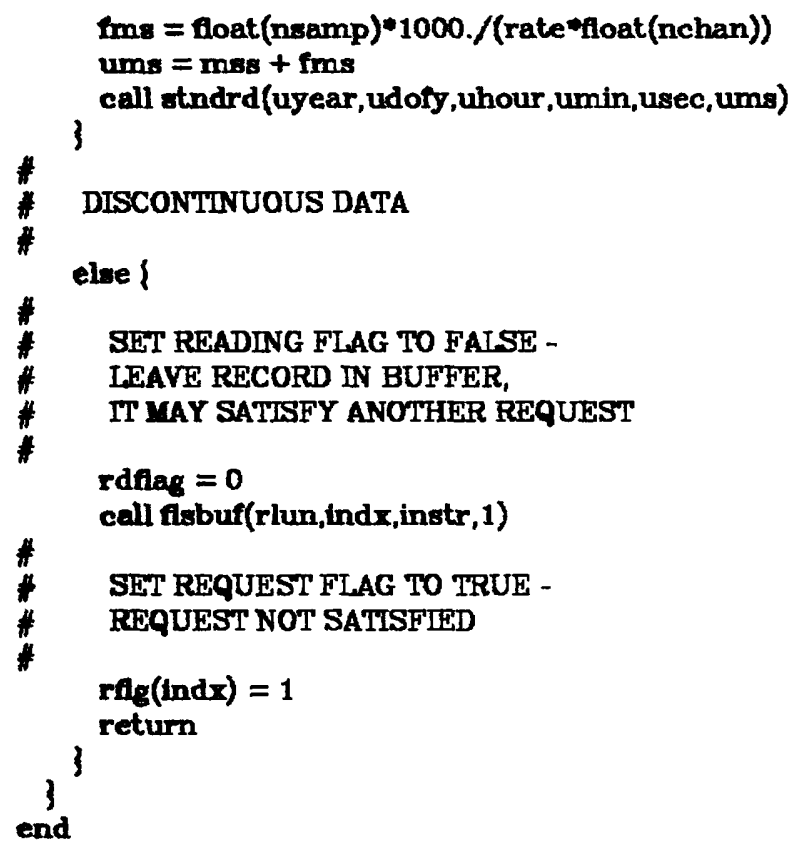


PRHEAD.RATFOR

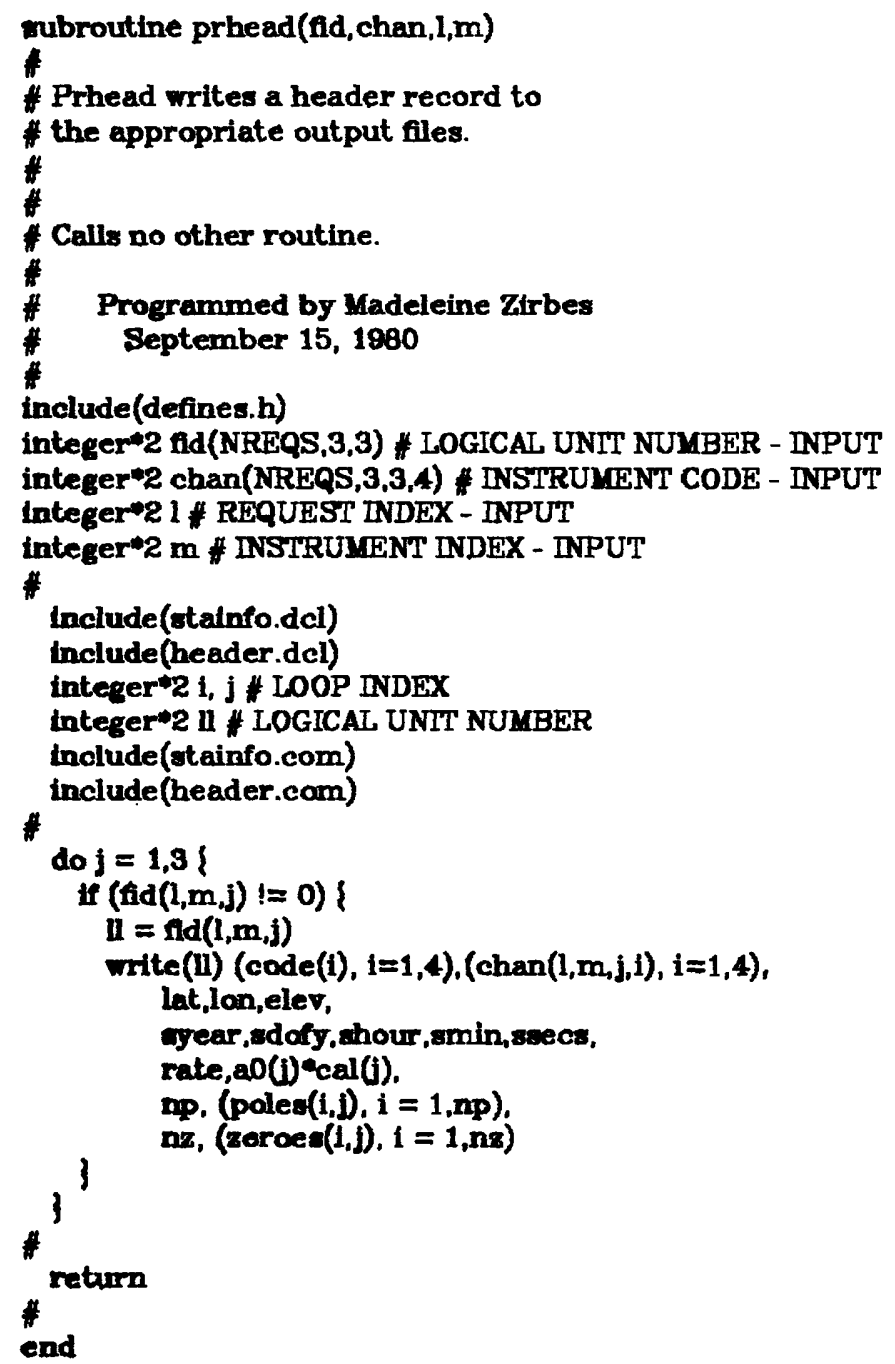




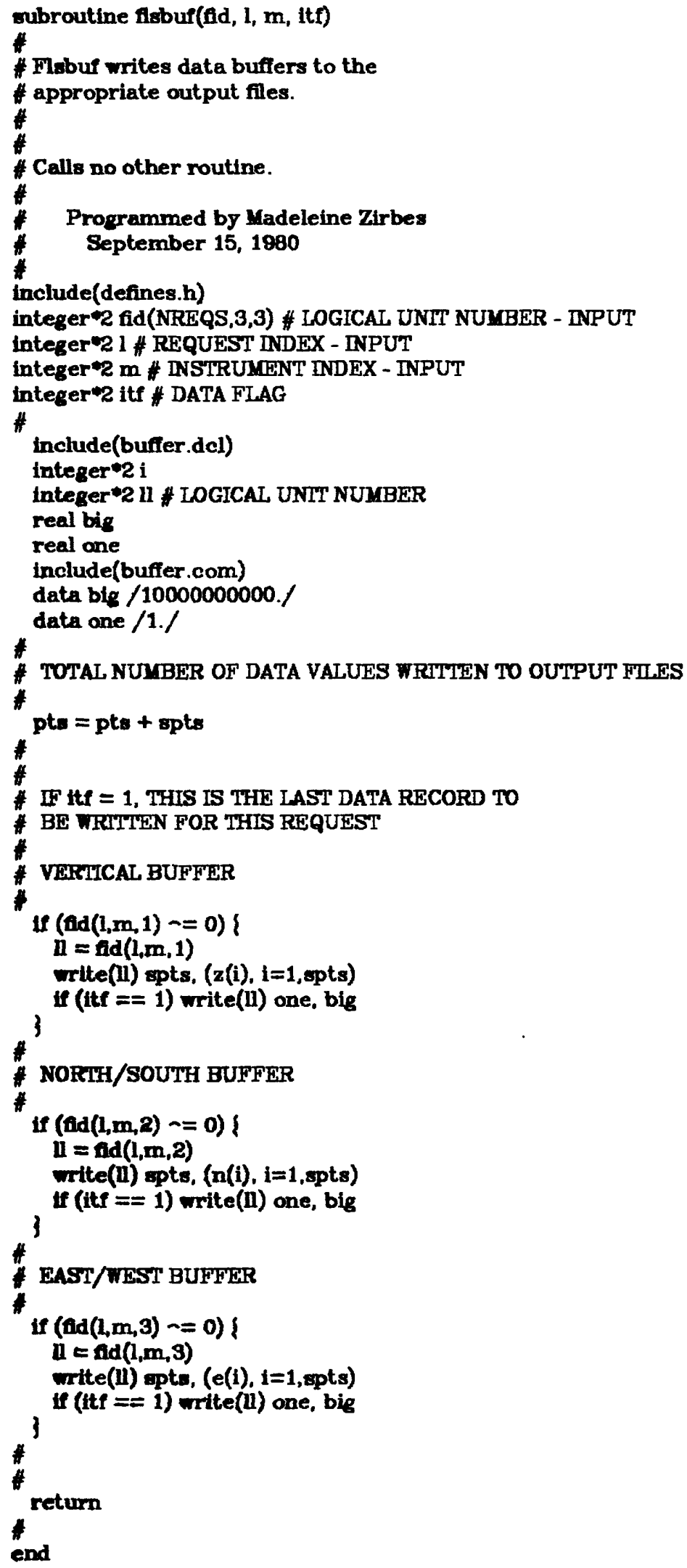


TIIE' HANDLING ROUTINES

TSET.RATFOR

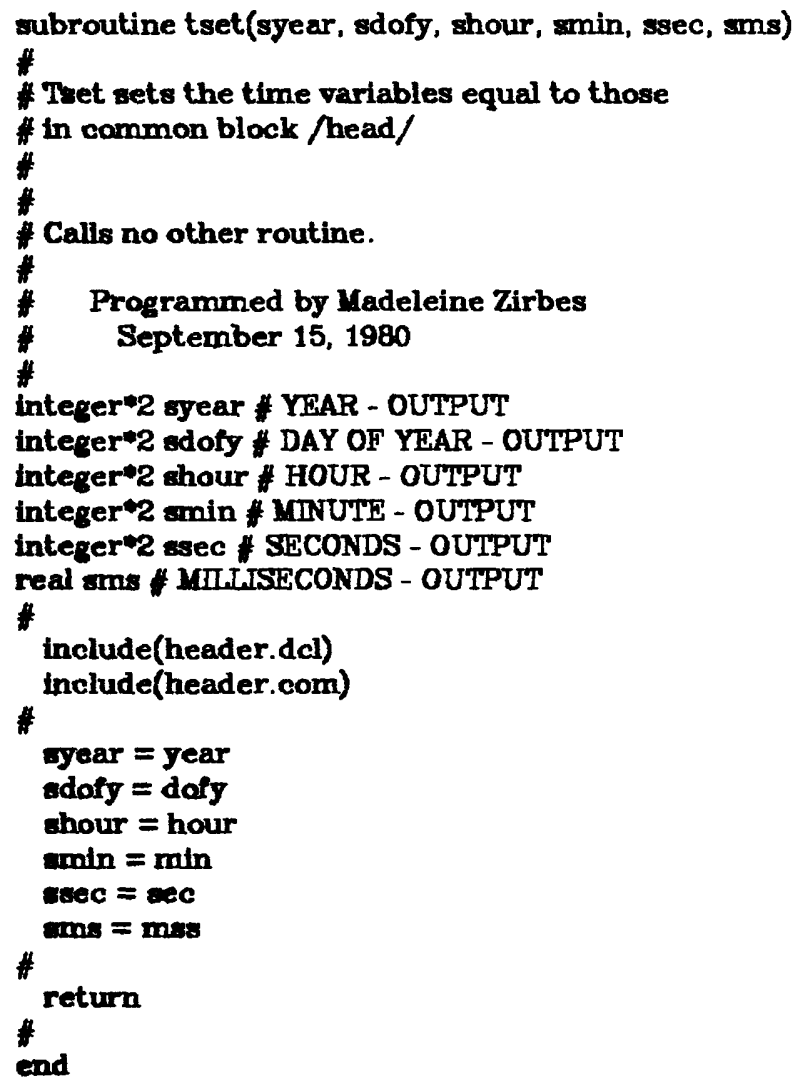




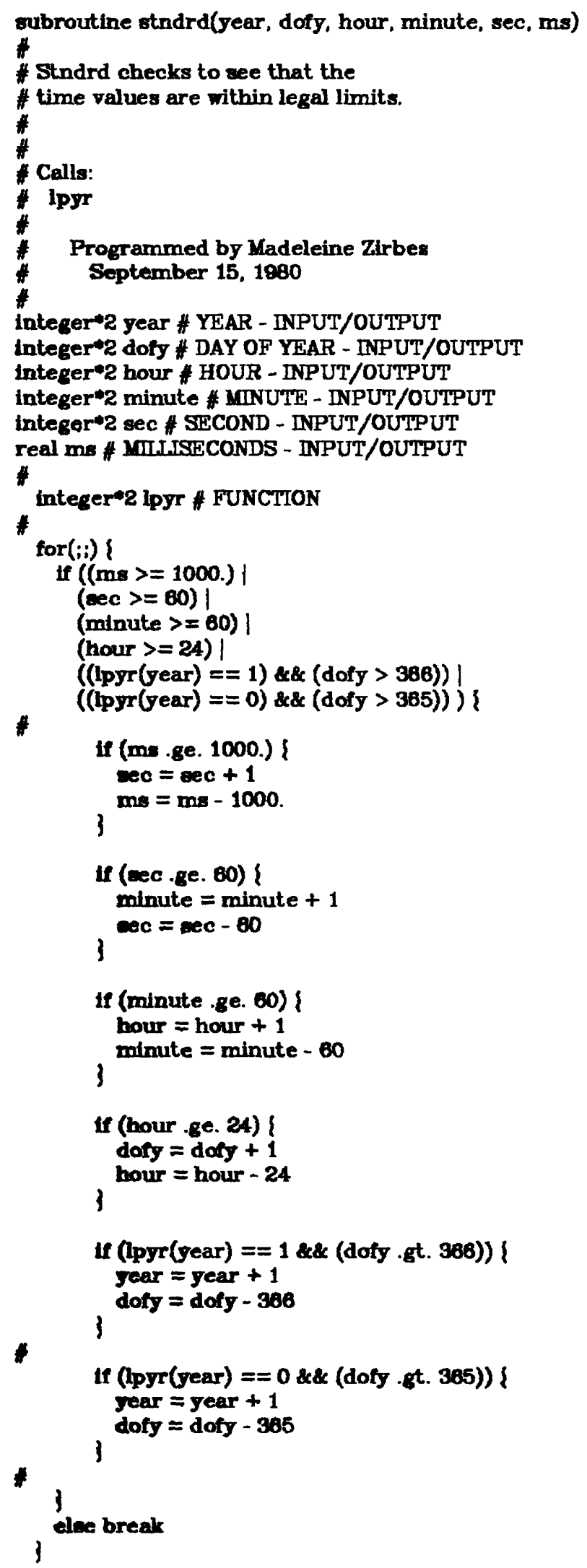


$-78-$

return

$\#$
end 


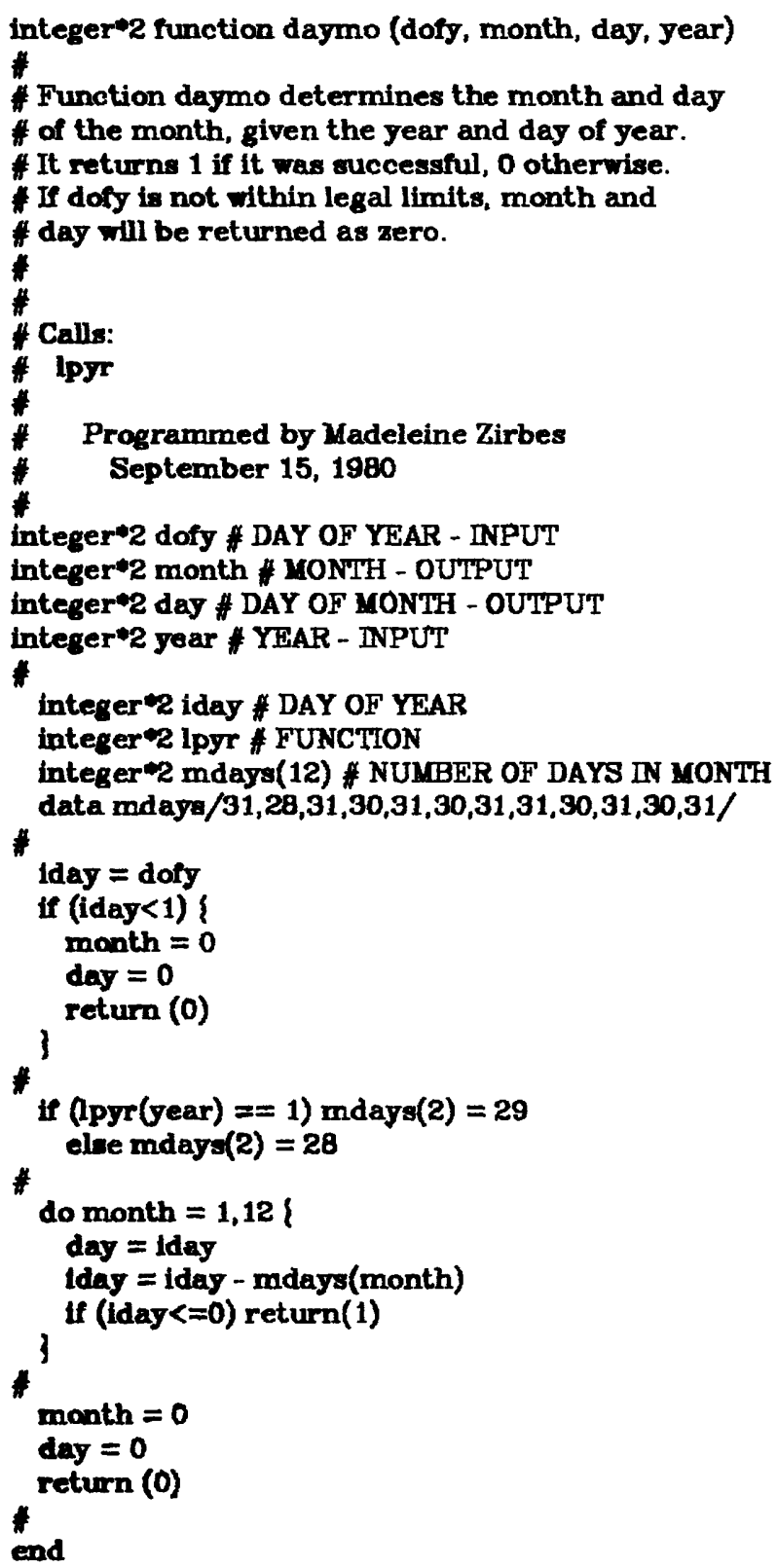


DOY.RATFOR

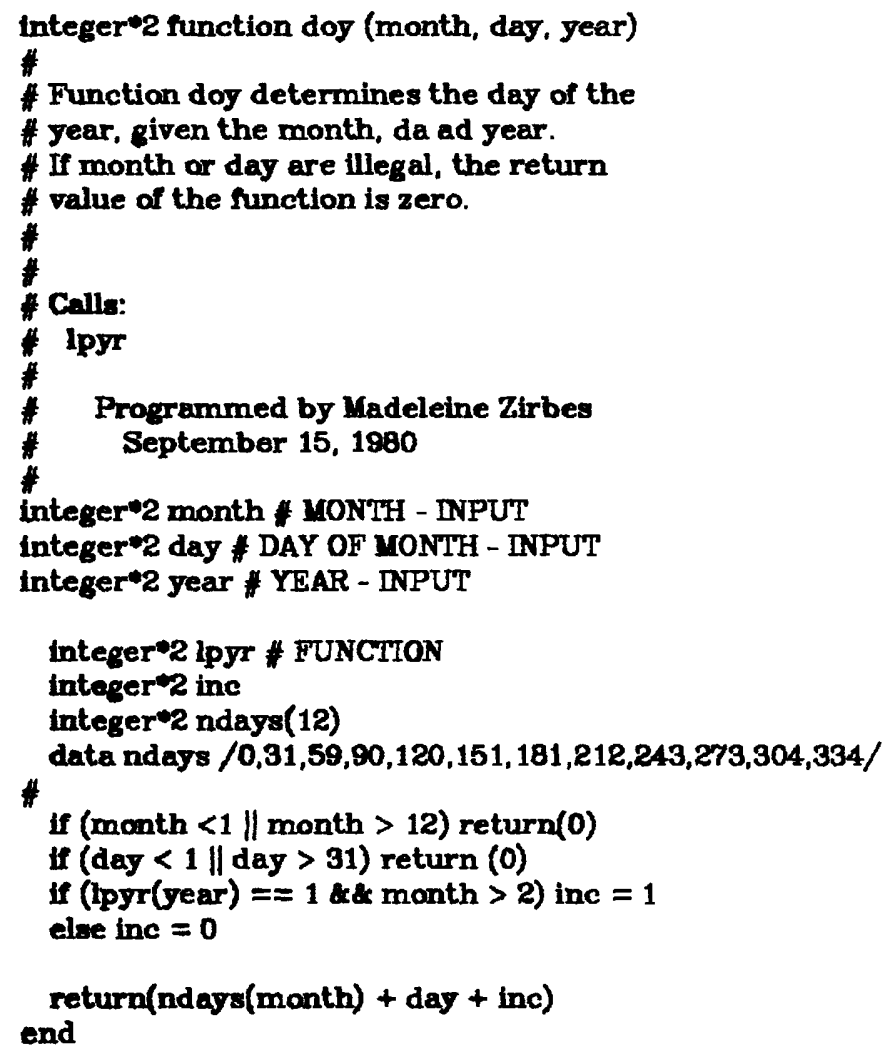


IPYR.RATFOR

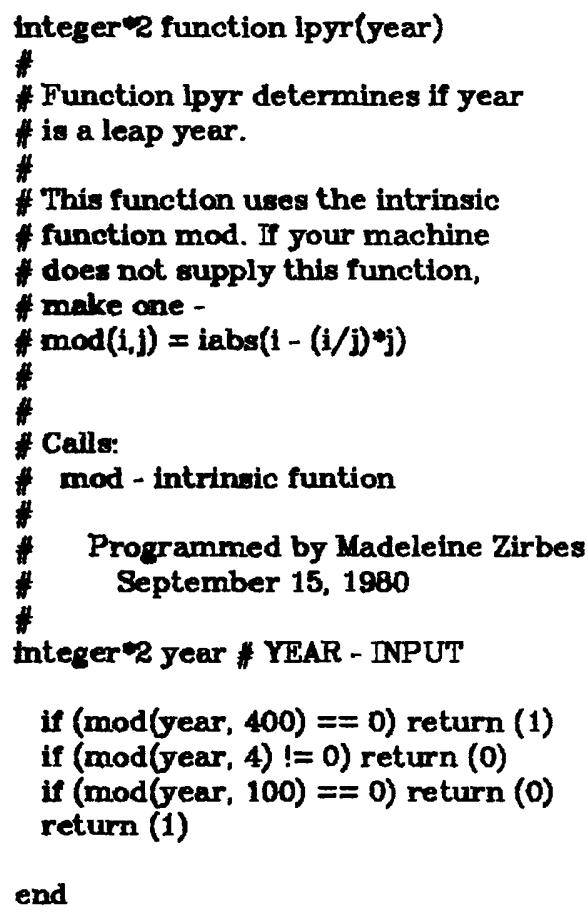




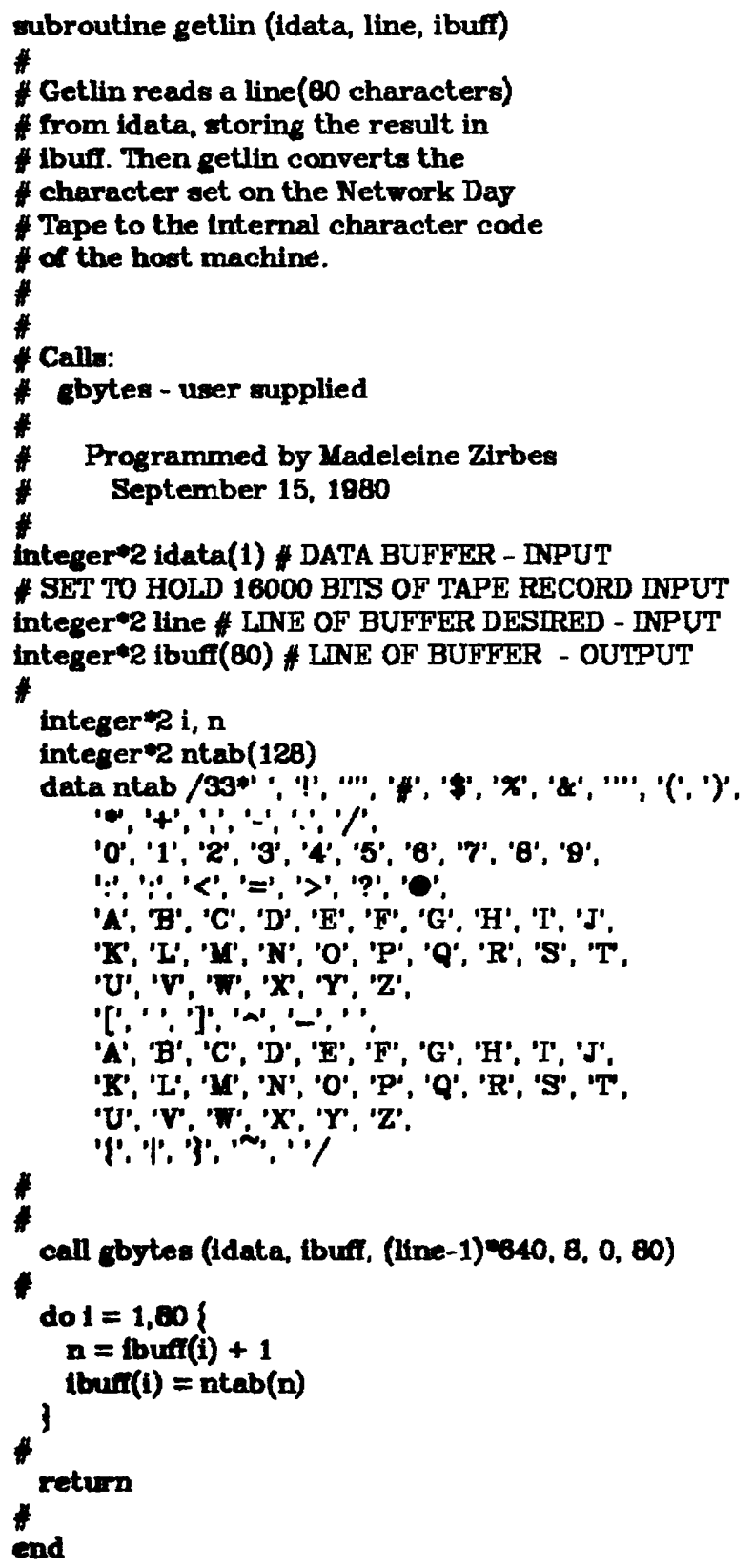


FTIN.RATFOR

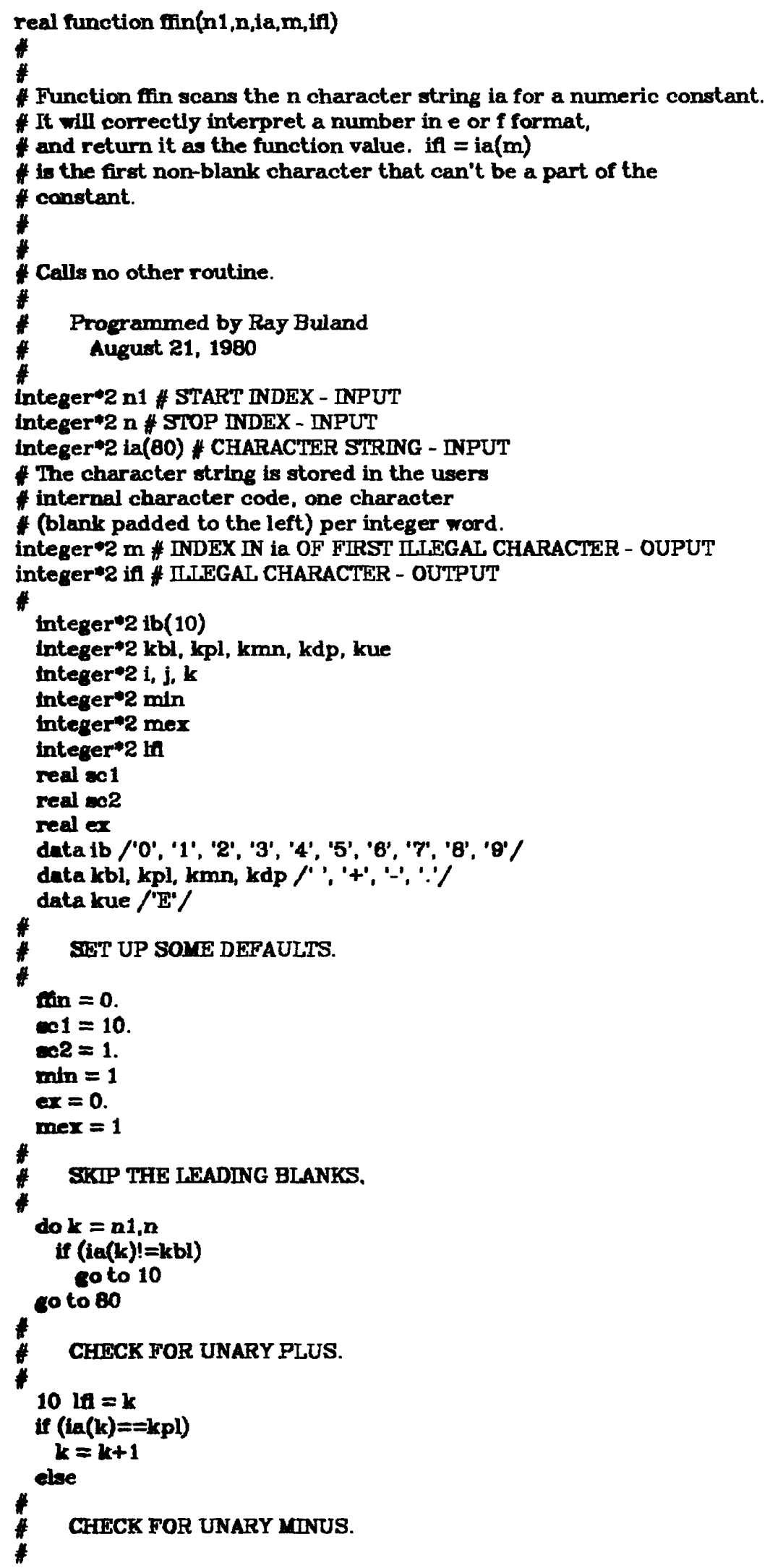




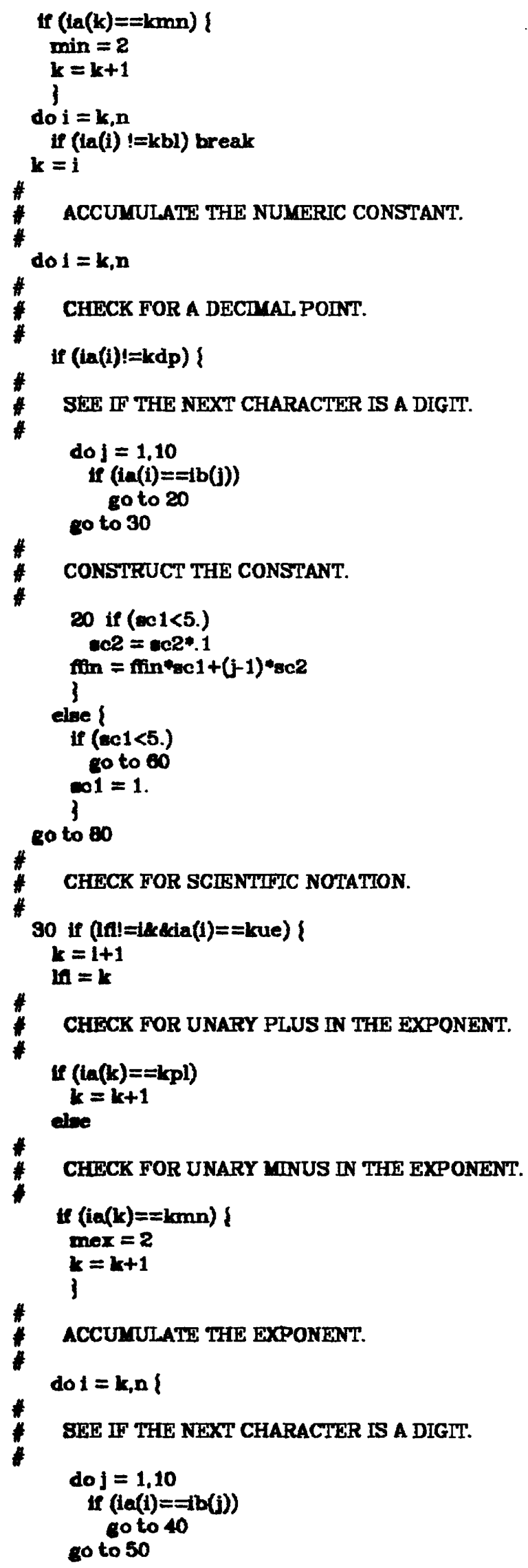




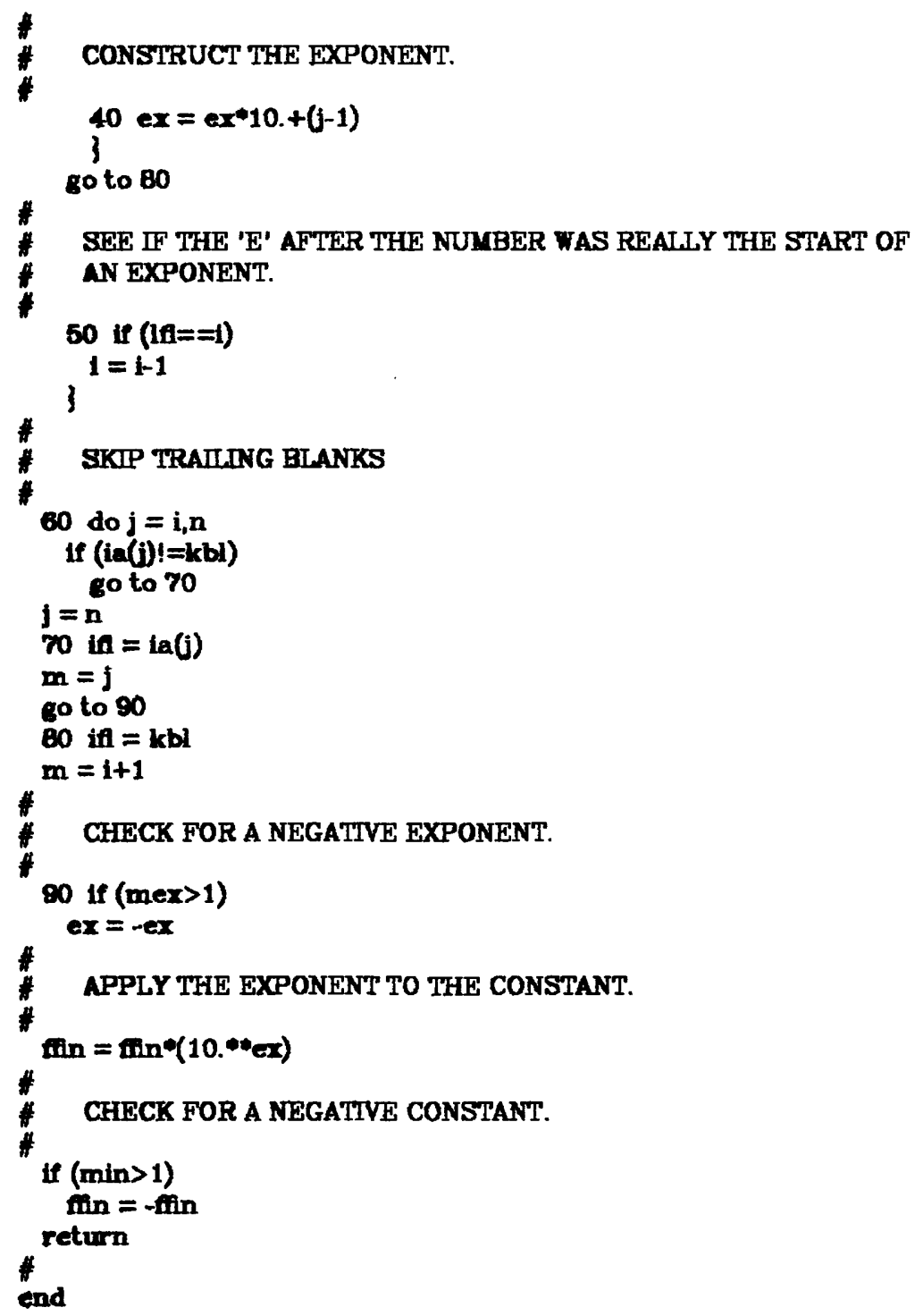




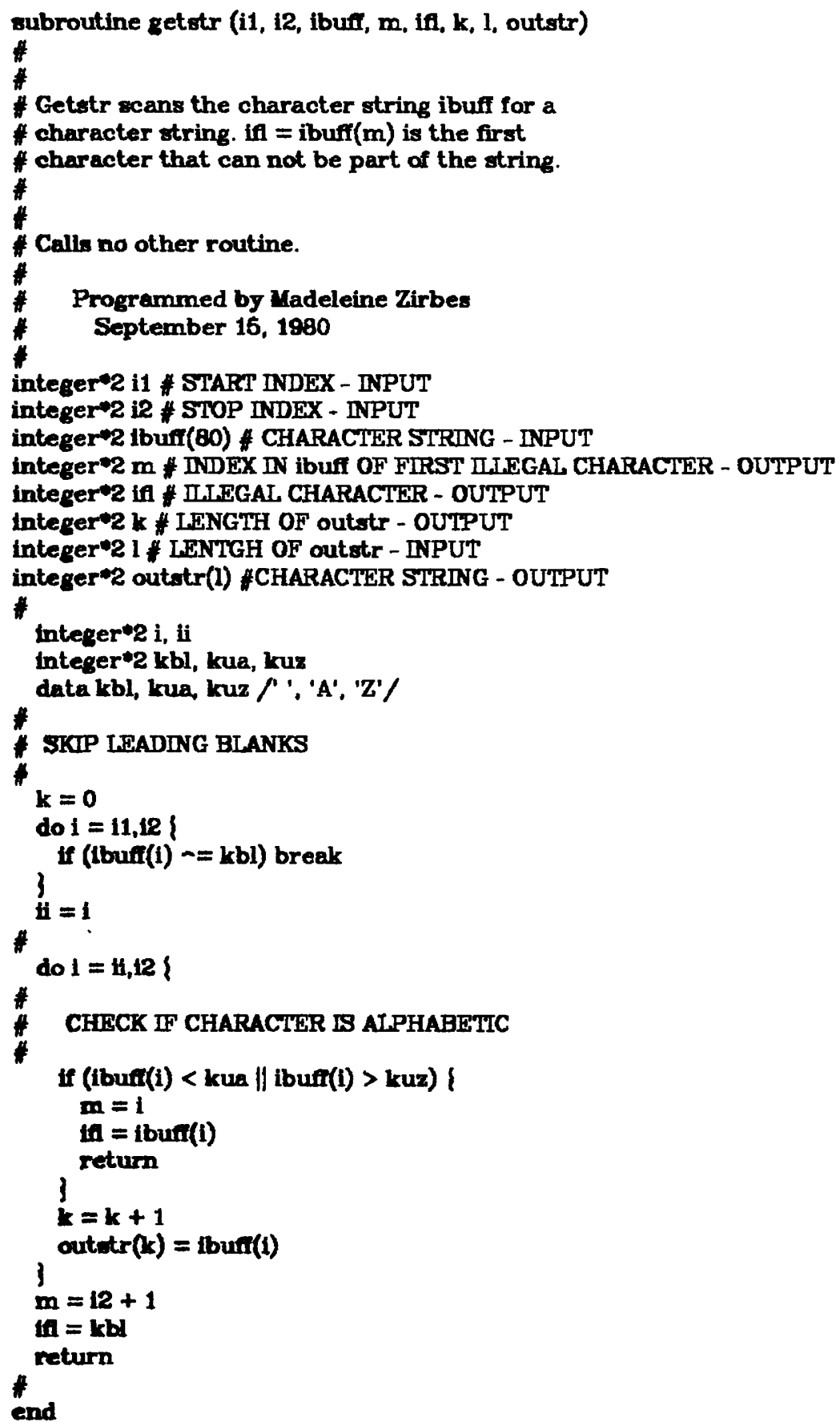




\section{ICPYCH.RATFOR}

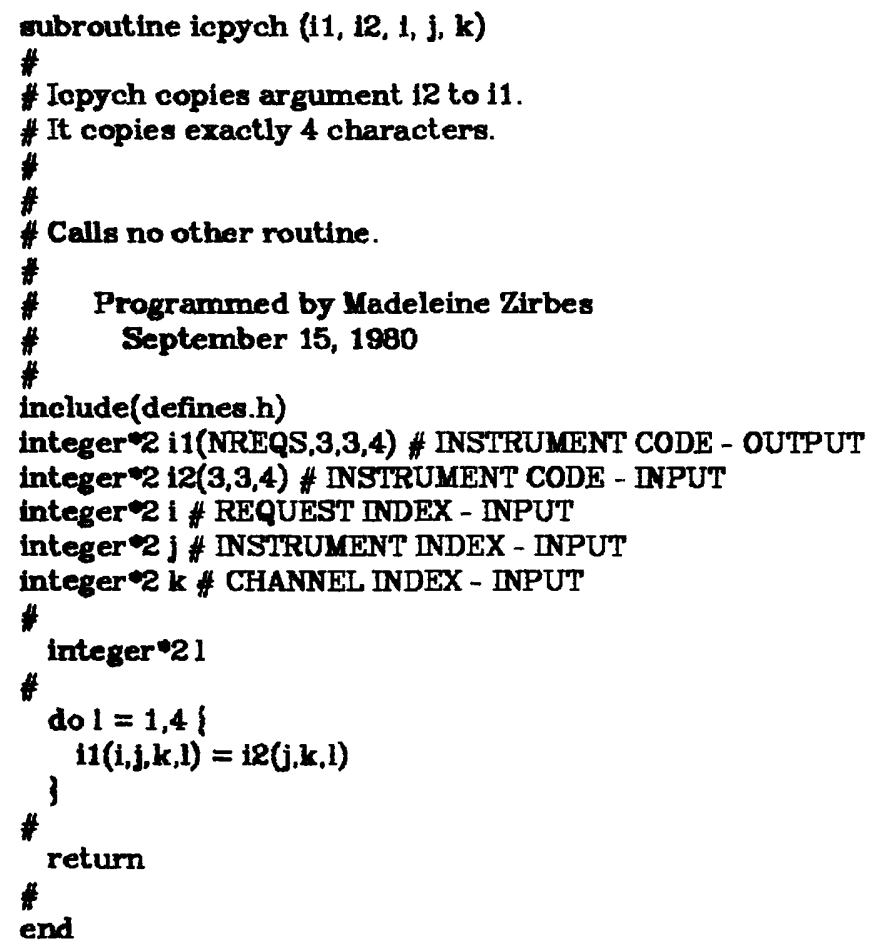


ISTREQ.RATFOR

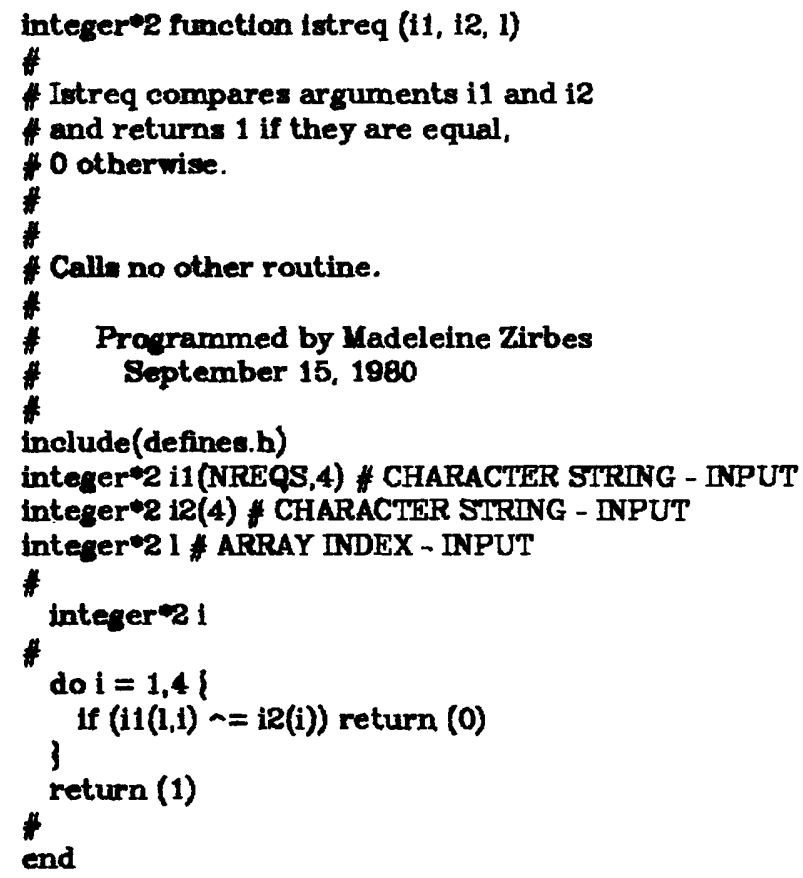


BINARY DATA HANDIRNG ROUTINES

HEAD.RATFOR

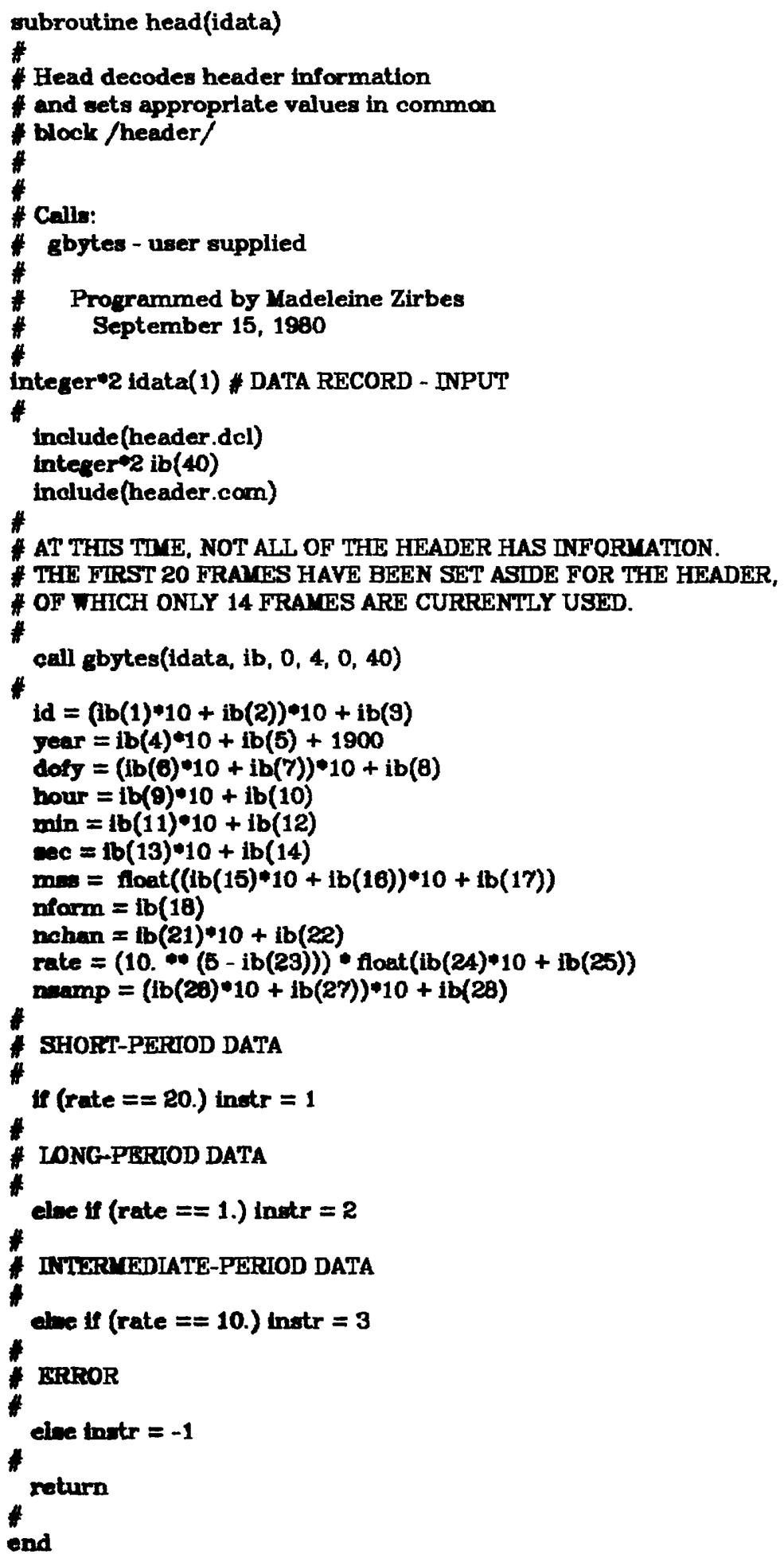


real function dtpimt(nform, mant, char)

*⿻

* Function dtpfmt decodes one data word from

\# a Network Day Tape, and returns it as

f the function value.

* If nform = 1, the data is in SRO format;

* if nform = 2, the data is in DWWSSN format.

it

* SRO and ASRO data samples are recorded as

* 16 bit ( 2 frames) words using a gain-range

- format. The four most sienificant bits of

\# frame 1 define the gain factor(GF) which is

* an unalgned integer having a value from

* 0 to 10. The following bit defines the aign

* of the mantisea, and the 3 remaining bits

\# plus the $\mathbf{B}$ bits of frame 2 contain seismic

* data in twos complement binary integer form.

4 Abeohute amplitude in digital counts is

* derived as follows -

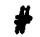

* Amplitude $=$ Mantissa $* 2 *(10-\mathrm{GF})$

A

\# This gives a dynamic range of $72 \mathrm{db}$, an

* operating range of $120 \mathrm{db}$ and a maximum

amplttude of $+2,090,128$ to $-2,087,152$

* digital counts.

*

- DWWSSN data samples are recorded in a

* 16-btt tros complement integer format. This

* gives a dynamic range of $88 \mathrm{db}$ and

f a maximum amplitude of $+32,767$ to

* -32,708 digital counta.

i.

\# Dtpimt assumes that the users machine has

\# a word length of at least 16 bits.

it

* Frame is defined as 8 contiguous byte

* allened bits by the tape drive.

it

H

* Calle no other routine.

.

* Programmed by Madeleine Zirbes

- September 15, 1990

4

integer 4 nform * FORIAT ID - INPUT

interer 0 mant MANTISSA - INPUT

integer char CHARACTERISTIC INPUT

it

real pwr(16)

data pwr/1024., 512., 256., 128., 64., 32., 16., 8., 4., 2., 1.,

$.5, .20, .125, .0625, .03125 /$

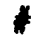

SRo Format

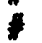

If (nform $==1)$ (

if (mant.ge.2048) mant $=$ mant -4008

dipint = foat $(\operatorname{man} t)$ pwr $($ char +1$)$

$4^{3}$

return

DWISEN FORYAT 


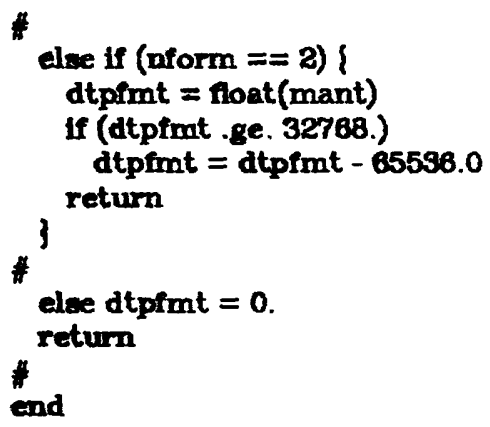




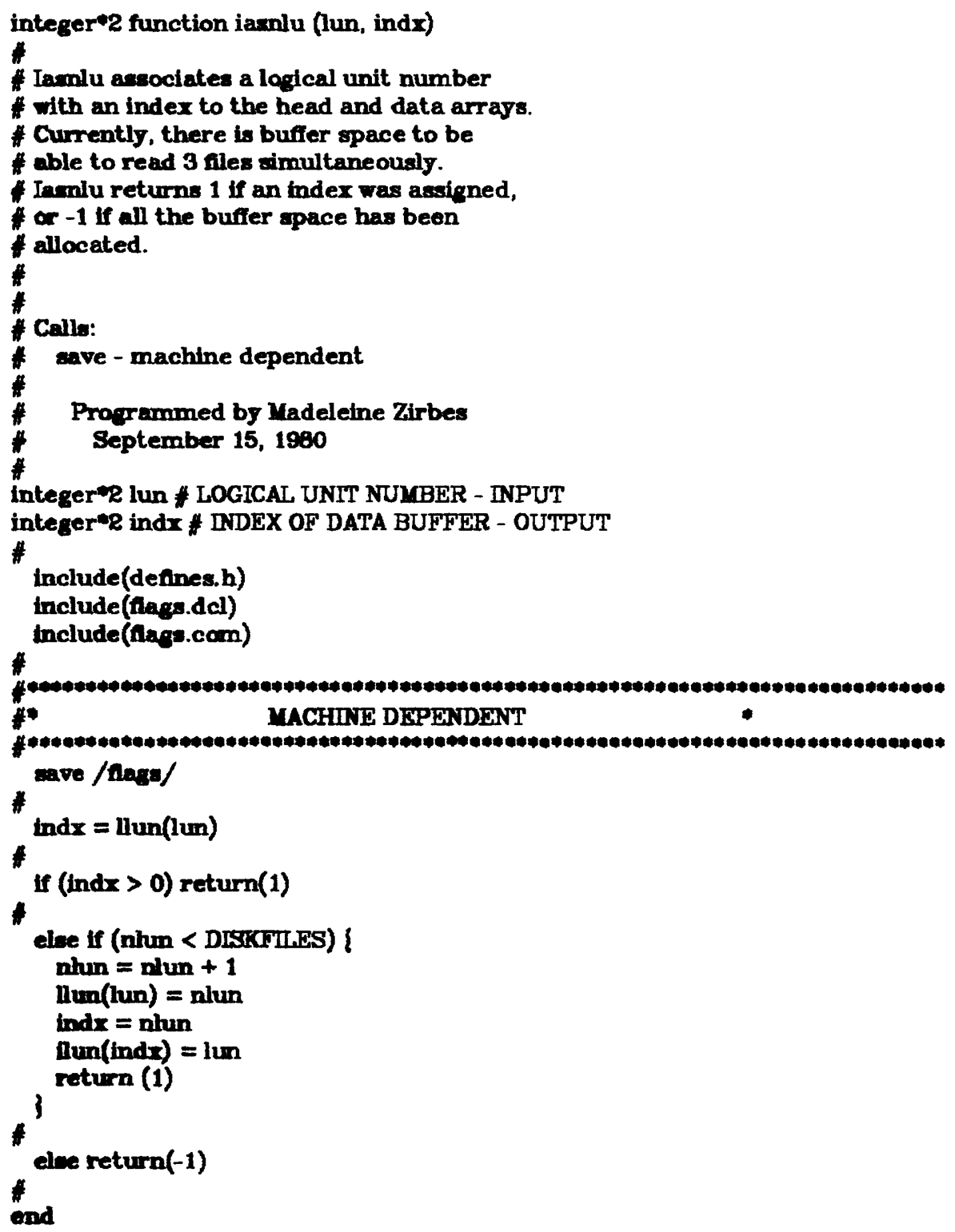


INITFL.RATFOR

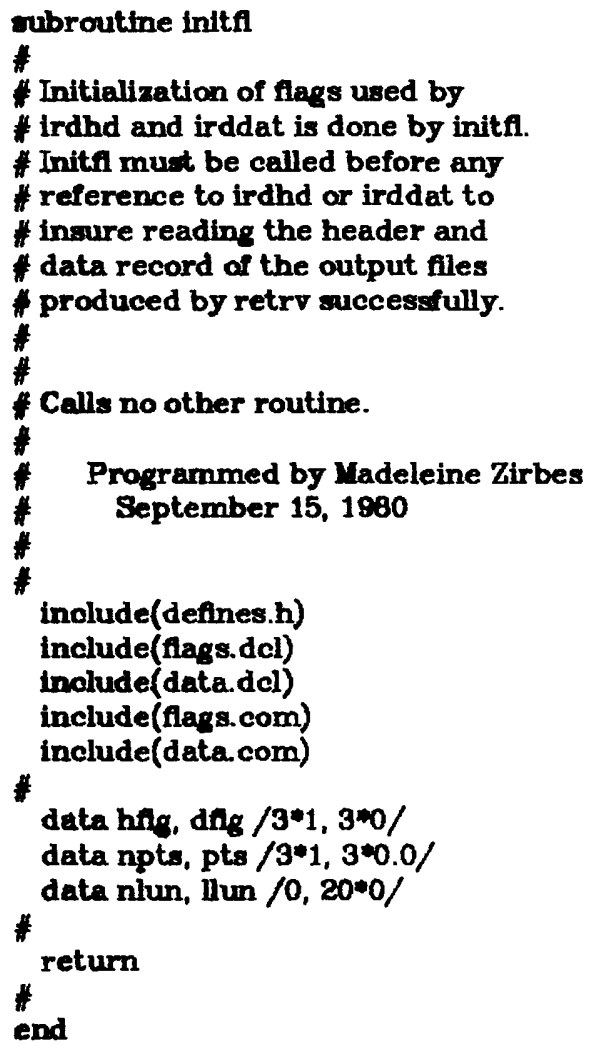


RDHEAD.RATFOR

subroutine rdhead(lun, indx, tf)

4

\# Rdhead reads a header record from

* logical unit number lun. tf $=1$

\# If this is the last record of

\# the file.

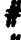

* Calls no other routine.

Programmed by Madeleine Zirbes

September 15, 1880

integer*2 lun \# LOGICAL UNIT NUMBER - INPUT

integer 22 indx \# ARRAY INDEX INTO THE DATA WUL BE READ - INPUT

integer'2 tf \# END OF DATA FIAG - OUTPUT

\#

integer"2

include (defines.h)

Include(head.dcl)

include(head.com)

\#

$t f=0$

read(lun) (code(i,ind $x), i=1,4),(\operatorname{chn}(i, \operatorname{lnd} x), 1=1,4)$.

lat (indx), lon(ind $x$ ), elev(ind $x$ ).

arear(indx), sdofy(indx), shour(tndx), smin(indx),

meco(ind $x$ ), rate(ind $x$ ), ao(ind $x$ ),

np(indx). (poles( 1 ,ind $x), i=1, n p($ ind $x)$ ).

$n$ (indx). (zeroes(i,ind $x$ ), $i=1, n z$ (ind $x$ )

t

* If te equals ONE,

* THIS IS A NULJ HEADER RECORD -

* IAST RECORD IN THE FILE

if $(a 0$ (ind $x)==0.0)$ tf $=1$

$\#$

return

\#

end 
RDDATA.RATFOR

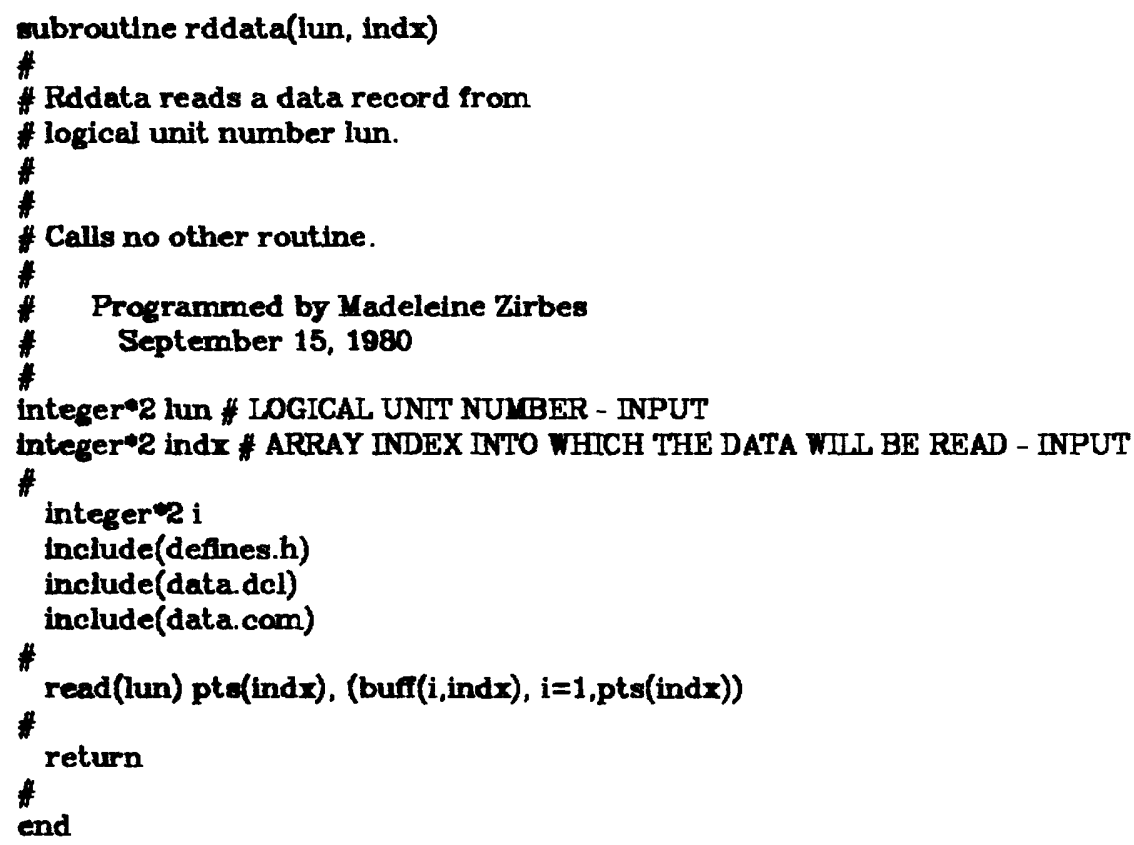




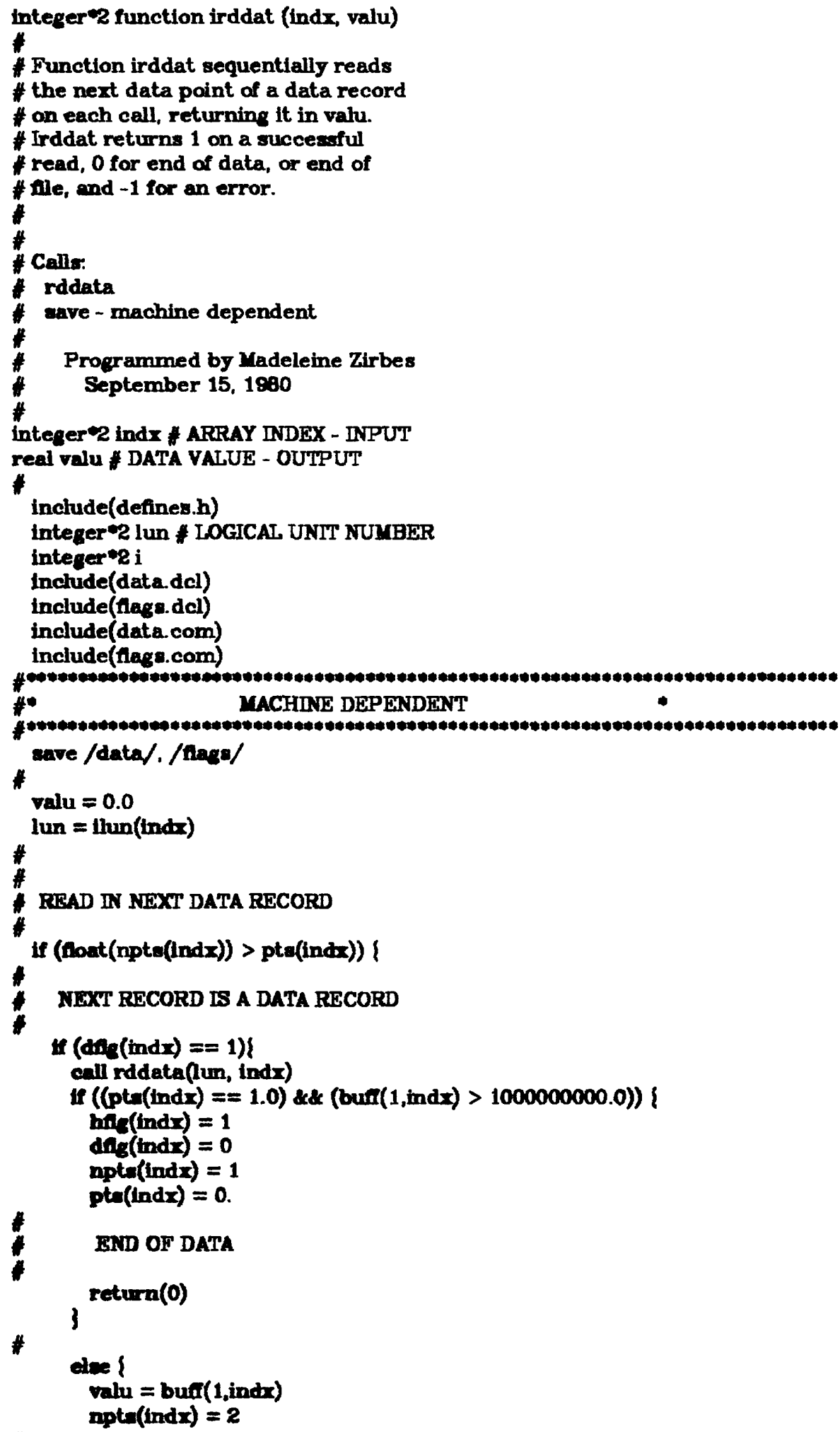




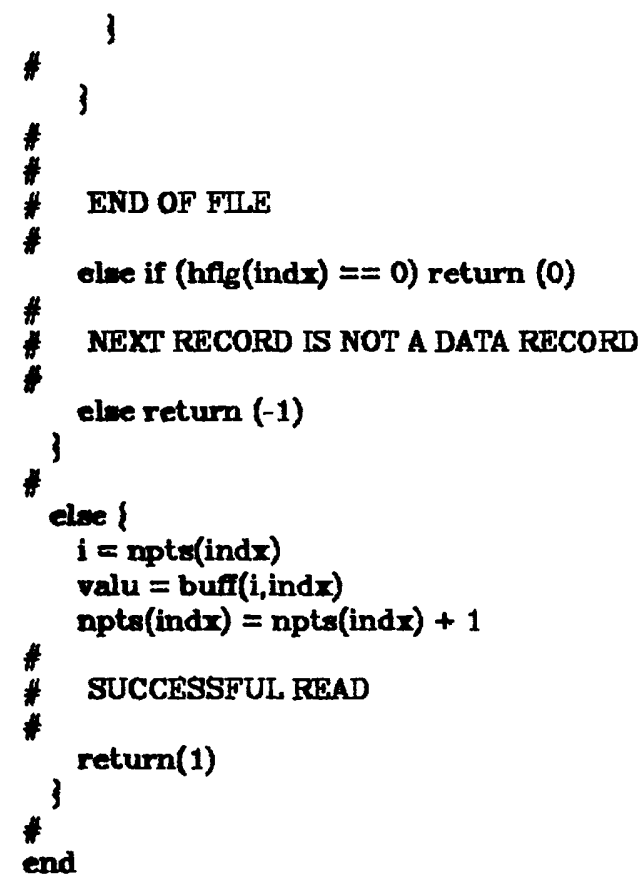


IRDHD.RATFOR

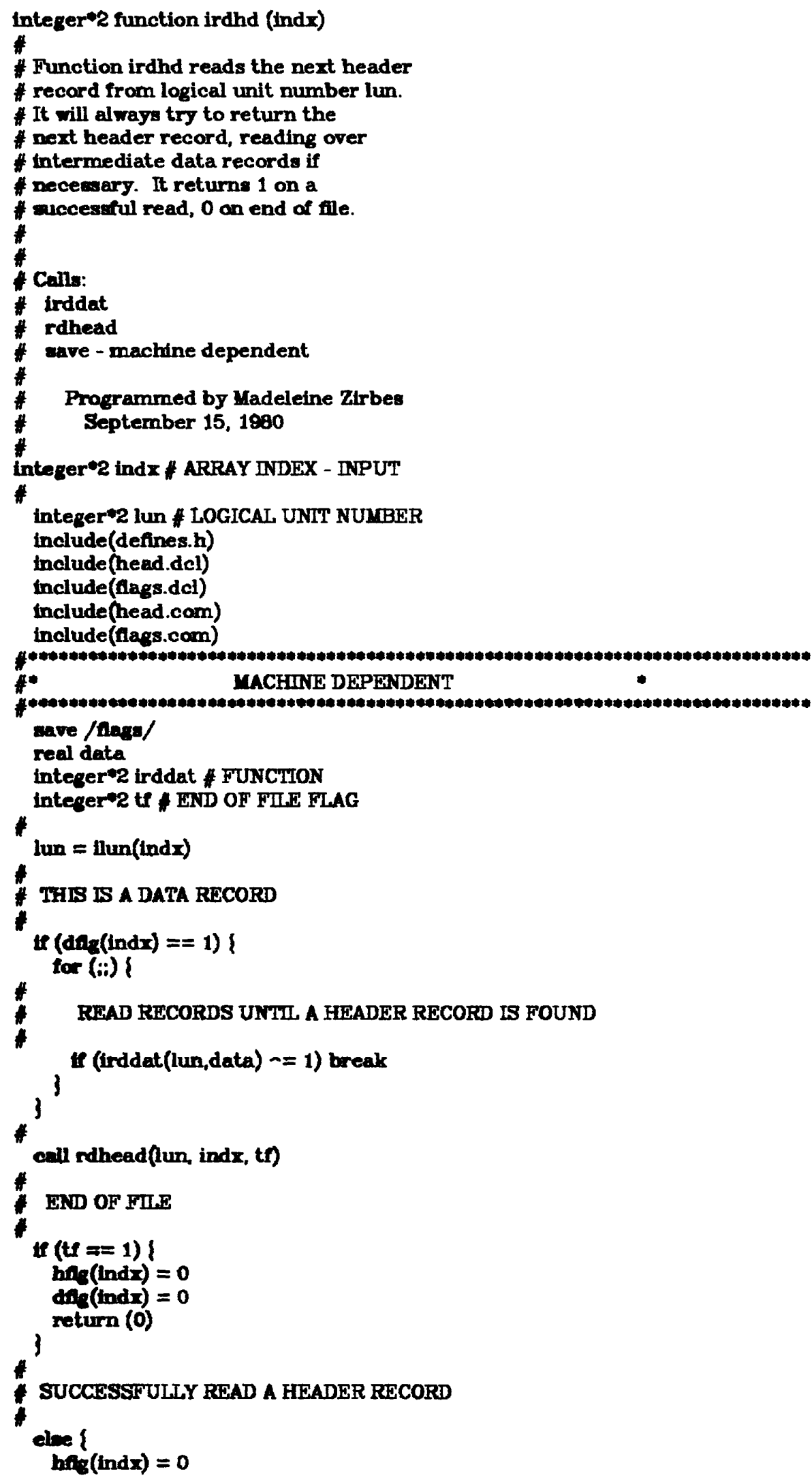


- 99 -

$\operatorname{dffg}($ ind $x)=1$

return (1)

$8=?$ 
RESP.RATFOR

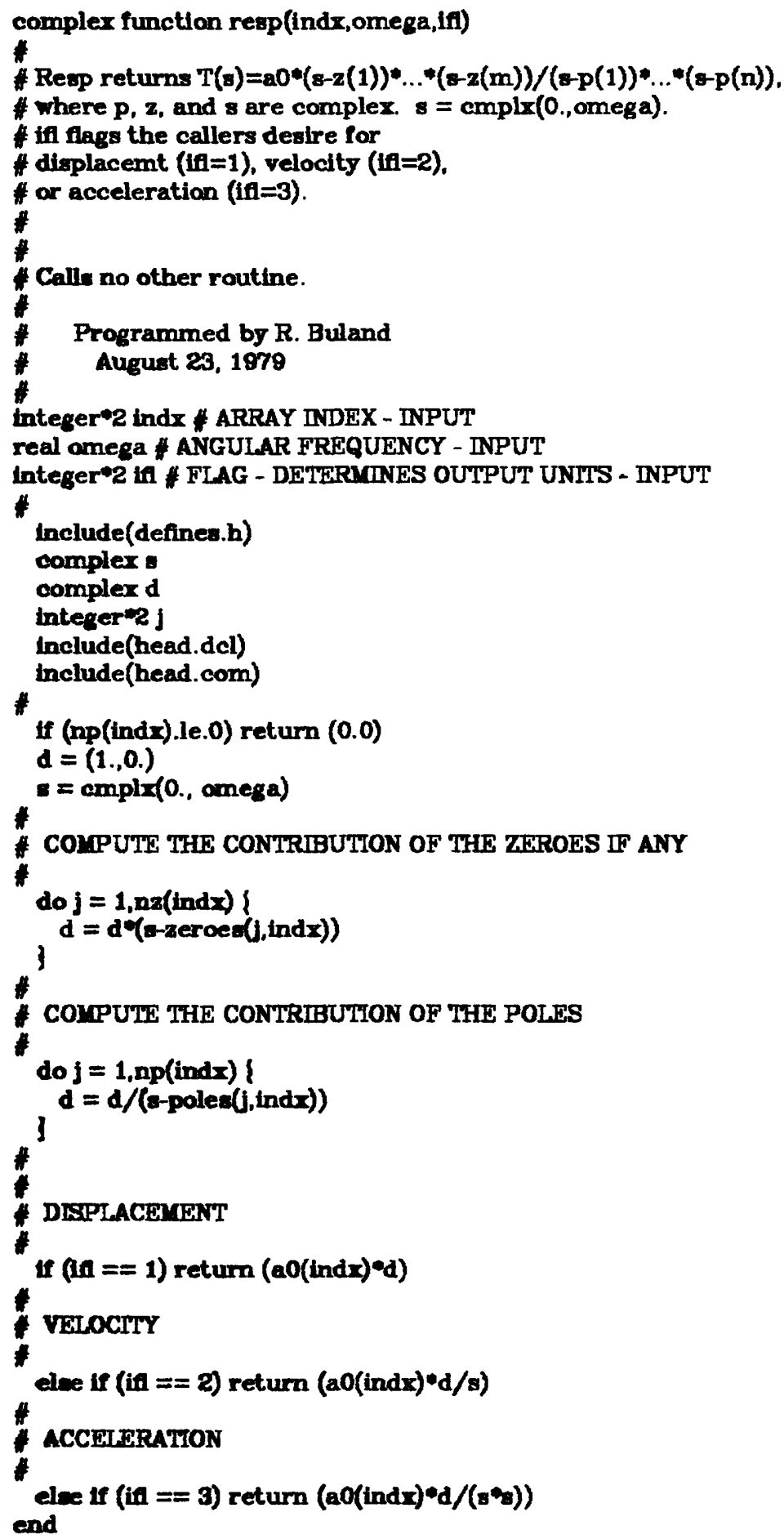


$-101-$

APPENDIX B

FORTRAN CODE

The Fortran code was generated from the Ratfor code in Appen$\operatorname{dix} \mathrm{A}$. 
NDTSUM MAIN

C

C COMMAND NDTSUM PRODUCES A SUMMARY

C OF THE DATA WRITEN ON A NETWORK

C DAY TAPE. THIS INCLUDES A LITERAI.

C DUMP OF ALL DATA LOGS, AND A LIST

C OF START AND END TIMES OF THE

C CONIINUOUS DATA RECORDED FOR EACH

C STATTON AND EACH INSTRUMENT.

C

C NDISUM READS FROM THE STANDARD

C INPUT (I.OGICAL UNIT NUMBER 5)

C FOR THE TAPE DRIVE UNIT NUMBER.

C NO PROMPT IS GIVEN TO THE USER.

C

C CAILIS:

C MATT - USER SUPPIIED

C MRETRN - USER SUPPIIED

C SUM

C

C PROGRAMMED BY MADELEINE ZIRBES

C SEPTEMBER 15, 1980

c

C

C TAPE HEADER INFORMATION

C

C STATION ID

INTEGER ID

C INSTRUMENT

INTEGER INSTR

C YEAR

INIEGER YEAR

C DAY OF YEAR

INTEGER DOFY

C HOUR

INTEGER HOUR

C MINUTE

INIEGER MTN

C SECOND

INTEGER SEC

C MIIISECONDS

REAL MSS

C FORMAT II

INTEGER NFORM

C NUMBER OF CHANNELS

INTEGER NCHAN

C NUMBER OF SAMPLES

INTEGER NSAMP

C SAMPLING RATE

REAL RATE

C

C START TTME OF CONTINOUS DATA

C

C YEAR

INTEGER SYR

C DAY OF YEAR

INTEGER SDY

C HOUR

INTEGER SHR

C MINUTE

INTEGER SMN

C SECONDS

INTEGER SSEC 


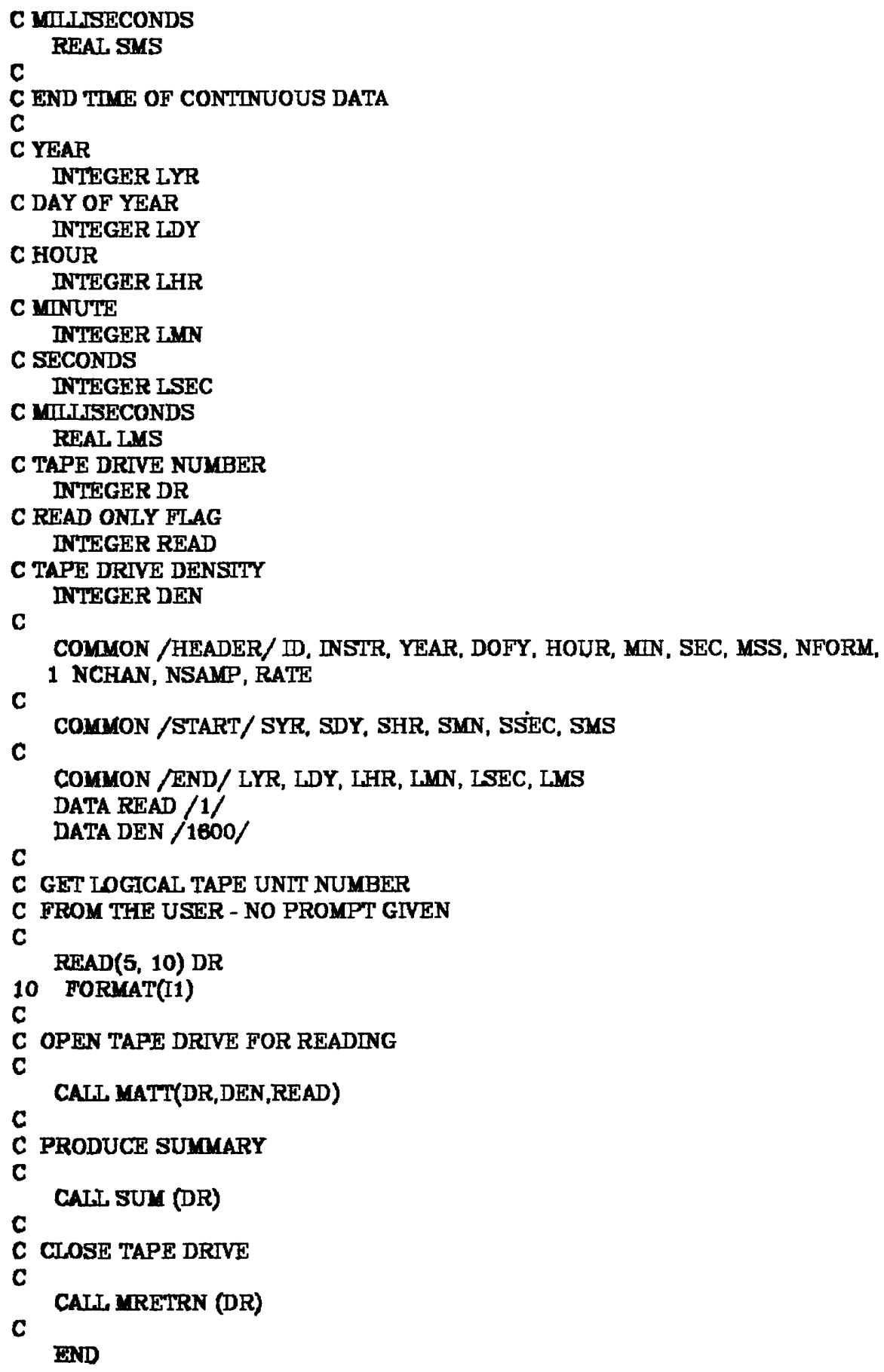


NDSTUM SPECIFIC ROUTINES

SUM.FORTRAN

$$
\text { c }
$$

C SUM PRODUCES A SUMMARY OF THE

C DATA CONTAINED ON A NETWORK

C DAY TAPE.

C

C

C CAIJS:

C GETLIN

C HEAD

c MAGRD - USER SUPPLIED

C NDTPRT

C PRBUF

C STNDRD

C TSET

c

C PROGRAMMED BY MADELEINE ZIRBES

C SEPTEMBER 15, 1980

C

C TAPE DRIVE LOGICAL UNIT

C INTEGER DR

C

C TAPE HEADER INFORMATTON

C

C STATTON ID

INTEGER ID

C INSTRUMENT

INTEGER INSTR

C YEAR

INTEGER YEAR

C DAY OF YEAR

INTEGER DOFY

C HOUR

INTEGER HOUR

C MINUTE

INTEGGER MIN

C SECOND

INTEGER SEC

C MUI.JSECONDS

REAL MSS

C FORMAT ID

INTEGER NFORM

C NUMBER OF CHANNETS

INTEGER NCHAN

C NUMBER OF SAMPIES

INTEGER NSAMP

C SAMPLING RATE

REAL RATE

C

C START TIME OF CONTINOUS DATA

C

C YEAR

INTEGER SYR

C DAY OF YEAR

INTEGER SDY

C HOUR

INTEGER SHR

C MINUTE 


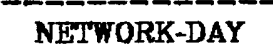

TAPE LOG (ASCII)

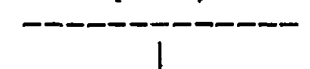

c

C

C

C

C

C

C

C

C

C

c

c

C

C

C

C

C

\section{IP DATA LOG}

(ASCII)

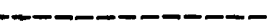

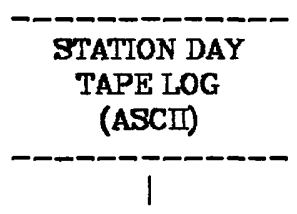

EOF

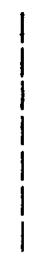

IP DATA
SP DATA LOG

(ASCII)

EOF

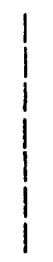

SP DATA

EOF<smiles>II</smiles>

EOF

STATTON DAY

TAPE LOG

(ASCII)

C FOR

23000 CONTINUE

C

C

READ NETWORK DAY TAPE LOG

$I=$ MAGRD(DR, NWORDS, DATA, NR)

IF(.NOT.(I.EQ. O))GOTO 23003

C GOTO 23002

C PRINT OUT NETWORK DAY TAPE LOG

C EISE

23003 CONTTNUE

IF(.NOT.(I .EQ. 1))GOTO 23005 CAIL PRBUF(IDATA)

C EISE

GOTO 23008

23005 CONTINUE

WRITE $(6,10)$

10 FORMAT(1X, 'ERROR READING - NETWORK DAY TAPE LOG.')

$$
\text { RETURN }
$$

23008 CONTINUE

23004 CONTINUE

COTO 23000

C ENDFOR

23002 CONTINUE

C

C READ STATTON IOG

C

IF(.NOT.(MAGRD(DR, NWORDS, IDATA, NR) .NE. 1))GOTO 23007 WRITE $(6,12)$

12 FORMAT(1X, 'ERROR READING - STATTON LOG EXPECIED.') REHTURN 


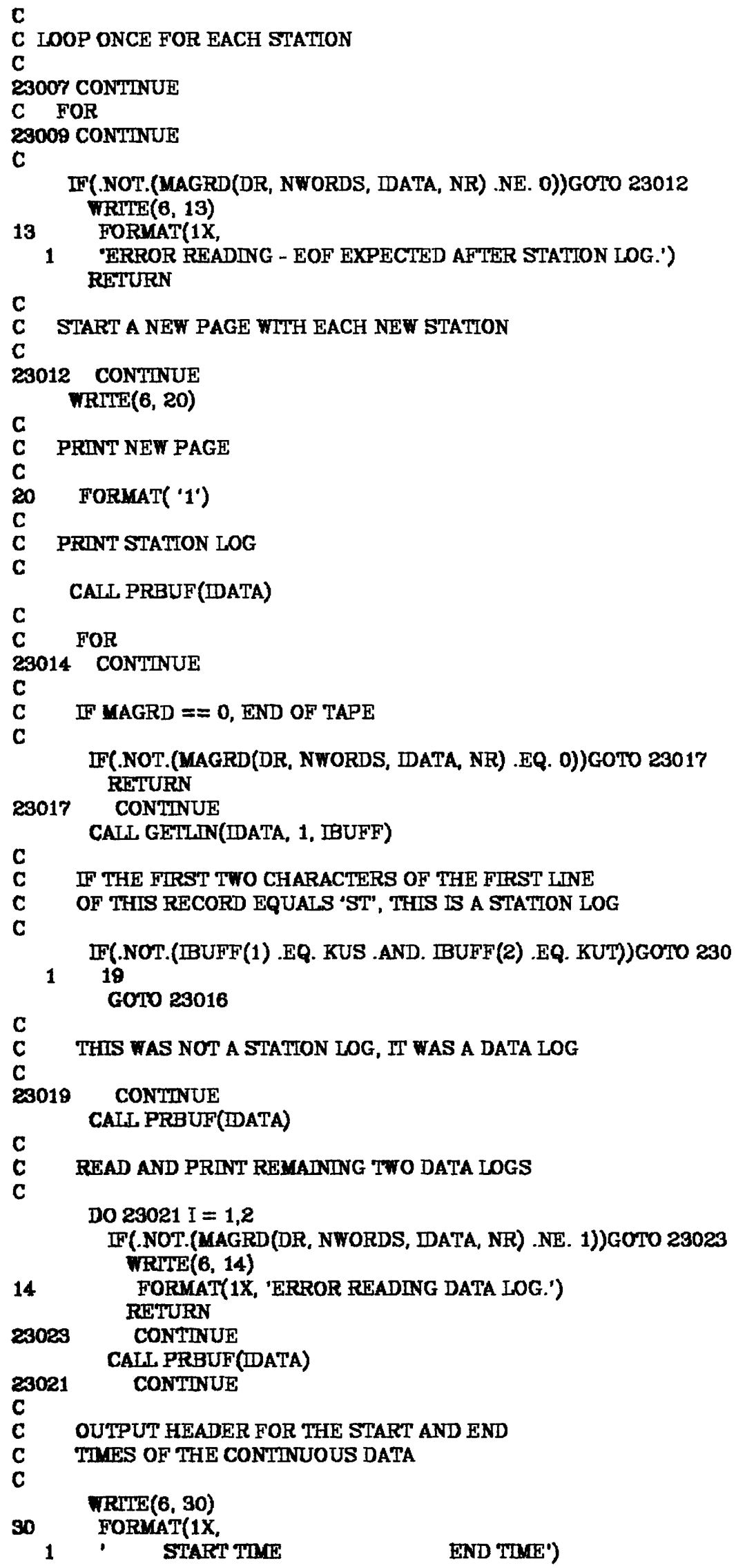




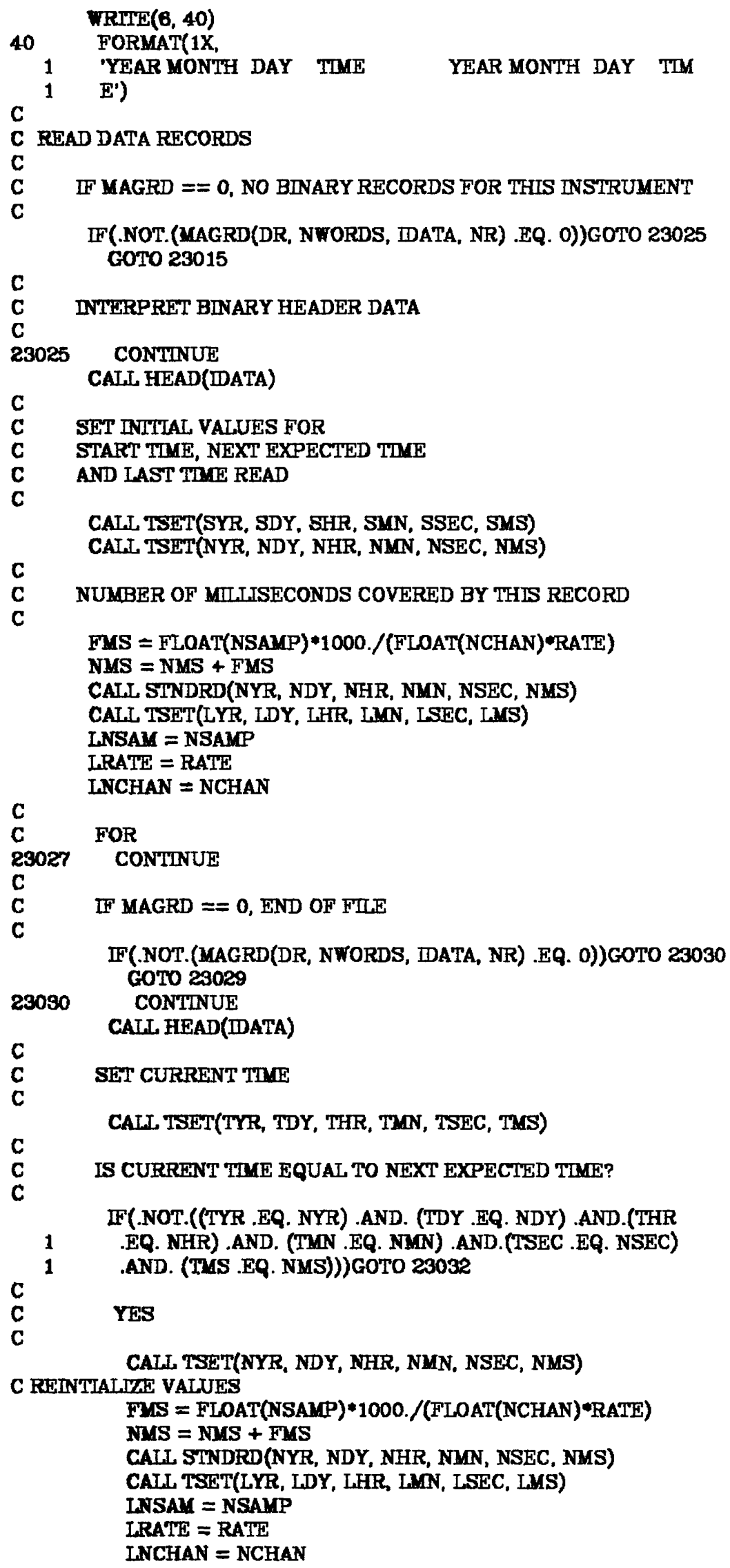




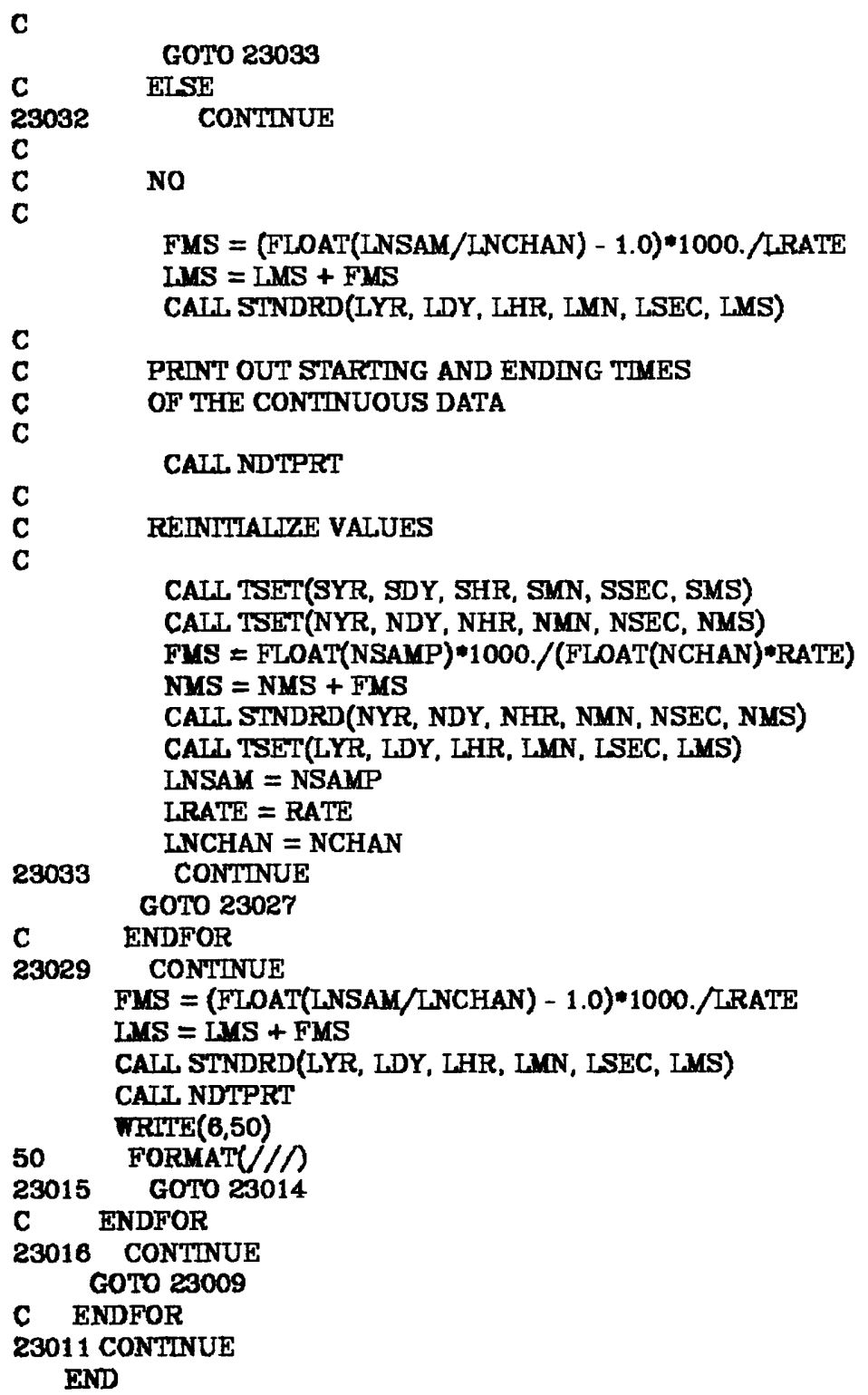


PRBUF.FORTRAN

C

SUBROUTINE PRBUF(IDATA)

C PRBUF PRINTS ONE ASCII RECORD(2000 CHARACTERS) -

C 25 IJNES, 60 CHARACTERS PER LINE.

C

C CAIIS:

C GETLIN

C

C PROGRAMNED BY MADELEINE ZIRBES

C SEPTEMBER 15, 1980

C

C DATA RECORD - INPUT

INTEGER IDATA(1)

C SET TO HOLD 16000 BITS OF TAPE INPUT RECORD

c

INTEGER I

INTEGER J

C

INTEGER IBUFF(80)

DO $23000 I=1,25$

CALI GETLIN(IDATA, I, IBUFF)

WRTIE $(6,10)$ (IBUFF(J), $J=1,80$ )

10 FORMAT(1X, 80A1)

29000 CONTINUE

C

C

RETURN

END 
NDTPRT.FORTRAN

C

SUBROUTINE NDTPRT

C NDTPRT WRITES OUT THE START AND

C END TTMES IN COMMON BLOCKS

C /START/ AND /END/.

c

C NDTPRT USES A COLON (:) IN

C THE FORMAT STATEMENT. THIS MAY

C NOT BE AVAIIABLE ON ALL MACHINES.

C

C

C CALIS:

C DAYMO

C

C PROGRAMMED BY MADELEINE ZIRBES

C SEPTEKMBER 15, 1980

c

C

C

C START TIME OF CONTINOUS DATA

C

C YEAR

INTEGER SYR

C DAY OF YEAR INTEGER SDY

C HOUR INTEGER SHR

C MINUTE INTEGER SMN

C SECONDS INTEGER SSEC

C MIIISECONDS

C REAL SIS

C END TIME OF CONTINUOUS DATA

C

C YEAR

INTEGER LYR

C DAY OF YEAR INTEGER IDY

C HOUR INTEGER IHIR

C MTNUTE INTEGER IMN

C SECONDS INTEGER ISEC

C MILIISECONDS

REAL IMS INTEGER SMONTH, SDAY, LMONTH, IDAY

C FUNCTION INTEGER DAYMO

C ERROR CODE

C

C COMMON /START/ SYR, SDY, SHR, SMN, SSEC, SMS

C COMMON /END/ LYR, LDY, LHR, LMN, LSEC, IMS

IFR = DAYMO(SDY, SMONTH, SDAY, SYR)

c ITR = DAYMO(LDY, LMONTH, LDAY, LYR)

WRITE(6, 10) SYR, SMONTH, SDAY, SHR, SMN, SSEC, SMS, LYR, LMONTH, 
$-112$

1 LDAY, LHR, LMN, ISEC, LMS

C

C * MACHINE DEPENDENT

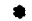

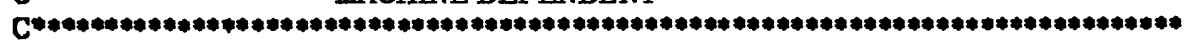

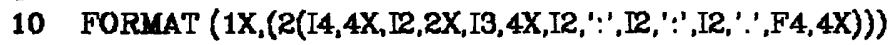

C

C

RETURN

END 
RETRV MAIN

C

C COMMAND RETRV FETCHES DATA FROM A

C NETWORK DAY TAPE MOUNTED ON

C LOGICAL TAPE UNIT N AND GENERATES

C OUTPUT FIIES.

C RETRV READS FROM THE STANDARD

C INPUT (LOGICAL UNIT NUMBER 5)

C THE TAPE DRIVE UNTT NUMBER,

C AND THEN CONTINUES TO READ THE

C USER'S REQUESTS.

C NO PROMPT IS GIVEN TO THE USER

C FOR THIS INFORMATION.

C

C

C CALIS:

C CLSOUT

C GETDAT

C GETRCT

C INITBD

C MATT - USER SUPPIIED

C MRETRN - USER SUPPIEIED

C OPNOUT

c

C PROGRAMMED BY MADELEINE ZIRBES

C SEPTEMBER 15, 1980

C

C

C REQUEST INFORMATTON

C 50 - MAXTMUM NUMBER OF REQUESTS ALIOWED

C

C REQUEST INFORMATION

C NUMCBER OF REQUESTS

INTEGER NREQ

C STATION CODE - CHARACTER STRTNG INTEGER RCODE $(50,4)$

C INSTRUMENT ID INTEGER RINSTR(50)

C YEAR

INTEGER RYEAR(50)

C MONTH

C DAY

INTEGER RMONTH(50)

INTEGER RDAY(50)

C HOUR

INTEGER RHOUR(50)

C MINUTE

INTEGER RMIN(50)

C SECONDS

INTEGER RSEC (50)

C LETHGTH IN SECONDS

REAL RLEN(50)

C IOGICAL UNIT NUMBER

INTEGER RLUN $(50,3,3)$

C INSTRUMENT CODE - CHARACTER STRING

INTEGER RCHN $(50,3,3,4)$

C STATTON FIAG

C

INTEGER RFLG(50)

C TAPE HEADER INFORMATION

C

C STATTON ID

INTEGER ID 
C INSTRUMENT INTEGER INSTR

C YEAR INTEGER YEAR

C DAY OF YEAR INTEGER DOFY

C HOUR INIEGER HOUR

C MINUTE INIEGER MIN

C SECOND INTEGER SEC

C NMIIJSECONDS REAL MSS

C FORMAT ID INTEGER NFORM

C NUMBER OF CHANNELS INTEGER NCHAN

C NUMBER OF SAMPLES INTEGER NSAMP

C SAMPLING RATE REAL RATE

C C STATION INFORMATION

C

C STATION CODE - CHARACTER STRING INTEGER CODE(4)

C NUMBER OF POLES, 20 LARGEST NUMBER OF SRO POLES INTEGER NP

C NUMBER OF ZEROES, 10 IARGEST NUMBER OF SRO ZEROES INTEGER NZ

C NUMBER OF CHANNEIS INTEGER ICHAN

C CONSTANT REAL AO(3)

C CALIBRATION CONSTANT REAL CAL(3)

C STATION EIEVATION REAL ELEV

C STATHON LATTTUDE REAL IAT

C STATION LONGITUDE REAL ION

C POLES COMPLEX POLES(20,3)

C ZEROES COMPLEX ZEROES(10,3)

c

C STAET TTME OF DATA

C

C YEAR INTEGER SYEAR

$C$ DAY OF YEAR INTEGER SDOFY

C HOUR INIEGER SHOUR

C MINUTHS INTEGER SMDN

C SECONDS

c REAL SSECS

C LOGICAL UNIT NUMBER FOR EACH OUTPUT FIIE INTEGER IUN (3,3)

C INSTRUMENT CODE FOR EACH OUTPUT FIIE INTEGER CHN(3,3,4) 


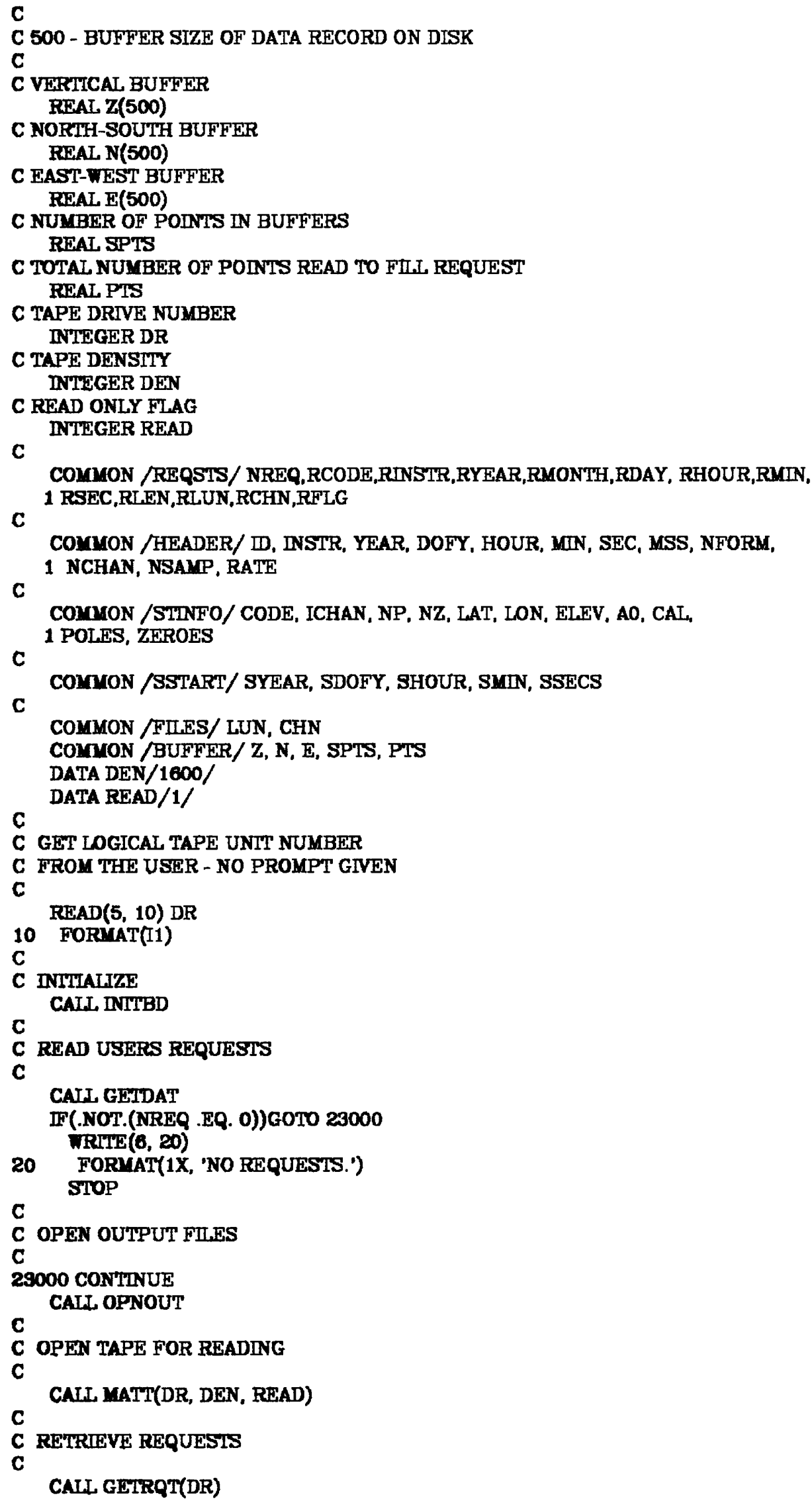


-116 -

C

C CLOSE OUTPUT FILES

C

C CALL CISOUT

C CLOSE TAPE DRIVE

C C CALL MRETRN(DR) END 
RETRV SPECIFIC ROUTINES

INITBD.FORTRAN

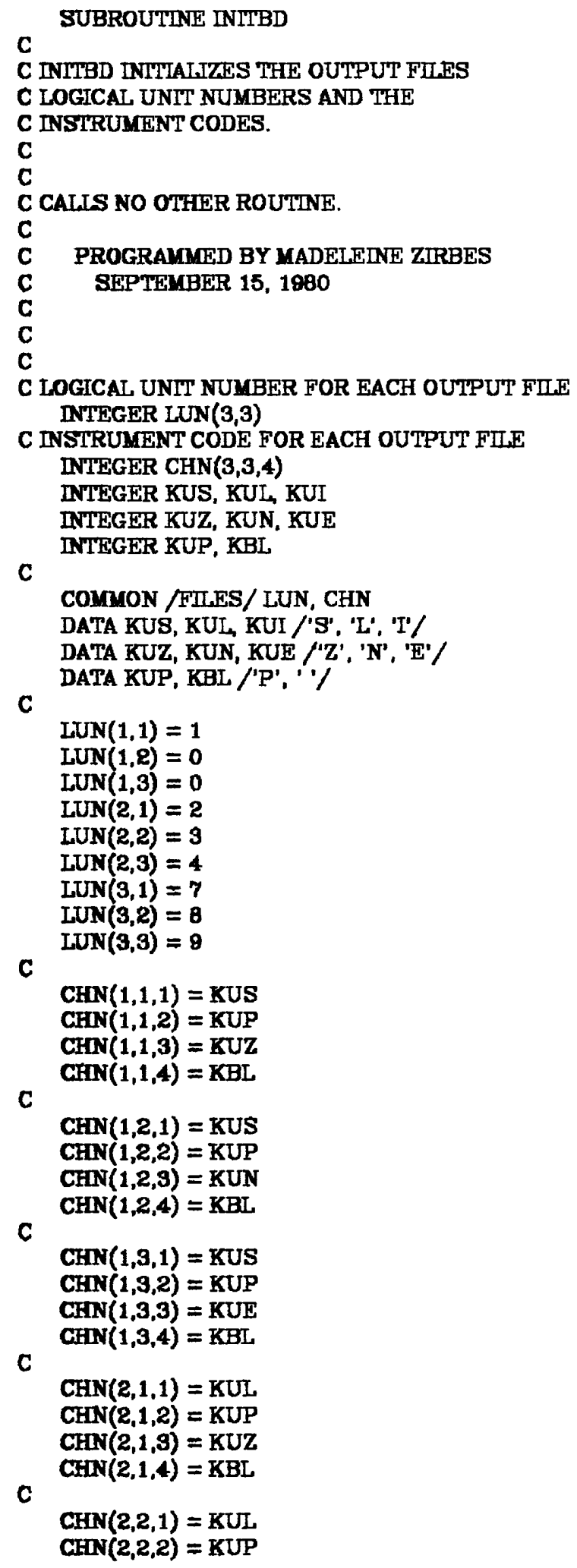

$\operatorname{CHN}(1,2,1)=\mathrm{KUS}$ $\operatorname{CHN}(1,2,2)=$ KUP $\operatorname{CHN}(1,2,3)=\mathrm{KUN}$ 


\footnotetext{
$\operatorname{CHN}(2,2,9)=\mathrm{KUN}$

c $\operatorname{CHN}(2,2,4)=\mathrm{KBL}$

$\operatorname{CHN}(2,3,1)=\mathrm{KUL}$ $\operatorname{CHN}(2,3,2)=$ KUP $\operatorname{CHN}(2,3,3)=K U E$

$\operatorname{CHN}(3,3,1)=K U I$ $\operatorname{CHN}(3,3,2)=\mathrm{KUP}$ $\operatorname{CHN}(3,3,3)=K U E$

c $\operatorname{CHN}(3,3,4)=\mathrm{KBI}$

C RETURN

END
} 
C

SUBROUTINE GETDAT

C GETDAT SCANS THE STANDARD INPUT,

C (LOGICAL UNIT 5) READING REQUESTS.

C SCANNING STOPS UPON READING A STATION

C CODE OF STOP.

C

C

C CAIIS:

C FFT

C GETSTR

C ICPYCH

C

C PROGRAMMED BY MADEI.EINE ZIRBES

C SEPTEMBER 15, 1980

C

C

C

C REQUEST INFORMATION

C 50 - MAXIMUM NUMBER OF REQUESTS ALLOFED

C

C REQUEST INFORMATION

C NUMBER OF REQUESTS INTEGER NREQ

C STATTON CODE - CHARACTER STRING INTEGER RCODE $(50,4)$

C INSTRUMMENT ID INTEGER RINSTR(50)

C YEAR

INIEGER RYEAR(50)

C MONTH

C DAY INTEGER RMONTH(50) INTEGER RDAY(50)

C HOUR INTEGER RHOUR(50)

C MINUTE INTEGER RMIN(50)

C SECONDS INTEGER RSEC(50)

C IENGTH IN SECONDS RAEAL RIEN(50)

C LOGICAL UNIT NUMBER INTEGER RIUN $(50,3,3)$

C INSTRUMIENT CODE - CHARACTER STRING INTEGER RCHN(50,3,3,4)

C STATTON FLAG

c INTEGER RFLG(50)

C LOGICAL UNIT NUMBER FOR EACH OUTPUT FILE INTEGER LUN $(3,3)$

C INSTRUMENT CODE FOR EACH OUTPUT FIIE INTEGER CHN $(3,3,4)$ INTEGER KUA, KUE, KUI, KUL, KUN INTEGER KUO, KUP, KUS, KUT, KUZ INTEGER KCMA, KBL INTEGER I, J, K, I, M, MM INTEGER IFL. INTEGER F'ID INTEGER INSTR(4) INTEGER CODE(4) INTEGER IINE(80) 


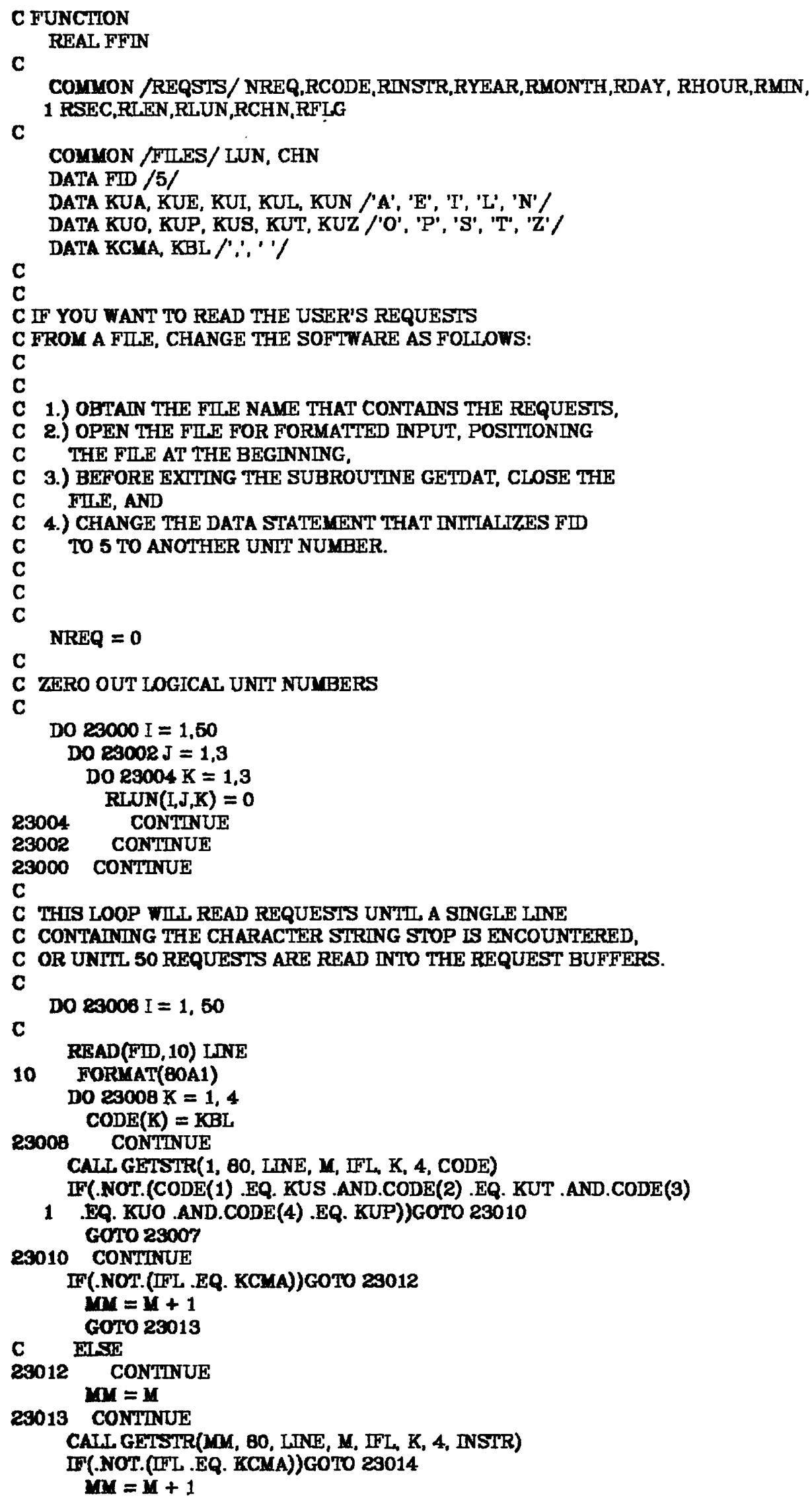




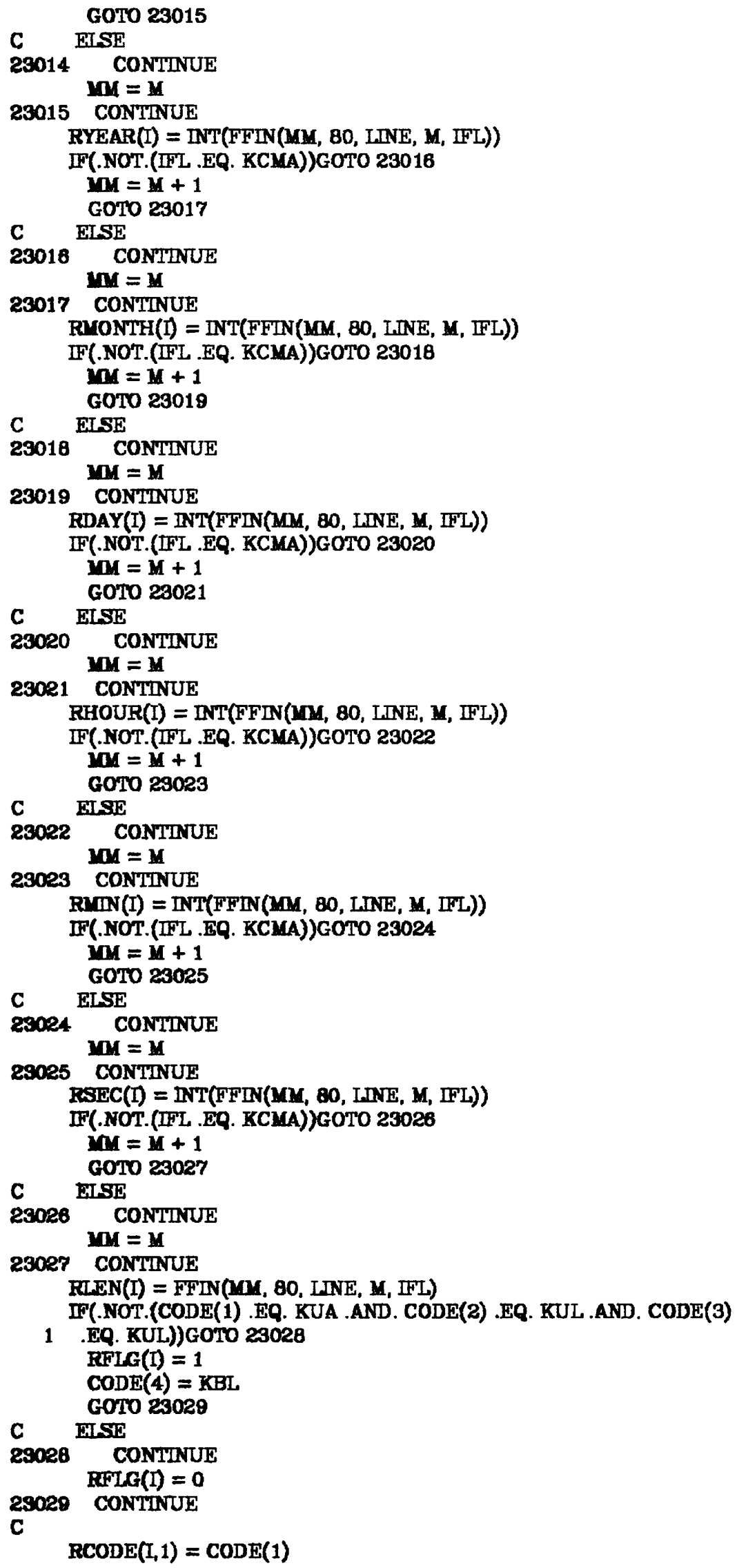




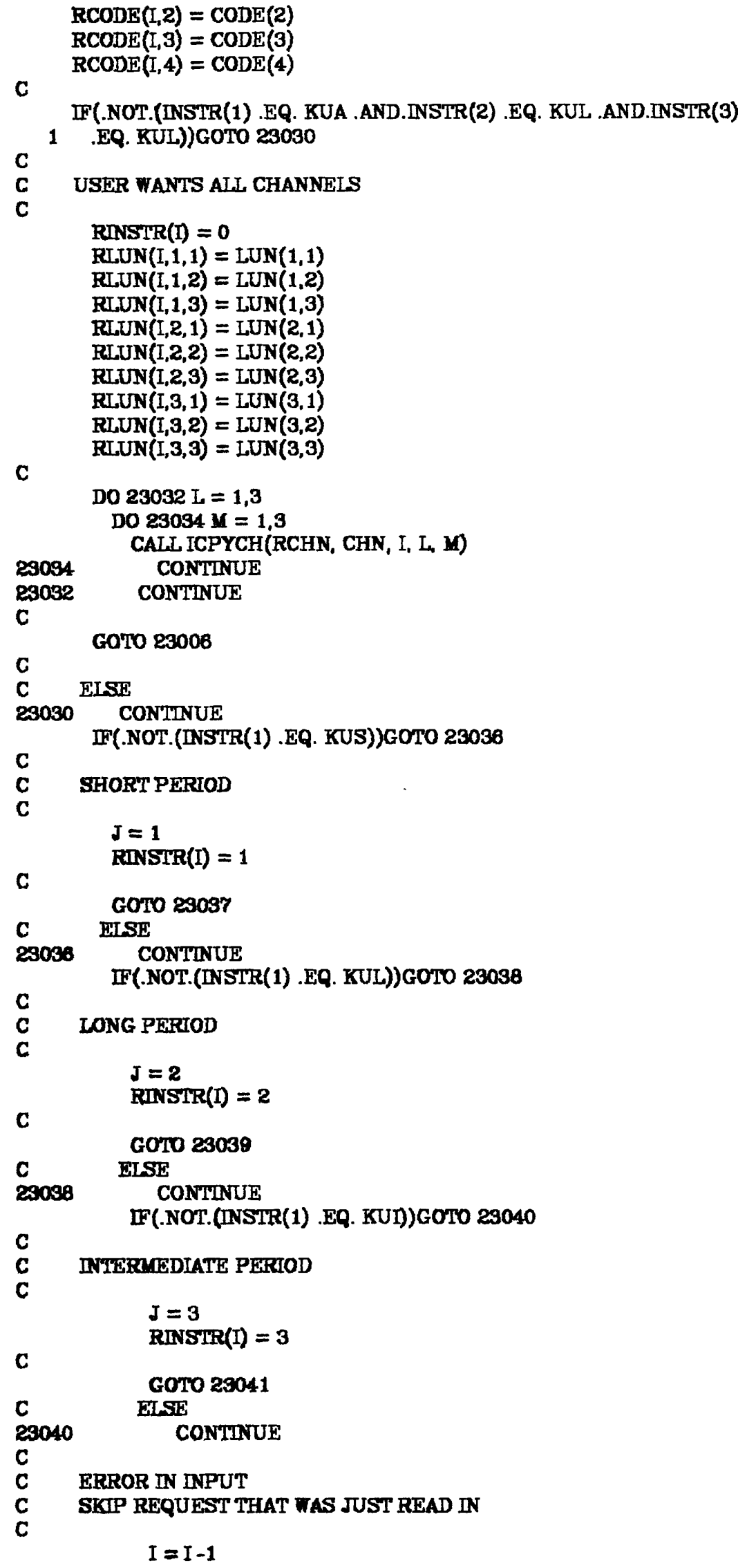




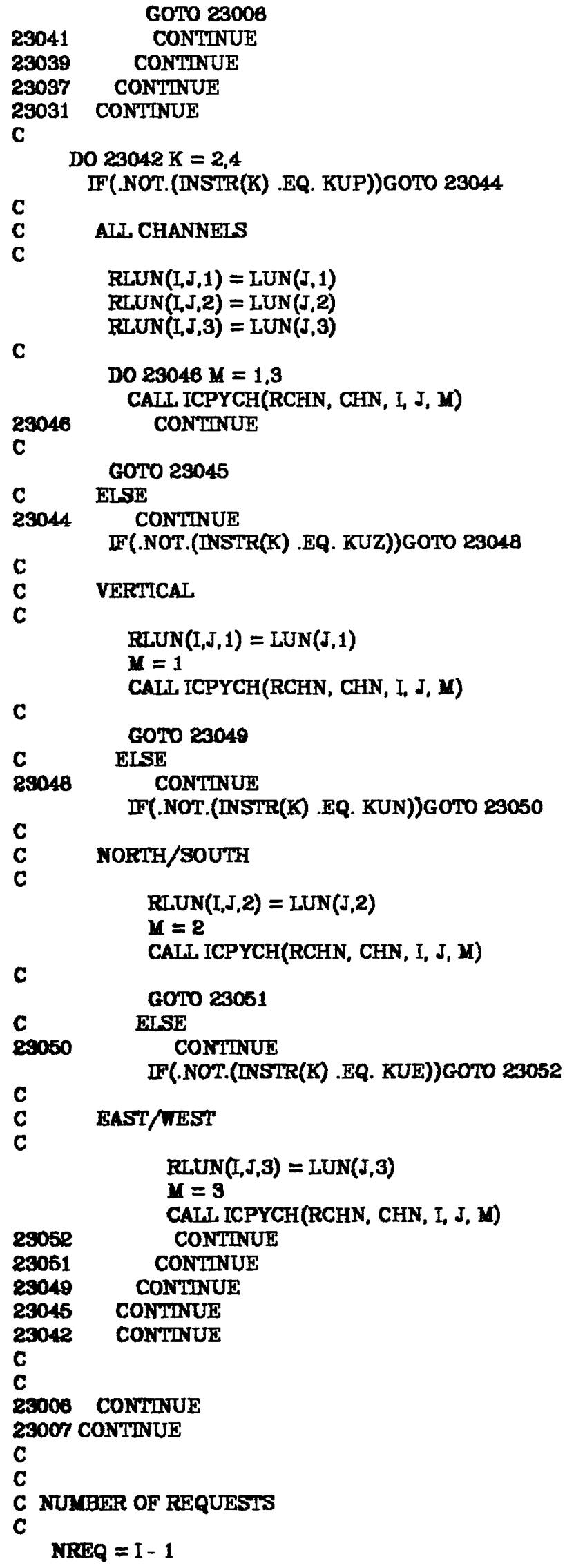


$-124-$

c

RETURN

END 
OPNOUT.FORTRAN

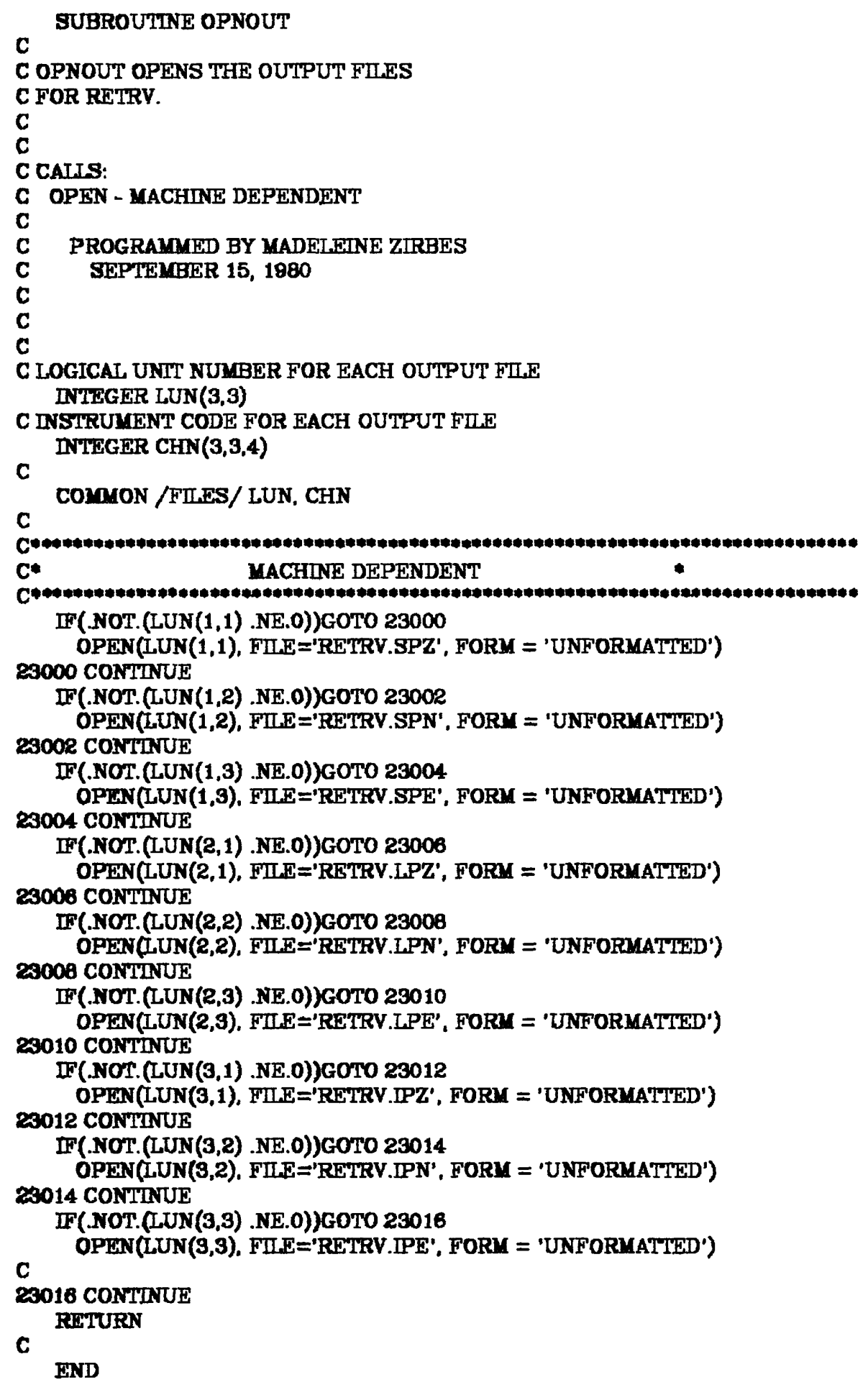




\section{CISOUT.FORTRAN}

C

SUBROUTINE CISOUT

C CLSOUT WRITES OUT A NULL HEADER

C RECORD TO EACH OPEN OUTPUT FIIE

C (OPENED BY OPNOUT) BEFORE

C CLOSING THAT FILE.

C

C

C CAIIS:

C CLOSE - MACHINE DEPENDENT

C

C PROGRAMMED BY MADELEINE ZIRBES

C SEPTEMBER 15, 1980

C

C

C

C LOGICAL UNIT NUMBER FOR EACH OUTPUT FIIE INTEGER LUN (3,3)

C INSTRUMENT CODE FOR EACH OUTPUT FIIE INTEGER CHN $(3,3,4)$

C LOOP INDEX

INTEGER I, J, K

C INTEGER ZERO INTEGER IZERO

C INTEGER ONE INTEGER IONE

C CHARACTER STRING INTEGER CODE(4)

C LOGICAL UNIT NUMBER INTEGER L

C FLOAT ZERO

REAL FZERO

C

COMMON /FIIES/ LUN, CHN

DATA ZEERO, IONE $/ 0,1 /$

DATA FZERO $/ 0.0 \%$

C

DATA CODE /'E', 'N', 'D', '

DO $23000 I=1,3$

DO $23002 \mathrm{~J}=1,3$

IF(.NOT.(IUN(I,J) .NE. O))GOTO 23004

C WRTE OUT NULC HEADER $\mathrm{L}=\mathrm{LUNN}(\mathrm{I}, \mathrm{J})$

WRIIE(L) (CODE(K), K=1,4), (CODE(K), K=1,4), FZERO,

1 FZZERO, FZERO, IZERO, IZERO, IZERO, IZERO, FZERO, FZERO,

1 FZEXRO, IONE, CMPLX(FZERO, FZERO), IONE, CMPIXX(FZERO,

1 FZERO)

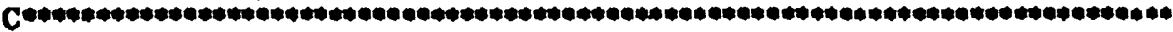

C.

MACHINE DEPENDENT

-

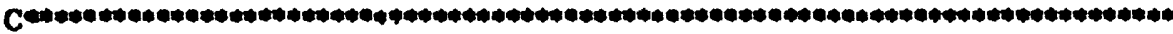

CLOSE(L)

$\operatorname{LUN}(I, J)=0$

29004 CONTINUE

23002 CONITNUE

28000 CONTTNUE

C

C

RRETURN

END 
C

SUBROUTINE GETRQT(DR)

C GETRQT READS A NETWORK DAY TAPE

C AND TRYS TO SATISFY EACH REQUEST.

C

C GETRQT USES A COLON IN A FORMAT

C STATEMENT. THIS MAY NOT BE

C AVATIABLES ON ALL MACHINES.

C

C

C CALLS:

C DAYMO

C FILREQ

C GETCAL

C GETCPZ

C GETIIN

C HEAD

C ISTRERQ

C MAGRD - USER SUPPIIED

C RDNTLG

C REQ

C SE'IFLG

C STADAT

C

C PROGRAMMED BY MADELEINE ZIRBES

C SEPTEMBER 15, 1980

C

C TAPE DRIVE LOGICAL UNIT - INPUT

INTEGER DR

c

C

C TAPE HEADER INFORMATTON

C

C STATTON ID

INTEGER ID

C INSTRUMENT

INTEGER INSTR

C YEAR

INTEGER YEAR

C DAY OF YEAR

INTEGER DOFY

C HOUR

INTEGER HOUR

C MINUTE

INTEGER MIN

C SECOND

INTEGER SEC

C MTIISECONDS

REAL MSS

C FORMAT ID

INTEGER NFORM

C NUMBER OF CHANNEIS

INTEGER NCHAN

C NUMBER OF SAMPLES

INTEGER NSAMP

C SAMPLING RATE

c

RERAL RATE

C REQUEST INFORMATTON

C 50 - MAXMUUM NUMBER OF REQUESTS ALLOWED

C

C REQUEST INFORMATION 
C NUMBER OF REQUESTS INTEGER NREQ

C STATTON CODE - CHARACTER STRING INTEGER RCODE $(50,4)$

C INSTRUMENT ID INTEGER RINSTR(50)

C YEAR INTEGER RYEAR(50)

C MONTH INTEGER RMONTH(50)

C DAY INTEGER RDAY(50)

C HOUR INTEGER RHOUR(50)

C MINUTE INTEGER RMIN(50)

C SECONDS INTEGER RSEC(50)

C IENGTH IN SECONDS REAL RIEN(50)

C LOGICAL UNIT NUMBER INTEGER RLUN $(50,3,3)$

C INSTRUMENT CODE - CHARACTER STRING INTEGER RCHN $(50,3,3,4)$

C STATTON FLAG

c INTEGER RFIG(50)

C STATTON INFORMATION

C

C STATION CODE - CHARACTER STRING INTEGER CODE(4)

C NUMBER OF POLES, 2O LARGEST NUMBER OF SRO POLES INTEGER NP

C NUMBER OF ZEROES, 10 LARGEST NUMBER OF SRO ZEROES INTEGER NZ

C NUMBER OF CHANNEIS INTEGER ICHAN

C CONSTANT REAL AO(3)

C CALIBRATION CONSTANT RIEAL CAL(3)

C STATION ELEVATION REAL ELEV

C STATION LATITUDE REAL IAT

C STATION IONGITUDE REAL LON

$C$ POLES CONPLEX POLES $(20,3)$

C ZEROES

C COMPIEXX ZEROES $(10,3)$

C START TMM OF DATA

C

C YEAR

DNTEGER SYEAR

C DAY OF YEAR INTEGER SDOFY

C HOUR INTEGER SHOUR

C MTINUTES INTEGER SMTN

C SECONDS REAJ, SSECS

C 

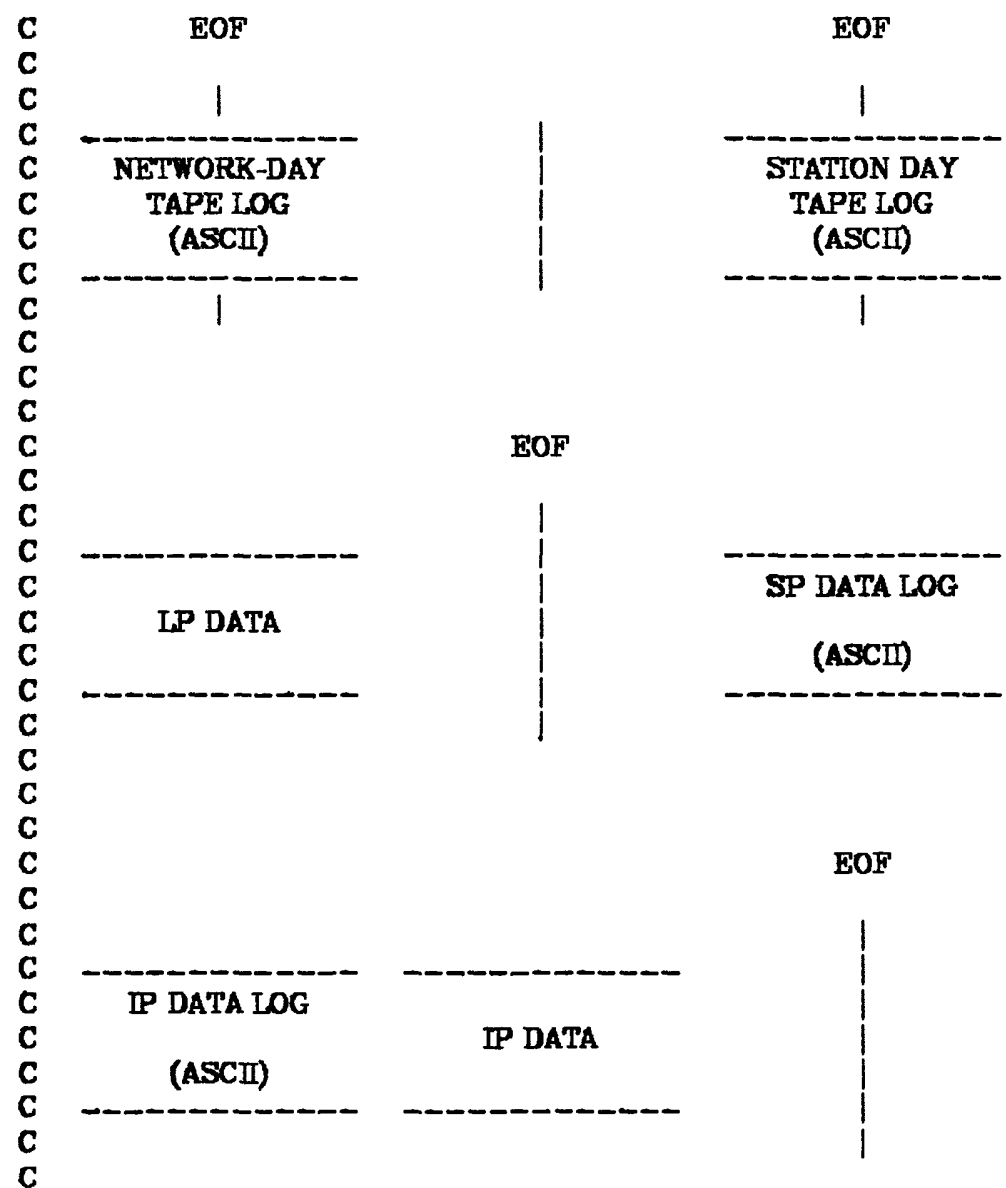

EOF

EOF
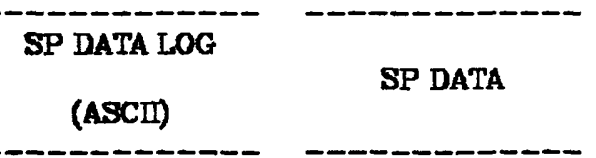

IP DATA LOG

(ASCII)

(ASCII)
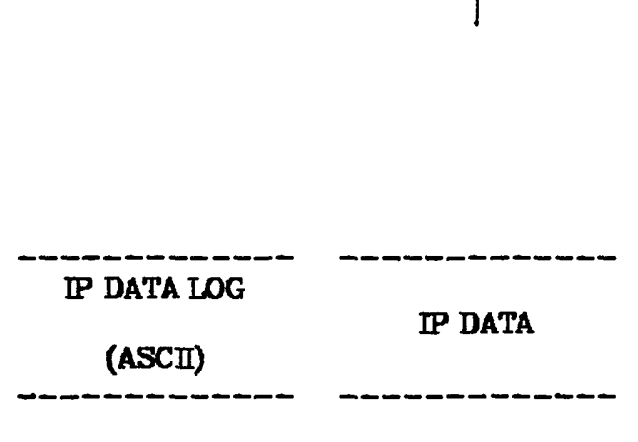

EOF

EOF

C FOR

23000 CONTINUE

C

C READ NETWORK DAY TAPE LOG(S)

C

$I=\operatorname{MAGRD}(D R$, NTORDS, IDATA, NR)

IF(.NOT.(I .EQ. O))GOTO 23008

GOTO 23002

C

C GET DATA FROM NETWORK LOG

C

C ETSE

28008 CONTMTUE

IF(.NOT.(I .EQ. 1))GOTO 23005

CALT RDNTLG(IDATA)

C GOISE

GOTO 29006

23005 CONTHUE

WRTTE(6,10)

10 FORMAT(1X, 'ERROR READING - NETTORK DAY TAPE LOG.')

29006 CONTTNUE

23004 CONTINUE

GOTO 23000

C ENDFOR

23002 CONTINUE

C

C READ STATION LOG

C

IF(.NOT.(MAGRD(DR, NWORDS, IDATA, NR) .NE. 1))GOTO 23007 WRITE(6,11) 
C 500 - BUFFER SIZE OF DATA RECORD ON DISK

C

C VERTTCAL BUFFER REAL Z(500)

C NORTH-SOUTH BUFFER REAL N(500)

C EAST-WEST BUFFER REAL E(500)

C NUMBER OF POINTS IN BUFFERS REEAL SPTS

C TOTAL NUMBER OF POINTS READ TO FILL REQUEST REAL PTS

C FUNCTION INTEGER MAGRD

C ERROR CODE INTEGER IERR INTEGER NWORDS INTEGER NR

C BINARY DATA INTEGER IDATA(1000)

C SET TO HOLD 16000 BISS OF TAPE RECORD INPUT

C USER REQUEST BETNG SATISFIED INTEGER INDX INTEGER I, J INTEGER IBUFF(80) INTEGER KUS, KUT INTEGER RDFLAG

C FUNCIION INIEGER REQ

C FUNCTON INTEGER GETCAL

C FUNCTION INTEGER DAYMO

C FUNCTION INTEGER ISTREQ INIEGER FLAG

C MONTH INTEGER KALL(4)

C DAY TNTEGER MON INTEGER DY

C INSTRUMENT INTEGER ITNSTR REAL RETART

C COMMON /HEADER/ ID, INSTR, YEAR, DOFY, HOUR, MIN, SEC, MSS, NFORM,

c 1 NCHAN, NSAMP, RATE

COMMON /REQSTS/ NREQ,RCODE,RINSTR,RYEAR,RMONTH,RDAY, RHOUR,RMIN, C 1 RSEC,RLEN,RLUN,RCHN,RFIG

COMMON /STINFO/ CODE, ICHAN, NP, NZ, LAT, ION, ELEV, AO, CAL,

c 1 POLES, ZEROES

COIMON /SSTART/ SYEAR, SDOFY, SHOUR, SMTN, SSECS

COIMON /BUFFER/ Z, N, E, SPTS, PIS

DATA NWORDS/1000/

DATA KALE /'A', 'L', 'L', '"

DATA KUS, KUT / $\mathrm{S}$ ', 'T"/

DATA CODE $/$, , , ?

C NETWORK DAY TAPE FORMAT

C 
11 FORMAT(1X, 'ERROR READING - STATION LOG EXPECTED.')

C

C IOOP ONCE FOR EACH STATTON

C

23007 CONTINUE

C FOR

23009 CONTINUE

C

IF(.NOT.(MAGRD(DR, NWORDS, IDATA, NR) .NE. 0))GOTO 23012 WRTYE $(6,12)$

12 FORMAT (1X,

1 'ERROR READING - EOF EXPECTED AFTER STATTON LOG.')

C

C BEFORE READING NEW STATTON LOG,

C CHECK TO SEE IF THERE ARE ANY

C MORE REQUESTS TO SATISFY.

C IF NOT, RETURN TO NATN PROGRAM.

C

C

C SET REQUEST FLAG TO TRUE FOR ALL REQUESTS THAT

C HAVE THE REQUESTED STATION EQUAL TO 'ALL'

C

Q9O12 CONTINUE

CAIL SETFLG(KALL, 1)

C

C SET REQUEST FLAG TO FALSE FOR ALL REQUESTS THAT

C MATCH THE PREVIOUS STATION CODE. EVEN IF THEY

C WERE NOT SATTSFED, NO MORE DATA FOR THAT STATTON

C REMAINS ON THE TAPE.

C

C

CALL SETFLG(CODE, 0)

C ARE THERE ANY OUTSTANDING REQUESTS?

C

FIAG $=0$

DO $23014 \mathrm{I}=1$,NREO

IF(.NOT.(RFLG(I) .EQ. 1))GOTO 23016

FLAG $=1$

GOTO 23015

2S016 CONTINUE

23014 CONTINUE

29015 CONTINUE

IF(.NOT.(FLAG .EQ. O))GOTO 23018

BETURN

C GET STATION DATA - LATTIUDE, LONGHUDE, ELEVATION, INSTRUMENT TYPE

C

23018 CONTINUE

C

CALL STADAT(DATA, CODE, IAT, LON, ELEV)

C FOR

29020 CONTINUE

C

c IF MAGRD $==0$, END OF TAPE

C IF MAGRD $==0$, END OF TAPE

IF(.NOT.(MAGRD(DR, NWORDS, DDATA, NR) .EQ. O))GOTO 230Z3 RETURN

C

C IF THE FIRST TWO CHARACTERS OF THE FIRST LINE OF THE BUFFER

c EQUAIS 'ST, THIS IS A STATTON IOG

C

23023 CONTINUE

CALL GETL IN(DATA, 1, IBUFF)

1

IF(.NOT.(IBUFF(1) .EQ. KUS .AND. IBUFF(2) .EQ. KUT))GOTO 230 


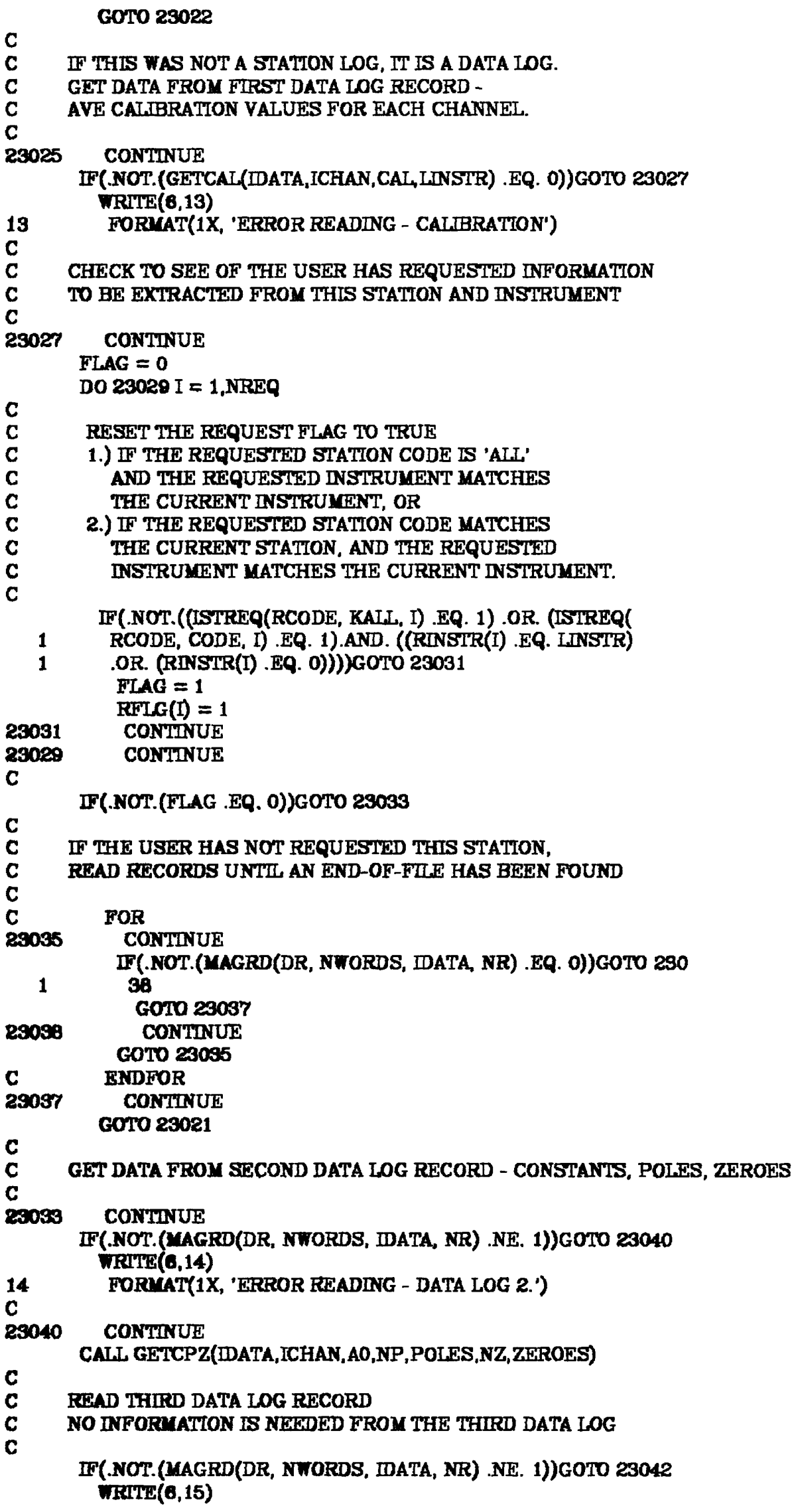


$-134-$

\author{
23059 CONTINUE \\ 23045 GOTO 23044 \\ C ENDFOR \\ 23046 CONTINUE \\ C \\ 23021 GOTO 23020 \\ C ENDFOR \\ Z3OR2 CONTINUE \\ C \\ GOTO 23009 \\ C ENDFOR \\ Z3011 CONTTNUE \\ C \\ END
}




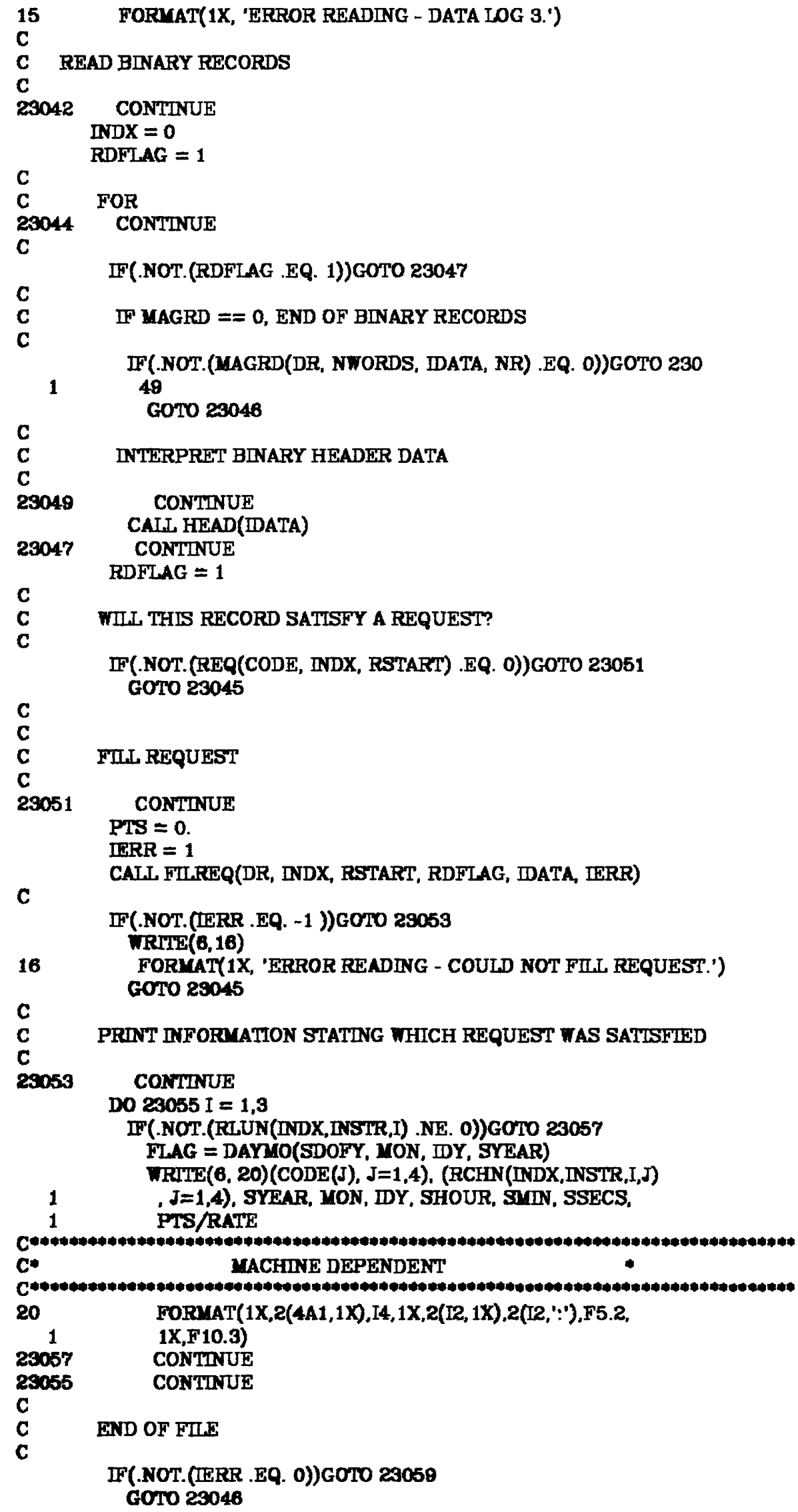


RDNTLG.FORTRAN

C

SUBROUTINE RDNTLG (IDATA)

C RDNTLG SCANS A NETWORK DAY TAPE

C LOG FOR THE STATIONS RECORDED

C ON THE TAPE. RDNTLG SETS A FIAG FOR

C THE REQUEST INFORMATION.

C

C

C CAIJS:

C GETLTN

C GETSTR

C SETFLG

C

C PROGRAMMED BY MADELEINE ZIRBES

C SEPTEMBER 15, 1880

C

C DATA RECORD

c INTEGER IDATA(1)

TNTEGER KBL

C STATION CODE - CHARACTER STRING INTEGER CODE(4)

C CHARACTER BUFFER

INTEGER IBUFF(80)

INTEGER M, I, IFL

C IOOP INDEX

INTEGER ITNE

C

DATA KBL $/ \%$

DO 23000 IINE $=6,25$

CALL GETLIN(IDATA, LINE, IBUFF)

C

C CHECK COLUMN ONE FOR STATION CODE

C

DO $23002 I=1,4$

$\operatorname{CODE}(I)=\mathrm{KBL}$

29008 CONITUE

CAIL GETSTR(6, 9, IBUFF, M, IFL, I, 4, CODE)

IF(.NOT.(CODE(1) .EQ. KBL .AND.CODE(2) .EQ. KBL .AND.CODE(3)

1 .EQ. KBL .AND.CODE(4) .EQ. KBL))GOTO 23004

GOTO 23001

23004 CONTWNUE

C

CAIL SETFLG(CODE, 1)

C CHECK COLUMN TWO FOR STATION CODE

C

$10023006 \mathrm{I}=1,4$

$\operatorname{CODE}(\mathrm{I})=\mathrm{KBL}$

28008 CONTTNUE

CALL GETSTR(41, 44, IBUFF, M, IFL, I, 4, CODE)

IF(.NOT. ( .NOT.(CODE(1) .EQ. KBL .AND.CODE(2) .EQ. KBL .AND.

1 CODE(3) .EQ. KBL .AND.CODE(4) .EQ. KBL)))GOTO 23008

CATL SETFLG(CODE, 1)

23008 CONTINUE

23000 CONTINUE

23001 CONTINUE

C

c

RETURN

END 
c

SUBROUTINE SETFLG (CODE, FIAG)

C SETFLG IOOPS THROUGH THE REQUESTS

C AND SETS RFLG(I) TO FLAG IF CODE AND

C RCODE ARE EQUAL.

C

C

C CAIJS:

C ISTREQ

C

c PROGRAMMED BY MADELEINE ZIRBES

C SEPTEMBER 15, 1880

C

C STATION CODE - INPUT

INTEGER CODE(4)

C STATION FLAG - INPUT

c

INTEGER FLAG

C

C REQUEST INFORUATION

C 50 - MAXTMUM NUMBER OF REQUESTS ALLOWED

C

C REQUEST INFORMATION

C NUMBER OF REQUESTS INTEGER NREQ

C STATION CODE - CHARACTER STRTNG

INTEGER RCODE $(50,4)$

C INSTRUUTENT ID

INTEGER RINSTR(50)

C YEAR

INTEGER RYEAR(50)

C MONTH

C DAY

TNTEGER RMONTH(50)

INTEGER RDAY(50)

C HOUR

INTEGER RHOUR(50)

C MUNTE

NTIEGER RITN(50)

C SECONDS

INTEGER RSEC(50)

C IENGTH IN SECONDS

REAL RLEN(50)

C IOGICAL UNIT NUMBER

INTEGER RLUN (50,3,3)

C INSTRUMENT CODE - CHARACIER STRING INTEGER RCHN $(50,3,3,4)$

C STATION FLAG

INTEGER RELG(50)

INTEGER I

DITEGER ISTREQ

C

COMMON /REQSTS/ NREQ,RCODE,RINSTR,RYEAR,RMONTH,RDAY, RHOUR,RMTN,

1 RSEC,RLEN,RLUN,RCHN,RFLG

C

DO $23000 \mathrm{I}=1, \mathrm{NREQ}$

IF(.NOT.(ISTREQ(RCODE, CODE, I) .EQ. 1))GOTO 23002 RFIG (I) = FIAG

23002 CONTINUE

83000 CONTINUTE

C

REITRN 
C

END 
C

SUBROUTINE STADAT(IDATA, CODE, IAT, LON, ELEV)

C STADAT SCANS A STATTON LOG FOR THE

C STATION CODE, IATTTUDE, LONGTTUDE, AND

C ELIEVATION.

C

C

C CAIIS:

C FFIN

C GETIIN

C GETSTR

C

C PROGRAMMED BY MADELEINE ZIRBES

C SEPTTMMBER 15, 1980

C

C DATA RECORD - INPUT

INTEGER IDATA(1)

C STATTON CODE - OUPUT INTEGER CODE(4)

C STATION IATTTUDE - OUTPITT REAL IAT

C STATION LONGITUDE - OUTPUT REAL ION

C STATION ELEVATION - OUTPUT REAL ELEV

C

INTEGER KUS, KUW, KBL

INTEGER I

INTEGER Y

DNTEGER IFL

INTEGER IBUFF(B0)

INTEGER L

C FUNCTION

RREAL FFTN

C

DATA KUS, KUW, KBL /'S', 'W', ' '/

DO 22000 I $=1,4$

CODE $(\mathrm{I})=\mathrm{KBL}$

23000 CONTINUE

C

CALI, GETLIN(IDATA, 5, IBUFF)

c

CAIJ GETSIR(20, 80, IBUFF, Y, IFL, I, 4, CODE)

C IATTTUDE

C

IAT $=$ FFTN $(36,60$, IBUFF, M, IFL)

IF(.NOT.(IFL .EQ. KUS)) GOTO 23002

ILAT $=-$ LAT

C

C LONGTUDE

C

28002 CONTINUE

$\mathrm{L}=\mathbf{M}+\mathbf{I}$

ION $=$ FFTN(L, 80, IBUFF, M, IFL)

IF(.NOT.(IFL .EQ. KUW)) GOTO 29004 LON $=-$ LON

C

C RLEVATION

C

2SOO4 CONTANUE

$L=M+1$

EILV $=\operatorname{FFTN}\left(L_{1}, 80\right.$, IBUFT, M, IFL $)$ 
- 139 -

C

RETURN

END 
GETCAL FORTRAN

C

INTEGER FUNCTION GETCAL(DATA, ICHAN, CAL, LINSTR)

C FUNCTION GETCAL SCANS DATA LOG 1 FOR

C CALTBRATION CONSTANT(S).

C THE CAIJPRATTON CONSTANTS ARE THE

C AMPIITUDE IN COUNTS OF A SINUSOID

C AT THE PRIMARY REFERENCE PERIOD

C (1 SEC FOR SHORT PERIOD AND 25 FOR

C IONG PERIOD).

C GETCAL AISO RETURNS IN LINSTR

C THE TYPE OF INSTRUMENT,

C 1 FOR SHORT PERIOD,

C \& FOR LONG PERIOD, AND

C 3 FOR INTERMEDIATE PERIOD DATA.

C

C

C CALIS:

C FFIN

C GETLIN

C GETSTR

C

C PROGRAMMED GY MADELETINE ZIRBES

C SEPTEMBER 15, 1980

C

C DATA RECORD - INPUT

INTEGER IDATA(1)

C SET TO HOLD 16000 BITS OF TAPE INPUT RECORD

C NUMRER OF CHANNETS - OUTPUT

INTEGER ICHAN

C CAIIPRATION CONSTANT - OUTPUT REEAL CAL(3)

C INSTRUMENT

c INTEGER IINSTR

INTEGER KUC, KUI, KUL, KUS

DNTEGER I

INTEGERR LINE

INTEGER $\mathrm{K}$

INTEGER IFL, M

DNTEGER IBUFF(80)

INTEGER CODE

C FUNCTION

REAL FFIN

c

DATA KUC, KUI, KUL, KUS /'C', 'T', 'L', 'S'/

C GET NUMBER OF CHANNETS

C

CALL GETINN (DATA, 1, IBUFF)

ICHAN $=\operatorname{INT}($ FFIN $(13,60, \operatorname{IBUFF}, \mathrm{K}$, IFL $))$

IF(.NOT.(.NOT.((ICHAN .GT. O) .AND. (IFL .EQ. KUC))))GOTO 23000 GETCAL $\approx(0)$

RETURN

C

C DETERINNE TYPE OF INSTRUMENT

C

23000 CONTINUE

CALI GETSIR (28, 28, IBUFF, M, IFL, I, 1, CODE)

IF(.NOT.(CODE .EQ. KUS))GOTO 23002

IINSTR $=1$

C HSE

23002 CONTINUE 


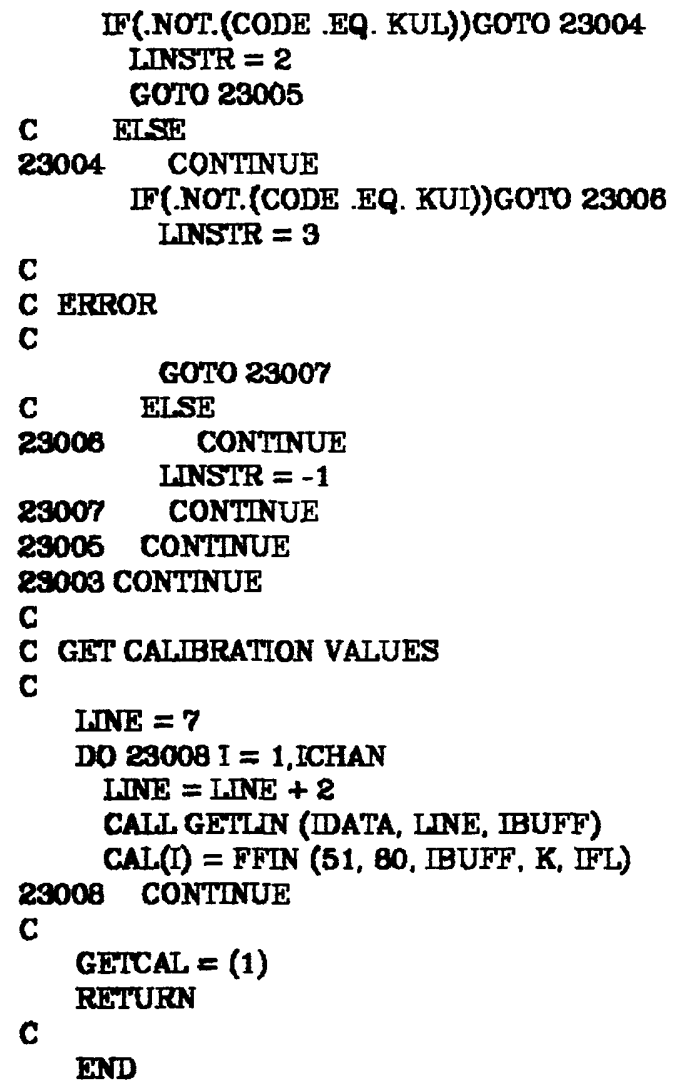


C

SUBROUTINE GETCPZ(DATA, ICHAN, AO, NP, POLES, NZ, ZEROES)

C GETCPZ SCANS DATA LOG 2 FOR THE

C CONSTANT(S), POLES AND ZEROES

C OF THE INSTRUMENT TRANSFER

C FUNCTION(S).

C

C

C CAIIS:

C FFTN

c GETIN

c

C PROGRAMMED BY MADELEINE ZIRBES

C SEPTEMBER 15, 1880

C CHARACTER BUFFER - INPUT INTEGER DATA(1)

C SET TO HOLD 16000 BIIS OF TAPE RECORD INPUT

C NUMBER OF CHANNEIS - INPUT INTEGER ICHAN

C CONSTANT - OUTPUT

REAL AO(9)

C NUMBER OF POLES - OUTPUT

INTEGER NP

C POLES - OUTPUT

COMPLEX POLES $(20,3)$

C NUMBER OF ZEROES - OUTPUT INTEGER NZ

C ZEROES - OUTPUT

C COMPLEX ZEROES(10,3)

INTEGER KUA, KUP, KUS, KUZ

INTEGER I, J, M, K, L, MR

C CHARACTER BUFFER

INTEGER IBUFF(BO)

DNTEGER LINE

INTHGER IFL

DNTEGER ITT

C FUNCTION

FOEAL FFIN

FIFAL A(6)

C

DATA KUA, KUP, KUS, KUZ /'A', 'P', 'S', 'Z'/

c ImTtalaze values

C

$\mathrm{AO}(1)=0.0$

$\Lambda 0(2)=0.0$

$\mathbf{A O}(3)=0.0$

C

$\mathrm{L}=$ QगCHAN

C CONSTANT

C

C DETERYINE WHAT LINE TO START READING THE CONSTANTS AND

C FHERE ON THAT LNNE TO BEGIN

C

IDNE $=1$

C FOR

28000 CONTINUE

CALL GETLIN (IDATA, INNE, IBUFF)

$R=\operatorname{INT}($ FFTN $(1,80$, IBUFF,, IFL $))$

IF(.NOT.(IFL .EQ. KUA))GOTO 23009

$\mathbf{M}=\mathbf{M}+\mathbf{2}$ 


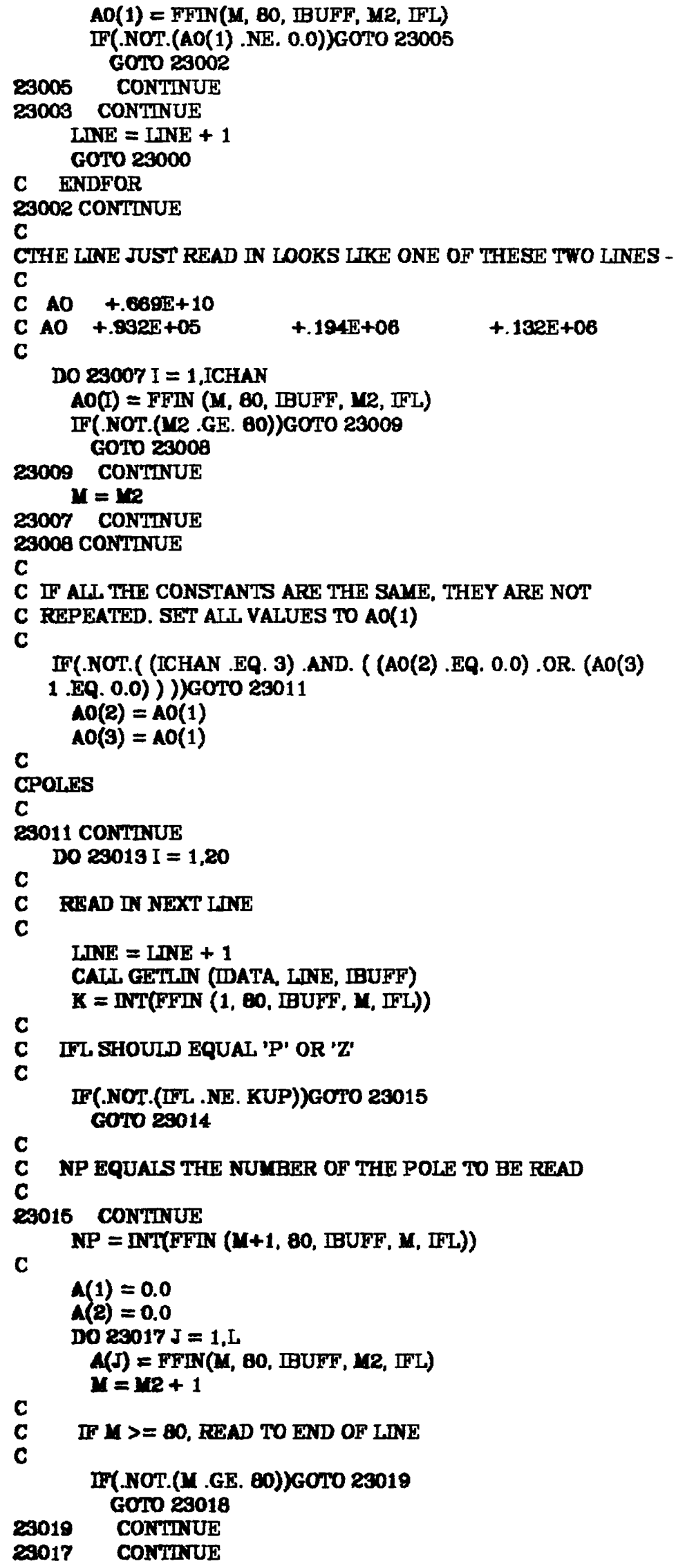




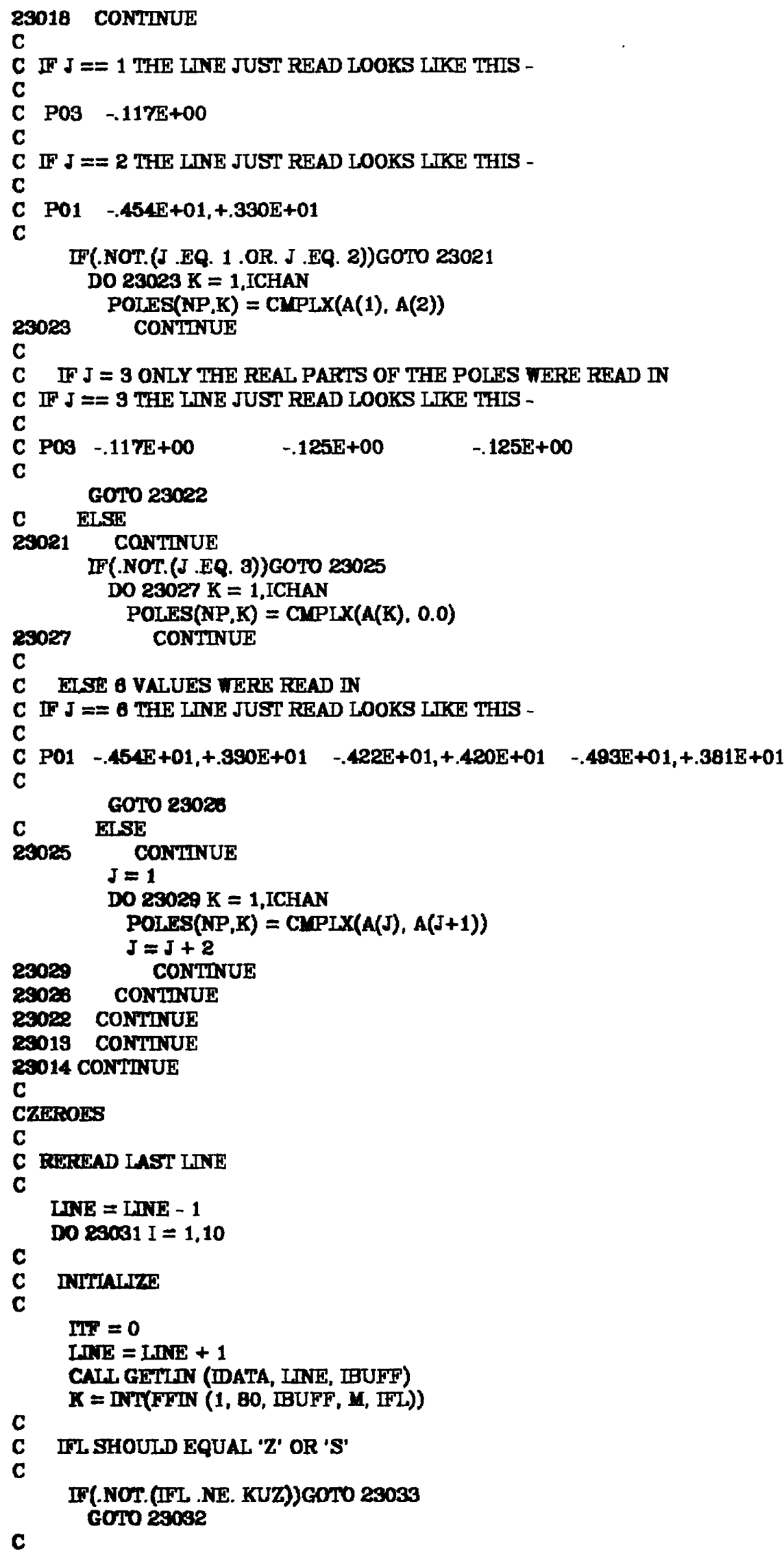




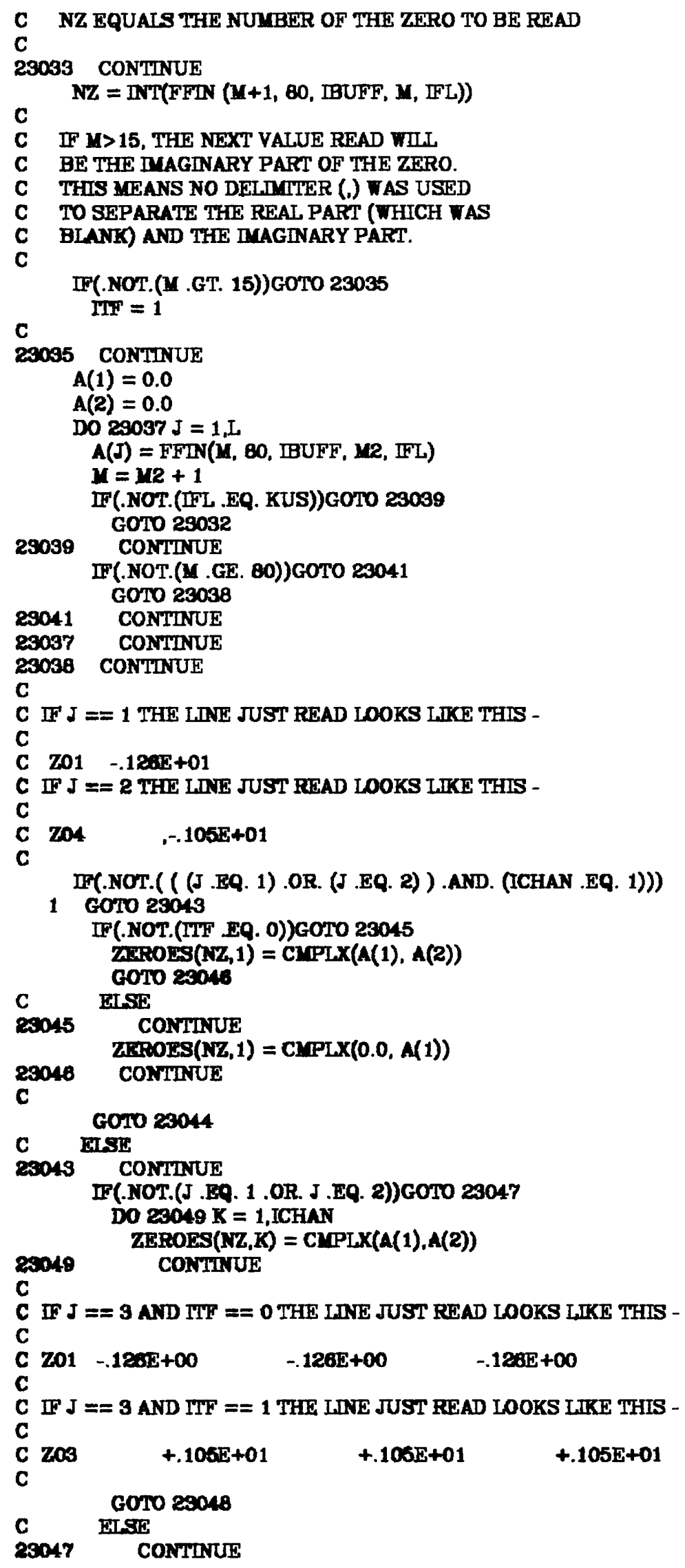




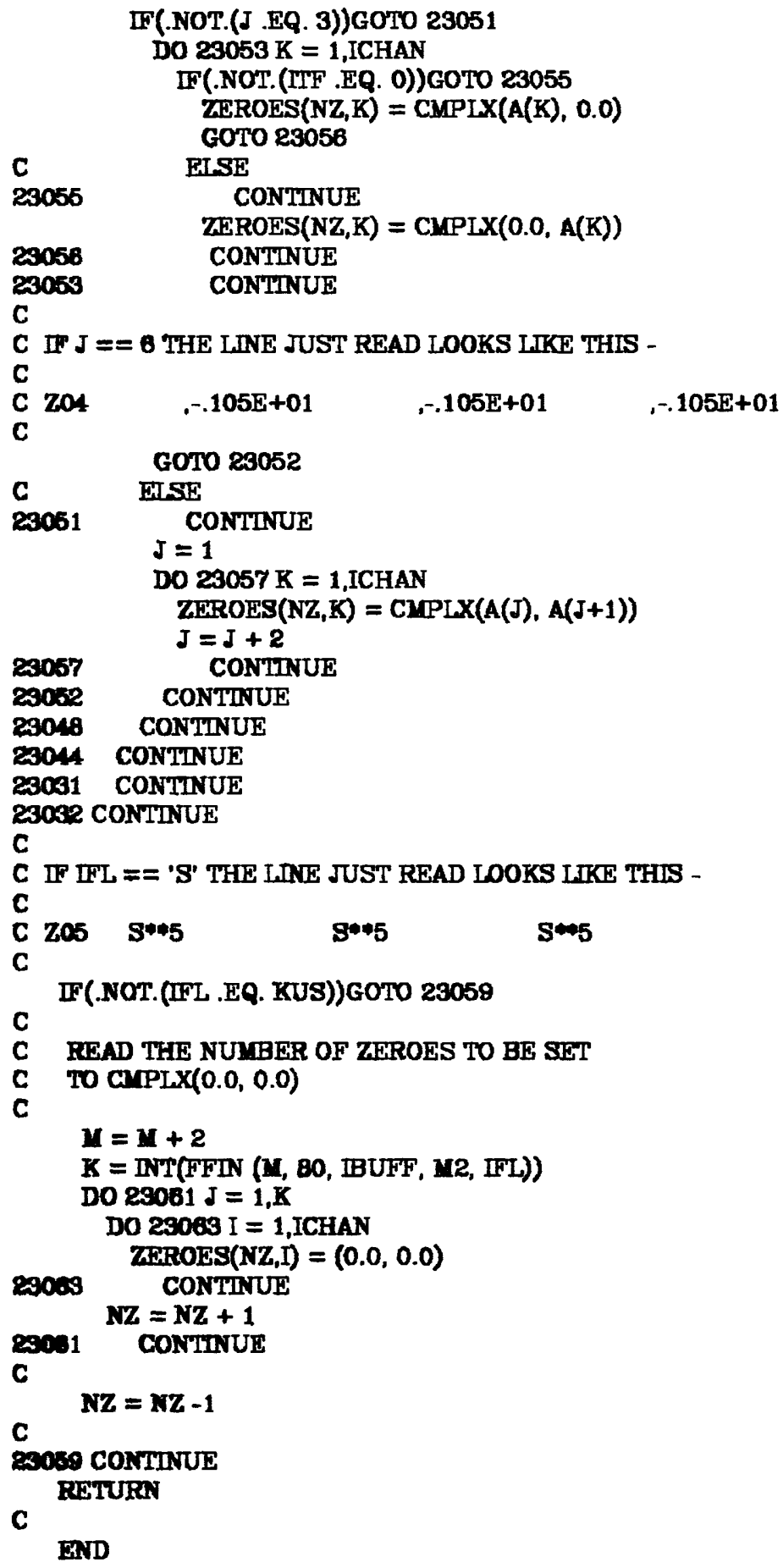


REQ.FORTRAN

C

INTEGER FUNCTION REQ(CODE, INDX, RSTART)

C FUNCTION REQ LOOPS THROUGH THE REQUESTS

C AND CHECKS TO SEE IF A REQUEST CAN BE

C FIILED BY DATA IN THIS RECORD. REQ RETURNS

C 1 IF A REQUEST CAN BE SATTSFIED, 0 OTHERWISE.

c

C

C CAIIS:

C DOY

C ISTREQ

C

C PROGRAMMED BY MADELEINE ZIRBES

C SEPTEMBER 15, 1880

C

C STATTON CODE - INPUT

INTTEGER CODE(4)

C REQUEST INDEX - OUTPUT

INTEGER INDX

C STARTING DATA POINT IN RECORD - OUTPUT

C

REAL RSTART

C

C TAPE HEADER INFORMATTON

C

C STATTON ID

INTEGER ID

C NSSTRUMENT

INTEGER INSTR

C YEAR

INTEGGER YEAR

C DAY OF YEAR

INTEGER DOFY

C HOUR

INTEGER HOUR

C NINUTE

INTEGER IIN

C SECOND

INTEGERR SEC

C MIIIISECONDS

REEAL MSS

C FORMAT ID

INTEGER NFORM

C NUNGER OF CHANNELS

INTEGER NCHAN

C NUIBER OF SANPLES

INTEGER NSAMP

C SAYPLING RATE

C

REAL RATE

C REQUEST INFORMATION

C 50 - MAXIMUM NUMBER OF REQUESTS ALLOWED

C

C REQUEST INFORMATION

C NUMBER OF REQUESTS INTEGER NREQ

C STATION CODE - CHARACTER STRING INTEGER RCODE $(50,4)$

C INSTRTUMENT ID INTEGER RINSTR(50)

C YEAR

DNTHGER RTEAR(50) 


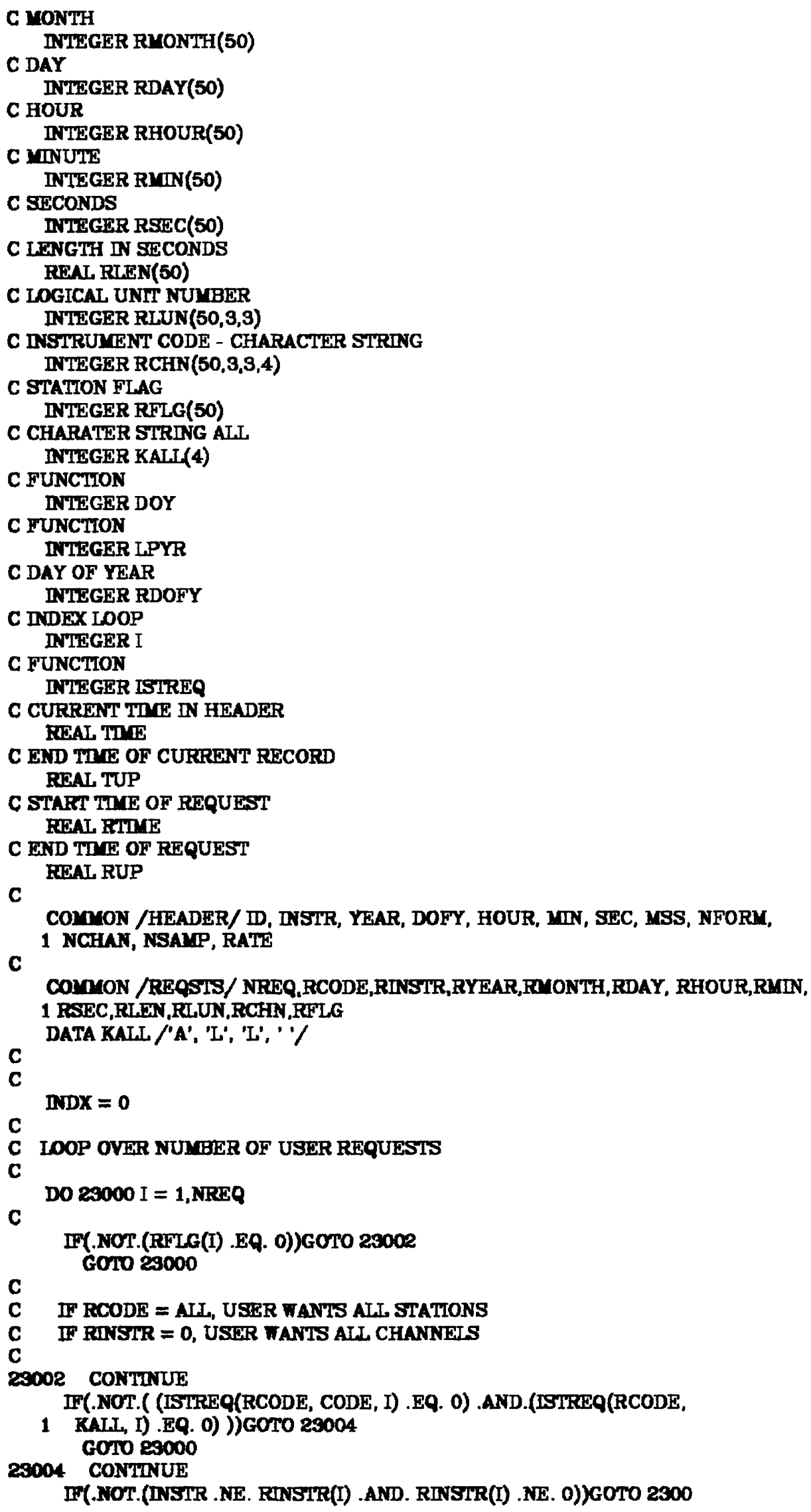




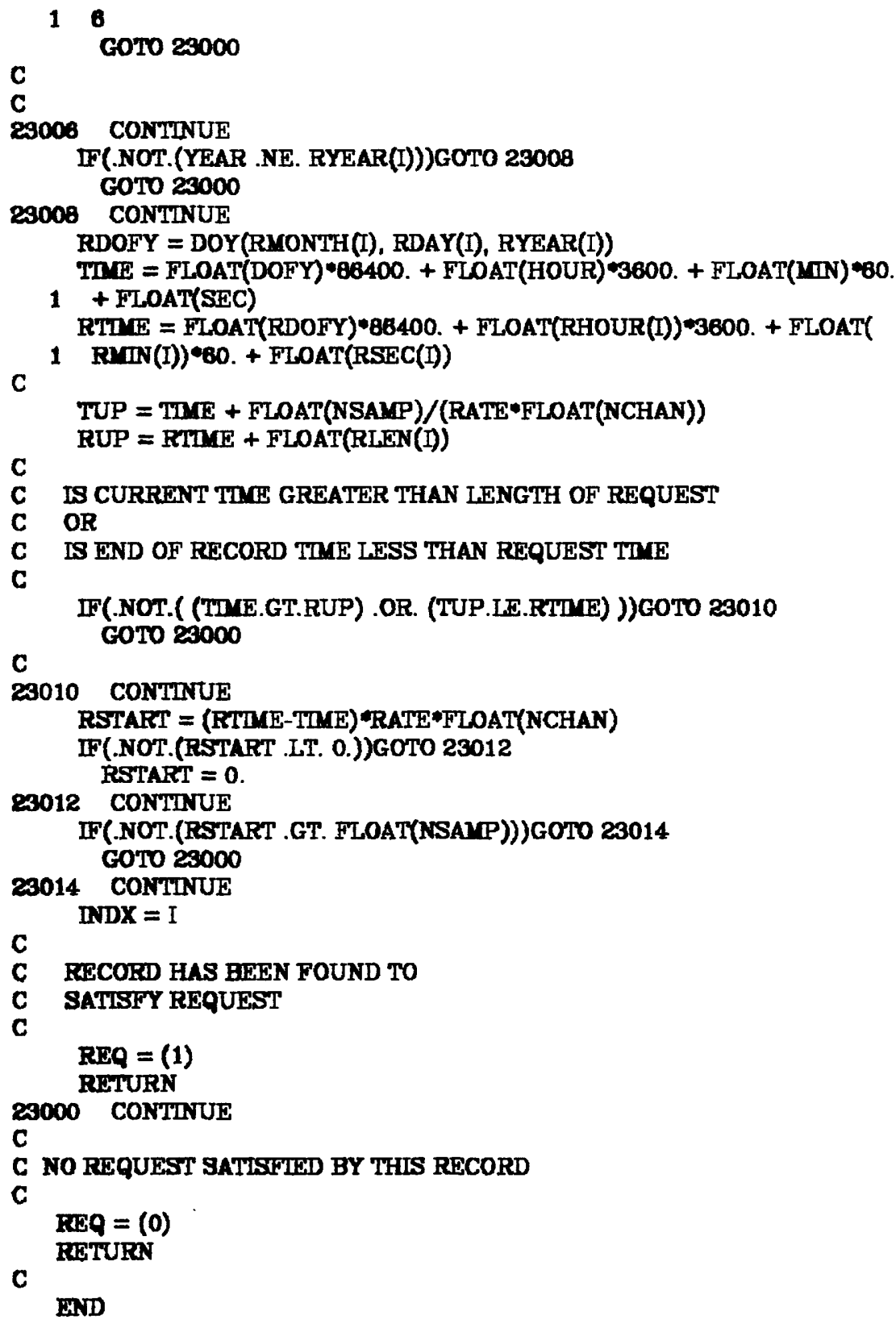


FILREQ.FORTRAN

C

SUBROUTINE FIIREQ(DR,INDX,RSTART,RDFLAG,IDATA,IERR)

C A RECORD SATISFYTNG A REQUEST HAS BEEN FOUND.

C FIIREQ FIISS THIS REQUEST.

C

C

C CALIS:

C DTPFIT

C FLSBUF

C GBYTES - USER SUPPLIED

C HEAD

C MAGRD - USER SUPPIIED

C PRHEAD

C STNDRD

C TSET

C

C PROGRAMNDD BY MADELEINE ZIRBES

C SEPTEYBER 15, 1980

C

C TAPE DRIVE LOGICAL UNIT - INPUT INTEGER DR

C INDEX OF REQUEST - INPUT INTEGER INDX

C STARTING DATA POINT IN RECORD - INPUT REAL RSTART

C READING FIAG - OUTPUT INIEEGER RDFTAG

C DATA RECORD - INPUT INIEGER IDATA(1)

C SIET TO HOLD 16000 BITS OF TAPE INPUT RECORD

C ERROR CODE

C IIIGGER IERR

C

C TAPE HEADER INFORMATHON

C

C STATION ID INTHGER II

C INSTRUMEANT INIEGER INSTR

C YEAR INTEGER YEAR

C DAY OF YEAR INTEGER DOFY

$C$ HOUR INTEGER HOUR

C IIITIS INTEGER IIN

C SECOND IVIEGER STC

C MIIJSECONDS RERAL YSS

C FORIAT ID INTEGER NFORM

C NUI BBER OF CHANNELS INTEGER NCHAN

C NUMBER OF SAMPLES INTEGER NSAMP

C SAIPLING RATE EREAI RATE

C

C RRQUEST INFORYATION 
C 50 - MAXTMUY NUMBER OF REQUESTS ALLOWED

C

C REQUEST TNFORMATION

C NUMBER OF REQUESTS INTEGER NREQ

C STATION CODE - CHARACTER STRING INTEGER RCODE $(50,4)$

C INSTRUMENT ID INTEGER RINSTR(50)

C YEAR INTECER RYEAR(50)

C MONTH

C DAY INTEGER RMONTH(50) INTEGER RDAY(50)

C HOUR INTEGER RHOUR(50)

C MNUTE INTEGER RMTN(50)

C SECONDS INTEGER RSEC(50)

C INNGIH IN SECONDS REAL RLEN(50)

C IOGTCAL UNIT NUMBER INTEGER RLUN $(50,3,3)$

C IISTRUMMNT CODE - CHARACTER STRING INTEGER RCHN $(50,3,3,4)$

C STATION FLAG

C NTIEGER RTLG(50)

C STATION INFORMATION

C

C STATION CODE - CHARACTER STRING INTEGER CODE(4)

C NUNGER OF POLES, 20 LARGEST NUMBER OF SRO POLES INTEGER NP

C NUIGER OF ZEROES, 10 LARGEST NUMGER OF SRO ZEROES INIEGER NZ

C NUNGER OF CHANNEIS INTEGER ICHAN

C CONSTANT RTSAL $10(3)$

C CAIIBRATION CONSTANT RTAL CAL(3)

C STATION ELEVATION REAL ETIVV

C STATION LATITUDE RTRAL LAT

C STATION LONGTIUDE REAL ION

C POLES COUPINXX POLES $(20,3)$

C ZREROES

c COMPLEX ZEROES(10,3)

C SMAT TIE OF DATA

C

C YGAR INTEGER SYEAR

C DAY OF YEAR INTEGER SDOFY

C HOUR TNTEGER SHOUR

C MTUTES NTHEGER ST IN 


\section{SECONDS}

C

REAL SSECS

C 500 - BUFFER SIZE OF DATA RECORD ON DISK

c

C VERTICAL BUFFER

REAL Z(500)

C NORTH-SOUTH BUFFER

REAL N(500)

C EAST-WEST BUFFER

REAL E(500)

C NUMBER OF POINTS IN BUFFERS REAL SPTS

C TOTAL NUMGER OF POINTS READ TO FILL REQUEST REAL PIS

C LOOP LIMITS

INTEGER N1,N2

C EXPECTED TINE OF NEXT RECORD

C YEAR

INTEGER UYEAR

C DAY OF YEAR INTEGER UDOFY

C HOUR INTEGER UHOUR

C MINUTE INTEGER UMIN

C SECONDS INTEGER USEC

C MIIJ.ISECONDS REAL UMS

C FUNCTION INTEGER MAGRD

C SECONDS INTEGER SSEC

C NUMBER OF FRAMES OF DATA TO READ INTIGER NWORDS

C NUNBER OF FRAYES READ INTEGER NR

C SIEA OF 980 DETERITNED BY DATA RECORD ON TAPE

C MANTISSA INTEGER MANT(980)

C CHARACTERISTHC INIEGER NCHAR(990)

C LOOP INDEX INIEGER I

C INT OF SPIS INIEGER IPIS

C FUNCTION REAL DTPFIT

C MIIIJISECONDS ERAT FMS

C MIILISECONDS REAL SUS

C TOTAL NUYBER OF PONNTS TO EXIRACT RTAL TPIS

C COMMON /HEADER/ ID, INSTR, YEAR, DOFY, HOUR, MIN, SEC, MSS, NFORM,

C 1 NCHAN, NSAMP, RATE

COMMON /REQSTS/ NREQ,RCODE,RINSTR,RYEAR,RYONTH,RDAY, RHOUR,RMIN,

c 1 RSEC,RLEN,RLUN,RCHN,RFLG

COMMON /STINFO/CODE, ICHAN, NP, NZ, IAT, LON, ELEV, AO, CAL,

c 1 POLES, zarotes 
CONMON /SSTART/ SYEAR, SDOFY, SHOUR, SMIN, SSECS

C

ON /BUFFTER/Z, N, E, SPTS, PIS

DATA NWORDS/1000/

C

CALL TSETY(SYEAR, SDOFY,SHOUR,SMIN,SSEC,SUS)

FMS $=($ RSTART 1000.$) /($ RATE*FLOAT(NCHAN) $)$

SMS = SMS + FMS

CALI. STNDRD(SYEAR,SDOFY,SHOUR,SMIN,SSEC,SYS)

C

SSECS = FLOAT(SSEC) + SMS $/ 1000.0$

C

SPTS $=0$

C PRINT FIIE HEADER

C

C

CAIL PRHEAD(RL,UN,RCHN,INDX,INSTR)

C TPTS - TOTAL NUMBER OF POINTS TO RETRIEVE

C TO SATISFY REQUEST

C

TPTS = RATE $*$ FLAT(NCHAN) *RLEN(TNDX)

TPTS = RSTART + TPTS

C

N1 $=$ INT(RSTART) +1

C SET EXPECTED TLE OF NEXT RECORD TO BE READ

C

CALL TSET(UYEAR, UDOFY, UHOUR, UMTN,USEC,UMS)

FMS = FLOAT(NSAMP) *1000./(RATE*FLOAT(NCHAN))

UIS = UMS + FMS

C

CALC STMDRD(UYEAR,UDOFY,UHOUR,UMIN,USEC, UMS)

C LOOP

C KGEP READING AND DECODING BNNARY DATA UNTLL

C 1.) REQUEST IS SATISFIED

C 2.) END OF FIIE

C 3.) ERROR

C 4.) NEXT RECORD IS NOT CONTHNUOUS

C

C FOR

29000 CONTLUUE

IF(.NOT.(NFORM.EQ.1))GOTO 23003

C SRO FORUAT

CALI GBYTES(IDATA, MANT, 164, 12,4,NSAMP)

CALL GBYTES(IDATA, NCHAR, 160,4,12,NSAMP) GOTO 28004

C ELSE

28003 CONTINUE

IF(.NOT.(NFORM.EQ.2)) GOTO 23005

C DISSN FORMAT

C CAIL GBYLES(IDATA,MANT, 160,16,0,NSANP)

23005 CONTINUE

23004 CONTINUE

IF(.NOT.(IPIS .GT. FLOAT(NSAYP)))GOTO 23007 NR $=$ MSAP GOTO 23008

C RISTE

2s007 CONTINUE

$\mathrm{NR}=\mathrm{INT}(\mathrm{TPTS})$

8s008 CONTINUE

C

C DECODE BINARY DATA AND FTLL BUFFERS

C

DO 23009 $\mathrm{I}=\mathrm{N} 1, \mathrm{NR}, \mathrm{NCHAN}$ 


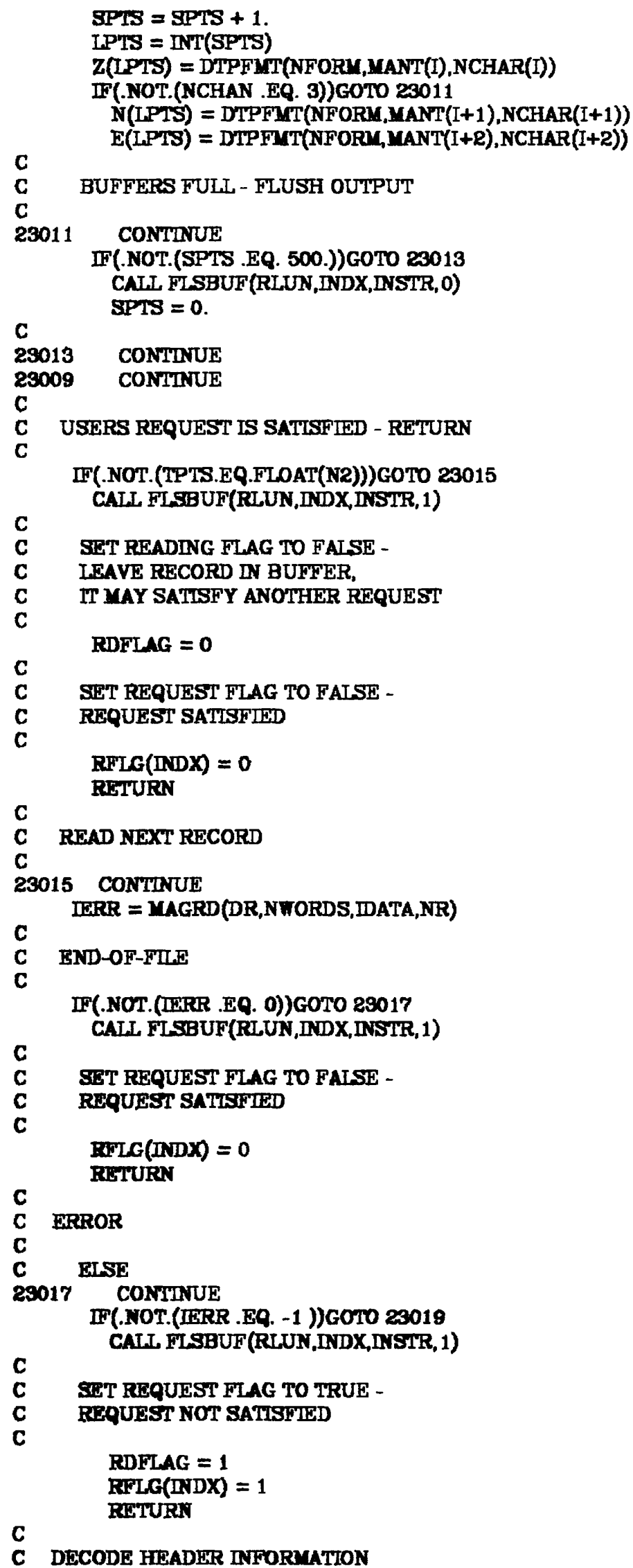

C

C DTCODE HEADHR INFORMATION 


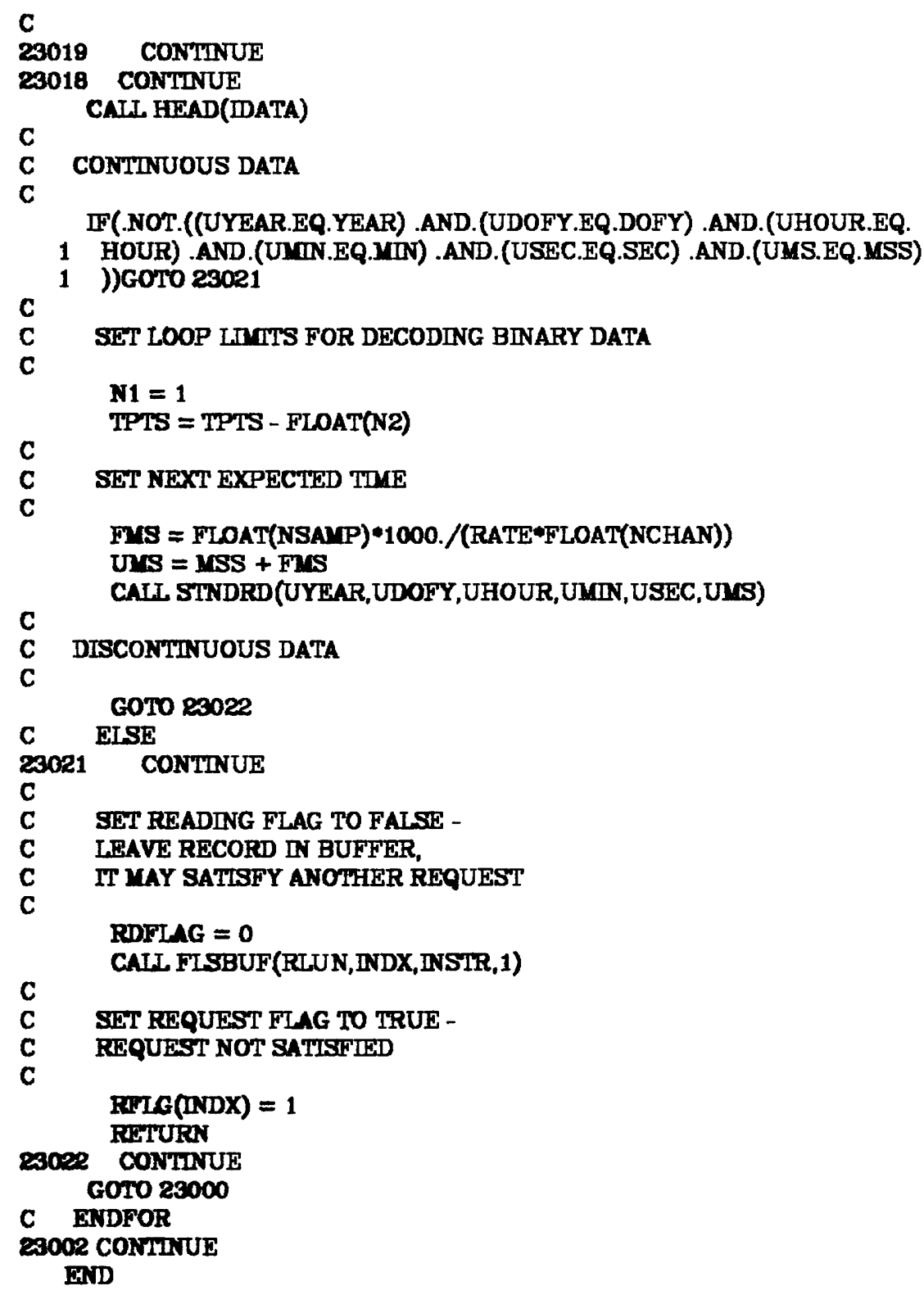


PRHEAD.FORTRAN

C

SUBROUTINE PRHEAD(FID,CHAN,I,M)

C PRHEAD WRITES A HEADER RECORD TO

C THE APPROPRIATE OUTPUT FILES.

C

C

C CALIS NO OTHER ROUTINE.

C

C PROGRAMMED BY MADEIEINE ZIRBES

C SEPTEMBER 15, 1880

C

C LOGICAL UNIT NUMBER - INPUT

INTEGER FID $(50,3,3)$

C INSTRUUENT CODE - INPUT

INTEGER CHAN $(50,3,3,4)$

C REQUEST INDEX - INPUT

TNTEGER L

C INSTRUNENT INDEX - INPUT

C

INTEGER $\mathbf{M}$

C

C STATION INFORMATION

C

C STATION CODE - CHARACTER STRING

INTEGER CODE(4)

C NUMBER OF POLES, 2O LARGEST NUMBER OF SRO POLES THIEGER NP

C NUMBER OF ZEROES, 10 LARGEST NUMEER OF SRO ZEROES DITEGER NZ

C NUMBER OF CHANNEIS INTEGER ICHAN

C CONSTANT RTRAL AO(B)

C CALIBRATHON CONSTANT FESAL CAL(3)

C STATION ETLFVATON

RJAL EREV

C STATION LATTUDE BISAL IAT

C STATION LONGITUDE RIRAL ION

C POLES

CONTPIEX POLES $(20,3)$

C TAROEES

c

COI PLEX ZEROES(10,3)

C START TIME OF DATA

C

C YEAR

INITEGER SYEAR

C DAY OF YTAR

INITEGER SDOFY

C HOUR

IMTEGER SHOUR

C IINUTES

INTEGER SIIN

C STCONDS

RREAL SSECS

C

C TAPE HEADER INFORMATTON

C

C STATHON ID 


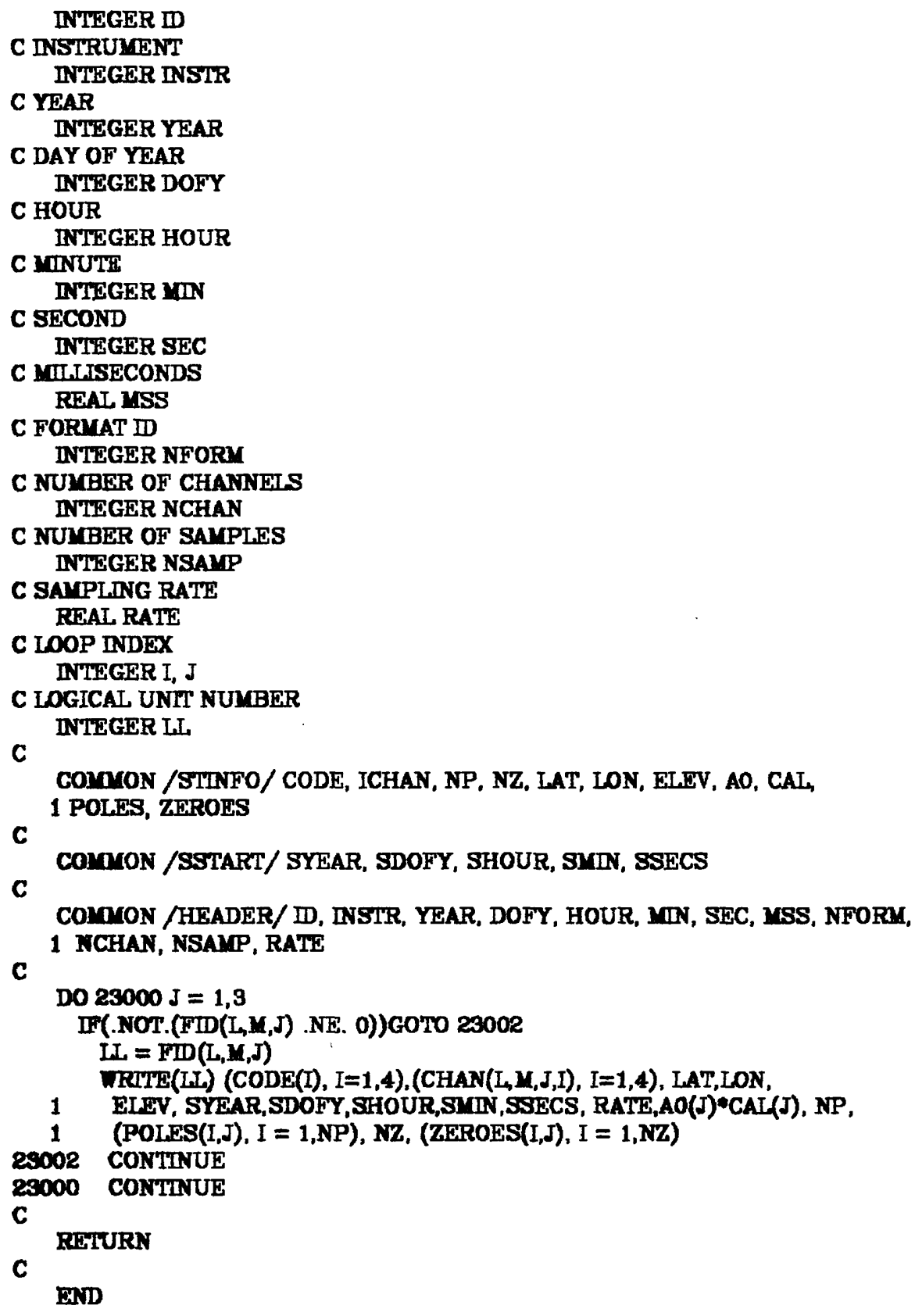


HLSBUF.FORTRAN

c

SUBROUITINE FISBUF(FID, L, M, ITF)

C FISBUF WRITES DATA BUFFERS TO THE

C APPROPRIATE OUTPUT FIIES.

C

C

C CALIS NO OTHER ROUTHE.

c

C PROGRAMMED BY MADELEEINE ZIRBES

C SEPTEMBER 15, 1980

c

C IOGICAT UNIT NUMBER - INPUT

INTEGER FTD $(50,3,3)$

C REQUEST INDEX - INPUT

INTEGER L

C INSTRUMENT INDEX - INPUT

INTEGER $M$

C DATA FIAG

c

INTEGER IHF

C

C 600 - BUFFER SIZE OF DATA RECORD ON DISK

c

C VERTHCAL BUFFER

REAL Z(500)

C NORTH-SOUTH BUFFER

REAL $N(500)$

C EAST-WEST BUFFER

REAL E(600)

C NUMBER OF POINTS IN BUFFETS

REAL SPIS

C TOTAL NUMBER OF POINTS READ TO FUL REQUEST

RTAL PIS

TNTEGER I

C LOGICAI UNIT NUYBER

INTEGER II

REAL, BIG

IEAL ONE

COMMON / BUFFER/ Z, N, E, SPTS, PIS

DATA BIG $/ 10000000000$.

c

DATA ONE /1./

C TOTAT NUMBER OF DATA VALUES WRITIEN TO OUTPUT FILES

C

C

PIS = PIS + SPIS

C

C IF MFF $=1$, THIS IS THE IAST DATA RECORD TO

C BE WRITEN FOR THIS REQUEST

c

C VERTTCAL BUFFER

C

IF (.NOT. (FID(L,M,1) .NE. O))GOTO 29000

IN $=\mathrm{FTD}(\mathrm{L}, \mathrm{M}, 1)$

WRITE(LL) SPIS, (Z(I), I=1,SPIS)

IF(.NOT.(IIF .EQ. 1))GOTO 23002

exO0R CONTINUE

C

C NORTH/SOUTH BUFFER

C

20000 CONTINUE 
IF(.NOT.(FTD(L,M,2) .NE. 0))GOTO 23004 $L L=F I D(L, M, 2)$

WRITE(II) SPTS, (N(I), I=1,SPTS)

IF(.NOT.(ITF .EQ. 1))GOTO 23006

WRITE(LL) ONE, BIG

23006 CONIINUE

C

c EAST/WEST BUFFER

C

23004 CONTINUE

IF(.NOT.(FID(L,M,3) .NE. 0))GOTO 23008

$\mathrm{LLL}=\mathrm{FI}\left(\mathrm{I}_{2}, \mathbf{M}, 3\right)$

WRTTE(LL) SPTS, (E(I), I=1,SPTS)

IF(.NOT.(ITF .EQ. 1))GOTO 23010

WRITE(LL) ONE, BIG

23010 CONTINUE

C

C

23008 CONTINUE

REETURA

C

END 
TIME HANDLING ROUTINES

C

SUBROUTINE TSET(SYEAR, SDOFY, SHOUR, SIIN, SSEC, SMS)

C ISET SETS THE TIME VARIABLES EQUAL TO THOSE

C IN COMMON BLOCK /HEAD/

C

C

C CAIJS NO OTHER ROUTINE.

C

C PROGRAMMED BY MADELEINE ZIRBES

C SEPTEMBER 15, 1980

C

C YEAR - OUTPUT INTEGER SYEAR

C DAY OF YEAR - OUTPUT INTWGER SDOFY

C HOUR - OUTPUT INTEER SHOUR

C MINUTE - OUTPUT INTEGER SITN

C SECONDS - OUTPUT INTEGER SSEC

C MIILISECONDS - OUTPUT

C REAL BMS

C

C TAPE HEADER INFORMATTON

C

C STATON ID INTEGER ID

C INSTRUNIENT INILGER INSIR

C YEAR ITILGER YEAR

C DAY OF YEAR ITIEGER DOFY

C HOUR INTEGER HOUR

C IITUTE INIIEGER MIN

C EgCOND

INIEGER SEC

C MIIJSECONDS FTEL YSS

C FORUAT ID ITIEGER NFORA

C NUM BERR OF CHANNETS INTEGER NCHAN

C NUNGER OF SAMPLES TITEGER NSAMP

C SAYPINIG RATE RTRAL RATE

c COMON /HEADER/ ID, INSTR, YEAR, DOFY, HOUR, IIN, SEC, MSS, NFORA,

C 1 NCHAN, NSAYP, RATE

SYEAR = YEAR

SDOFY = DOFY

SHOUR = HOUR

STIN $=$ YIN

SSEC $=\operatorname{SECC}$ 
$-161-$

SMS $=$ uSS

C RETURN

C END 
STWDRD.FORTRAN

C

SUEROUTINE STNDRD(YEAR, DOFY, HOUR, MINUTE, SEC, MS)

C STIDRD CHECKS TO SEE THAT THE

C TINE VALUES ARE WTHHN LEGAL IDMISS.

C

C

C CAIIS:

C IPYR

C

C PROGRAYMED BY YADEJEINE ZIRBES

C SEPTEMBER 15, 1890

C

C YRAR - INPUT/OUTPUT

INTEGER YEAR

C DAY OF YEAR - INPUT/OUTPUT

INTEGER DOFY

C HOUR - INPUT/OUTPUT INTEGER HOUR

C MINUTE - INPUT/OUTPUT IIIEGER ITNUTE

C SECOND - INPUT/OUTPUT TIIBGER SEC

C MIILISECONDS - INPUT/OUTPUT

C REAL MS

C FUNCHON

INIEGER LPYR

C

C FOR

28000 CONTTNUE

IF(.NOT.((IS .GE. 1000.) .OR.(SEC .GE. 60) .OR.(MINUTE .GE. 60)

1 .OR.(HOUR .GE. 24) .OR.((IPYR(YEAR) .EQ. 1) .AND. (DOFY .GT.

1 303)) .OR.((LPYR(YEAR) .EQ. 0) .AND. (DOFY .GT. 385)) ))GOTO 2

13003

c

IF(.NOT.(MS .GE. 1000.))GOTO 23005

$\operatorname{Stg}=\sin C+1$

IS $=1 S-1000$

29005 CONTINUE

IF(.MOT.(SEC .GE. 60))COTO 29007

IINUTE $=$ IINUTE +1

SARC $=$ SEC -6

28007 CONTINUE

IF(.NOT.(NINUTE .GE. 60))GOTO 23000

HOUR $=$ HOUR +1

MINUTE $=$ MINUTE -60

20009 CONTWUE

IF(NOT.(HOUR .GE. 2A))GOTO 29011

DOFY $=$ DOFY + 1

HOUR = HOUR -24

20011 CONTINUE

IF(.NOT.(IPYR(YEAR) .EQ. 1 .AND. (DOFY .GT. 363))) GOTO 23013

$\operatorname{MTAR}=\operatorname{YEAR}+1$

DOFY $=$ DOHY -300

C

RoO13 CONMTUE

IF(.NOT.(IPYR(YEAR) .EQ. O .AND. (DOFY .GT. 365))) GOTO 23015

YEAR = YEAR + 1

C

DOFY $=$ DOFY -305

P8015 CONMTUE

GOTO 20004 
C EISE

23003 CONTINUE

GOTO 23002

29004 CONTINUE

GOTO 23000

C ENDFOR

23002 CONTINUE

RETURN

C

END 
DAYMO.FORTRAN

C

INTEGER FUNCTION DAYMO (DOFY, MONTH, DAY, YEAR)

C FUNCTION DAYMO DETERMINES THE MONTH AND DAY

C OF THE MONTH, GIVEN THE YEAR AND DAY OF YEAR.

C IT RETURNS 1 IF IT WAS SUCCESSFUL, O OTHERWISE.

C IF DOFY IS NOT WTTHNN LEGAL IMTTS, MONTH AND

C DAY WIIL BE RETURNED AS ZERO.

C

c

C CALIS:

C LPYR

c

C PROGRAMMED BY MADELEINE ZIRBES

C SIFPTEMBER 15, 1980

C

C DAY OF YEAR - INPUT

INTEGER DOFY

C MONTH - OUTPUT

DNTEGER MONTH

C DAY OF YONTH - OUTPUT

INTEGER DAY

C YEAR - INPUT

INTEGER YEAR

C

C DAY OF YEAR

INTEGER DAY

C FUNCTION

INTEGER IPYR

C NUSGER OF DAYS IN MONTH

INTEGER MDAYS(12)

C

DATA MDAYS/31, 28,31,30,31,30,31,31,30,31,30,31/

DAY $=$ DOFY

IF(.NOT.(IDAY.LT.1))GOTO 23000

MONTH $=0$

DAY $=0$

DAYMO $=(0)$

RETURY

C

89000 CONTINUE

IF(.NOT.(IPYR(YEAR) .EQ. 1))GOTO 23002

$\operatorname{MAAYS}(2)=29$

C सHSE

coro 23003

23002 CONTINUE

$\operatorname{MDAYS}(2)=28$

23003 CONTINUE

C

DO 23004 YONTH $=1,12$

DAY $=$ IDAY

IDAY = DAY - MDAYS(MONTH)

IF(.NOT.(IDAY.LE.O)) GOTO 23006

DAYMO $=(1)$

REETURA

29003 CONTTNUE

23004 CONTINUE

C

MONTH $=0$

DAY $=0$

DAYMO $=(0)$

RETUUR

C 
END

- 165 - 
DOY.FORTRAN

C

INTEGER FUNCTION DOY (MONTH, DAY, YEAR)

C FUNCTION DOY DETERIINES THE DAY OF THE

C YEAR, GIVEN THE MONTH, DA AD YEAR.

C IF MONTH OR DAY ARE IILEGAL, THE RETURN

C VALUE OF THE FUNCTION IS ZERO.

C

C

C CALLS:

C IPYR

C

c PROGRAMMED BY YADELEINE ZIRBES

C SEPTEUBER 15, 1980

C

C MONTH - INPUT

INTEGER MONTH

C DAY OF MONTH - INPUT

INTEGER DAY

C YEAR - INPUT

INTEGER YEAR

C FUNCTTON

INTEGER LPYR

INTEGER INC

INTEGER NDAYS(12)

DATA NDAYS $/ 0,31,59,00,120,151,181,212,243,273,304,334 /$

IF(.NOT.(MONTH .IT.1 .OR. MONTH .GT. 12))GOTO 23000 DOY $=(0)$ RETURN

85000 CONTTINE

IF(.NOT.(DAY .LT. 1 .OR. DAY .GT. 31))GOTO 23002

DOY $=(0)$

RETURN

29002 CONTINUE

IF(.NOT.(LPYR(YEAR) .EQ. 1 .AND. MONTH .GT. R))GOTO 23004 INC $=1$

C EISE

GOTO 23005

23004 CONTINUE

IITC $=0$

29005 CONTINUE

DOY $=($ NDAYS (MONTH) + DAY + INC)

RETURO

END 
IPYR.FORTRAN

C

INTEGER FUNCTION IPYR(YEAR)

C FUNCTION LPYR DETERIINES IF YEAR

C IS A LEAP YEAR.

C

C THIS FUNCTION USES THE INTRINSTC

C FUNCTION MOD. IF YOUR MACHINE

C DOES NOT SUPPLY THIS FUNCTION,

C MAKE ONE -

C $\operatorname{YOD}(I, J)=\operatorname{IABS}(I-(I / J) * J)$

C

C

C CAIIS:

C YOD - INTRINSIC FUNTION

C

C PROGRAMMED BY MADELLEINE ZIRBES

C SEPIEMBER 15, 1980

C

C YEAR - INPUT

INTEGER YEAR

IF(.NOT.(MOD(YEAR, 400) .EQ. O))GOTO 23000 IPYR $=(1)$ RETURN

23000 CONTINUE

IF(.NOT.(MOD(YEAR, 4) .NE. 0))GOTO 23002 $\mathrm{LPYR}=(0)$

RETURN

23002 CONTINUE

IP(.NOT.(MOD(YEAR, 100) .EQ. O))GOTO 23004 IPYR $=(0)$ RETURN

25004 CONTINUE

LPYR = (1)

RETTURN

END 
CHARACTER HANDIING ROUTINES

GETHN.FORTRAN

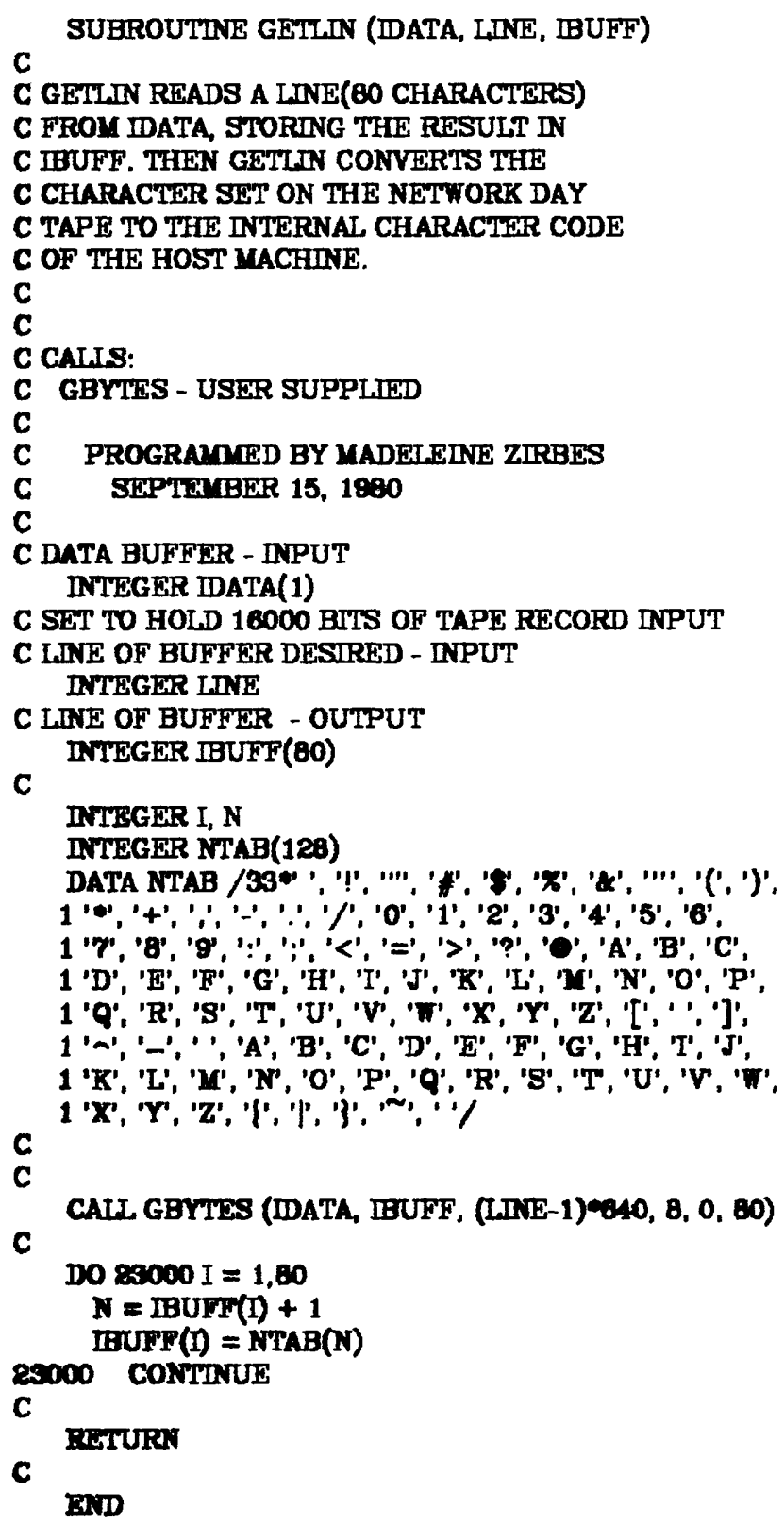


FFTN.FORTRAN

C

REAL FUNCTION FFTN(N1,N,IA,M,IFL)

C

C FUNCTION FFIN SCANS THE N CHARACTER STRING IA FOR A NUMERIC CONSTANT.

C IT WILL CORRECTLY INTERPRET A NUMBER IN E OR F FORMAT,

C AND RETURN IT AS THE FUNCTION VALUE. IFL $=$ IA(M)

C IS THE FIRST NON-BLANK CHARACTER THAT CAN'T BE A PART OF THE

C CONSTANT.

c

C

C CAIJS NO OTHER ROUTMNE.

C

C PROGRAMYED BY RAY BULAND

C AUGUST 21, 1980

C

C START INDEX - INPUT

INTEGER N1

C STOP INDEX - INPUT

INTEGER N

C CHARACTER STRING - INPUT

INTEGER IA(80)

C THE CHARACTER SIRING IS STORED IN THE USERS

C IITERANAL CHARACTER CODE, ONE CHARACTER

C (BLANK PADDED TO THE LEF'T) PER INTEGER WORD.

C INDEX IN IA OF FIRST IIJEGAL CHARACTER - OUPUT INTEGER $\boldsymbol{M}$

C IILEGAL CHARACTER - OUTPUT

C RTIEGER IFL

INTEGER IB(10)

INIEGER KBL, IPL, KON, KDP, KUE

INTEGER I, J, $\mathrm{K}$

ITHEGER MIN

INIEGER YIEX

INTEGER LFL

REAL SC1

IASAL SC2

FRAL EX

DaTA IB /'0', '1', '2', '3', '4', '5', '6', '7', '8', '0'/

DATA KBL, KTPL, KMN, KDP /' ', '+'. '-' ' '

c

DATA KUE / 'E'/

c

SET UP SOME DEFAULTS.

FFTN $=0$.

$\operatorname{sc1}=10$

$\operatorname{SCR}=1$

ITN $=1$

Ex $=0$.

c

$\operatorname{Imx}=1$

C STIP THE LEADING BLANKS.

C

$\mathrm{DO} 25000 \mathrm{~K}=\mathrm{N} 1, \mathrm{~N}$

IF(.MOT.(IA(K).NE.KBL))GOTO 23002

GO TO 10

ES00R CONTHNUE

20000 CONIINUE

co TO 80

c

c CHECK FOR UNARY PLUS.

C 
10 IFL $=\mathrm{K}$ $\mathbf{K}=\mathbf{K}+1$

GOTO 23005

C EISE

\&3004 CONTINUE

C

C CHECK FOR UNARY MENUS.

C

IF(.NOT.(IA(K).EQ.KMN))GOTO 23008

MIN $=2$

$\mathbf{K}=\mathbf{K}+1$

23006 CONTINUE

23005 CONIINUE

DO $23008 \mathrm{I}=\mathrm{K}, \mathrm{N}$

IF(.NOT.(IA(I) .NE.KBL))GOTO 23010

GOTO 29009

23010 CONTHNUE

23008 CONTINUE

23009 CONTINUE

$\mathrm{K}=\mathrm{I}$

C

C ACCUMULATE THE NUMERIC CONSTANT.

c

C

$\mathrm{DO} 22012 \mathrm{I}=\mathrm{K}, \mathrm{N}$

C CHECK FOR A DECMAL POINT.

IF(.NOT.(IA(I).NE.KDP))GOTO 23014

C

C SEE IF THE NEXT CHARACTER IS A DIGTT.

C

DO $29016 \mathrm{~J}=1,10$

IF(.NOT.(IA(I).EQ.IB(J)))GOTO 23018

GO TO 20

23018 CONTINUE

25016 CONTINUE

CO TO $\$ 0$

C

c CONSTRUCT THE CONSTANT

Co IF(.NOT.(SC1.LT.5.))GOTO 28020

$\mathrm{SC2}=\mathrm{SC2} * 1$

2000 CONTTIUE

FITI $=$ FIT $\$$ SC $1+(\mathrm{J}-1)-\mathrm{SCC} 2$

GOTO 28015

C EISTE

20014 CONTHUE

IF(.NOT.(BC1.LT.5.))GOTO 23022 GO TO 60

EAOPR CONTINUE

$\operatorname{sc1}=1$

E015 CONTINUE

DOD12 CONTINUE

GO TO 80

c

C CHECK FOR SCIENTIFIC NOTATION.

C

S0 IF(.NOT.(LFI.NE.I.AND.IA(I).EQ.KUE))GOTO 23024

$\mathrm{K}=\mathrm{I}+1$

$I F L=K$

c

C CHECK FOR UNARY PLUS IN THE EXPONENT.

C

IF(.NOT.(IA(X).EQ.KPL)) GOTO 23028 


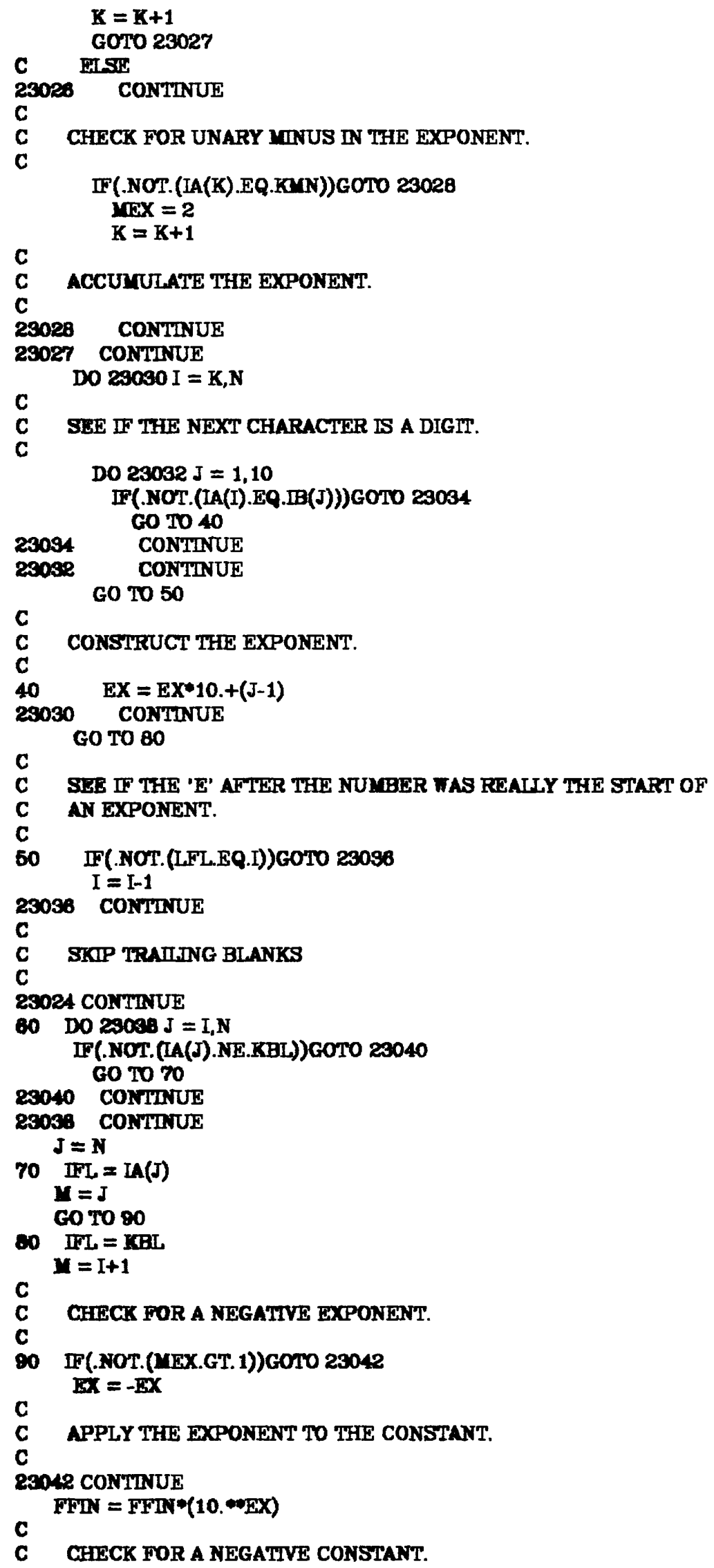

C

C SES IF THE 'E' AF"TER THE NUMGER WAS FWALLY THE START OF

C AN EXPONENT.

C

60 IF(.NOT.(LFL.EQ.I))GOTO 23038$$
\mathrm{I}=\mathrm{I}-1
$$

23038 CONITNUE

c

c

C

8S024 CONTINUE

60 DO $29083 \mathrm{~J}=\mathrm{I}, \mathrm{N}$

IF(.NOT.(IA(J).NE.KBL)) COTO 23040 GO 1070

23040 CONITNUE

89038 CONTINUE

$J=N$

70 IFL $=I A(J)$

$\mathbf{Y}=\mathrm{J}$

COTO 80

80 IFL = IBLL

c

$\mathbf{M}=\mathrm{I}+1$

C CHECK FOR A NEGATVE EXPONENT.

C

80 IF(.NOT.(MEX.GT. 1))GOTO 23042

$\mathrm{EXX}=-\mathrm{EX}$

C

C APPLY THE EXPONENT TO THE CONSTANT.

C

2QP42 CONTTNUE

c

FFIN $=$ FFIN $*(10 . * \mathrm{EX})$

C CHECK FOR A NEGATIVE CONSTANT. 
$-172-$

C

IF(.NOT.(MIN.GT.1))GOTO 23044

FFIN $=-$ FFFIN

23044 CONTINUE RETURN

C

END 


\section{GETSTR. FORTRAN}

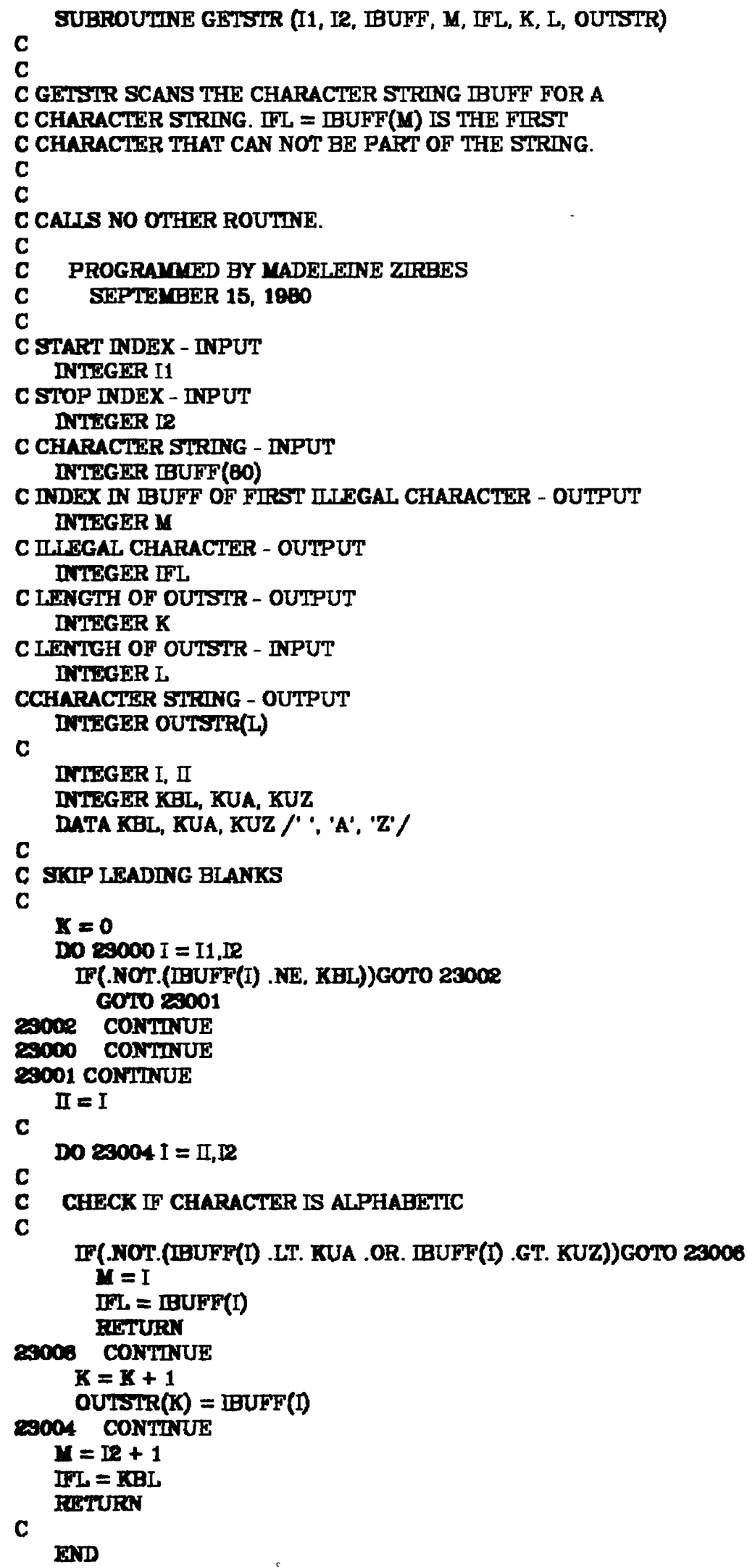

END 
ICPYCH.FORTRAN

c

SUBROUTINE ICPYCH (I1, I2, I, J, K)

C ICPYCH COPIES ARGUNENT I2 TO I1.

C IT COPIES EXACTLY 4 CHARACTERS.

C

C

C CAIJS NO OTHER ROUTINE.

C

C PROGRAMMED BY MADELEINE ZIRBES

C SEPTEMBER 15, 1880

C

C INSTRUMENT CODE - OUTPUT INTEGER I1 $(50,3,3,4)$

C INSTRUMENT CODE - INPUT INIEGER $\operatorname{I2}(3,3,4)$

C REQUEST INDEX - INPUT INTEGER I

C INSTRUNENT INDEX - INPUT INTEGER J

C CHANNEI INDEX - INPUT INTEGER $\mathrm{K}$

C

c

INTEGER L

DO $23000 \mathrm{~L}=1,4$

$\operatorname{II}(I, J, K, L)=\mathbb{R}(J, K, L)$

28000 CONTINUE

C

C

RETURN

END 
C

INTEGER FUNCTTON ISTREQ (I1, I2, I)

C ISTREQ COMPARES ARGUMENTS I1 AND I2 C AND RETURNS 1 IF THEY ARE EQUAL, C 0 OTHERWTSE.

C

C

C CAIIS NO OTHER ROUTINE.

C

C PROGRAYMED BY MADELEINE ZIRBES

C SEPTEMBER 15, 1880

C

C CHARACTER STRING - INPUT INTEGER I1 $(50,4)$

C CHARACTER STRING - INPUT INTEGER I2(4)

C ARRAY INDEX - INPUT

c

c

INTEGER I

DO $23000 \mathrm{I}=1,4$

IF(.NOT.(I1(L,I) .NE. I2(I)))GOTO 23002 ISTREQ $=(0)$

RETURN

23002 CONTINUE

23000 CONTINUE

ISTREQ $=$ (1)

RETURN

C

END 
BINARY DATA HANDLING ROUTINES

HEAD.FORTRAN

C

SUBROUTINE HEAD(IDATA)

C HEAD DECODES HEADER INFORMATION

C AND SETS APPROPRIATE VALUES IN COMMON

C BIOCK /HEADER/

C

C

C CALIS:

C GBYTES - USER SUPPLIED

C

C

PROGRAMMED BY YADELLEINE ZIRBES SKPTEMBER 15, 1860

C DATA RECORD - INPUT

INTEGER IDATA(1)

c

c

C TAPE HEADER INFORYATTON

C

C STATION ID

INTEGER ID

C INSTRUMENT

INTEGER INSTR

C YEAR

INIEGER YEAR

C DAY OF YEAR

INTEGER DOFY

C HOUR

IITSGER HOUR

C NTIUTE

INTHGER MIN

C SECOND

INTEGEER SEC

C MIIJISECONDS

FRAL MSS

C FORUAT ID

INTEGER NFORM

C NUNTERR OF CHANNEIS

IITEGER NCHAN

C NUYBER OF SANPIES

INTEGER NSAMP

C SAMPIING RATE

REAL RATE

c

DNTEGER IP(40)

COYMON /HEADER/ ID, DNSTR, YEAR, DOFY, HOUR, MN, SEC, MSS, NFORY,

C

1 NGHAN, NSALP, RATE

C AT THIS TIIE, NOT ALL OF THE HEADER HAS INFORMATTON.

C THE FIRST 20 FRAMES HAVE BEFEN SET ASIDE FOR THE HBADER, C OF WHICH ONLY 14 FRAIES ARE CURRENTLY USED.

C

c

CALL, GEYTES(IDATA, I8, 0, 4, 0, 40)

$\mathrm{ID}=(\mathrm{IB}(1) \cdot 10+\mathrm{IB}(2)) * 10+\mathrm{IB}(3)$

$\mathrm{YGAR}=\mathrm{IB}(4) * 10+\operatorname{IB}(5)+1800$

$\mathrm{DOIY}=(\operatorname{IB}(6) \cdot 10+\operatorname{IB}(7)) * 10+\operatorname{IB}(\theta)$

HOUR $=\operatorname{IB}(0) \cdot 10+\operatorname{IB}(10)$

$\mathrm{MN}=\operatorname{IB}(11) * 10+\mathbb{I}(12)$ 


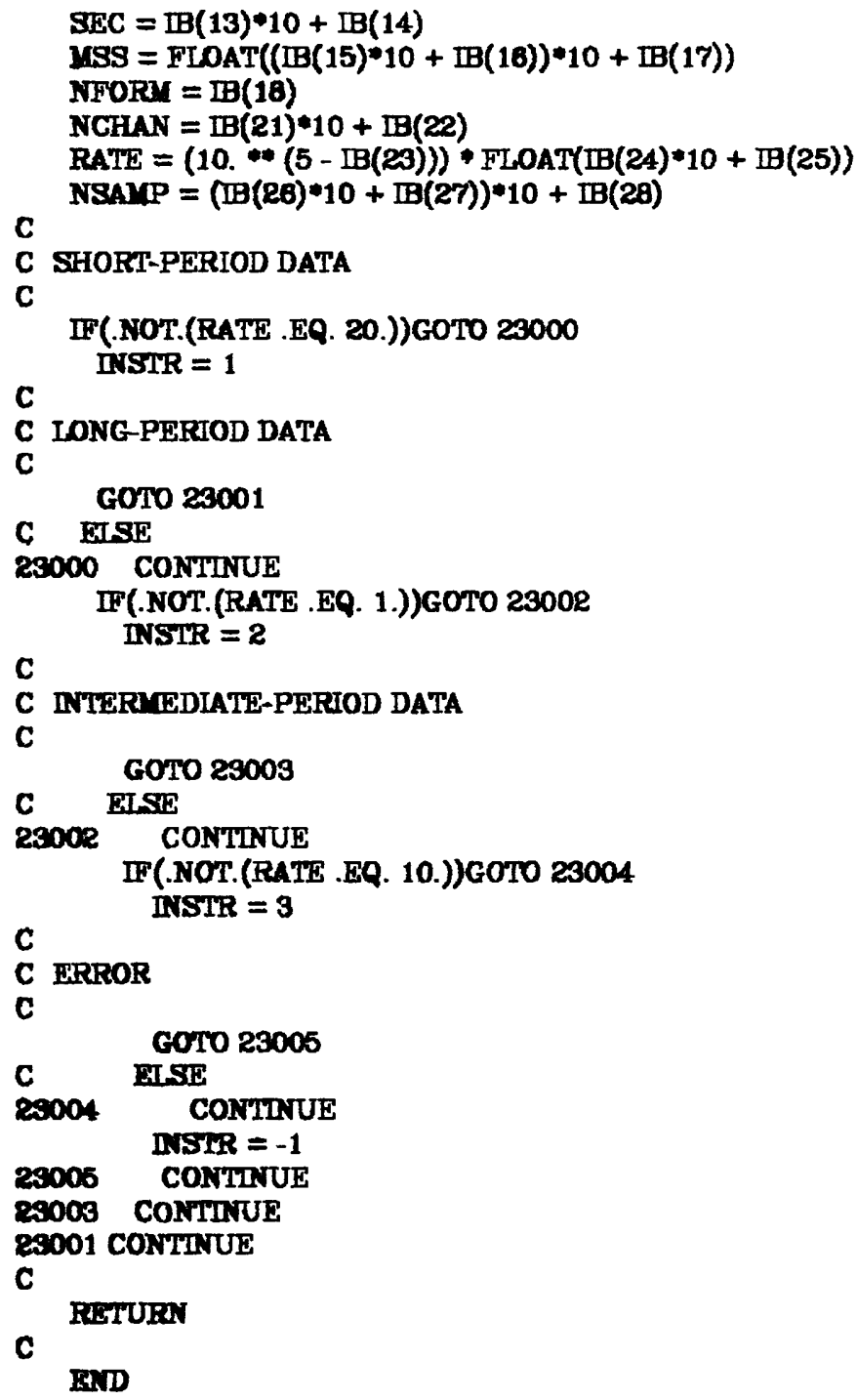


C

REAL FUNCTION DTPFMT(NFORY, MANT, CHAR)

C FUNCTION DTPFYT DECODES ONE DATA WORD FROM

C A NETWORK DAY TAPE, AND RETURNS IT AS

C THE FUNCTTON VALUE.

C IF NFORM $=1$, THE DATA IS IN SRO FORMAT:

C IF NFORM $=2$, THE DATA IS IN DWWSSN FORMAT.

C

C SRO AND ASRO DATA SAMPLES ARE RECORDED AS

C 16 BIT (2 FRAMES) WORDS USING A GAIN-RANGE

C FORMAT. THE FOUR MOST SIGNIFICANT BIIS OF

C FRANE 1 DEFINE THE GAIN FACTOR(GF) WHICH IS

C AN UNSIGNED INTEGER HAVING A VALUE FROM

C 0 TO 10. THE FOLLOWING BIT DEFINES THE STGN

C OF THE MANTTSSA, AND THE 3 REMAINING BITS

C PLUS THE 8 BITS OF FRAIE 2 CONTAIN SETSMTC

C DATA IN TWOS COMPLBMENT BINARY INTEGER FORM.

C ABSOLUTE AMPLITUDE IN DIGITAL COUNIS IS

C DERIVED AS FOLIOWS -

C

C $\triangle M P L T T U D E=$ MANTISSA * $2 *(10-\mathrm{GF})$

C

C THIS GIVES A DYNAMIC RANGE OF T2 DB, AN

C OPERATTNG RANGE OF 120 DB AND A MAXMUM

C AMPITIUDE OF +2,096, 128 TO -2,087, 152

C DIGTTAL COUNTS.

C

C DWWSSN DATA SAMPLES ARE RECORDED IN A

C 16-BIT TTOS COYPLEMTENT INTEGER FORUAT. THIS

C GIVES A DYNAIIC RANGE OF 98 DB AND

C A MAXINUM ANPLITUDE OF +38,707 TO

C -32,708 DIGTTAL COUNTS.

C

C DTPFYT ASSUMES THAT THE USERS MACHINE HAS

C A WOED LENGTH OF AT LEAST 16 BITS.

C

C PRANE IS DEFINED AS B CONTIGUOUS BYTE

C AIIGNED BITS BY THE TAPE DRTVE.

C

C

C CAIJS NO OTHER ROUTINE.

C

C PROGRAMIED BY MADELEINE ZIRBES

C BLPTEY IBER 15, 1890

C

C FORMAT ID - INPUT

INIEGER NFORM

C MANTTSSA - INPUT

INTEGER MANT

C CHARACTERTSTIC INPUT

DTIEGER CHAR

c

RREAL PWR(16)

DATA PWR/1024., 512., 256., 128., 4., 32., 16., 8., 4., 2., 1., .

C

15 , .25, .125, .0825, .03125/

C SRO FORMAT

C

IF(.NOT.(NFORM .EQ. 1))GOTO 23000

IT(. NOT.(MANT.GE.2040)) GOTO 23002

MANT = YANT -4006

23002

CONIINUE 


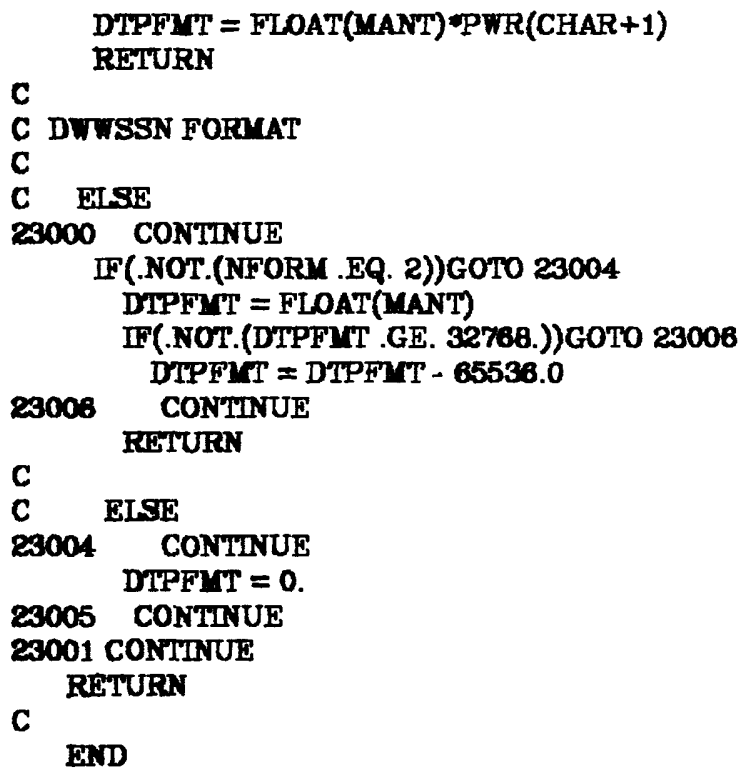


USERR CALLABBLE ROUTINES

IASNLU.FORTRAN

C

INTEGER FUNCTTON IASNLU (LUN, INDX)

C IASINLU ASSOCIATES A LOGICAL UNIT NUMBER

C WTTH AN INDEX TO THE HEAD AND DATA ARRAYS.

C CURREPTIY, THERE IS BUFFER SPACE TO BE

C ABLE TO READ 3 FIIFS STIVLTANEOUSLY.

C IASNLU RETURNS 1 IF AN INDEX WAS ASSIGNED,

C OR -1 IF ALL THE BUFFER SPACE HAS BEEN

C ALLOCATED.

C

C

C CAIIS:

C SAVE - MACHINE DEPENDENT

C PROGRAMTED BY MADEI EINE ZIRBES

C SEPTEEMER 15, 1980

C LOGTCAL UNIT NUMBER - INPUT

INTEGER LUN

C INDEX OF DATA BUFFER - OUTPUT

C INTTEGER INDX

C

C HEADER RECORD FIAG

DTTEGER HFLG(3)

C DATA RECORD FLAG

IITEGER DFLG( $(9)$

C NUMBER OF LOGICAL UNIT NUMRERS ASSIGNED DTTEGER NLUN

C LOCICAL UNIT NUNGERS ASSOCLATED WTTH DATA TNDEXES INTEGER LUUN(20)

C INDEX ASSOCTATED WTTH A LOGICAL UNIT NUMBER INTEGER ILUN(3)

C INDEXX OF MEXT DATA VALUE TO BE RETURRED

C IITIGGER NPTS(3)

C

COMDON /FLAGS/ HFLG, DFTG, NLUN, LLUN, IIUN, NPTS

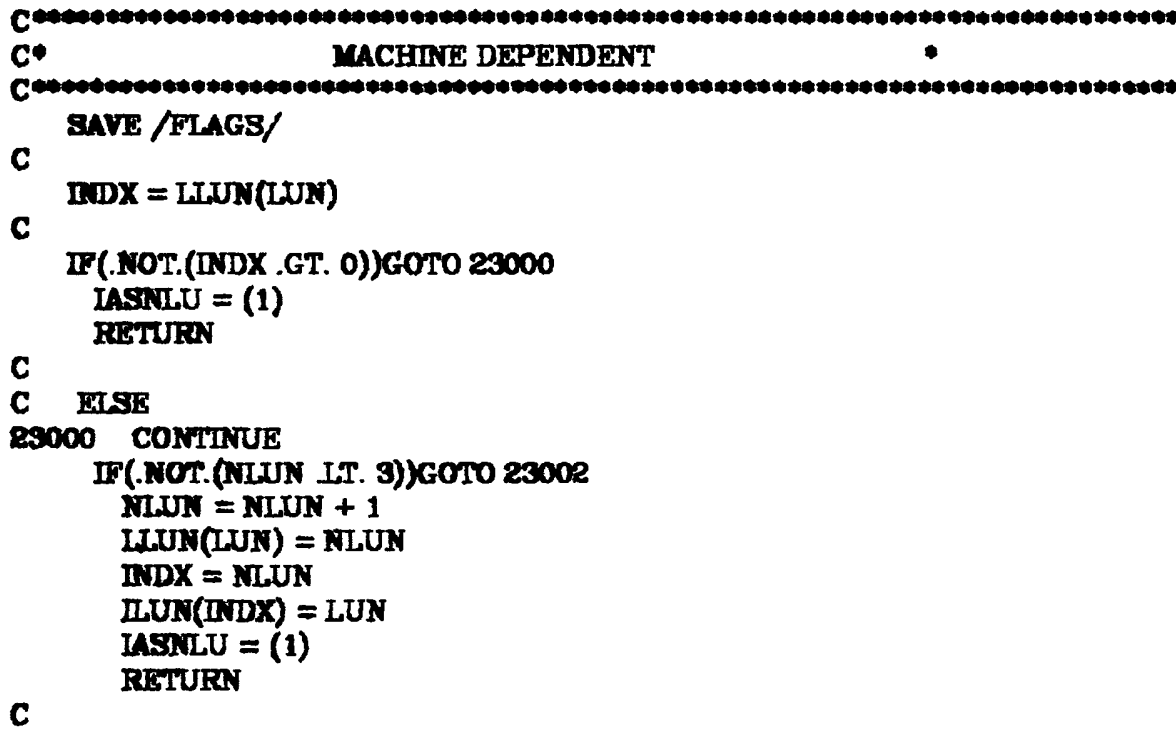


C HSE

23002 CONTINUE

IASNLU $=(-1)$

RETURN

23003 CONTINUE

23001 CONTINUE

C

END 
INTTFL.FORTRAN

C

SUBROUTTNE INITFL

C INITIALIZATTON OF FIAGS USED BY

C IRDHD AND IRDDAT IS DONE BY INITFL.

C INITFL YUST BE CAIIED BEFORE ANY

C REFFERENCE TO IRDHD OR IRDDAT TO

C INSURE READING THE HEADER AND

C DATA RECORD OF THE OUTPUT FIIES

C PRODUCED BY RETRV SUCCESSFULLY.

C

C

C CAISS NO OTHER ROUTINE.

C

C PROGRAMMED BY MADELLEINE ZIRBES

C SIEPTEMBER 15, 1980

C

C

C

C HEADER RECORD FLAG

INTEGER HFLG(3)

C DATA RECORD FIAG INTEGER DFLG(9)

C NUJBER OF LOGICAL UNTT NUMBERS ASSTGNED INTEGER NLUN

C LOGICAL UNIT NUYBERS ASSOCIATED WITH DATA INDEXES INTEGER LLUN(20)

C ASSOCLATES AN INDEX FITH A LOGICAL UNIT NUMBER ITMEGER ILUN(3)

C INDEX OF NEXT DATA VALUE TO BE RETURNED

C IITEGER NPIS(3)

C THE DATA READ FROM DISK IS SET TO ALLOW

C ACCESS TO THREE FILES SIMULTANEOUSLY

C

C NUMBER OF VALUES READ INTO DATA BUFFER BUFF RENL PTS(3)

C DATA BUFFER

C

REAL BUFF $(3,500)$

c

COMON /FLAGS/ HFLG, DFLG, NLUN, LLUN, ILUN, NPIS

C

COMMON /DATA/ PIS, BUFT

DATA HFLG, DFLG $/ 3 \circ 1,30 /$

DATA NPTS, PIS $/ 3 * 1,3 * 0.0 /$

C

DATA NLUN, ILUUN /0, 2000/

C

RETURN

ENDD 
RDDATA.FORIRAN

C

SUBROUTTNE RDDATA(LUN, INDX)

C RDDATA READS A DATA RECORD FROM

C LOGICAL UNIT NUMBER LUN.

C

C

C CAIIS NO OTHER ROUTINE.

C

C PROGRAMMED BY MADELEINE ZIRBES

C SEPTEMGER 15, 1980

C

C LOGICAL UNIT NUMBER - INPUT

INTEGER LUN

C ARRAY INDEX INTO WHICH THE DATA WILL BE READ - INPUT

C INTEGER INDX

C

NTIEGER I

C THE DATA READ FROY DISK IS SET TO ALLOW

C ACCESS TO THREE FIIES SIMULTANEOUSLY

C

C NUMBER OF VALUES READ INTO DATA BUFFER BUFF REAI PTS(3)

C DATA BUFFER

C

RTEAL BUFF(500, 3)

C

COMMON /DATA/ PTS, BUFF

READ(LUN) PIS(INDX), (BUFF(I,INDX), I=1,PIS(INDX))

C

C

RETURN

IEND 
RDHEAD.FORIRAN

C

SUBROUTINE RDHEAD(LUN, INDX, TF)

C RDHEAD READS A HEADER RECORD FROM

C LOGICAI UNIT NUMAER LUN. TF $=1$

C IF THIS IS THE IAST RECORD OF

C THE FIIE.

C

C

C CALIS NO OTHER ROUTINE.

C

C PROGRAMMED BY MADELETNE ZIRBES

C STIPTEM MERR 15, 1880

c

C LOGICAI UNIT NUMBER - INPUT

INTEGER LUN

C ARRAY INDEX INTO THE DATA WILC BE READ - INPUT INTEGER INDX

C BAD OF DATA FLAG - OUTPUT

C

INTEGER TF

C

ITIEGER I

C THE DATA READ FROM DISK IS SET TO ALLOW

C ACCESS TO THREE FIIES STULTANEOUSLY

C

C STATTON CODE - CHARACTER STRING

INTEGER CODE $(4,3)$

C INSTRUJEFT CODE - CHARACTER STRRIG

INTEGER CHN(4,3)

C STATTON LATITUDE

BNEAL IATY(3)

C STATION LONGITUDE

FWNL. ION(3)

C STATION RLEVATION

RDALL ETEV(3)

C STMRT TINE OF DATA

C YWAR

INTEGER SYEAR(3)

C DAY OF YEAR

ITISGER SDOFY(3)

C HOUR

INIEGPR SHOUR(3)

C MTUIE

IVISGER SIINV(3)

C sixconds

RARAL SSECS(3)

C SAMPINIG RATE

REAL RATE(3)

C NORMMLTLATON CONSTANT

Fon AO(3)

C NUNGER OF POLES

INTEGER NP(3)

C POLES

COMIPIDX POLES(20,3)

C NUNBER OF ZREOES

IVIECSR NZ(3)

C ZATPOES

C

COMPLEX ZEROES $(10,3)$

COMON /HEAD/ CODE, CHN, IAT, LON, ELEV, SYEAR, SDOFY, SHOUR,

C 1 sITN, SSECS, RATE, AO, NP, POLES, NZ, ZEROES 
$T F=0$

READ(LUN) (CODE(I,INDX), I=1,4), (CHN(I,INDX), I=1,4), IAT(INDX),

1 ION(INDX), EIEV(INDX), SYEAR(INDX), SDOFY(INDX), SHOUR(INDX),

c

1 SIMIN(INDX), SSECS(INDX), RATE(INDX), AO(INDX), NP(INDX), (POLES(

C IF TF EQUALS ONE,

C THIS IS A NULL HEADER RECORD -

C IAST RECORD IN THE FIIE

C

IF(.NOT.(AO(INDX) .EQ. 0.0))GOTO 23000

C

$$
\text { TF }=1
$$

23000 CONTINUE

RETURN

C

END 
C

INIEGER FUNCTION IRDDAT (INDX, VALU)

C FUNCTION IRDDAT SEQUENTIALIY READS

$C$ THE NEXT DATA POINT OF A DATA RECORD

C ON EACH CALL, RETURNING IT IN VALU.

C IRDDAT RETURNS 1 ON A SUCCESSFUL.

C READ, O FOR END OF DATA, OR END OF

C FIIE, AND - 1 FOR AN ERRROR.

C

C

C CAIJS:

C RDDATA

C SAVE - MACHINE DEPENDENT

C

C PROGRAMNED BY MADETEINE ZIRBES

C SEPTEN IBER 15, 1980

C

C APRAY INDEX - INPUT

INIEGER INDX

C DATA VALUE - OUTPUT

REAL VALU

C

C LOGICAL UNIT NUMBER INTEGER LUN

INTEGER I

C

C THE DATA READ FROY DISK IS SET TO ALJOW

C ACCESS TO THREE FILES SIMULTANEOUSLY

C

C NUNGER OF VALUES READ INTO DATA BUFFER BUFF REAI PTS(3)

C DATA BUFFER

C RJAL BUFF(500, 3)

C HEADER RECORD FLAG INIEGER HFLG(3)

C DATA RECORD FLAG IIIITGER DFIG(3)

C NUYIERR OF IOGICAL UNIT NUYBERS ASSIGNED IIIIZGER NLUN

C LOGICAL UNTT NUMBERS ASSOCIATED WTHH DATA INDEXES INIEGER LLUN(20)

C IIDEX ASSOCLATED WTTH A LOGICAL UNTT NUMBER INTEGER IIUN(3)

C INDEX OF NEXT DATA VALUE TO BE RGTURNED

C INTEGER NPTS(3)

C COMMON /DATA/ PIS, BUFF

COMMON /FLAGS/ FFLG, DFLG, NLUN, LUUN, IUUN, NPTS

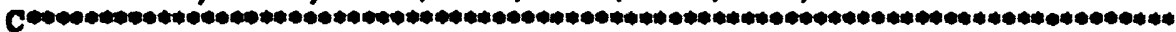
c* MACHDE DEPENDENT

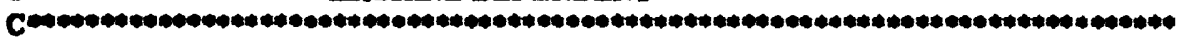
SAVE /DATA/, / FAGS/

C

VALU $=0.0$

C

LNN = IUN(WDX)

C

C READ IN NEXT DATA RECORD

C

IF(.NOT.(FLOAT(NPTS(TNDX)) .GT. PTS(INDX))) GOTO 23000 


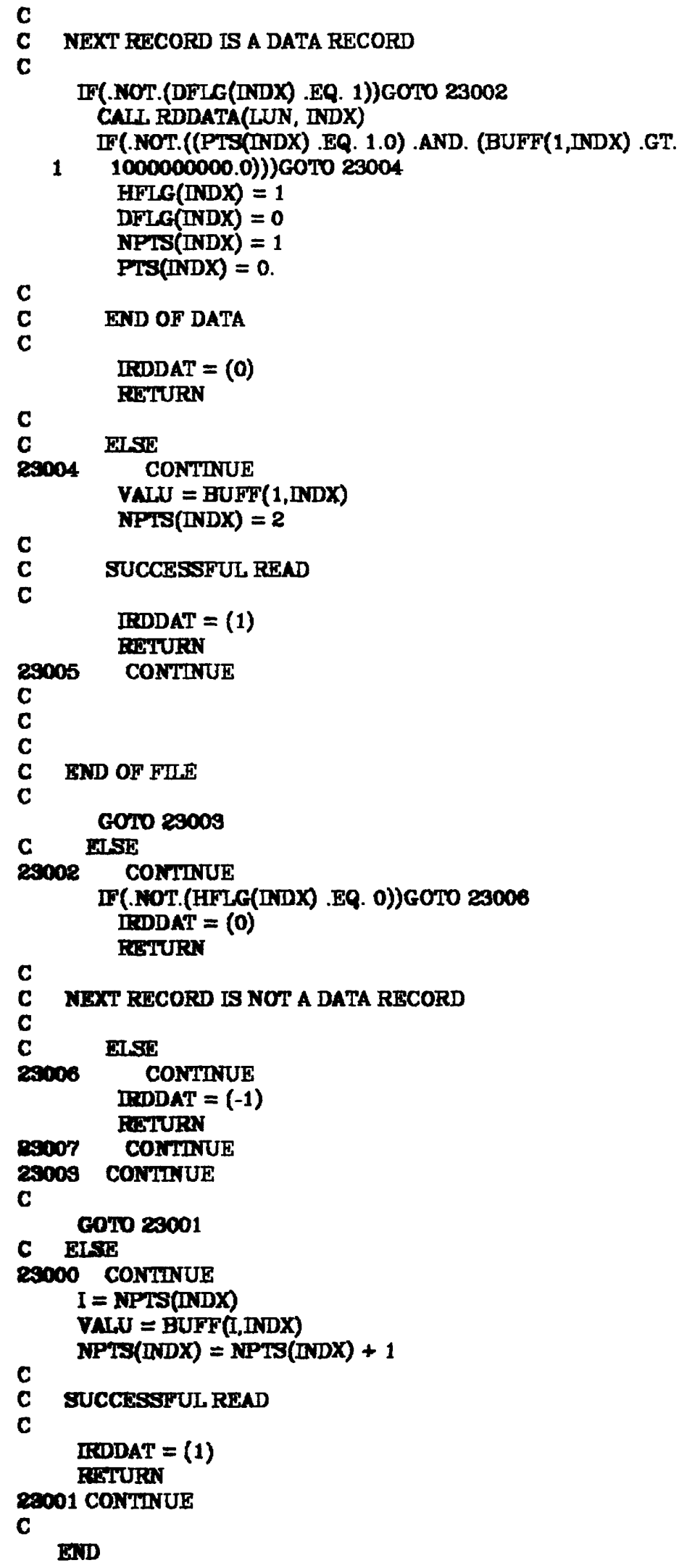


C

INIEGER FUNCTION IRDHD (INDX)

C FUNCTION IRDHD READS THE NEXT HEADER

C RECORD FROM LOGICAL UNIT NUMBER LUN.

C IT WILL ALWAYS TRY TO RETURN THE

C NEXT HEADER RECORD, READING OVER

C INTERVEDIATE DATA RECORDS IF

C NECESSARY. IT RETURNS 1 ON A

C SUCCESSFUL READ, 0 ON END OF FIIE.

c

C

C CALIS:

C IRDDAT

C RDHEAD

C SAVE - MACHINE DEPENDENT

C

C PROGRAMTED BY MADELFTNE ZIRBES

C SEPTEMBER 15, 1860

C

C ARRAY INDEX - INPUT

INTEGER INDX

C

C LOGICAL UNTT NUMBER INTEGER LUN

C

C THE DATA READ FROY DISK IS SET TO ALLOW

C ACCESS TO THREE FIIES SIVULTANEOUSLY

C

C STATTON CODE - CHARACTER STRING INTEGER CODE $(4,9)$

C INSTRUMENT CODE - CHARACTER SITRING INIEGER CHN $(4,3)$

C STATION IATTTUDE REAL IATY(S)

C STATION LONGITUDE RENL LON(3)

C BTATION EILVVATION ERAI, ELEV(s)

C START TI IE OF DATA

C YEAR

INTEGER SYEAR(3)

C DAY OF YEAR INIEGER SDOFY(9)

C HOUR INTEGER SHOUR(3)

C YIINUTE INIEGER SITN(3)

C BECONDS REAL SSECS(3)

C SAMPLING RATE IREAL RATE(B)

C NORTALIZATION CONSTANT RGAL AO(3)

C NUNGEER OF POLES INTEGER NP(3)

C POLES COMPLEX POLES $(20,3)$

C NUNABER OF REROES INTEGER NZ(3)

C ZEROES

C CONPLEX ZEROES(10,3) 


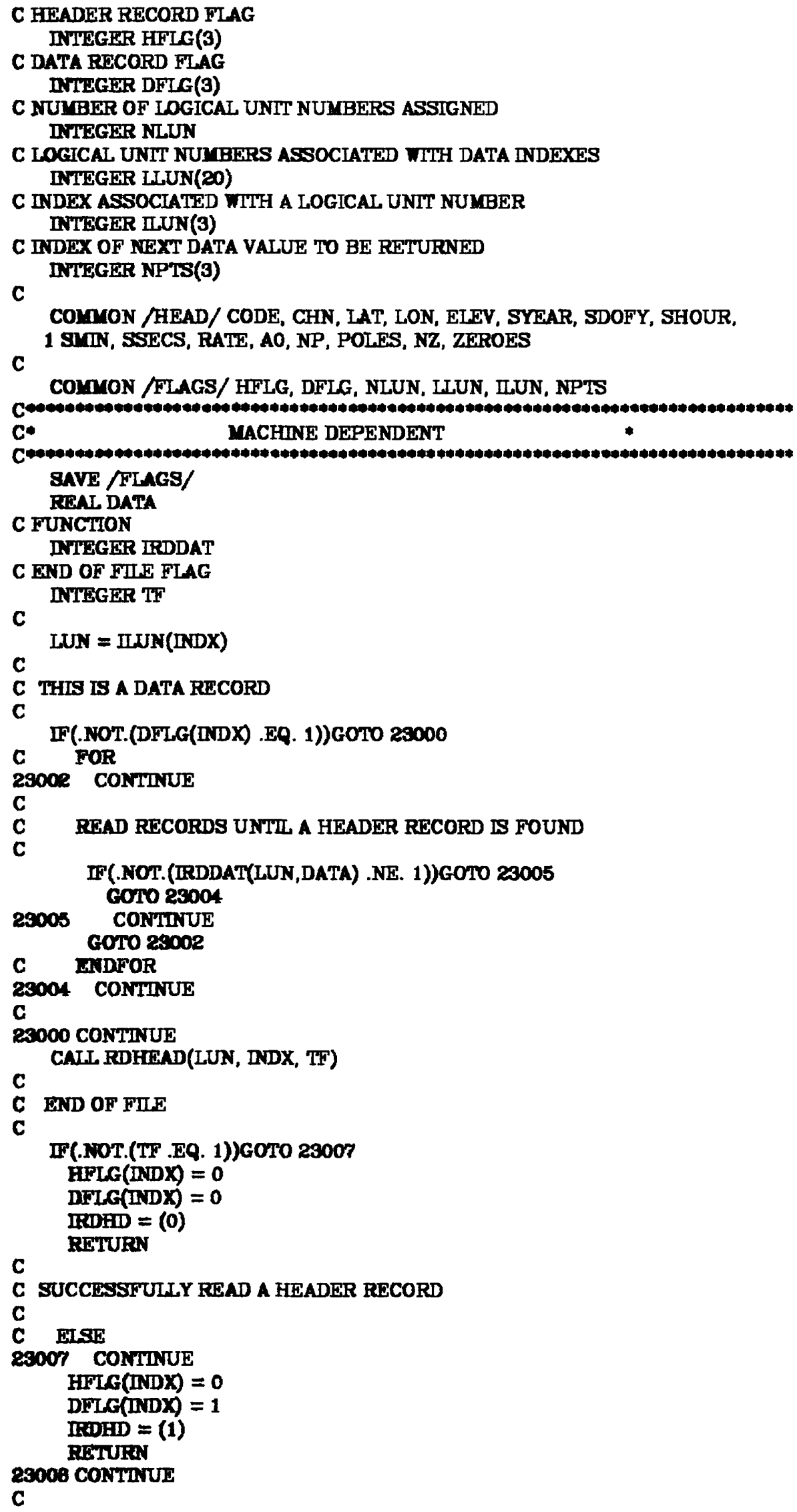


END 
RESP.FORTRAN

c

COMPLEX FUNCTION RESP(INDX,OMEGA,IFL)

C RESP RETURNS T(S) $=A 0 *(S-Z(1)) * \ldots *(S-Z(M)) /(S-P(1)) * \ldots *(S-P(N))$,

$C$ WHERE $P, Z$, AND S ARE COMPLEX. $S=C M P L X(0,0 M E G A)$.

C IFL FLAGS THE CALILERS DESTRE FOR

C DISPIACEVT (IFL=1), VELOCITY (IFL=2),

C OR ACCETLERATION (IFL=3).

C

C

C CALIS NO OTHER ROUTINE.

C

C PROGRAMMED BY R. BUIAND

C AUGUST 23, 1979

C

C ARRAY INDEX - INPUT

INTEGER INDX

C ANGULAR FREQUENCY - INPUT

REAL OMEGA

C FLAG - DETERMINES OUTPUT UNITS - INPUT

C INTEGER IFL.

\section{COMPLEX $S$ \\ COMPLEX D \\ INTEGER J}

c

C THE DATA READ FROY DISK IS SET TO AJLOW

C ACCESS TO THREE FIIES SIVULTANEOUSLY

C

C STATTON CODE - CHARACTER STRING

INTEGER CODE $(4,3)$

C INSTRUMENT CODE - CHARACTYR STRING INTEGER CHM $(4,3)$

C STATTON LATTTUDE

FEAL I ATT(3)

C STATION LONGITUDE

REPAL LON(3)

C STATTON ELEVATTON

REALL EIEV( $(9)$

C START TIIE OF DATA

C YBAR

IIYEGER SYEAR(3)

C DAY OF YEAR

INTEGER SDOFY(3)

C HOUR

IFTEGER SHOUR(3)

C MIIUTTE

IYIEGER SUIN(3)

C SECONDS

REAL SSECS(3)

C SAYPLDNG RATE

RRSAL RATE(3)

C NORMALIZATYON CONSTANT

REAL AO(3)

C NUYABR OF POIES

INTEGER NP(3)

C POLES

COMPLEX POLES $(20,3)$

C INUMERR OF ZEROES

INTEGER NZ(3)

C ZITROES

C

CONPLEX ZEROES $(10,9)$ 


\section{APPENDIX C}

\section{GBYTES}

Gbytes is one of the routines used by the commands retrv and ndtsum that is machine dependent.

We have obtained examples of gbytes for several machines. The documentation preceeding the code for each example lists the machines on which the code can be run.

We would like to thank Dr. Steve Pazan at Scripps Institution of Oceanography for his help in supplying some of the examples. 
COYMON /HEAD/ CODE, CHN, LAT, LON, ELEV, SYEAR, SDOFY, SHOUR,

C

1 SUMN, SSECS, RATE, A0, NP, POLES, NZ, ZEROES

IF(.NOT.(NP(INDX).LE.0))GOTO 23000

RESP $=(0.0)$

RETURN

23000 CONTINUE

$\mathrm{D}=(1,0$.

C

$\mathbf{S}=$ CMPIX $(0 .$, OMEGA)

c COMPUTE THE CONTRIBUTHON OF THE ZEROES IF ANY

C

DO $23002 \mathrm{~J}=1, \mathrm{NZ}$ (INDX)

$D=D *(S-Z E R R O E S(J, I N D X))$

2300R CONITINUE

C

C CONPUTE THE CONTRIBUTION OF THE POLES

C

DO $23004 \mathrm{~J}=1, \mathrm{NP}$ (INDX)

$\mathrm{D}=\mathrm{D} /(\mathrm{S}-\mathrm{POLES}(\mathrm{J}, \mathrm{INDX}))$

28004 CONTINUE

C

C

C DEBPLACEMENT

C

IF(.NOT.(IFL .EQ. 1))GOTO 23006

RESP $=(A O(I N D X) \cdot D)$

C RETURN

C VRLOCITY

C EISE

20000 CONTTNUE

IF(.NOT.(IFL .EQ. 2))GOTO 29008 RESP $=(A O(I N D X) D / S)$

C

C ACCELERATION

C

C EISE

25008 CONTINUE

IF(.NOT.(IFL .EQ. 3))GOTO 23010

RASP $=\left(A O(\operatorname{INDX}) \cdot D /\left(S^{*} \cdot S\right)\right)$

28010 CONTINUE

25000 CONITIUE

2S007 CONTINUE

END 
IBM 360 VERSION

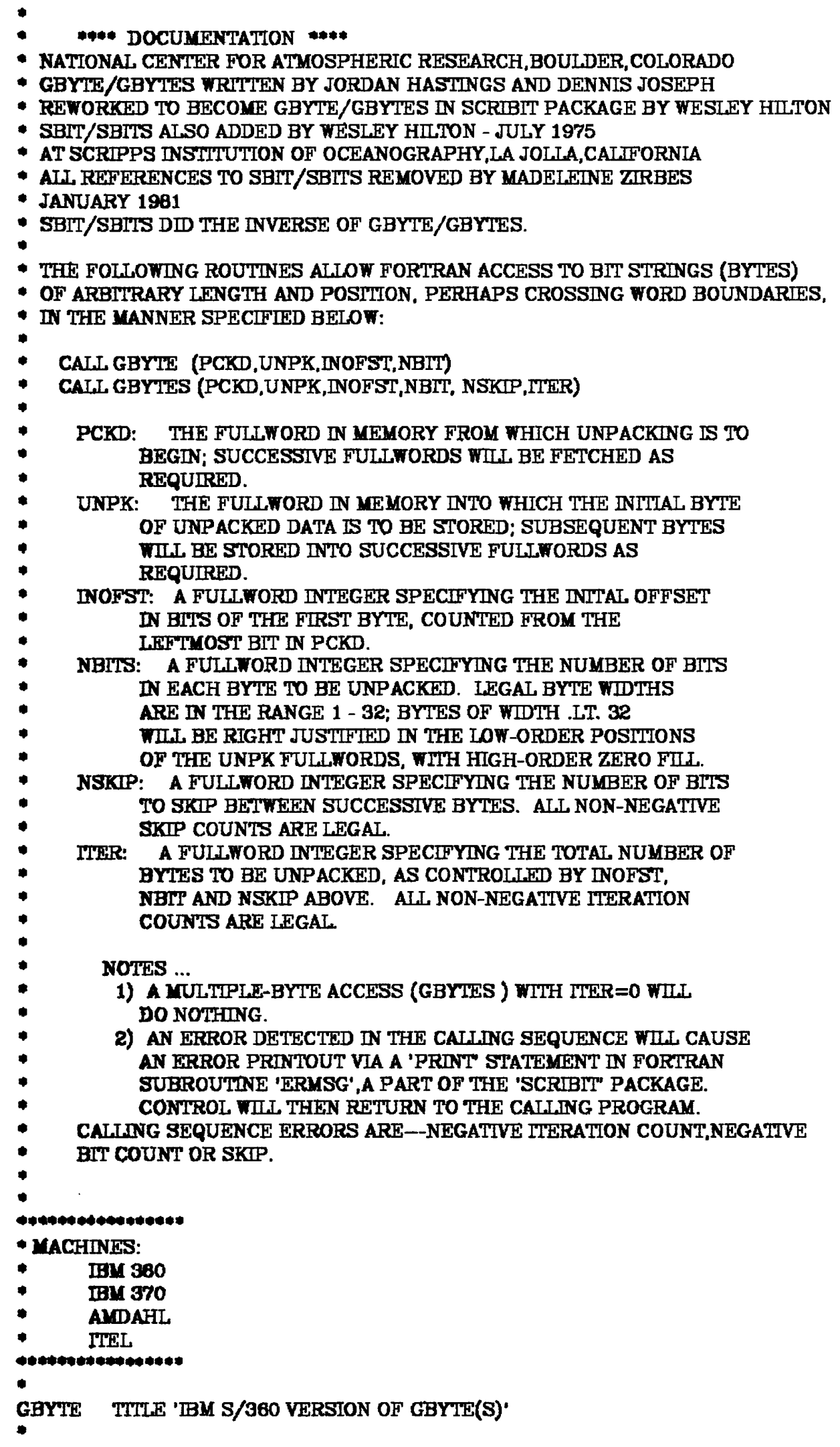


PCKD

UNPK

OFST

NBIT

NSKPR

\section{-}

NSKP

IIER

BITR

BITS

WORK

HIWD

LOWD

BASE

$\rightarrow$

$-$

BYTE

NBFW

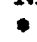

$+$

GBYTES

-

GBYTE DS

SAVE $\quad(14,12)$

$\bullet$

INIT

BALR

USING

I.M

I.

I.

SR

CR

BE

BI.

AR

IF

SR

BM

SIH

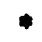

$\bullet$

AR

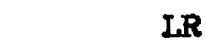

IR

- TOP OF ITERATION

TOP
GBYTE, 15

X'o'

$X ' 1$

$\times 2$

$\mathrm{X} \cdot \mathbf{3}^{\prime}$

$X 4^{\prime}$

$X^{\prime} 4^{\prime}$

X'5

$X^{\prime} \mathbf{B}^{\prime}$

$X ' 7$

X'B

$X^{\prime} 9^{\prime}$

$X^{\prime} A^{\prime}$

$X^{\prime} B^{\prime}$

$\mathrm{X}^{\prime} \mathrm{C}^{\prime}$

$X ' E^{\prime}$

$X^{\prime} D^{\prime}$

$\mathbf{X}^{\prime} \mathrm{F}^{\prime}$

4

CODE

ERMSG

10(15)

X'5'

CI5'BRMSG'

PRTIE ENTRY POINT

GBYTE

GBYTES, 15

$(14,12)$

NSKP,ITER, 4*NBFW(1)

NSKP, O(NSKP)

ITER, O(,ITER)

INIT

IIER, $=\mathrm{H}^{\prime} 1^{\prime}$

BAST, 0

-BASE

PCKD,NBIT, O(1)

OFST,O(OFSI)

NBIT, O(, NBIT)

ZEFO, ZERO

IIER, ZERO

EXXTT

FAII

NSKP,NBIT

WORK, $=H^{\prime} 32$

TORK,NBIT

FAII

WORK,RJUST+2

NBIT,NSKP

BIIS,OFST

NORK $_{2}=\mathrm{H}^{\prime} 64^{\prime}$

OFST,BITS

OFST,5
CONSTANT ZERO

ADDR OF PACKED ARY ELEMENT

ADDR OF UNPACKED ARY ELEMENT

INITIAL BIT OFFSET

BYTE IENGTH IN BITS (.IE.32)

SAME REG USED FOR (NSKP+2*NBIT) IF

ITERATTVE BIT SKIP

IIEERATION COUNT (.GE.0)

BITS REMAINING IN HIWD

BIIS TO SHIFT (OFST OR NSKP)

WORK REGISTER

HI-ORDER WORD (EVEN REGISTER)

LO-ORDER WORD (ODD REGISTER)

BASE ADDR REGISTER

RETURN ADDR (NOT MODIFIED)

SAVE AREA ADDR (NOT MODIFIED)

RESULTANT BYTE

NUMBER OF BYTES/FULL WORD

10

ALTERNATE ENTRY POINT

ENTRY PT ADR ESTB IN R15 BY CALCER

SAVE ALI REGS EXCEPT 13

PICK UP NSKIP, ITER PARM ADDRESSES

PICK UP BIT SKIP COUNT

PICK UP ITERATION COUNT

JUMP INTO CODE PROPER

FORCE HALFWD ALIIGNMENT

ALT ENTRY ADDR ESTB IN R15 BY CALCER

SAVE ALL REGS EXCEPT 13

MAKE ITERATION COUNT ONE

RE-ESTABLISH ADDRESSABIITYY

PICK UP ARY AND PARAMETER ADDRESSES

PICK UP INITIAL OFFSET BIT COUNT

PICK UP NO. OF BITS/BYTE TO UNPACK

ZERO OUT REG 0

TEST ITERATION COUNT

FETURN,DO NOTHING,IF ITER COUNT ZERO

FAII. ON NEGATTVE TIERATION COUNT

ADD NBIT TO NSKP FOR MULT ACCESS

MAKE WORK $=32$

COMPIENENT NBTIS (MODULO 32)

IF NOT .LE.32, FAII

EISE USE TO SET UP BYTE SHIFT COUNT

OF SRL (RX) INSTR IN STORE SEQ

REG NOW BECOMES NSKP2=

(NSKP+2*NBIT) FOR LOOP TEST

MAKE BITS TO SHIFT = INITIAL OFFSET

MAKE WORK $=64$

COPY BITS REMAINING TO SHIFT TO OFST DIVIDE BY 32 TO GET WORDS TO SKIP 


\section{CDC VERSION}

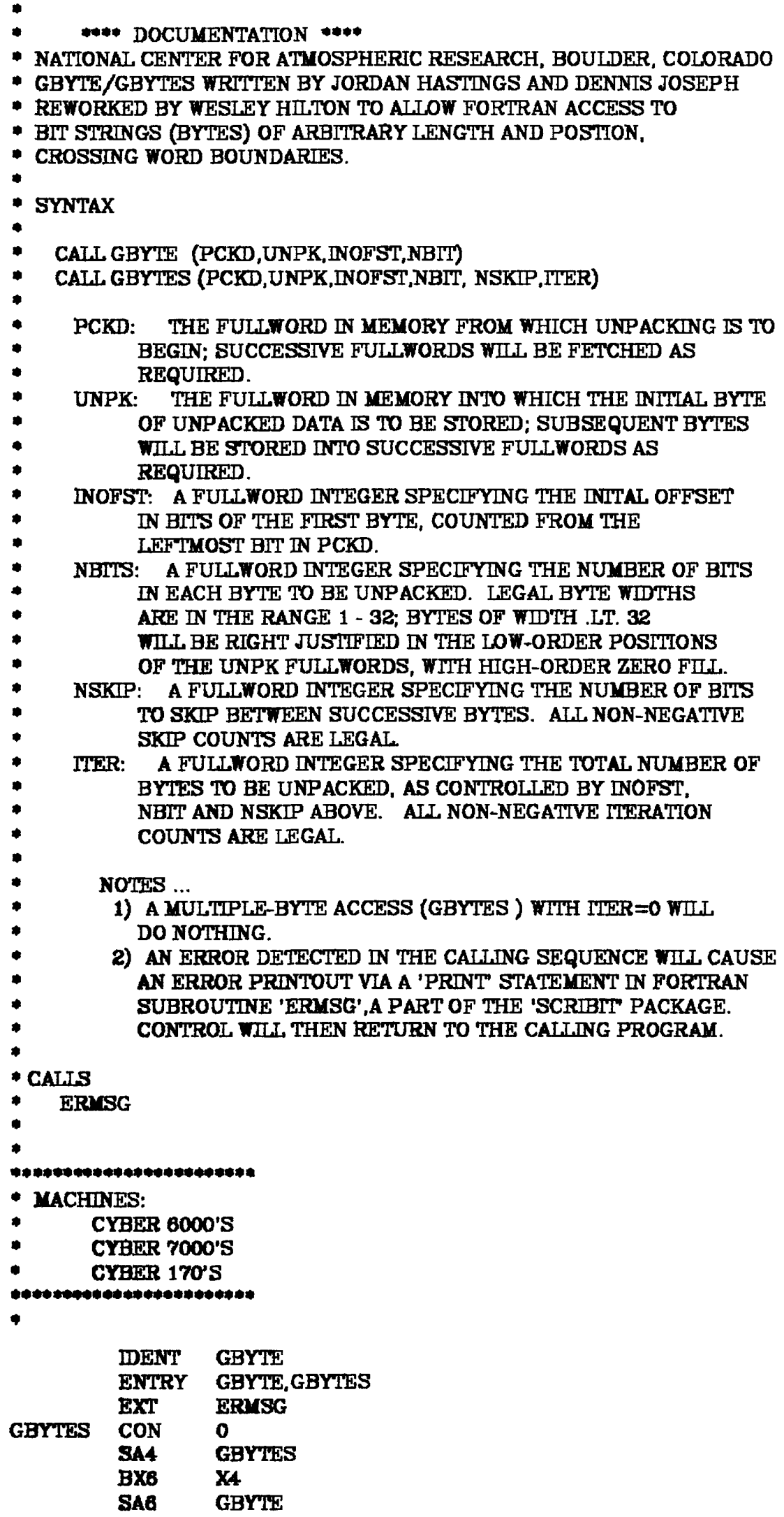




$\begin{array}{ll}\text { SLA } & \text { OFST,2 } \\ \text { AR } & \text { PCKD,OFST } \\ \text { L } & \text { HIWD,O(,PCKD) } \\ \text { SLA } & \text { OFST,3 } \\ \text { SR } & \text { BITS,OFST } \\ \text { I } & \text { LOWD,NBFW(,PCKD) } \\ \text { LR } & \text { BITR,WORK }\end{array}$

- NOW LEFT JUSTIFY DESIRED SUBSTRING IN HIWD

\begin{tabular}{|c|c|c|c|}
\hline SHIF"T & $\begin{array}{l}\text { STH } \\
\text { SLDL }\end{array}$ & $\begin{array}{l}\text { BIT'S, }+6 \\
\text { HIWD, } \cdots\end{array}$ & $\begin{array}{l}\text { SET UP BITS AS SIDL SHIFT COUNT } \\
\text { SHIFT IEFT DOUBLE IOGICAL, HIWD/LOWD }\end{array}$ \\
\hline - RIGHT & JSTTFY DE & RED SUBSTRING IN CAL & ING PROGRAM UNPACKED ARRAY \\
\hline & LR & BYTE,HIWD & COPY HIWD TO BYTE \\
\hline RJUST & SRL & BYTE, $\cdots$ & SHIFT RIGHT(WITH HI-ORDER ZERO FILI) \\
\hline - BY SH] & r COUNT & T UP AT INITTALIZATION & EQUENCE \\
\hline & ST & BYTE,O(UNPK) & FILL BYIE INTO UNPACKED ARRAY \\
\hline & BCT & ITER,MORE & DECR ITER COUNT, CONTINUE THLL ZERO \\
\hline EXIT & MVI & $12(13), X^{\prime} F F^{\prime}$ & FLAG RETURN LINKAGE \\
\hline & RETURN & $(0,12)$ & RESTORE CALIJNG PROG REGS (MACRO) \\
\hline MORE & SR & BITR,BITS & DECR BITS REMAINING BY BITS SHIFTED \\
\hline & IA & UNPK,NBFW(UNPK) & AND INCR UNPACKED ARY POINTER \\
\hline & IR & BITS,NSKP2 & GET (NSKP+2*NBITS) \\
\hline & SR & BITS,BITR & SUBTRACT BITS REMAINING \\
\hline & $\mathbf{C R}$ & BISS,ZERO & COMPARE TO ZERO \\
\hline$*$ & $\mathbf{L R}$ & BITS,NSKP & $\begin{array}{l}\text { IN ANY CASE,SET BITS TO SKIP TO } \\
\text { (NSKP+NBIT) }\end{array}$ \\
\hline & BNH & SHIFT & DATA IS ALREADY IN REGS,NO FETCH \\
\hline & SR & BITS,BITR & SUBTRACT CURRENT REMAINING BITS \\
\hline & AR & BIIS, WORK & ADD 64 TO RESET TO PCKD POINTER \\
\hline & $\mathbf{B}$ & TOP & GO FETCH MORE DATA FROM MFMORY \\
\hline & & & \\
\hline FAII & ST & UNPK, GBARR & PUT UNPACKED ARRAY ADR AS ARG \\
\hline & $\mathbf{S T}$ & PCKD,GBERR+2^NBFW & POINT TO PACKED ARRAY AS ARG \\
\hline & $\mathbf{S T}$ & 14, GBRTN & RETURN ADDRESS IS ARG \\
\hline & IA & 1,GBERR & POINT TO ARG LIST WTTH REG ONE \\
\hline & LR & 2,13 & SAVE CALIING PROGRAM SAVE AREA PTR \\
\hline & IA & 13,GAREA & POINT TO LOCAL SAVE AREA \\
\hline & $\mathbf{S T}$ & $13,8(0,2)$ & IEFT-RIGHT IINK LOCAL SAVE AREA \\
\hline & ST & $2,4(0,13)$ & INTO CHAIN OF SAVE AREAS \\
\hline & L & $15,=A($ ERMSG $)$ & SET UP BRANCH \\
\hline & BAIRR & 14,15 & GO PRINT ERROR MESSAGE \\
\hline & L & 13,GAREA+4 & RETOAD CALLING SAVE AREA ADR \\
\hline & I & $14,12(13)$ & RECOVER RETURN ADDRESS \\
\hline & B & EXIT & \\
\hline GAREA & DS & $18 \mathrm{~F}$ & \\
\hline GSAVE & $\mathrm{DS}$ & $2 F$ & \\
\hline GBRTN & $\mathrm{DS}$ & 1F & \\
\hline GMSG & DC & $F^{\prime \prime} 11^{\prime}$ & SELECT GBYTE DIAGNOSITC MESSAGE \\
\hline GBARR & DS & $1 F$ & \\
\hline GBERR & DC & A(GBRTN) & POINT TO RETURN ADDRESS \\
\hline & DC & A(GMSG) & POINT TO MESSAGE SELECT \\
\hline & DS & & WILL POINT TO PACKED DATA \\
\hline & DC & A(GBARR) & POINIS TO UNPACKED ARRAY ADDRESS \\
\hline
\end{tabular}

CONVERT TO BYTES

INCR PACKED ARRAY POINTER

AND LOAD NEW HIWD

MULTIPY BYTES BY 8 TO GET BITS

AND DECR BITS REMAINING TO SHIFT

PICK UP NEXT PACKED WORD

RESET BITR TO 64

SET UP BITS AS SLDL SHIFT COUNT

SHIFT LEFT DOUBLE LOGICAL, HIWD/LOWD

COPY HIWD TO BYTE

SHIFT RIGHT(WITH HI-ORDER ZERO FILL)

FILL BYTE INTO UNPACKED ARRAY

DECR ITER COUNT, CONTINUE THLL ZERO

FLAG RETURN IINKAGE

DECR BITS REMAINING BY BITS SHIF"TED

AND INCR UNPACKED ARY POINTER

(NSKP+2"NBITS)

COMPARE TO ZERO

IN ANY CASE,SET BITS TO SKIP TO

(NSKP+NBIT)

SUDTRACT CURRENT REMANING BIS

ADD 64 TO RESET TO PCKD POINTER

GO FETCH MORE DATA FROM MFMORY

PUT UNPACKED ARRAY ADR AS ARG

OIT TO PACKED ARRAY AS ARC

POINT TO ARG IIST WTTH REG ONE

POINT TO LOCAL SAVE AREA

IEEFT-RIGHT IINK LOCAL SAVE AREA

SET UP BRANCH

GO PRINT ERROR MESSAGE

REIOAD CALI.ING SAVE AREA ADR

RECOVER RETURN ADDRESS

POINTS TO UNPACKED ARRAY ADDRESS 


\begin{tabular}{|c|c|c|c|}
\hline & BX1 & $\mathbf{x} 0 \times \mathbf{X} 1$ & .GET THE BYTE ISOLATED IN X1 \\
\hline & PL & B1,RTJST & .IF WE ARE DONE,JUMP \\
\hline & $\mathbf{M X 0}$ & 1 & .IF \\
\hline & SB3 & $-\mathbf{B 1}$ & - \\
\hline & SB3 & B3-1 & . \\
\hline & AXO & $\mathrm{B3}, \mathrm{XO}$ & - \\
\hline & BX2 & $\mathrm{X}^{*} \times \mathbf{X 0}$ & .ISOLATE 2ND PART OF BYTE \\
\hline & BX1 & $-\mathrm{XO}+\mathrm{X} 1$ & .CHOP OFF TRASH IN 1ST PART OF BYTE \\
\hline & BX1 & $\mathbf{X} 1+\mathbf{X} 2$ & .COMBINE PORTMONS \\
\hline RTIST & $\mathbf{Z R}$ & X4,LETT & .IF SIGN BIT IS INVOLVED,JUMP \\
\hline & $\mathbf{A X 6}$ & $\mathbf{B 1}, \mathbf{X 1}$ & .RTGHT JUSTTFY THE BYTE \\
\hline STR & SAB & B7 & .STORE IT IN OUTPUT FIELD \\
\hline & ZRR & BA,GBYTE & .IF DONE,LEAVE \\
\hline & SB6 & B6-1 & .IF NOT DONE COUNT \\
\hline & SB7 & $B 7+1$ & \\
\hline & IX4 & $X 4+X 5$ & \\
\hline & $\mathbf{S X 4}$ & $\mathrm{X} 4+\mathrm{B} 5$ & .COFFSET = OLD OFFSET+ BYTE SIZE + SKIP SIZE \\
\hline & JP & LOOP & GO LOOP \\
\hline LEFFT & SB1 & $\mathbf{X} 5$ & .GET BYTE SIZE \\
\hline & IXX & B1,X1 & .RIGHT JUSTIFY THE BYTE \\
\hline & JP & STR & .GO STORE IT \\
\hline - SET UP & ARGUME & IS FOR ERR & TNTOUT \\
\hline & IFEQ & $F, 1$ & ASSEMBLE IF MNF/RUN CALLED \\
\hline GBERR & SB3 & B1 & STRING POINTER \\
\hline & SX8 & B2 & PUT ARRAY ADDRESS IN MEMORY \\
\hline & SAB & $\mathrm{ADR}$ & AT ADR \\
\hline & SB4 & ADR & AND PODNT AT IT \\
\hline & SB1 & GBYTE & RETURN ADDRESS \\
\hline & SB2 & $=11$ & MESSAGE SELECT \\
\hline & $\mathrm{RJ}$ & ERMSG & GO PRINT OUT MESSAGE \\
\hline & $\begin{array}{l}\text { JP } \\
\text { ENDIF }\end{array}$ & GBYTE & SHOULD NOT BE HERE \\
\hline & IFEQ & $* \mathbf{F}, \mathbf{Z}$ & ASSEMBIE IF FTN CAICTED \\
\hline ARGLST & CON & GBYTE & ARG 1 IS RETURN ADDRESS \\
\hline & CON & $=11$ & .ARG 2 IS MESSAGE SELECT \\
\hline & CON & 0 & ARG 3 WIL BE STRING \\
\hline & CON & ADR & ARG 4 VIJ BE ARRAY ADDRESS \\
\hline GBERR & SA1 & ARGLST & POINT TO ARG LIST FWN STYLE \\
\hline & 8X8 & B1 & GET STRING POINTER \\
\hline & SAB & ARGIST+2 & MAKE STRING ARG 3 \\
\hline & $\mathbf{5 \times 6}$ & & GET ARRAY ADDRESS \\
\hline & SAB & ADR & MAKE ARG 4 ARRAY ADDRESS \\
\hline & RJ & ERMSG & GO PRINT OUT MESSAGE \\
\hline & JP & GBYTE & SHOULD NOT BE HERE \\
\hline & ENDIF & & \\
\hline $\mathbf{A D R}$ & CON & 0 & \\
\hline & $\begin{array}{l}\text { LIT } \\
\text { KMND }\end{array}$ & & \\
\hline
\end{tabular}




\begin{tabular}{|c|c|c|c|}
\hline & IF'EQ & $\bullet F, 2$ & ASSEMBLE IF FTN CALIED COMPASS \\
\hline & SA1 & A1 & ADR OF FIRST ARG TO X1 \\
\hline & SB1 & $\mathbf{X 1}$ & NOW TO B1 \\
\hline & SA1 & $A 1+1$ & STEP X1 TO NEXT ARG \\
\hline & SB2 & $\mathbf{X 1}$ & \\
\hline & SA1 & $\mathbf{A} 1+1$ & STEP X1 TO NEXT ARG \\
\hline & SB3 & $\mathbf{X 1}$ & \\
\hline & SA1 & $\mathbf{A 1 + 1}$ & STEP X1 TO NEXT ARG \\
\hline & SB4 & $\mathbf{X 1}$ & \\
\hline & SA1 & $\mathbf{A} 1+1$ & STEP X1 TO NEXT ARG \\
\hline & SB5 & $\mathbf{X} 1$ & \\
\hline & SA1 & $A 1+1$ & STEP X1 TO NEXT ARG \\
\hline & SB6 & $\mathbf{X 1}$ & \\
\hline & ENDIF & & \\
\hline & SAR & B5 & .GET SKIP \\
\hline & SB5 & $\mathbf{X} 2$ & SAVE SKIP \\
\hline & $\sqrt{P}$ & REG & \\
\hline GBYIE & CON & 0 & \\
\hline & IFEQ & $\bullet F, 2$ & ASSEMBLE IF FNTN CAILED COMPASS \\
\hline & SA1 & A1 & ADR OF FIRST ARG TO X1 \\
\hline & SB1 & $\mathbf{X} 1$ & NOW TO $\mathrm{B} 1$ \\
\hline & SA1 & $\mathbf{A 1 + 1}$ & STEP X1 TO NEXT ARG \\
\hline & SBR & $\mathbf{X 1}$ & \\
\hline & SA1 & $\mathbf{A 1 + 1}$ & STEP X1 TO NEXT ARG \\
\hline & SB9 & $\mathbf{X} 1$ & \\
\hline & SA1 & $\mathbf{A 1 + 1}$ & STEP X1 TO NEXT ARG \\
\hline & SB4 & $\mathbf{X} 1$ & \\
\hline & ENDIF & & \\
\hline & SB6 & $=1$ & SINGLE ITERATTON \\
\hline REG & SA1 & B1 & .POINT A1 AT START OF SOURCE STRING \\
\hline & SB7 & $\mathrm{B2}$ & .MOVE DESTTNATION ADDRESS \\
\hline & SA4 & $\mathrm{B3}$ & GET CALCUIATED OFFSET IN X4 \\
\hline & SA5 & B4 & GET BYTE SIZE IN X5 \\
\hline & NG & X5, GBERR & .NEGATTVE BITS IS ERROR \\
\hline & $\mathbf{5 x 3}$ & $\times 5-61$ & IF TRYING TO GET SUBSTRING GREATER \\
\hline & PL & X3, GBERR & .THAN 60 BITS,ERROR EXIT \\
\hline & SA3 & $\mathrm{BB}$ & \\
\hline & $\mathbf{Z R}$ & X3, GBYTE & .ZERO ITERATIONS MEANS NO ACTION \\
\hline & NG & X3,GBERR & NEGATTVE ITERATIONS IS ERROR \\
\hline & SB6 & $\mathbf{X 3 - 1}$ & .SET UP LOOP COUNTER \\
\hline & SBR & $\mathbf{X 5 - 1}$ & $. B 2=B Y T E$ SIZE-1 (SET FOR GENERATING MASK) \\
\hline LOOP & $\mathbf{S x 3}$ & 60 & CONSTANT OF 60 \\
\hline & PX7 & $\mathbf{X 3}$ & .FLOAT 60 \\
\hline & NX7 & $\mathbf{x 7}$ & .NORMAIIZE B \\
\hline & PX6 & $\mathrm{X} 4$ & .FLOAT NEW COFFSET (TN BITS) \\
\hline & NX6 & $\mathbf{X B}$ & .NORM \\
\hline & FX2 & $\mathbf{X B / X 7}$ & $. Q=A / B$ \\
\hline & UX2 & $\mathbf{B 1}, \mathbf{X 2}$ & . \\
\hline & LXR & $\mathbf{B 1}, \mathbf{X} 2$ & .GET INTEGER QUOTENT \\
\hline & $\overline{\mathbf{S B 1}}$ & $\mathrm{x} 2$ & .PUT IN B1 \\
\hline & PX2 & $\mathbf{X} 2$ & REPACK AND \\
\hline & NX2 & $\mathbf{X} 2$ & . \\
\hline & FX4 & $\times 7 \times 2$ & $. X 4=B * Q$ \\
\hline & FX4 & $\mathbf{X 6 - X 4}$ & $R=A-B * Q$ \\
\hline & UX4 & $\mathrm{B3}, \mathrm{X4}$ & .GET INTEGER \\
\hline & IX4 & $\mathrm{B3}, \mathrm{X} 4$ & . \\
\hline & $\mathbf{S A 1}$ & $\mathrm{A} 1+\mathrm{B} 1$ & GET NEW WORD IN \\
\hline & SAZ & $\mathbf{A 1 + 1}$ & .GET SECOND WORD (WHETHER NEEDED OR NNOT) \\
\hline & IX7 & $\mathbf{X} 3-\mathrm{X}_{4}$ & ACTUAL OFFSET = 60-CALC OFFSET \\
\hline & \$B3 & $\mathbf{X 7}$ & .PUT INTO BO \\
\hline & MXo & 1 & FORM THE \\
\hline & $\mathbf{A x O}$ & $\mathrm{B} 2, \mathrm{XO}$ & . \\
\hline & $\mathbf{I X 6}$ & X7-X5 & .FIND IF BYTE IS JUST IN FORD \\
\hline & SB1 & $\mathbf{X} \mathbf{8}$ & . \\
\hline & $\mathrm{IXO}$ & $B 9, \times 0$ & IINE UP MAXK \\
\hline
\end{tabular}


IBM 1130 VERSION

- W.HLTON - SCRIPPS INST.OF OCEANOG.-NORPAX PROJECT

- IEVEL 5 OF NORPAX BINARY DATA MGMT SCHEME

- JAN74 - CALIING SEQUENCES TAKEN FROM

- NATTONAL CENTER FOR ATMOSPHERIC RESEARCH TN/IA-93

- TECHNIQUES FOR THE PROCESSING, STORAGE AND

- EXCHANGE OF DATA

- BY ROY JENNE AND DENNIS JOSEPH.

- THESE ROUTINES ATTEMPT TO HELP TRANSIATTON

- BETWEen sone STRING OF BITS IN SOME aLIEN PATTERN

- AND aN ARRAY OF VALUES OR CHARACTERS IN THE NATTVE

- (IN THIS CASE, IBM 1600) INTERNAL FORMAT.

- Fortran calung SEQuences -

- CALL GBYTE (NPACK,ISAM,NSKIP,NBITS)

- CALL, GBYTES(NPACK,ISAM,NSKIP,NBITS,ISKIP, ITER)

- THERE -

- as a Result of aNy of the above calis, BIts are

- TRANSFERRED BETWEEN ARRAYS NPACK AND ISAM.

- NPACK IS THOUGHT OF AS ONE LONG STRING OF BITS.

- NPACK IS THOUGHT OF AS ONE IONG STRING OF BITS.

- ISAM IS THOUGHT OF AS GROUPS OF UP TO 16 BITS

- RIGHT-JUSTTFIED IN EACH ELEMENT.

- NSKIP=THE NUMBER OF BITS IN NPACK TO SKIP BEFORE

- ANY TRANSFER.

- NBITS=THE NUMBER OF BITS TO TRANSFER.

- GBYTE(IE. 'GET BIT STRING') DOES ONE 'TRANSFER FROM

- NPACK TO ISAM.

- ITER=THE NUMBER OF TRANSFERS TO BE MADE.

- ISKIP=THE NUMBER OF BITS IN NPACK TO SKIP BETWEEN

- TRANSFERS.

- NOTE THAT A TRANSFER TO NPACK WILL DISTURB ONLY THE

- EXACT NUMBER OF BITS TRANSFERRED, AND THE TRANSFER

- MAY STRADDLE TWO WORDS IN NPACK.

- howEVER, a TRANSFER To ISAM WILl DISTURB EXACTLY

- ONE WORD IN ISAM, NO MORE AND NO LESS, SINCE

- THE (16-NBITS) IEADING BITS WILL BE ZEROED, NO

- MORE THAN 16 BTS MAY BE TRANSFERRED, AND WORD

- boUNdaRIEs in ISAM WILl NOT BE STRADDLED.

- ERRORS IN THE CALITNG SEQUENCE WILL CAUSE a

- SYSTEM ERROR MESSAGE TO BE OUTPUT, AND THE JOB TO

- KIILEED. THE MESSAGE FORMAT IS...

- HEX WORD 2 ADDRESS OF CALL

- 3 NUNBER OF BITS REQUESTED FOR TRNSFR

- 4 ADDRESS OF PACKED ARRAY

- 5 adR OF UNPACKED ARRAY

- 6 NUMger OF TIERATIONS REQUESTED IF

- appllcable

- the remainder of the Message is as DOCUMENTEd IN

- 'ERROR MESSAGES AND RECOVERY PROCEDURES'

- NOTE THAT ITERATION COUNT MAY BE ZERO, IN WHICH

- CASE NOTHING IS DONE.SKIPS MAY BE NEGATTVE WHICH

- MAY CAUSE YOU TO MOVE THROUGH THE PACKED ARRAY IN

- ASSEM MLER SEQUENCE, BUT IF A BIT FETCH OR STORE

- STRADDLES A WORD BOUNDARY IN THE PACKED ARRAY, THE

- STRADDLF wILL BE HaNDLED IN A FORTRAN ARRAY SE-

- QUENCE, NOT ASSEMBLER SEQUENCE.

ENT GBYTE GET ONE BIT STRING

ENT GBYTES GET SEQUENCE OF BIT STRING

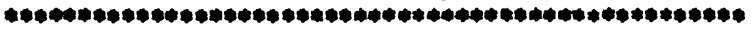

-

- 21 january 1981

- pRograM MODIFIED BY MadELEEINE zIRBES.

- ALL REFERENCES TO SBIT AND SBITS TERE REMOVED. 
ERMSG.FORTRAN

SUBROUTINE ERMSG(LEVEL,MSG,LUN,IARG)

C W. HIITON - NORPAX PROJECT - SCRIPPS INST. OF OCEANOG. - OCT74

C NORPAX DATA MANAGEMENT BINARY PACKED DATA CALUING SEQUENCE DEFINITTON

C GLOBAL LEVEL

C SEE NORPAX DATA MANAGEMENT TECH.MEMO NUMBER FOR EXTENSIVE

C DETAIS OF THE WHOLE SYSTEM.

C PRINTS ALL ERROR MESSAGES, DETERMINES WHETHER FATAL OR WARNING,ACTS

C ACCORDINGLY.

INTEGER LVDTA(4),EN,DE

C ** MACHINE DEPENDENT HOUSEKEEPING INFORMATTON **

C HOST MACHINE - CDC 7600

COMMON/DMGLBL/MACH,MXLUN,LUNCT,LISTP,IERRP,IUNS(5)

DATA EN,DE/RHEN,2HDE/

DATA LVDTA/6HPHYSCL, 6H BLOCK,6HREPORT,6H USER/ DATA LT,GT/2HLT,2HGT/

C COMPUTE CALL ADDRESS IF THAT IS GIVEN INSTEAD OF LEVEL ICALI=SHIFT(LEVEL, -30$)-400000004 \mathrm{~B}$

C ** END MACHINE DEPENDENT HOUSEKEEPING ***

ICHAR=I.EVEI 1

GO TO $(1000,2000,3000,4000,5000,6000,7000,8000,10000,9100,9200$,

$18000), M S G$

1000 WRITE(IERRP,1010)LVDTA(ICHAR),LUN

1010 FORMAT( 1XA6,* ACCESS ATTEMPTED ON NON-EXCSTENT LOGICAL UNIT *

1 IR)

CO TO 10000

2000 WRITE(IRRRP,2020) LVDTA(ICHAR),LUN, MXLUN

2080 FORMAT( 1XA6, * LOGICAL UNTT *I2, * OVERFLOWS TABLE CAPACTTY OF *,

1 IR, LOGICAL UNIIS.")

GO TO 10000

3000 WRITE(IERRP,3010)IARG,LVDTA(ICHAR),LUN,LT

3010 FORMAT(" SPECIFICATTON *,I2, * FOR *, A6, * LOGICAL UNIT *,I2,

1 - IS .,AR,*. THAN ALLOWED BY OTHER SPECS OR BY SYSTEM.*)

GO TO 10000

4000 WRITE(IRRRP,3010) IARG,LVDTA(ICHAR),LUN,GT

GO TO 10000

$5000 \quad$ IF (IISTP) 20000,20000,5005

6005 WRTYE(LISTP,5010) LVDTA(ICHAR),DE,LUN

5010 FORMAT( 1XA6,1XA2, *CODING ERROR ON LOGICAL UNIT *,IR)

CO TO 20000

6000 WRTIE(IERRP,5010)LVDTA(ICHAR), EN,LUN

GO TO 10000

7000 WRTTE(IERRP,7010)LVDTA(ICHAR),DE,LUN

7010 FORMAT( 1XA6, 1XA2, "CODE ARGUMENT ERROR ON LOGICAL UNIT *I2)

GO TO 10000

6000 WRITE(IRRRP, 7010)LVDTA(ICHAR),EN,LUN

GO TO 10000

8100 WRITE(IIRRRP,9110)LUN

9110 FORMAT(* BLOCK INPUT CHECKSUM ERROR ON UNIT *I2)

GO TO 10000

8200 PRINT 9210,ICALL, LUN, IARG

Q210 FORMAT(* IIIEGAL CALL, TO GBYTE(S) FROM *,07,* MOVING DATA FROM STRI

1 NG BEGINNING *,020,* TO ARRAY TORD AT *,07,**)

GO TO 10000

9300 PRINT 9310,ICAII, LUN,IARG

9310 FORMAT( * IIJEGAL CALL TO SBIT(S) FROM *,07, MOVING DATA TO STRING

C10000 STOP

1 AT *,07,* FROM ARRAY WORD CONTAINING *,020,**)

10000 RETURN

20000 RETURN

ENND 
- SBIT (STORE BIT STRING) WAS THE EXACT INVERSE

- OF GBYTE.

-

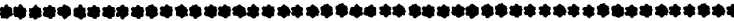

- MACHINES:

- IBM 1130

IBM 1800

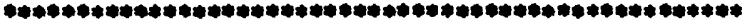

- BEgIN MACRO DEFINITIONS FOR GBYTES,ETC.

$$
\begin{array}{ll}
\text { MAC } & \\
\text { STUP } & \text { ENTRY,SAVE,RTRN,NARGS, UNP } \\
\text { ILST } & \text { OFF }
\end{array}
$$

- SAVE REGS,GET ARGS, CHECK BIT CNT,SET UP RET

STX $1 \mathrm{SAVE}+1$

STX \& SAVE+3

IDX I1 ENTRY

ID 10

STO ENTRY

ID 11

STO UNP+1

ID II 3

STO NBITS

BN ERR

AIF (NARGS IEE 5),IFEND

ID II 4

STO ISKIP

STX 3 SAVE+5

ID I1 5

STO \&1

IDX IB *

BN ERR

IFEND ANOP

$$
\text { ID }=16
$$

S NBIIS

STO NB16

BN IRRR

NDX 1 NARGS

STX 1 RTRN + 1

AIF (NAFGS LE 5),IFEND

IDX 90

$B \quad+1$

B SAVE

IFEND ANOP

IIST ON

MFND

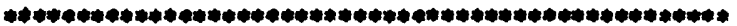

MAC

CPDSP ENTRY

IIST OFF

- COMPUTE DISPLACENENT IN PACKED ARRAY

SRT 4

RTE 16

SRA 12

STD SKIP

ID ENTRY

$\$$ DISP

STO ENTRY

IDX IR ENTRY

IIST ON

MTID

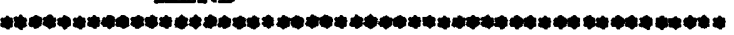

MAC

GBYT
SAVE REG ONE

SAVE REG TWO

PTR TO ARG ADRS TO REG ONE

GET ADR OF PACKED ARRAY

USE ENTRY AS STORAGE

GET ADR OF DESTINATION WORD

GET NUMBER OF BITS

AND SAVE TT.

BR IF NO.OF BITS NEG,ERROR

GET SKIP TO USE IN IOOP

AND SAVE TT.

SAVE REG 3

GET ITERATION COUNT

PUT IN REG 3 LOAD

ITER TO REG 3 AS LOOP CTR

BR IF ITERATION NEG,ERROR

VALUE 16 TO A-REG

SUBTRACT NUMBER OF BITS

SAVE 16-NBITS

EXRR IF .GT.16

POINT REG ONE AT RETURN

TEST ITERATION COUNT FOR ZERO

CONTINUE IF POSITIVE

RETURN IF ZERO

DIVIDE BY 16

SWAP DISP AND SKIP

RIGHT JUSTTF'Y SKIP

SAVE SKIP AND DISP

GET ADR OF PACKED ARRAY

SUBIRACT DISPLACEMENT

PTR TO PACKEDD ARRAY TO R2 
- MACRO TO GET ONE BIT STRING

\begin{tabular}{|c|c|c|c|}
\hline & LIST & OFF & \multirow[b]{2}{*}{ SKIP IN REG ONE } \\
\hline & LDX & I1 SKIP & \\
\hline & D & $2-1$ & GET SECOND PACKED WORD \\
\hline & SRT & 16 & IN Q REG. \\
\hline & L D & 20 & GET FIRST PACKED WORD. \\
\hline & SLT & 1 & LEFT SHIFT BY SKIP \\
\hline SRAB & DC & 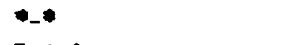 & WILL RT SHFT BY 16-NBITS \\
\hline \multirow[t]{2}{*}{ STUNP } & $\begin{array}{l}\text { STO } \\
\text { LTST }\end{array}$ & $\begin{array}{l}\mathrm{L} * * \\
\mathrm{ON}\end{array}$ & RETURN RESULT \\
\hline & \multicolumn{3}{|c|}{$\begin{array}{l}\text { WST } \\
\text { MEND }\end{array}$} \\
\hline & \multicolumn{3}{|c|}{ MAC } \\
\hline & SBYT & \multirow{2}{*}{\multicolumn{2}{|c|}{$\begin{array}{l}\text { SSIA,SSRA, GTUNP } \\
\text { OFF }\end{array}$}} \\
\hline & LIST & & \\
\hline \multicolumn{4}{|c|}{ - MACRO TO STORE ONE BIT STRING } \\
\hline & SLT & 16 & CLEAR Q-REG \\
\hline \multirow{6}{*}{$\begin{array}{l}\text { GTUNP } \\
\text { SSILA }\end{array}$} & L D & L*_* & GET UNPACKED WORD \\
\hline & $\mathrm{DC}$ & $*$ & WILL SHFT LEFT 16-NBITS \\
\hline & IDX & I1 SKIP & SKIP TO REG ONE \\
\hline & RTE & 1 & SHIFT BY SKIP \\
\hline & STD & NUBTS & SAVE TWO WORDS,MAY STRADDLE \\
\hline & LDD & ONES & GET 32 ONES \\
\hline \multirow[t]{10}{*}{ SSRA } & DC & $*$ & WIL SHFT,GEN NBITS ZEROES \\
\hline & RTE & 1 & MOVE THEM OVER BY SKIP \\
\hline & AND & 20 & AND SAVE PACKED DATA, OR IN \\
\hline & $\mathbf{O R}$ & NUBTS & NEW DATA. \\
\hline & STO & 20 & RETURN FIRST WORD OF RSLT \\
\hline & SLT & 16 & RETRIEVE REST OF MASK FM Q \\
\hline & AND & $2-1$ & AND REPEAT FOR SECOND WORD \\
\hline & OR & NUBTS+1 & OF RESULT. \\
\hline & STO & $2-1$ & RETURN POSSIBIE OTHER WORD \\
\hline & LIST & ON & \\
\hline
\end{tabular}

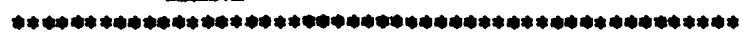

- END MACRO DEFINITONS,BEGIN EXECUTABIE CODE

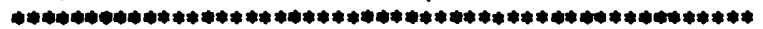

- HERE BEGINS ACTUAL BODY OF SUBROUTINE GBYTE

\begin{tabular}{|c|c|c|}
\hline $\begin{array}{l}\text { GBYTE } \\
\text { GETYB }\end{array}$ & $\begin{array}{l}\text { EQU } \\
\text { DC }\end{array}$ & 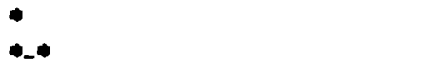 \\
\hline & STUP & GETYB,GSAVE,GRET,4,GUNP \\
\hline & ID & NB16 \\
\hline & OR & SRA \\
\hline & STO & GSRA \\
\hline & LD & II - 2 \\
\hline & CPDSP & GETYB \\
\hline & GBYT & GSRA,GUNP \\
\hline GSAVE & LDX & L1 \\
\hline & IDX & L2* * \\
\hline GRET & $\mathbf{B}$ & $L \bullet-$ \\
\hline - onementerer & $0+* * * * * \infty$ & 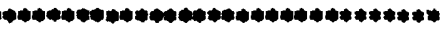 \\
\hline GBYTES & EQU & $\bullet$ \\
\hline GBYIS & $\mathrm{DC}$ & $\bullet$ \\
\hline & $\begin{array}{l}\text { STUP } \\
\text { ID }\end{array}$ & $\begin{array}{l}\text { GBYIS,GSAVS,GRETS,6,GUNPS } \\
\text { NB16 }\end{array}$ \\
\hline & OR & SRA \\
\hline & \$TO & GSRAS \\
\hline & DE & I1 -4 \\
\hline GLOOP & EQU & - \\
\hline & CPDSP & GBYTS \\
\hline & GBYT & GSRAS,GUNPS \\
\hline & $\mathrm{LD}$ & ISKTP \\
\hline & $\mathbf{A}$ & 8KIP \\
\hline & A & NBITS \\
\hline & MDX & L GUNPS+1,-1 \\
\hline
\end{tabular}

GET ONE BIT STRING

GET 16-NBITS,MAKE UP SHIFT INSTRUCTION

GET SKIP

GET SEQUENCE OF BITS

GET 16-NBITS,MAKE UP SHIFT

INSTRUCTION

GET SKIP

TOP OF LOOP

STEP AHEAD ISKTP BITS

PLUS NUMBER OF BITS

STEP THRU UNPACKED ARRAY 


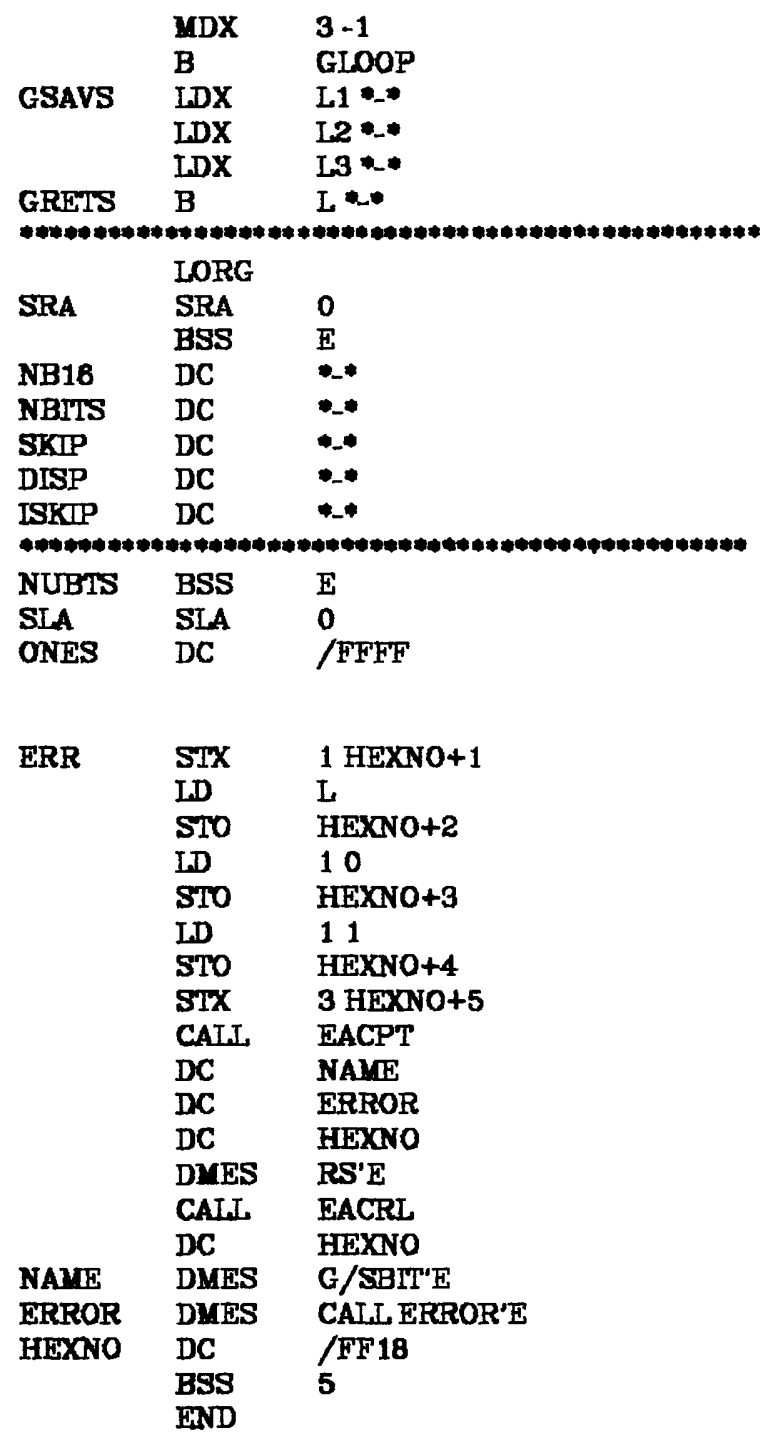

DECR,TEST REG 3

RESTORE REG ONE

RESTORE REG TWO

RESTORE REG THREE RETURN

TEMPIATE INSTRUCTION

16-NBITS,EVEN ADDRESS

SLOT FOR BIT COUNT

SLOT FOR SKIP WTTHIN LOOP

2

TEMPIATE INSTRUCTION

AT ODD ADDRESS

PUT CALI ADDRESS IN MSG

NBITS

GET PACKED ARR ADR

GET UNPACKED ARR ADR

STORE ITERATION COUNT IF ANY

CAUSE RESTART

POINT TO LEVEL/AREA

ASSUME VCORE/BASIC IEVEL 


\section{PDP11 VERSION}

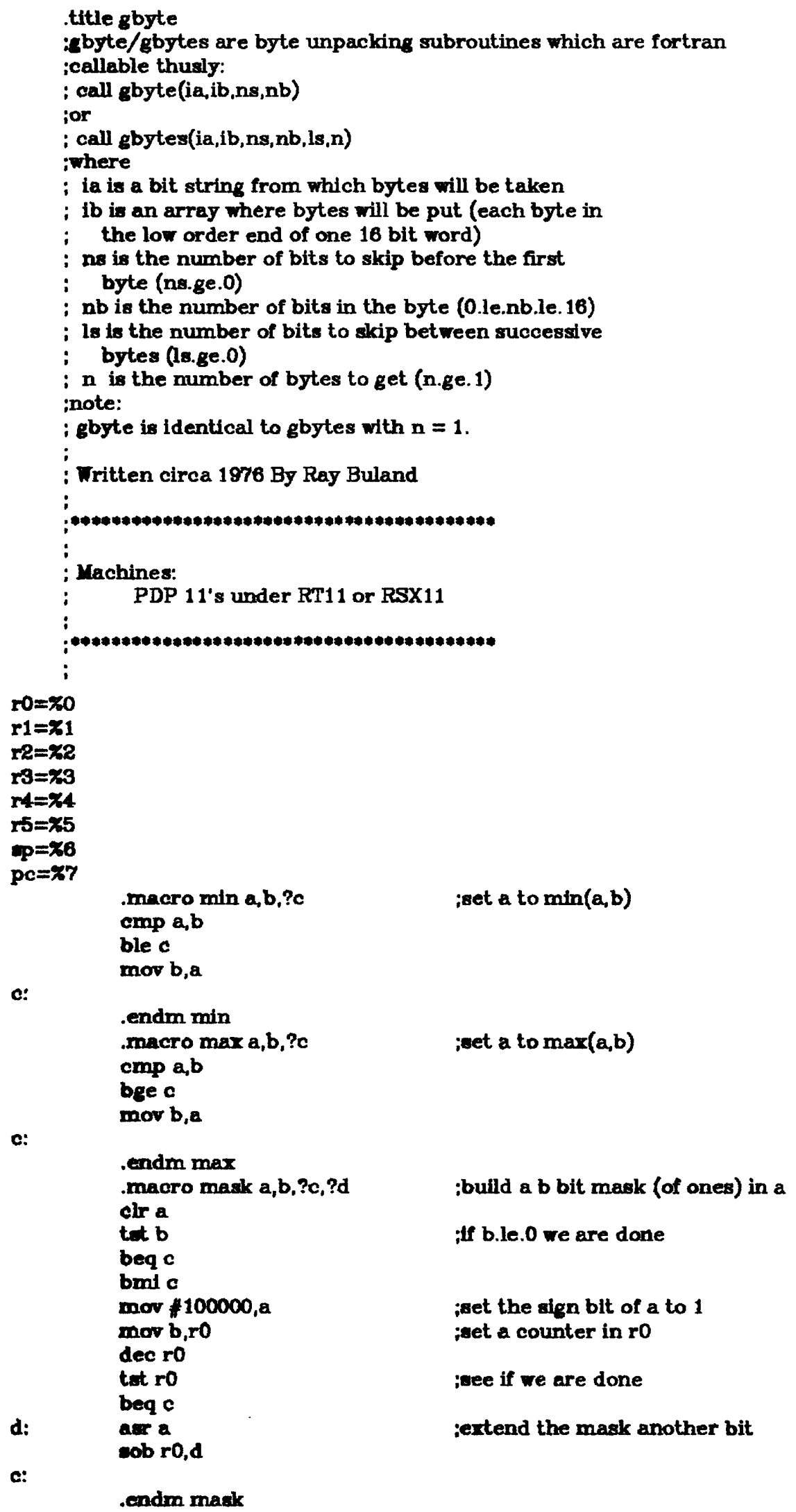




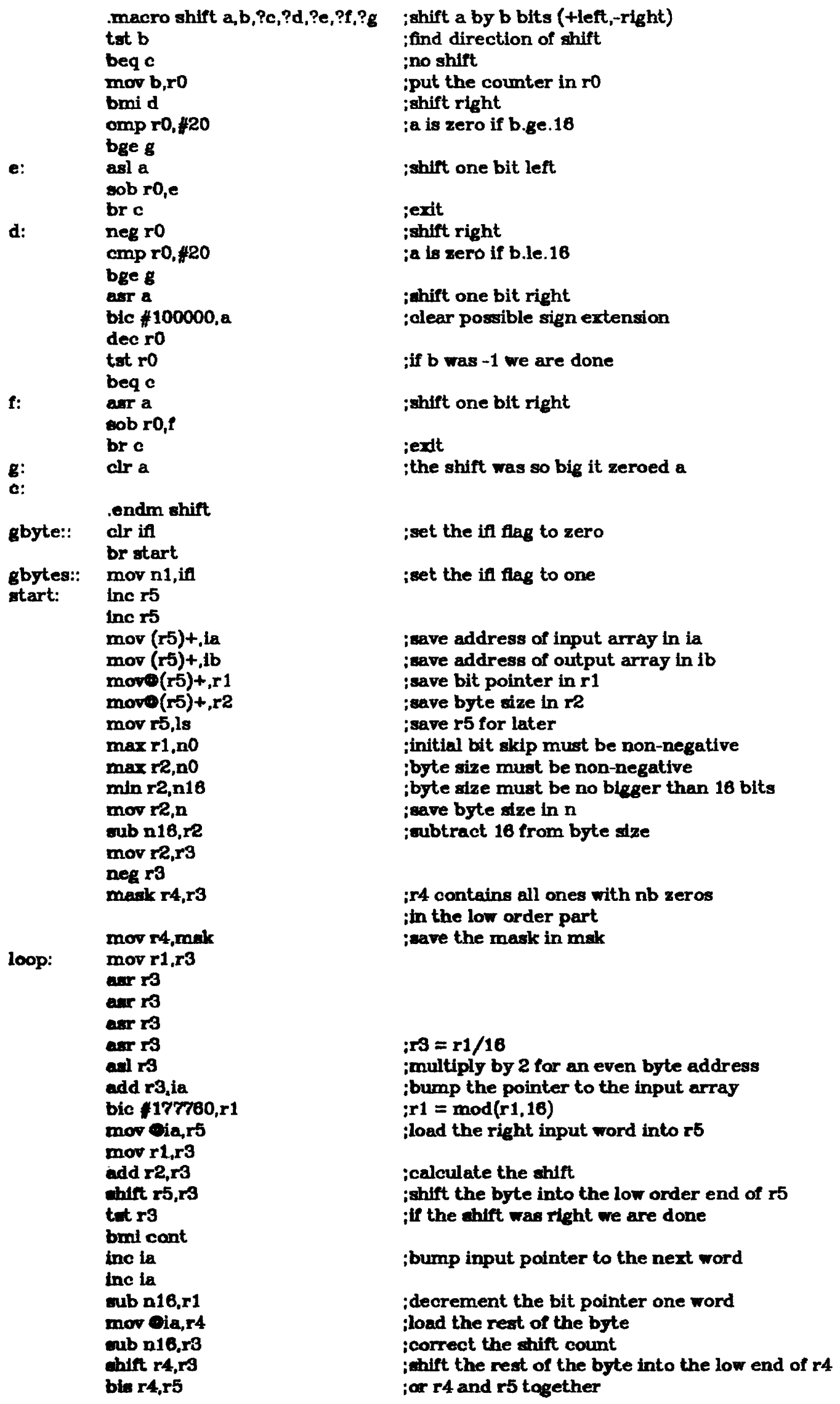




\begin{tabular}{|c|c|}
\hline $\begin{array}{l}\text { out: } \\
\text { la: } \\
\text { ib: } \\
\text { ls: } \\
\text { n: } \\
\text { msk: } \\
\text { if: } \\
\text { no: } \\
\text { n1: } \\
\text { n16: }\end{array}$ & 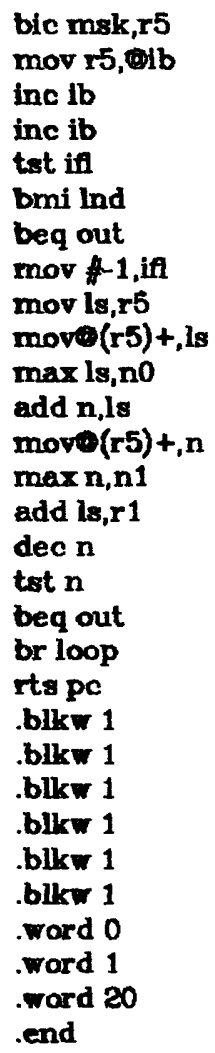 \\
\hline
\end{tabular}

;and out the high order part of $r 5$

;move 5 into the output array

;bump the output pointer to the next word

if if is zero we are finished

:go around again

igbyte exit

;set if to -1

;reset r5 to pick up the other arguments of gbytes ;move secondary bit skip to ls

is must be non-negative

;add in the byte size

;move the number of bytes to $n$

in must be at least one

:bump bit pointer to next byte

;if $\mathrm{n}$ is still positive go around again

;return 


$$
\text { C }
$$

SUBROUTINE GBYTES(IA,IB,NS,NB,IS,N)

C GBYTES IS A BYTE UNPACKING SUBROUTINE.

C

C THE SYNTAX IS:

C

C CALL GBYTES (IA, IB, NS, NB, IS, N)

c

C

c

c IA IS THE BIT STRING FROM WHICH THE

C BYTES WILL B TAKEN,

c

c IB IS AN ARRAY WHERE THE BYTES WTLL

C BE PUT,

C

C NS IS THE NUMBER OF BITS TO SKIP

C BEFORE THE FIRST BYTE,

C

C NB IS THE NUMBER OF BITS IN THE BYTE,

C

C NS IS THE NUMBER OF BITS TO SKIP

C BETWEEN SUCCESSIVE BYTES, AND

C

C N IS THE NUMBER OF BYTES TO EXTRACT.

c

C

C CALIS GBYTE

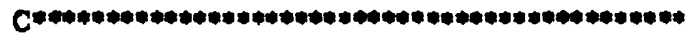

\section{MACHINES:}

C $\operatorname{VAX} 11 / 780$

C VAX 11/750 UNDER VMS

C ASSUMING INTEGERS ARE INTEGER*4

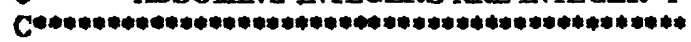

C

C PROGRAMNET SEPTEMMER 17, 1980 BY RAY BULAND

C

C

DIMENSION $\operatorname{IA}(1), \mathbb{B B}(1)$

$\mathbf{K B}=\mathrm{NB}+\mathrm{IS}$

$\mathrm{KS}=\mathrm{NS}$

DO $1 \mathrm{I}=1, \mathrm{~N}$

CALL GBYTE(IA,IB(I),KS,NB)

$1 \mathrm{KS}=\mathrm{KS}+\mathrm{KB}$

RETURN

EHND

C

SUBROUTINE GBYTE(IA,IB,NS,NB)

C GBYTE IS A BYTE UNPACKING SUBROUTINE.

C

C THE SYNTAX IS:

C

C CALL GBYTE (IA, IB, NS, NB)

C

C WHERE AIL PARAMETERS ARE AS DEFTNED

C IN GBYTESS. IN THIS CASE, $\mathrm{N}=1$.

C

C

DIMENSION IA(1)

IF(NB.LE.0) GO TO 13 
NSIZ $=$ MINO(NB, 32)

NBIT $=$ MAXO(NS, O) +NSIZ

$N W D=(N B I T+31) / 32$

IB =IJB\$EXTZV (32*NWD-NBIT,NSIZ,IA(NWD))

RETURN

13 IB $=0$

RETURN

END 
C VERSTON

/ Gbytes- treats ia as a bit string.

$n$ bytes each ibits long are extracted from ia and placed in the low arder end of successive wards of ib (zero padded to the left).

is bits are skipped before the first byte.

ns bits are akipped between successive bytes.

SYNTAX:

C:

int $\mathrm{ia}[10], \mathrm{ib}[10]$;

int is, ibits, ns, $n$;

gbytes- (ia, ib, Lis, \&ibits, \&ms, \&n);

Fortran:

integer $\mathrm{la}(10), \mathrm{ib}(10)$

integer is, ibits, ns, $n$

call gbytes (ia, ib, is, ibits, ns, n)

Calls getbits

MACHINES:

Unix

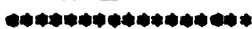

\section{Programmed 15 September 1980} by Madeleine Zirbes

$\%$ gbytes-(ia, ib, is, ibits, ns, n)

int Ha;

int $1 \mathrm{~b}$;

int

int Nbits;

int tns;

int "n;

1

int i;

int bit, word, bits;

bits = is;

for $(1=0 ; 1<m ; 1+t)$ |

bit = bitaxis:

word = (bits - bit) $/ 16$;

bit $=15$ - bit;

ib+t = getbita((unsigned)(ia[word]).

(undened)(bit).

(undened)(-ibits))

bits = bits + थbits + *ns;

)

3

retbite(x, p, n)

\% Getbits gets $n$ bits from position p.

From:

The C Programming Language

Brian N. Kernighan

Dennis M. Ritchie

Bell Laboratories 
PL1 VERSION

gbytes: proc (in, out, ns, nb, is, n);

$1 *$

GBYTES

Gbytes is a byte unpacking subroutine.

The ayntax is:

declare (in, out, ns, nb, is, n) fixed bin (35);

call gbytes (in, out, ns, nb, is, n);

where:

In is the bit string from which bytes will be taken,

out is the output array where bytes wlll be placed.

ns is the number of bits to skip before the first byte,

$\mathrm{nb}$ is the number of bits to retrieve.

is is the number of bits to be skipped between succesdive bytes, and

$n$ is the number of bytes to extract from in.

Calls no other routine.

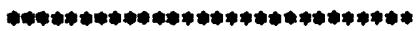

MACHINES:

Honeywell Multics

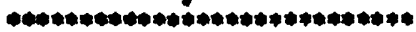

Programmed August, 1978 by Ray Buland

$\%$

del (in, out, $n s, n b, i s, n$ ) fired bin (35);

del 01 ia based (addr (in)) unaligned,

02 fikip bit (ns).

$02 \mathrm{na}(\mathrm{n})$,

09 byte bit (nb).

09 alrip bit (is):

del 01 ib $(n)$ based (addr (out)) unaligned,

02 zero bit (36-nb).

OR byte bit (nb):

1b.zero = 'm'b;

lb.byte = na.byte;

return:

end; 
Murray Hill, New Jersey

Prentice-Hall

Calls no other routine.

$\because$

unsigned $\mathbf{x}, \mathbf{p}, \mathrm{n}$;

I

return((x>>(p+1-n))\& $\sim(\sim 0<<n))$;

1" Note:

"Declaring the argument $\mathrm{x}$ to be unsigned ensures that when it is right-shifted. vacated bits will be miled with zeroes, not sign bits, regardless of the machine

$\%$

the program is run on."

3 


\section{Network-Day Tape Software Users Guide}

Report Form

Please indicate any errors, suggested additions or deletions, or general comments below.

Mail to:

Madeleine Zirbes

U.S. Geological Survey

MS 967 Box 25046

Denver Federal Center

Denver, CO 80225 